Universidad deValladolid

PROGRAMA DE DOCTORADO EN INGENIERÍA TERMODINÁMICA DE FLUIDOS

TESIS DOCTORAL:

\title{
DETERMINACIÓN DE LA ENVOLVENTE DE FASES DE MEZCLAS BINARIAS DE COMPONENTES DEL BIOGÁS CON UN RESONADOR DE MICROONDAS
}

Presentada por Rodrigo Susial Martín para optar al grado de Doctor por la Universidad de Valladolid

\author{
Dirigida por \\ Dr. José Juan Segovia Puras \\ Dra. María del Carmen Martín Gonzalez
}

Valladolid, 2019 

"One of the principal objects of the theoretical research is to find the point of view from which the subject appears in the greatest simplicity"

Josiah Willard Gibbs (Rumford Medal 1881) 



\section{Agradecimientos:}

Al Dr. José Juan Segovia Puras y a la Dra. Mạ Carmen Martín, mi más sincero agradecimiento por haber estado bajo su dirección en el desarrollo de esta tesis doctoral.

A los profesores, Dr. Miguel Ángel Villamañán, Dr. César Chamorro y Dra. Rosa Villamañán gracias por vuestra franqueza, consejos y ayuda en los momentos que más se necesitaban. Gracias Eduardo Concepción, y Jorge Rubio, compañeros de máster y desde el principio de este viaje. A los compañeros de laboratorio: Dr. Ángel Gómez, Laura de Pablo, Daniel Lozano, Roberto Hernández y Eduardo Pérez por vuestro apoyo.

Al Centro Español de Metrología (CEM), a través de la Dra. Mạ Dolores del Campo, la cual nos ha proporcionado de los equipos y mezclas necesarias para llevar a cabo esta tesis.

A mi familia, a todas y a todos les dedico esta tesis doctoral. 

A la Junta de Castilla y León por las ayudas destinadas a financiar la contratación predoctoral de personal investigador, cofinanciadas por el Fondo Social Europeo, publicada en la ORDEN EDU/310/2015, de 10 de abril.

A la Junta de Castilla y León por el programa de apoyo a proyectos de investigación cofinanciadas por el Fondo Europeo de Desarrollo Regional publicada en la ORDEN EDU/756/2016, de 26 de agosto, bajo el título: “Revalorización de recurso renovables regionales biomásicos y eólicos para la producción de gases energéticos sostenibles (biogás e hidrógeno) y su incorporación en la red de gas natural".

A la Junta de Castilla y León por el programa de apoyo a proyectos de investigación cofinanciadas por el Fondo Europeo de Desarrollo Regional publicada en la ORDEN EDU/1255/2018, de 20 de noviembre, bajo el título: “Caracterización de gases energéticos formulados a partir de biogás e hidrógeno-eólico para la mejora de sus procesos de obtención, depuración y uso, dentro de la estrategia regional de desarrollo de la bioeconomía. Acrónimo (bioecogás)".

Al Ministerio de Economía y Competitividad de España, bajo el título:

“Gases energéticos: biogás y gas natural enriquecido por hidrógeno (ENE201347812-R)".

Al Ministerio de Economía y Competitividad de España, bajo el título:

“Caracterización de gases energéticos sostenibles (biogás e hidrógeno), producidos con recursos renovables biomásicos y eólicos, para su incorporación a la red de gas natural (ENE2017-88474-R)". 



\section{Resumen}

El trabajo de investigación desarrollado en esta Tesis Doctoral ha sido el diseño y puesta en marcha de una nueva cavidad cilíndrica resonante en microondas con un tubo de zafiro situado en el eje central de la cavidad que contendrá la muestra a analizar, para la determinación del equilibrio de fases a alta presión de sustancias presentes en el biogás y el gas natural.

La validación del equipo se ha llevado a cabo mediante la determinación de la curva de vaporización del $\mathrm{CO}_{2}$, comparando los resultados obtenidos mediante la ecuación de estado de Span \& Wagner. Además, se han medido la envolvente de fases de las mezclas binarias formadas por $\mathrm{CO}_{2}+\mathrm{CH}_{4} ; \mathrm{CO}_{2}+\mathrm{C}_{3} \mathrm{H}_{8}$ y $\mathrm{CO}_{2}+\mathrm{N}_{2}$ a diferentes composiciones en $\mathrm{CO}_{2}$.

Estas mezclas además de ser comparadas con la literatura existente, se han estudiado mediante la ecuación de estado GERG-2008, comparando las desviaciones obtenidas experimentalmente con los devueltos por medio de esta ecuación. Además, se ha empleado la ecuación de estado de Peng-Robinson, por medio de las reglas de mezclas de Wong \& Sandler con el propósito de obtener los coeficientes de ajuste de dicha ecuación y evaluar el ajuste obtenido con las mezclas estudiadas. 



\section{Abstract}

The research work developed in this Doctoral Thesis has been the design and implementation of a new microwave resonant cavity with a sapphire tube located in the central axis of the cavity that will contain the sample to be analyzed, for the determination of the equilibrium of phases at high pressure of substances present in biogas and natural gas.

The validation of the apparatus was carried out by determining the $\mathrm{CO}_{2}$ vaporization curve, comparing the results obtained with Span \& Wagner equation of state. In addition, the phase envelope of the binary mixtures formed by $\mathrm{CO}_{2}+\mathrm{CH}_{4} ; \mathrm{CO}_{2}+\mathrm{C}_{3} \mathrm{H}_{8}$ and $\mathrm{CO}_{2}+\mathrm{N}_{2}$ was been measured at different compositions in $\mathrm{CO}_{2}$.

These mixtures, besides being compared with the existing literature, have been studied using the GERG-2008 equation of state, comparing the deviations obtained experimentally with those returned by means of this equation. In addition, the Peng-Robinson equation of state has been used, by means of the Wong \& Sandler mixing rules with the purpose of obtaining the adjustment coefficients of this equation and evaluating the adjustment obtained with the studied mixtures. 



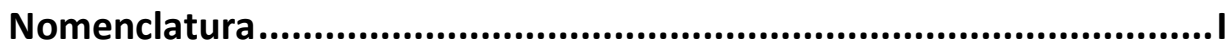

Símbolos I

Símbolos griegos III

Subíndices IV

Superíndices IV

Abreviaturas IV

Compuestos químicos $\quad \mathrm{V}$

Índice de Tablas ...................................................................................VII

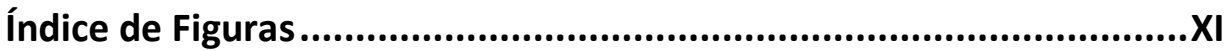

CAPITULO 1 INTRODUCIÓN .......................................................... 17

1.1 El problema medioambiental 1

1.2 Antecedentes 4

1.3 Objetivos 5

$\begin{array}{lll}1.4 & \text { Estructura de la tesis } & 7\end{array}$

\section{CAPITULO 2 PROPIEDADES TERMODINÁMICAS Y ELECTROMAGNÉTICAS}

DE LOS FLUIDOS

2.1 Introducción 11

$\begin{array}{lll}2.2 & \text { Propiedades del equilibrio } & 12\end{array}$

2.2.1 El potencial químico......................................................... 12

2.2.2 La fugacidad y el coeficiente de fugacidad ........................... 14

2.2.3 Regla entre fases ......................................................... 16

2.2.4 Equilibrio de fases a alta presión ........................................... 17

2.2.5 Diagrama de fases a alta presión ......................................... 18

2.3 Propiedades electromagnéticas de los fluidos 20

2.3.1 Concepto de constante dieléctrica ...................................... 21 
2.3.2 Concepto de red de microondas ............................................. 26

2.4 Relación entre propiedades volumétricas y dieléctricas 29

\section{CAPITULO 3 TÉCNICAS EXPERIMENTALES PARA LA MEDIDA} DETERMINACIÓN DEL EQUILIBRIO A ALTA PRESIÓN .............................. 33

3.1 Introducción 35

3.2 Técnicas experimentales para la medida del equilibrio a alta presión

3.3 Técnicas experimentales para la medida de propiedades dieléctricas

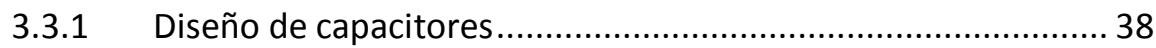

3.3.2 Cavidad cilíndrica resonante con guía evanescente.................. 41

3.3.3 Cavidad reentrante resonante ................................................ 42

3.3.4 Cavidad cuasi esférica resonante............................................. 44

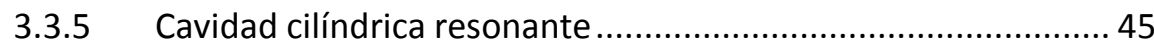

3.4 Cavidad cilíndrica resonante en microondas empleada en esta tesis doctoral 48

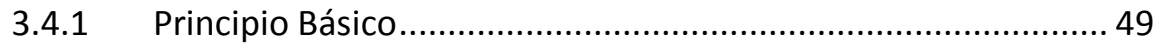

3.4.2 Cavidad cilíndrica con tubo de muestreo.................................. 56

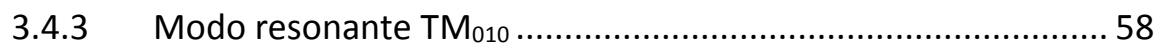

3.4.4 Medida del factor de calidad y frecuencia de resonancia .......... 61

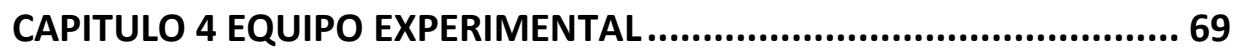

4.1 Introducción $\quad 71$

4.2 Descripción de la cavidad cilíndrica resonante en microondas 71

4.3 El sistema experimental 77

4.3.1 El analizador vectorial de redes (VNA) ................................... 79

4.3.2 Instrumentos para la medida de la temperatura ....................... 80

4.3.3 Instrumentos para la medida de la presión ................................ 80 


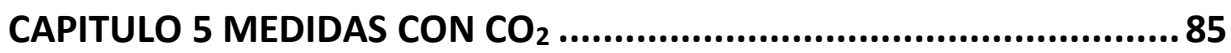

5.1 Introducción 87

5.2 Procedimiento experimental 87

5.3 Determinación de las incertidumbres 89

5.4 Resultados experimentales 92

5.5 Análisis de los resultados 103

\section{CAPÍTULO 6 ENVOLVENTE DE FASES Y MODELIZACIÓN DE SITEMAS}

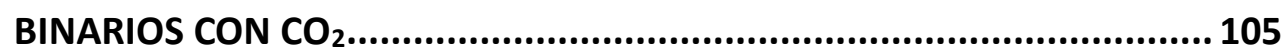

6.1 Introducción 107

6.2 Preparación gravimétrica de las muestras gaseosas 108

6.3 Determinación experimental de la envolvente de fases 109

6.4 Determinación de las incertidumbres 110

6.5 Modelización de los datos experimentales 111

6.6 Resultados experimentales 118

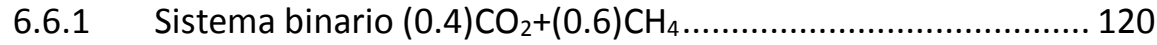

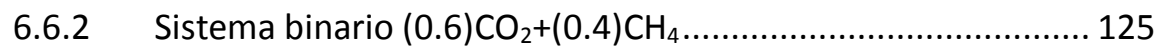

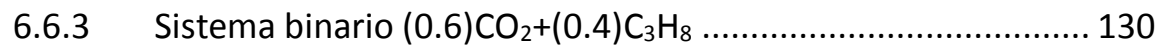

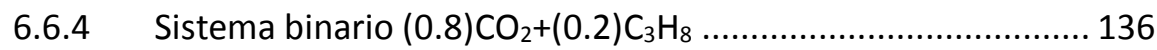

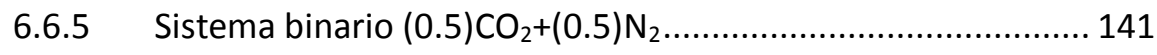

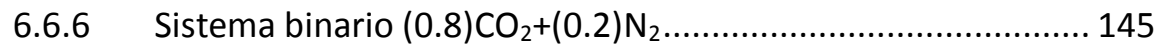

6.7 Análisis de los resultados 149

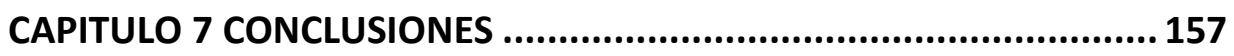

$\begin{array}{lll}7.1 & \text { Introducción } & 159\end{array}$

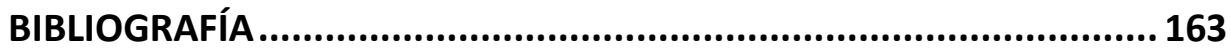




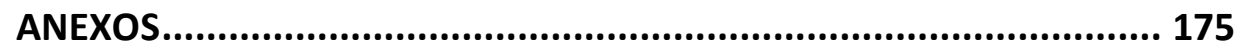

Anexo l: Diagramas y planos de la celda de medida

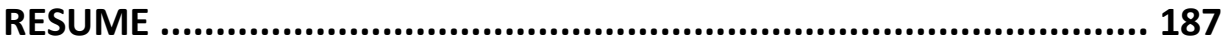




\section{Nomenclatura}

\section{Símbolos}

$A_{\varepsilon} \quad$ Primer coeficiente virial dieléctrico

$\mathrm{A}_{\mu} \quad$ Termino dipolar ecuación Harvey y Lemmon

A Constante método perturbación/Parámetro modelado resonancia/Amperio/Energía libre de Helmholtz

$A^{E} \quad$ Energía de exceso de Helmholtz

a Radio cavidad cilíndrica

Parámetros ecuación Harvey y Lemmon/Parámetros ajuste polinomio fase homogénea

B

Vector de densidad de flujo magnético/Constante método perturbación/Parámetro modelo resonancia

$\mathrm{B}_{\varepsilon} \quad$ Segundo coeficiente virial dieléctrico

$\overline{\mathrm{B}} \quad$ Amplitud compleja

$b_{0}, b_{1} \quad$ Parámetros ecuación Harvey y Lemmon

Capacidad eléctrica/Parámetro modelado

C resonancia/Parámetro ecuación Harvey y Lemmon/parámetro método perturbación

$\mathrm{C}_{\varepsilon} \quad$ Tercer coeficiente virial dieléctrico

c Velocidad de la luz

$\mathrm{C}_{0}, \mathrm{C}_{1} \quad$ Parámetros ecuación Harvey y Lemmon

D Vector de desplazamiento eléctrico/Parámetro modelado resonancia/ parámetro ecuación Harvey y Lemmon

d Diámetro exterior del tubo

E

Vector de intensidad de campo eléctrico/Parámetro modelado resonancia

e Espesor tubo

F Número de grados de libertad

f $\quad$ Fugacidad/Frecuencia 


\begin{tabular}{|c|c|}
\hline G & Conductancia eléctrica/Energía libre de Gibbs \\
\hline $\mathrm{G}^{\mathrm{E}}$ & Energía de exceso de Gibbs \\
\hline $\mathrm{H}$ & Vector de intensidad de campo magnético \\
\hline 1 & Intensidad eléctrica \\
\hline $\mathrm{I}_{1}, \mathrm{I}_{2}$ & Intensidad de corriente puertos 1 y 2 \\
\hline J & Vector de densidad de corriente eléctrica \\
\hline $\mathrm{j}_{\mathrm{n}}^{\prime}$ & Función de Besel de primer orden \\
\hline $\mathrm{k}$ & Coeficiente de cobertura/Constante dieléctrica \\
\hline $\mathrm{k}_{\mathrm{B}}$ & Cte. De Boltzmann $\left(1.346 \times 10^{-23} \mathrm{~J} \mathrm{~K}^{-1}\right)$ \\
\hline L & Inductancia \\
\hline I & Longitud cavidad cilíndrica \\
\hline M & Peso molecular \\
\hline $\mathrm{m}$ & Número de componentes de una mezcla \\
\hline $\mathrm{N}$ & Newton \\
\hline $\mathrm{N}_{\mathrm{A}}$ & Número de Avogadro $\left(6.022 \times 10^{23}\right)$ \\
\hline $\mathrm{n}$ & Número de moles /Índice de refracción \\
\hline$P$ & Potencia \\
\hline $\mathrm{P}_{\mathrm{m}}$ & Polarización molar \\
\hline $\mathrm{p}$ & Presión/Condición de contorno/Parámetro suavizado "spline" \\
\hline Q & Factor de calidad \\
\hline $\mathrm{R}$ & $\begin{array}{l}\text { Cte. universales de gases }\left(8.314 \mathrm{~m}^{3} \mathrm{~Pa} \mathrm{~K}^{-1} \mathrm{~mol}^{-1}\right) / \text { Resistencia } \\
\text { eléctrica }\end{array}$ \\
\hline r & Coordenada cilíndrica/Coordenada polar \\
\hline $\mathrm{s}$ & Entropía/Parámetro de dispersión \\
\hline $\mathrm{s}$ & Módulo de tracción/Función polinómica trazadora \\
\hline
\end{tabular}




$\begin{array}{cl}\text { T } & \text { Temperatura/Pérdidas por transmisión } \\ \mathrm{t} & \text { Tiempo/Coordenada polar } \\ \mathrm{U} & \text { Potencial eléctrico/Incertidumbre expandida } \\ \mathrm{u} & \text { Incertidumbre estándar asignada } \\ \mathrm{V} & \text { Volumen } \\ \mathrm{v} & \text { Cambio de variable } \\ \overline{\mathrm{X}} & \text { Composición molar } \\ \mathrm{x}_{\mathrm{i}, \mathrm{y}_{\mathrm{i}}} & \text { Composiciones molares sustancia i } \\ \mathrm{W}_{\mathrm{i}} & \text { Peso punto i curva "spline" } \\ \mathrm{z} & \text { Coordenada cilíndrica } \\ \mathrm{Z}_{0} & \text { Impedancia característica }\end{array}$

\section{Símbolos griegos}

$\begin{array}{ll}\nabla & \text { Operador nabla de Hamilton } \\ \cos \theta_{\mathrm{e}} & \text { Factor de potencia dieléctrico } \\ \tan \delta_{\mathrm{e}} & \text { Tangente de pérdidas dieléctricas } \\ \alpha & \text { Polarizabilidad molecular/Energía libre de Helmholtz reducida } \\ \beta & \text { Factor acoplamiento cavidad } \\ \delta & \text { Efecto pelicular/Densidad reducida de mexcla } \\ \delta_{\mathrm{e}} & \text { Ángulo de pérdidas dieléctricas } \\ \varepsilon & \text { Permitividad F m-1 } \\ \varepsilon_{0} & \text { Permitividad en el vacío } 8.854 \times 10-12 \mathrm{~F} \mathrm{~m}-1 \\ \varepsilon_{\mathrm{r}} & \text { permitividad relativa } \\ \theta & \text { Coordenada cilíndrica } \\ \kappa & \text { Constante dieléctrica } \\ \lambda & \text { Longitud de onda } \\ \mu & \text { Potencial químico/Permeabilidad magnética } \mathrm{H} \text { m-1/Momento } \\ \mu_{0} & \text { dipolar permanente de una molécula } \\ \mu_{\mathrm{r}} & \text { Permeabilidad en el vacío } 4 \pi \cdot 10^{-7} \mathrm{NA}^{-2}\end{array}$




$\begin{array}{ll}\pi & \text { Número de fases de una mezcla/Número pi } 3.14159 \\ \rho & \text { Densidad molar/Densidad eléctrica } \\ \sigma & \text { Conductividad eléctrica } \\ \tau & \text { Temperatura reducida } \\ \phi_{\mathrm{i}} & \text { Coeficiente de fugacidad de la sustancia i } \\ \omega & \text { Frecuencia angular rad s-1 }\end{array}$

\section{Subíndices}

$\begin{array}{cl}\text { O } & \text { Vacío/Resonancia/Referencia } \\ \text { C } & \text { Capacidad eléctrica } \\ \text { c } & \text { Cavidad/propiedad crítica } \\ \text { d } & \text { Dieléctrico } \\ \text { g } & \text { Vapor saturado } \\ \text { I } & \text { Líquido saturado/Pérdidas eléctricas } \\ \mathrm{nmp} & \text { Condiciones de contorno } \\ \mathrm{r} & \text { Reducida } \\ \mathrm{s} & \text { Muestra } \\ \mathrm{i}, \mathrm{j} & \text { Componentes } \mathrm{i}, \mathrm{j}\end{array}$

\section{Superíndices}

"

0 ideal

$r \quad$ Comportamiento residual/reducido

$\pi \quad$ Número de fases de una mezcla

\section{Abreviaturas}

CCS Captura y almacenamiento de carbono

CEM Centro Español de Metrología

EoS Ecuación de estado

GERG Grupo Europeo de Investigación del Gas (Groupe Européen de Recherches Geziéres)

ITS-90 Escala internacional de temperaturas de 1990

ISO International Organization for Standardization 


$\begin{array}{cl}\text { pVT } & \text { Presión Volumen Temperatura } \\ \text { RLC } & \text { Circuito paralelo equivalente } \\ \text { TE } & \text { Modo transversal eléctrico } \\ \text { TERMOCAL } & \text { Termodinámica y Calibración } \\ \text { TEM } & \text { Modos transversales magnéticos y eléctricos } \\ \text { TM } & \text { Modo transversal magnético } \\ \text { VNA } & \text { Analizador vectorial de redes } \\ \text { PREoS } & \text { Peng Robinson ecuación de estado } \\ \text { PRWSEoS } & \text { Peng Robinson Wong \& Sandler ecuación de estado } \\ \text { Compuestos químicos } \\ \mathrm{CH}_{4} & \text { Metano } \\ \mathrm{C}_{2} \mathrm{H}_{6} & \text { Etano } \\ \mathrm{C}_{3} \mathrm{H}_{8} & \text { Propano } \\ \mathrm{CO}_{\mathrm{CO}} & \text { Monóxido de carbono } \\ \mathrm{CO}_{2} & \text { Dióxido de carbono } \\ \mathrm{H}_{2} & \text { Hidrógeno } \\ \mathrm{N}_{2} & \text { Nitrógeno } \\ \mathrm{O}_{2} & \text { Oxígeno } \\ \mathrm{PTFE} & \text { Politetrafluoroetileno }\end{array}$





\section{Índice de Tablas}

Tabla 5.1 Tabla de incertidumbre de medidas para la determinación de la curva de vaporización del $\mathrm{CO}_{2}$ en el intervalo de temperaturas de (273.35-302.35) K y presiones (3.502-7.077) MPa. Factor de cobertura $k=2$.

Tabla 5.2 Tabla de incertidumbre para las medidas del punto crítico en el intervalo de temperaturas de (303.36-304.04) K y presiones (7.238-7.360) MPa. Factor cobertura $\mathrm{k}=2$.

Tabla 5.3 Datos experimentales de los puntos de burbuja, rocío y frecuencia de resonancia del dióxido de carbono con una incertidumbre expandida $(k=2)$ en $U(T)=0.05 \mathrm{~K} ; \mathrm{U}(\mathrm{p})=0.006 \mathrm{MPa}$. Presiones calculadas mediante la ecuación de estado de Span \& Wagner [16].

Tabla 5.4 Datos experimentales de los puntos de burbuja, rocío y frecuencia de resonancia del dióxido de carbono en las inmediaciones del punto crítico con una incertidumbre expandida $(k=2)$ en $U(T)=0.05 \mathrm{~K} ; \mathrm{U}(\mathrm{p})=0.010 \mathrm{MPa}$. Presiones calculadas mediante la ecuación de estado de Span \& Wagner [16]. 98

Tabla 5.5 Valores calculados del punto crítico, y su correspondiente incertidumbre expandida $(k=2)$, comparada con la literatura.

100

Tabla 5.6 Coeficientes de ajuste y su desviación estándar $\sigma$ del polinomio de ajuste en la determinación de la $T_{c}$ y $P_{c}$ con respecto a la frecuencia.

100

Tabla 5.7 Valores de densidades de polarización eléctrica $\mathrm{P} / \rho$ y valores de constante dieléctrica $(\varepsilon)$ obtenidos por medio del método establecido por Harvey y Lemmon [21].

102

Tabla 6.1 Composición gravimétrica (fracción molar) e incertidumbre expandida en la composición $U(x)$ con $(k=2)$ de las mezclas investigadas. $\quad 108$

Tabla 6.2 Lista de proveedores y pureza las sustancias empleadas para la elaboración de las mezclas estudiadas en esta tesis doctoral. 
Tabla 6.3 Datos experimentales de los puntos de burbuja, rocío y frecuencia de resonancia del sistema $(0.40064) \mathrm{CO}_{2}+(0.59936) \mathrm{CH}_{4}$ con una incertidumbre expandida $(k=2)$ en $U(T)=0.05 K ; U(p)=0.010 \mathrm{MPa}$. Presiones calculadas mediante la ecuación de estado de GERG 2008 [4].

Tabla 6.4 Datos experimentales de los puntos de burbuja, rocío y frecuencia de resonancia del sistema $(0.60162) \mathrm{CO}_{2}+(0.39837) \mathrm{CH}_{4}$ con una incertidumbre expandida $(k=2)$ en $U(T)=0.05 K ; U(p)=0.010 \mathrm{MPa}$. Presiones calculadas mediante la ecuación de estado de GERG 2008 [4].

125

Tabla 6.5 Datos experimentales de los puntos de burbuja, rocío y frecuencia de resonancia del sistema (0.60053) $\mathrm{CO}_{2}+(0.39947) \mathrm{C}_{3} \mathrm{H}_{8}$ con una incertidumbre expandida $(k=2) U(T)=0.05 K ; U(p)=0.010 \mathrm{MPa}$. Presiones calculadas mediante la ecuación de estado de GERG 2008 [4].

Tabla 6.6 Datos experimentales de los puntos de burbuja, rocío y frecuencia de resonancia del sistema $(0.79777) \mathrm{CO}_{2}+(0.20223) \mathrm{C}_{3} \mathrm{H}_{8}$ con una incertidumbre expandida $(k=2)$ en $U(T)=0.05 K ; U(p)=0.010 \mathrm{MPa}$. Presiones calculadas mediante la ecuación de estado de GERG 2008 [4].

Tabla 6.7 Datos experimentales de los puntos de burbuja, rocío y frecuencia de resonancia del sistema (0.49905) $\mathrm{CO}_{2}+(0.50095) \mathrm{N}_{2}$ con una incertidumbre expandida $(k=2)$ en $U(T)=0.05 K ; U(p)=0.010 \mathrm{MPa}$. Presiones calculadas mediante la ecuación de estado de GERG 2008 [4].

Tabla 6.8 Datos experimentales de los puntos de burbuja, rocío y frecuencia de resonancia del sistema $(0.79777) \mathrm{CO}_{2}+(0.20222) \mathrm{N}_{2}$ con una incertidumbre expandida $(k=2)$ en $U(T)=0.05 K ; U(p)=0.010 \mathrm{MPa}$. Presiones calculadas mediante la ecuación de estado de GERG 2008 [4].

Tabla 6.9 Tabla resumen de las incertidumbres expandidas ( $k=2$ ) de los sistemas estudiados en esta tesis doctoral. 
Tabla 6.10 Tabla de incertidumbre para la determinación del punto de burbuja del sistema (0.40064) $\mathrm{CO}_{2}+(0.59936) \mathrm{CH}_{4}$ con una incertidumbre expandida $(k=2)$ en la isoterma $T=233.35 \mathrm{~K}$.

Tabla 6.11 Resultado de la predicción de la ecuación de estados de Peng \& Robinson [17] con reglas de mezcla de Wong \& Sandler [18]. 



\section{Índice de Figuras}

Figura 2.1 Tipos básicos de comportamiento de fase de fluido de acuerdo a la clasificación de P. H. Van Koynenburg y Scott [28].

Figura 2.2 Relación entre la corriente de carga y corriente de pérdida. Circuito equivalente (a); representación vectorial de las corrientes (b) y representación vectorial de la densidad de carga (c) [31].

Figura 2.3 Comportamiento dieléctrico de una sustancia pura polar en función de la frecuencia [34].

Figura 2.4 Símil óptico de los parámetros de dispersión.

Figura 2.5 Red de dos puertos con voltajes y corrientes definidas [31]. 28

Figura 3.1 Diagrama de clasificación de métodos experimentales [39]. 37

Figura 3.2 Capacitor coaxial cilíndrico de Straty \& Younglove [45]. 39

Figura 3.3 capacitor de platos paralelos de Straty-Younglove [46]. 40

Figura 3.4 Conjunto celda de medida y cavidad de microondas (izquierda) y celda de medida de zafiro (derecha) de Rogers et al. [10].

Figura 3.5 Circuito de microondas de Fogh y Rasmussen [11]. 42

Figura 3.6 Cavidad resonante reentrante de Goodwin et al. [14]. 43

Figura 3.7 Resonador cuasi esférico resonante de Underwood et al. [54]. 44

Figura 3.8 Cavidad cilíndrica resonadora en microondas de Ewing y Royal [55].

Figura 3.9 Cavidad resonante de Kapilevich et al.[56,57].

Figura 3.10 Cavidad cilíndrica resonante con tubo de PTFE de Kawabata et al. [61].

Figura 3.11 Cavidad cilíndrica de Kanpan et al. [62]

Figura 3.12 Geometría de una cavidad cilíndrica y distribución del campo eléctrico para modos resonantes con $l=1$ y $l=2$. 
Figura 3.13 Distribución de los campos eléctricos $(E)$ y magnéticos $(H)$ de la cavidad cilíndrica resonante.

Figura 3.14 Circuito eléctrico equivalente con cavidad acoplada. 62

Figura 3.15 Diagrama de bloques con la cavidad desacoplada. 62

Figura 3.16 Representación lorenziana del coeficiente de transmisión complejo S21 frente a la frecuencia f datos experimentales (- - ) curva ajustada $(-)$. 66

Figura 4.1 Vista de sección de la cavidad cilíndrica en microondas. $\quad 73$

Figura 4.2 Vista de sección del soporte del cable coaxial.

Figura 4.3 Vista de sección del sistema de sujeción del tubo de zafiro. 76

Figura 4.4 Diagrama esquemático del equipo experimental y equipos auxiliares empleados en esta Tesis Doctoral.

Figura 4.5 Vista frontal (izquierda) y trasera (derecha) del equipo experimental empleado en esta Tesis Doctoral. 78

Figura 4.6 Panel de control automático para en funcionamiento de las bombas ISCO.

Figura 4.7 Panel para el control automático del VNA y adquisición de datos experimentales.

Figura 4.8 Panel para el control automático del baño termostático Fluke. 83

Figura 5.1 Presión (o) y frecuencia normalizada ( $\square$ ) frente a la frecuencia de resonancia en la determinación del punto de burbuja a 298.35K.

Figura 5.2 Presión (o) y frecuencia normalizada ( $\square$ ) frente a la frecuencia de resonancia en la determinación del punto de rocío a 298.35K.

Figura 5.3 Resultados experimentales en el rango de temperaturas de $273.35 \mathrm{~K}$ a $304.04 \mathrm{~K}$ de los puntos de burbuja (o), y rocío ( $\square$ ) para el $\mathrm{CO}_{2}$, y su 
comparación con la literatura Kim et al. [72] ( $\Delta$ ); Saleh et al. [73] ( ( ); Gómez, [15] $(\square)$; datos de la referencia de Span \& Wagner (-) [16].

Figura 5.4 Desviaciones porcentuales para los datos experimentales del $\mathrm{CO}_{2}$ de los puntos de burbuja (o), y rocío () para el CO2, y su comparación con la literatura; kim et al. [72] ( $\Delta$ ); Saleh et al. [73] (৩); Gómez [15] ( $\square$ ).

Figura 5.5 Resultados experimentales en las inmediaciones del punto crítico del $\mathrm{CO}_{2}$ de los puntos de burbuja (o), y rocío () para el $\mathrm{CO}_{2}$, y su comparación con la literatura; kim et al. [72] (४); Gómez, [15] ( $\square$ ); datos desde la referencia de Span \& Wagner $(-)$ y punto crítico $(\bullet)[16]$.

Figura 5.6 Datos experimentales en las inmediaciones del punto crítico, de la temperatura de burbuja (izquierda) y rocío (derecha) (o) y a la presión de burbuja y rocío ( $\square)$ frente a la frecuencia de resonancia. Polinomio de ajuste (--). Temperatura $(\theta)$ y presión $(\square)$ crítica calculada.

Figura 5.7 Parámetro A (o) frente a la temperatura obtenido al aplicar la ecuación 3.35 mediante el método de las perturbaciones.

Figura 6.1 Frecuencia normalizada $(\square)$ y la frecuencia de resonancia $(\square)$ en función de la presión, en la determinación experimental del punto de burbuja ( $\square$ ) para el sistema (0.6) $\mathrm{CO}_{2}+(0.4) \mathrm{C}_{3} \mathrm{H}_{8}$ a $\mathrm{T}=248.15 \mathrm{~K}$.

Figura6.2 Envolvente de fases para el sistema $\left(x_{1}\right) \mathrm{CO}_{2}+\left(1-x_{1}\right) \mathrm{CH}_{4}$ ( $\left.x_{1}=0.40064\right)$ : punto de burbuja (o) rocío ( $\square$ ) esta tesis doctoral; curva calculada (), punto crítico (๑), punto cricondentherm ( $\bullet$ ), y punto cricondenbar ( ) GERG 2008 [4]; curva de ajuste de Peng-Robinson [17] \& Wong-Sandler [18] burbuja (-) y rocío (--); Donnelly et al. [80] burbuja ( () , rocío ( $\Delta$ ); Wei et al. [81] burbuja $(\diamond)$, rocío ( $\Delta$ ); Webster et al. [82] burbuja ( $\diamond)$, rocío ( $\Delta)$; Al-Sahhaf et al. [83] burbuja $(\diamond)$, rocío $(\Delta)$ y Gómez. [15] burbuja $(\diamond)$, rocío $(\Delta)$.

Figura 6.3 Desviaciones relativas respecto la ecuación GERG-2008 [4] para el sistema $\left(x_{1}\right) \mathrm{CO}_{2}+\left(1-x_{1}\right) \mathrm{CH}_{4}\left(x_{1}=0.40064\right) ;$ punto de burbuja (o) rocío (ㅁ) esta tesis 
doctoral; Donnelly et al. [80] burbuja ( () , rocío ( $\Delta)$; Wei et al. [81] burbuja ( $\diamond)$, rocío ( $\triangle$ ); Webster et al. [82] burbuja ( () , rocío ( $\Delta$ ); Al-Sahhaf et al. [83] burbuja $(\diamond)$, rocío $(\triangle)$ y Gómez. [15] burbuja $(\diamond)$, rocío $(\Delta)$.

Figura6.4 Envolvente de fases para el sistema $\left(x_{1}\right) \mathrm{CO}_{2}+\left(1-x_{1}\right) \mathrm{CH}_{4}$ ( $\left.x_{1}=0.60162\right)$; punto de burbuja (o) rocío ( $\square$ ) esta tesis doctoral; curva de ajuste (); punto crítico ( 9 ); punto cricondentherm (๑), y punto cricondenbar (๑) GERG 2008 [4]; curva de ajuste de Peng-Robinson [17] \& Wong-Sandler [18] burbuja (-) y rocío (- -); Donnelly et al. [80] rocío ( $\Delta$ ); Wei et al. [81] burbuja ( $)$ ), rocío ( $\Delta$ ); Webster et al. [82] burbuja ( () , rocío ( $\triangle$ ); Dávalos et al. [84] burbuja ( () , rocío ( $\Delta$ ); Al-Sahhaf et al. [83] burbuja ( () , rocío ( $\Delta$ ) y Gómez [15] burbuja ( () , rocío $(\Delta) .127$

Figura 6.5 Desviaciones relativas respecto la ecuación GERG-2008 [4] para el sistema $\left(x_{1}\right) \mathrm{CO}_{2}+\left(1-x_{1}\right) \mathrm{CH}_{4}\left(x_{1}=0.60162\right)$; punto de burbuja (o) rocío ( $\square$ ) esta tesis doctoral; Donnelly et al. [80] rocío ( $\Delta$ ); Wei et al. [81] burbuja ( () , rocío ( $\Delta$ ); Webster et al. [82] burbuja ( () , rocío ( $\Delta$ ); Dávalos et al. [84] burbuja ( $)$ ), rocío ( $\Delta$ ); Al-Sahhaf et al. [83] burbuja $(\diamond)$, rocío $(\Delta)$ y Gómez [15] burbuja $(\diamond)$, rocío $(\Delta)$.

128

Figura6.6 Envolvente de fases para el sistema $\left(x_{1}\right) \mathrm{CO}_{2}+\left(1-x_{1}\right) \mathrm{C}_{3} \mathrm{H}_{8}$ ( $\left.x_{1}=0.60053\right)$; punto de burbuja (o) rocío ( $\square$ ) esta tesis doctoral; curva de ajuste (); punto crítico ( ( ); punto cricondentherm ( ๑) y punto cricondenbar ( ( ) GERG 2008 [4]; curva de ajuste de Peng-Robinson [17] \& Wong-Sandler [18] burbuja (- -) y rocío (--); Niesen et al. [85] burbuja ( $\diamond)$, rocío ( $\Delta$ ); Akers et al. [86] burbuja $(\diamond)$, rocío ( $\Delta$ ); Hamam et al. [87] burbuja ( () , rocío $(\Delta)$; Kim et al. [72] burbuja $(\diamond)$, rocío $(\Delta)$

133

Figura 6.7 Desviaciones relativas respecto la ecuación GERG-2008 [4] para el sistema $\left(x_{1}\right) \mathrm{CO}_{2}+\left(1-x_{1}\right) \mathrm{C}_{3} \mathrm{H}_{8}\left(x_{1}=0.60053\right)$, punto de burbuja (o) rocío ( $\square$ ) esta tesis doctoral, Niesen et al. [85] burbuja ( () , rocío ( $\Delta$ ); Akers et al. [86] burbuja ( () , rocío $(\triangle)$; Hamam et al. [87] burbuja ( () , rocío ( $\Delta$ ); Kim et al. [72] burbuja ( () , rocío ( $\triangle)$. 
Figura 6.8 Envolvente de fases para el sistema $\left(x_{1}\right) \mathrm{CO}_{2}+\left(1-x_{1}\right) C_{3} H_{8}$ ( $\left.x_{1}=0.79777\right)$; punto de burbuja (o) rocío ( $\square$ ) esta tesis doctoral; curva de ajuste (); punto crítico (9); punto cricondentherm (9); y punto cricondenbar (9) GERG 2008 [4]; curva de ajuste de Peng-Robinson [17] \& Wong-Sandler [18] burbuja (-) y rocío (--); Akers et al. [86] burbuja ( $\diamond)$, rocío ( $\Delta$ ); Hamam et al. [87] burbuja $(\diamond)$, rocío $(\Delta)$; Kim et al. [72] burbuja $(\diamond)$, rocío $(\Delta)$.

Figura 6.9 Desviaciones relativas respecto la ecuación GERG-2008 [4] para el sistema $\left(x_{1}\right) \mathrm{CO}_{2}+\left(1-x_{1}\right) C_{3} H_{8}\left(x_{1}=0.79777\right)$; punto de burbuja (o) rocío ( $\square$ ) esta tesis doctoral; Akers et al. [86] burbuja ( $\diamond)$, rocío ( $\Delta$ ); Hamam et al. [87] burbuja ( $\diamond)$, rocío $(\Delta)$; Kim et al. [72] burbuja $(\diamond)$, rocío $(\Delta)$.

Figura6.10 Envolvente de fases para el sistema $\left(x_{1}\right) \mathrm{CO}_{2}+\left(1-\mathrm{x}_{1}\right) \mathrm{N}_{2}$ ( $\left.x_{1}=0.49905\right)$; punto de burbuja (o) rocío ( $\square$ ) esta tesis doctoral; curva de ajuste (); punto crítico (O); punto cricondentherm (9) y punto cricondenbar (O); GERG 2008 [4]; curva de ajuste de Peng-Robinson [17] \& Wong-Sandler [18] burbuja (-) y rocío (- -); Brown et al. [88] burbuja ( () , rocío ( $\Delta$ ); Fandiño et al. [89] burbuja $(\diamond)$, rocío $(\Delta)$; Al Sahhaf et al. [83] rocío $(\Delta)$.

Figura 6.11 Desviaciones relativas respecto la ecuación GERG-2008 [4] para el sistema $\left(x_{1}\right) \mathrm{CO}_{2}+\left(1-x_{1}\right) N_{2}\left(x_{1}=0.49905\right)$; punto de burbuja (o) rocío ( $\left.\left.\square\right)\right)$ esta tesis doctoral; Brown et al. [88] burbuja ( () , rocío ( $\Delta$ ); Fandiño et al. [89] burbuja $(\diamond)$, rocío $(\Delta)$; Al Sahhaf et al. [83] rocío $(\Delta)$.

Figura 6.12 Envolvente de fases para el sistema $\left(x_{1}\right) \mathrm{CO}_{2}+\left(1-x_{1}\right) \mathrm{N}_{2}$ ( $\left.x_{1}=0.79777\right)$; punto de burbuja (o) rocío ( $\square$ ) esta tesis doctoral; curva de ajuste (); punto crítico (9); punto cricondentherm (O); y punto cricondenbar (O) GERG 2008 [4]; curva de ajuste de Peng-Robinson [17] \& Wong-Sandler [18] burbuja (-) y rocío (- -); Brown et al. [88] burbuja ( () , rocío ( $\Delta$ ); Westman et al. [90] burbuja ( $\diamond)$; Fandiño et al. [89] burbuja ( $\diamond)$, rocío ( $\triangle$ ); Al Sahhaf et al. [83] burbuja $(\diamond)$, rocío $(\Delta)$. 
Figura 6.13 Desviaciones relativas respecto la ecuación GERG-2008 [4] para el sistema para el sistema $\left(x_{1}\right) \mathrm{CO}_{2}+\left(1-x_{1}\right) N_{2}\left(x_{1}=0.79777\right)$; punto de burbuja (o) rocío ( $\square$ ) esta tesis doctoral; Brown et al. [88] burbuja ( $\diamond)$, rocío ( $\Delta$ ); Westman et al. [90] burbuja $(\diamond)$; Fandiño et al. [89] burbuja ( $\diamond)$, rocío $(\Delta)$; Al Sahhaf et al. [83] burbuja $(\diamond)$, rocío $(\Delta)$.

Figura 6.14 Desviaciones relativas respecto Peng-Robinson [17] empleando las reglas de mezcla de Wong-Sandler [18] para el sistema $\left(x_{1}\right) \mathrm{CO}_{2}+\left(1-x_{1}\right) \mathrm{CH}_{4}$ $\left(x_{1}=0.40060\right)$, punto de burbuja (o) rocío ( $\square$ ) esta tesis doctoral.

Figura 6.15 Desviaciones relativas respecto Peng-Robinson [17] empleando las reglas de mezcla de Wong-Sandler [18] para el sistema $\left(x_{1}\right) \mathrm{CO}_{2}+\left(1-\mathrm{x}_{1}\right) \mathrm{CH}_{4}$ $\left(x_{1}=0.60162\right)$, punto de burbuja (o) rocío ( $\square$ ) esta tesis doctoral.

Figura 6.16 Desviaciones relativas respecto Peng-Robinson [17] empleando las reglas de mezcla de Wong-Sandler [18] para el sistema $\left(x_{1}\right) \mathrm{CO}_{2}+\left(1-x_{1}\right) C_{3} H_{8}$ $\left(x_{1}=0.60053\right)$, punto de burbuja (o) rocío ( $\square$ ) esta tesis doctoral.

Figura 6.17 Desviaciones relativas respecto Peng-Robinson [17] empleando las reglas de mezcla de Wong-Sandler [18] para el sistema $\left(x_{1}\right) C_{2}+\left(1-x_{1}\right) C_{3} H_{8}$ $\left(x_{1}=0.79777\right)$, punto de burbuja (o) rocío ( $\square$ ) esta tesis doctoral.

Figura 6.18 Desviaciones relativas respecto Peng-Robinson [17] empleando las reglas de mezcla de Wong-Sandler [18] para el sistema $\left(x_{1}\right) \mathrm{CO}_{2}+\left(1-\mathrm{x}_{1}\right) \mathrm{N}_{2}$ ( $\left.x_{1}=0.49905\right)$, punto de burbuja (o) rocío ( $\square$ ) esta tesis doctoral.

Figura 6.19 Desviaciones relativas respecto Peng-Robinson [17] empleando las reglas de mezcla de Wong-Sandler [18] para el sistema para el sistema $\left(x_{1}\right) \mathrm{CO}_{2}+\left(1-x_{1}\right) N_{2}\left(x_{1}=0.79777\right)$, punto de burbuja (o) rocío ( $\square$ ) esta tesis doctoral. 


\section{capítulo 1}

\section{Introducción}





\subsection{El problema medioambiental}

Negar que el calentamiento global está principalmente causado por las altas concentraciones de $\mathrm{CO}_{2}$ que se encuentran en la atmósfera, es querer negar una realidad que afecta a todos los seres de este planeta. Ya en Paris [1], se estableció un acuerdo avalado por 195 países por el cual se establecería un límite de calentamiento global muy por debajo de los $2^{\circ} \mathrm{C}$ sobre los niveles preindustriales. Este acuerdo permite que cada país establezca su propio plan de actuación con el fin de lograr este objetivo, en forma de contribuciones previstas determinadas a nivel nacional, para alcanzar los objetivos voluntarios de reducción de sus emisiones antropogénicas de gases de efecto invernadero.

El $78 \%$ de los gases de efecto invernadero resultan de la combustión de combustibles fósiles y de los procesos industriales [2]. El progresivo crecimiento industrial y la creciente demanda mundial de energía pueden aumentar la acumulación de emisiones de dióxido de carbono en la atmósfera. Por lo tanto, son necesarias medidas para reducir drásticamente estas emisiones. La reducción de las emisiones de dióxido de carbono mediante la captura y almacenamiento de carbono (CCS) es una tecnología que se discute a menudo [3], sin embargo, todavía existen varios desafíos técnicos para el diseño y la operación de los sistemas de $\mathrm{CCS}$. El $\mathrm{CO}_{2}$ capturado en las centrales eléctricas y en la industria es comprimido y transportado por tuberías, y luego inyectado en el sitio de almacenamiento subterráneo. Las condiciones típicas de temperatura y presión de los procesos en los que interviene el $\mathrm{CO}_{2}$ varía en función de las necesidades, pero en condiciones de CCS el rango de operación va desde los $0.05 \mathrm{MPa}$ a $20 \mathrm{MPa}$ y desde $298.15 \mathrm{~K}$ hasta $620.00 \mathrm{~K}$. Entre los lugares de almacenamiento adecuados figuran los acuíferos salinos profundos, con la mayor capacidad de almacenamiento, seguidos de los yacimientos agotados de petróleo y gas, y los mantos de carbón no explotables. 


\section{Introducción}

Para garantizar un diseño de planta eficiente y seguro, así como un funcionamiento eficaz de los procesos dentro de la cadena CCS, es de crucial importancia el conocimiento fiable de las propiedades termofísicas de las sustancias implicadas en los procesos. Como las condiciones del proceso cubren un amplio rango de presiones, temperaturas y mezclas complejas de fluidos multicomponentes, no es rentable llevar a cabo experimentos para cada combinación de parámetros. Por lo tanto, la medición de los datos termofísicos de sistemas binarios y ternarios relativamente más simples, para el rango de presión y temperatura usuales, y su uso para verificar y mejorar los modelos termodinámicos predictivos, es una forma de simplificar y abordar el problema. Con los modelos termodinámicos predictivos mejorados, se pueden utilizar datos de varios componentes para probar el modelo. Los modelos termodinámicos predictivos mejorados pueden aplicarse para mejorar el diseño del proceso, para la seguridad y la optimización a lo largo de la cadena de CCS.

Propiedades fundamentales como el equilibrio de fases de sistemas que contienen $\mathrm{CO}_{2}$ pueden ser predichas con ecuaciones multiparamétricas como por ejemplo, la ecuación de estado GERG 2008 [4], sin embargo, la precisión de estos modelos depende fuertemente de la fiabilidad de datos experimentales en la que están basados [5], debido a esto, la medida precisa de propiedades termodinámicas de sistemas en los que interviene la captura y almacenaje de $\mathrm{CO}_{2}$ es más que esencial.

Teniendo siempre en cuenta que la sostenibilidad energética y por consiguiente la medioambiental, se basa en la búsqueda de nuevas fuentes de energía que logren descender el uso abusivo de combustibles fósiles, que sean más baratas y que sus emisiones de $\mathrm{CO}_{2}$ no sean tan elevadas. Durante las últimas décadas han surgido mezclas alternativas al gas natural como es el caso del biogás [6,7]. Este gas compuesto principalmente por metano y dióxido de carbono en proporciones variables, dependiendo de la materia orgánica de la cual se ha generado, se 
obtiene a partir de fermentación anaerobia, proceso bacteriano microbiológico en ausencia de oxígeno, de la materia orgánica en digestores de biogás, con alta variabilidad en su composición.

El biogás resultante que sale de los digestores contiene además de los compuestos mayoritarios $\mathrm{CH}_{4}$ ( (50 a 60) \%) y $\mathrm{CO}_{2}$ ((40 a 50) \%), contiene otros gases tales como $\mathrm{N}_{2}, \mathrm{CO}, \mathrm{O}_{2}, \mathrm{H}_{2}, \mathrm{H}_{2} \mathrm{O} \circ \mathrm{NH}_{3}$ además de otras impurezas estas más perjudiciales tales como $\mathrm{H}_{2} \mathrm{~S}$ o siloxanos, es por ello, que antes de su integración en las redes de distribución de gas natural, el biogás bruto es necesario que se convierta en biometano mediante diferentes métodos de purificación o upgrading los cuales abarcan desde la eliminación de $\mathrm{CO}_{2}$ mediante el lavado con agua o disolventes orgánicos, carbón activado o separación por membranas. El biometano resultante tendrá una composición final de $\mathrm{CH}_{4}>95 \%, \mathrm{CO}_{2}<2 \% \mathrm{H}_{2} \mathrm{~S}<2 \mathrm{mg} \cdot \mathrm{m}^{-3} \mathrm{y}$ siloxanos inferiores a $10 \mathrm{mg} \cdot \mathrm{m}^{-3}$.[8].

En la unión europea en 2016 la generación de biogás alcanzó los 16.1 Mtoe (tonelada equivalente de petróleo), con una producción eléctrica a partir de biogás de $62.5 \mathrm{TW} \cdot \mathrm{h}$. El productor primario en biogás es hasta el momento es Alemania con una producción total de 7956.3 Mtoe, España se encuentra en la décima posición con una producción de 230.8 Mtoe [9]. Para poder contribuir a la implementación de biogás en España en las líneas de distribución, es necesario que cumplan con ciertas especificaciones en la regulación vigente, es por este motivo que el conocimiento de sus propiedades termodinámicas se hace de necesidad con el fin de proporcionar datos fiables para mejorar los modelos termodinámicos empleados para calcular y diseñar la extracción, transporte, almacenamiento, distribución y normas de calidad de las mezclas de biogás. 


\subsection{Antecedentes}

Los aparatos basados en microondas han demostrado su aplicabilidad el campo de la investigación de una amplia gama de propiedades termofísicas de sustancias puras y mezclas. Además, los resonadores en microondas han sido ampliamente caracterizados con el fin de investigar los equilibrios de fase de fluidos puros y el comportamiento $(p, T, x)$ de las mezclas de fluidos, y es este el objetivo principal de esta tesis doctoral, el de proponer un nuevo resonador en microondas con una geometría cilíndrica y un tubo en el interior de la celda, que contendrá la muestra con el fin de obtener datos experimentales de calidad y precisión.

Las técnicas en microondas fueron introducidas por primera vez por Rogers et al. [10]. Sus equipos fueron diseñados para el estudio de la transición de fase de sustancias corrosivas a temperaturas superiores a $588 \mathrm{~K}$ y presiones hasta 170 $\mathrm{MPa}$. El sistema evanescente consistía de un resonador cilíndrico operando a frecuencias de 8 a $12 \mathrm{GHz}$, el cual estaba conectado con la celda que contenía la muestra a alta presión. La cavidad y la muestra estaban separadas por una ventana de zafiro. El sistema evanescente tenía un volumen total de $40 \mathrm{~cm}^{3}$, donde el volumen de la celda que contenía la muestra ocupaba aproximadamente unos 20 $\mathrm{cm}^{3}$. La construcción permitía la penetración de la microondas a través de la ventana de zafiro hasta el fluido bajo estudio. La transición de fase fue determinada por el cambio en la frecuencia de resonancia y el factor de calidad Q. Rogers et al. [10] midieron en condiciones isotérmicas los puntos de rocío y burbuja de sustancias puras como el propano o el dióxido de carbono y de sistemas que contenían aceites con una resolución superior a $0.1 \mathrm{~K}$ y $0.07 \mathrm{MPa}$.

Fogh et al. [11] presentaron un sistema automático de volumen variable. El equipo permitía las medidas de los puntos de rocío y burbuja a temperaturas entre (253 a 473) K y a presiones entre (5 a 10) MPa. La celda que contenía la muestra poseía un volumen aproximado de $10 \mathrm{~cm}^{3}$ y se midieron las envolventes de fase de 
sistemas etano + octano. Empleando esta misma celda, Frørup et al. [12] determinaron los puntos de rocío y burbuja en los alrededores de la zona crítica de sistemas etano + heptano y etano + octano. Más tarde, Goodwin et al. [13] usaron esta mismo aparato para la determinación en condiciones isocóricas de puntos de rocío de sistemas metano + propano y, gravimétricamente, prepararon mezclas de diferentes hidrocarburos los cuales contenían metano e icosano pero de estos sistemas no hay información concernientes a las incertidumbres en las medidas de los puntos de rocío publicados. Posteriormente empleando una cavidad reentrante diseñada por Goodwin et al. [14] usada a frecuencias aproximadas a $375 \mathrm{MHz}$ determinando envolventes de fases cerca de la zona crítica en mezclas de dióxido de carbono y metano a temperaturas cercanas a $290 \mathrm{~K}$ y presiones en un rango de 5 a $7 \mathrm{MPa}$.

Como punto de partida para elaborar esta tesis doctoral se ha recurrido al trabajo de tesis doctoral de Ángel Gómez [15], donde se describe un primer prototipo de la celda de medida empleado para la determinación de envolventes de fases a alta presión. Con este primer prototipo se ha logrado determinar la envolvente de fases de sistemas metano + dióxido de carbono, y se ha determinado envolventes de fase de un biogás sintético y un biogás bruto obtenido directamente de la planta de biometanización de "La Paloma".

\subsection{Objetivos}

La investigación presentada en esta tesis doctoral, se enmarca dentro de la línea de investigación que el grupo TERMOCAL (Laboratorio de Metrología y Calibración de la Universidad de Valladolid) desarrolla en su laboratorio, dicho laboratorio se especializa en medidas de propiedades termofísicas y termodinámicas, con muy baja incertidumbre, de fluidos de interés industrial. 
Con esto los principales objetivos de esta tesis doctoral son:

- Diseñar una nueva cavidad cilíndrica basada en la propuesta por Ángel Gómez [15] con la que poder realizar medidas experimentales en el equilibrio de fases a altas presiones sin los inconvenientes que el anterior equipo poseía.

- Validar dicha técnica con una sustancia de la que se conozcan muy bien sus propiedades termodinámicas (dióxido de carbono) y comparar los resultados de desviaciones obtenidas experimentalmente con la ecuaciones de estado, Span \& Wagner [16].

- Conocimiento de la envolvente de fases de las mezclas binarias formadas por $\left(\mathrm{CO}_{2}+\mathrm{CH}_{4}\right),\left(\mathrm{CO}_{2}+\mathrm{C}_{3} \mathrm{H}_{8}\right)$ y $\left(\mathrm{CO}_{2}+\mathrm{N}_{2}\right)$ a diferentes composiciones en fracción molar de $\mathrm{CO}_{2}$ en el rango de temperatura de $229.15 \mathrm{~K}$ hasta el punto de máxima temperatura (cricondentherm).

- Elaborar el procedimiento de cálculo para el tratamiento de los datos experimentales y el análisis de las incertidumbres siguiendo el estado del arte de la bibliografía.

- Validación de las ecuaciones de estado empleadas en la industria del gas por medio de la ecuación GERG 2008 [4] y mediante unas de las ecuaciones cúbicas de estado más usadas en tratamiento termodinámico como es las de Peng-Robinson [17] empleando las reglas de mezcla de Wong-Sandler [18], mediante el estudio comparativo de las desviaciones relativas respecto a los resultados experimentales.

- Contribución en la base de datos termodinámica con nuevos datos experimentales de calidad y precisos para el mejor desarrollo de los modelos empleados. 


\subsection{Estructura de la tesis}

La estructura general de la presente tesis doctoral es el siguiente:

El capítulo 1 presenta una breve introducción de las motivaciones que dan lugar al desarrollo del trabajo, describe los objetivos que se pretenden abordar en el estudio de las mezclas y pone en contexto los antecedentes en que se basa esta técnica experimental.

El capítulo 2 describe las propiedades termodinámicas de los fluidos, se realiza un estudio de las propiedades del equilibrio, se detalla el concepto de equilibrio de fases a alta presión y se hace un breve estudio de los tipos de diagramas que los datos experimentales devuelven en función de las variables que se representan. Además de realiza un estudio las propiedades electromagnéticas de los fluidos, donde se describen los conceptos de constante dieléctrica y de red de microondas, en la que se basa la técnica experimental desarrollada.

En el capítulo 3 se detalla una revisión bibliográfica de las técnicas experimentales en las que está basado el equipo experimental presentado en esta tesis doctoral, en este capítulo, se describen el principio básico en el que se basa la celda de medida y las características peculiares que hace de este equipo una técnica novedosa.

El capítulo 4 presenta el equipo experimental donde se realiza una descripción de la técnica presentada, se detallan las partes de las que está compuesto el equipo especialmente de la celda de medida, por último, se detalla el software empleado para la adquisición de los datos experimentales.

En el capítulo 5 se presentan los resultados obtenidos al validar la técnica experimental con $\mathrm{CO}_{2}$ describiendo el procedimiento establecido para la adquisición de los datos experimentales, se detalla el procedimiento de cálculo de las incertidumbres mediante la guía para la expresión de la incertidumbre de medida (JCGM 106 2012) [19]. Por último se presentan los resultados 
experimentales obtenidos comparando estos con la ecuación de estado Span \& Wagner [16] implementada en el software NIST REFPROP [20], y con la literatura encontrada.

En el capítulo 6 se muestran los resultados obtenidos en la determinación de la envolvente de fases de seis mezclas binarias formadas por $\mathrm{CH}_{4}, \mathrm{C}_{3} \mathrm{H}_{8}$ y $\mathrm{N}_{2}$ a diferentes composiciones de $\mathrm{CO}_{2}$. Todas estas mezclas han sido estudiadas en el rango de temperatura dentro de los límites del baño termostático que establecen como límite inferior $233.15 \mathrm{~K}$ llegando hasta la zona de máxima temperatura de cada uno de los sistemas (cricondentherm), las medidas experimentales también se han realizado en las proximidades al punto crítico y en la zona de condensación retrógrada. Se describe la manera en la que las muestras han sido preparadas y el procedimiento llevado a cabo para obtener los datos de cada uno de los sistemas estudiados. Se realiza además un estudio de la incertidumbre mediante la guía para la expresión de la incertidumbre de medida" (JCGM 106 2012) [19], comparando los datos experimentales con la ecuación de estado de GERG-2008 [4] implementada en la base de datos en el NIST REFPROP [20], además los datos experimentales obtenidos se han correlacionados con las propiedades dieléctricas empleando para ello el procedimiento establecido por Harvey y Lemmon [21] y se presentan los resultados de la modelización mediante la ecuación de estado de Peng-Robinson [17] empleando las reglas de mezcla de Wong-Sandler [18].

El capítulo 7 enumera las conclusiones obtenidas y las aportaciones que ha dado lugar este trabajo.

El capítulo 8 se presenta un breve resumen en ingles con el fin de cumplir con los requisitos de un trabajo de tesis doctoral con mención internacional 
CAPítulo 2

Propiedades termodinámicas y electromagnéticas de los fluidos 



\subsection{Introducción}

El estado termodinámico de un fluido, viene determinado por su presión, volumen y temperatura. En general, estas cantidades no pueden variar todas ellas independientemente. En términos matemáticos, existe una relación entre estas cantidades, que puede representarse simbólicamente como $V=f(p, T, m)$. En problemas más generales se necesitan otras variables de estado, como pueden ser la magnetización o la polarización eléctrica, en esta tesis doctoral nos centraremos en las propiedades del equilibrio de fases y en las propiedades electromagnéticas, ya que en esta última se basa la técnica experimental desarrollada. Dentro de las propiedades electromagnéticas se prestará principal atención en las propiedades de los dieléctricos, esto es debido a que la susceptibilidad magnética de las sustancias que se estudian en esta tesis doctoral puede considerarse despreciable frente a la permitividad dieléctrica.

La relación entre las propiedades volumétricas en sus diferentes fases y las propiedades dieléctricas, van a ser estudias en esta tesis doctoral y cómo pueden ser relacionadas con la frecuencia de resonancia. En este capítulo, se presentará el marco teórico relacionado con las propiedades del equilibrio y cuáles son las ecuaciones que rigen su comportamiento, además se realizará un estudio de las propiedades de los dieléctricos y su relación con los fluidos, estudiando los fenómenos de polarización de estos. Finalmente, se realizará un estudio de la relación existente entre las propiedades volumétricas y dieléctricas y cómo estas dos se correlacionan con los cambios en la frecuencia. 


\subsection{Propiedades del equilibrio}

En termodinámica, donde la atención se enfoca siempre sobre cierta cantidad de materia, una situación de equilibrio significa que no hay cambio en las propiedades del material con el tiempo. En la realidad, un verdadero estado de equilibrio probablemente nunca se alcanza debido a las continuas variaciones en los alrededores y a inercias en los posibles cambios.

El equilibrio requiere de un balance de todos los potenciales que pueden causar un cambio; sin embargo, la rapidez del cambio y por tanto la rapidez de aproximación al equilibrio, es proporcional a la diferencia de potencial entre el estado real y el estado de equilibrio $[22,23]$.

\subsubsection{El potencial químico}

Las propiedades macroscópicas de los fluidos homogéneos que están en el equilibrio son funciones de la presión, temperatura y la composición. En un sistema multicomponente y atendiendo al número de moles de uno de los componentes, al variar una propiedad termodinámica como, por ejemplo, la energía libre de Gibbs, magnitud extensiva de gran importancia para el estudio del equilibrio termodinámico, y permaneciendo constantes la presión, la temperatura y el número de moles del resto de los componentes, surge la definición de una nueva propiedad extensiva denominada potencial químico $\left(\mu_{\mathrm{i}}\right)$ de la especie tomada como referencia.

Considérese un sistema de una sola fase, abierto, que solamente puede realizar trabajo volumétrico contra la presión externa. En este caso, una propiedad característica como la energía de Gibbs (G) será dependiente de, 


$$
G=G\left(T, p, n_{1}, n_{2}, \ldots\right)
$$

Conocida la dependencia indicada, cualquier cambio infinitesimal de $\mathrm{G}$ puede expresarse a través de los cambios infinitesimales experimentados por las variables de las que depende.

En el caso de que sean más de dos fases, las fases múltiples a igual condición de temperatura y presión se hallarán en equilibrio cuando el potencial químico de cada especie sea el mismo en todas las fases.

Una ecuación de gran importancia en la teoría de equilibrio de fases es la denominada ecuación de Gibbs-Duhem [24]. Aunque básicamente es un enunciado matemático, su aplicación para seleccionar las funciones termodinámicas tiene profundas consecuencias. Entre otras, obliga a la dependencia de la composición con las propiedades molares parciales y permite el cálculo de la composición de una fase a partir del conocimiento de la otra, por integración de la ecuación 2.1 se llega a la ecuación generalizada de Gibbs.

$$
d G=V d p-S d T+\sum_{i=1} \mu_{i} n_{i}
$$

Partiendo de la ecuación 2.2 y haciendo uso de las relaciones de Maxwell se llega la expresión final.

$$
S d T-V d p+\sum_{i=1} n_{i} d \mu_{i}=0
$$

La ecuación 2.3 denominada de Gibbs-Duhem, se trata de una ecuación fundamental en la termodinámica de soluciones. Las ecuaciones descritas hasta este punto son suficientes para sentar las bases termodinámicas en el tratamiento 
de sistemas en equilibrio, sin embargo, el potencial químico no es una propiedad práctica bajo el punto de vista matemático, además de que en los estudios de fluidos resulta muy útil comparar las propiedades de un sistema real con las de otro ideal. No obstante, existen otras magnitudes que completan o facilitan dicho tratamiento matemático, además de permitir la comparación entre las propiedades de sistemas reales e ideales, tales como la fugacidad, la actividad y sus respectivos coeficientes.

\subsubsection{La fugacidad y el coeficiente de fugacidad}

La energía de Gibbs es una propiedad termodinámica muy importante dada su fuerte relación con la temperatura y la presión a través de la siguiente ecuación,

$$
\mathrm{dG}=-\mathrm{SdT}+\mathrm{Vdp}
$$

que aplicada a un fluido puro en condiciones isotérmicas considerando este como ideal la ecuación 2.4 nos queda de la siguiente manera.

$$
\left(\mathrm{dG}_{\mathrm{i}}\right)_{\mathrm{T}}=\mathrm{RTd}\left(\operatorname{lnp}_{\mathrm{i}}\right)
$$

La simplicidad de esta ecuación requiere de la necesidad de reemplazar la presión por una nueva propiedad, que G.N. Lewis [25] definió como fugacidad (f). La razón de esta definición, se debe a que la fugacidad para un sistema no ideal debería identificarse con la presión, por lo que la ecuación 2.5 puede escribirse de la siguiente manera,

$$
\left(\mathrm{dG}_{\mathrm{i}}\right)_{\mathrm{T}}=\mathrm{RTd}\left(\operatorname{lnf}_{\mathrm{i}}\right)
$$


donde a $\mathrm{f}_{\mathrm{i}}$, es la de fugacidad del componente puro i. Cuando la ecuación 2.6 se aplica a una mezcla, como es el caso de esta tesis doctoral la ecuación anterior puede ser reescrita de la siguiente manera.

$$
\mathrm{d} \mu_{\mathrm{i}}=\operatorname{RTd}\left(\ln \hat{\mathrm{f}}_{\mathrm{i}}\right)
$$

La interpretación de la ecuación 2.7, no permite establecer una comparación entre la consideración de la idealidad y no idealidad del fluido objeto de estudio, y como el único estado de un gas real que representa un estado de gas ideal es aquel en que la presión es nula, y aplicada a una mezcla de sustancias gaseosas, para completar la definición formal de $\mathrm{f}_{\mathrm{i}}$ se requiere de la relación.

$$
\lim _{\mathrm{p} \rightarrow 0} \emptyset_{\mathrm{i}}=1
$$

El coeficiente adimensional surgido de la ecuación 2.8 recibe el nombre de coeficiente de fugacidad $\left(\emptyset_{\mathrm{i}}\right)$ para el componente puro i, que puede ser extendido a una sustancia en la mezcla.

$$
\emptyset_{i}=\frac{\hat{\mathrm{f}}_{\mathrm{i}}}{\mathrm{y}_{\mathrm{i}} \cdot \mathrm{p}}
$$

La importancia del concepto de fugacidad se refleja en que ésta puede emplearse como criterio de equilibrio de fases, de forma similar a como ocurre para los potenciales químicos, es decir, la fugacidad $\mathrm{f}_{\mathrm{i}}$, de cualquier componente en un sistema multicomponente debe ser la misma en todas las fases $(1,2, \ldots \pi)$ que estén presente, para que el sistema se encuentre en equilibrio.

$$
\mathrm{f}_{\mathrm{i}}^{1}=\mathrm{f}_{\mathrm{i}}^{2}=\cdots \mathrm{f}_{\mathrm{i}}^{\pi}
$$




\subsubsection{Regla entre fases}

Considerando un sistema cerrado de " $\mathrm{C}$ " compuestos o sustancias y " $\mathrm{F}$ " fases, y donde cada fase puede contener a las " $\mathrm{C}$ " sustancias; la composición de cada fase vendrá dada por (C-1) variables. Si, por ejemplo, las fases se designan por $\alpha, \beta, \ldots$, las fracciones molares serán $\mathrm{x}_{\mathrm{i}}^{\alpha}, \mathrm{x}_{\mathrm{i}}^{\beta}$, etc. Así pues, incluyendo la temperatura y la presión, cada fase vendrá completamente especificada por $(\mathrm{C}+1)$ variables. Si el número de fases es " $\mathrm{F}$ ", entonces, para el sistema completo tendremos $\mathrm{F} \cdot(\mathrm{C}+1)$ variables.

Cuando el sistema está en equilibrio pueden plantearse las ecuaciones 2.11, que generalizadas a un número $\mathrm{F}$ de fases,

$$
\begin{aligned}
& T_{i}^{\alpha}=T_{i}^{\beta} \quad p_{i}^{\alpha}=p_{i}^{\beta} \quad \mu_{i}^{\alpha}=\mu_{i}^{\beta} \\
& T^{\alpha}=T^{\beta}=\ldots(F-1) \text { ecuaciones de temperatura } \\
& p^{\alpha}=p^{\beta}=\ldots(F-1) \text { ecuaciones de presión } \\
& \mu_{i}^{\alpha}=\mu_{i}^{\beta}=\ldots(F-1) \text { ecuaciones de } \mu_{i} \text { por } C \text { compuetos }
\end{aligned}
$$

de esta forma, el número de ecuaciones entre variables está dado por la expresión $(\mathrm{F}-1)(\mathrm{C}+2)$.

La diferencia entre el número total de variables menos el número de ecuaciones, no dará como resultado los grados de libertad del sistema:

$$
\mathrm{L}+\mathrm{F}=\mathrm{C}+2
$$


Los grados de libertad del sistema, L, definen el número de variables que pueden ser modificadas de forma arbitraria. Por ejemplo, para un sistema de dos componentes y dos fases, resulta el número de grados de libertad es dos, es decir, se tienen dos variables sobre las que actuar. En esta tesis doctoral las dos variables son la presión y la temperatura.

\subsubsection{Equilibrio de fases a alta presión}

El concepto de alta presión es relativo, según Prausnitz [26] hace referencia a la alta presión como aquella, lo suficientemente elevada, para promover algún cambio sobre las propiedades termodinámicas de todas las fases de las sustancias. Fue Fonseca et al. [27] quien catalogó el concepto de alta presión como toda aquella que se encuentre por encima de $1 \mathrm{MPa}$.

El propósito de los cálculos de equilibrio de fases es predecir las propiedades termodinámicas de mezclas, evitando determinaciones experimentales directas, 0 para extrapolar los datos existentes a diferentes temperaturas y presiones. Los requisitos básicos para el desempeño de cualquier cálculo termodinámico son la elección del modelo termodinámico apropiado y el conocimiento de los parámetros requeridos por el modelo.

En el caso de equilibrio de fases a alta presión, el modelo termodinámico utilizado es generalmente una ecuación de estado que es capaz de describir las propiedades de ambas fases. La ecuación de partida general es la igualdad de fugacidades de cada especie en cada fase:

$$
\hat{\mathrm{f}}_{\mathrm{i}}^{\mathrm{l}}\left(\mathrm{T}, \mathrm{p}, \mathrm{x}_{\mathrm{i}}\right)=\hat{\mathrm{f}}_{\mathrm{i}}^{\mathrm{v}}\left(\mathrm{T}, \mathrm{p}, \mathrm{y}_{\mathrm{i}}\right)
$$

o, introduciendo los coeficientes de fugacidad, ecuación 2.9. 


$$
\mathrm{p} \widehat{\emptyset}_{\mathrm{i}}^{\mathrm{v}} \mathrm{y}_{\mathrm{i}}=\widehat{\emptyset}_{\mathrm{i}}^{\mathrm{l}} \mathrm{x}_{\mathrm{i}} \mathrm{p}
$$

El problema típico al resolver fases fluidas, es establecer la estabilidad del sistema o la posibilidad de que el sistema se dividirá en dos o más fases en equilibrio.

\subsubsection{Diagrama de fases a alta presión}

De acuerdo con la clasificación de Konynenburg y Scott [28] hay seis tipos básicos de comportamiento de fase del fluido. Las proyecciones $p$ vs $T$ correspondientes se muestran en la Figura 2.1. Estas proyecciones $p$ vs T pueden complicarse aún más por la aparición de homo y heteroazeótropos.
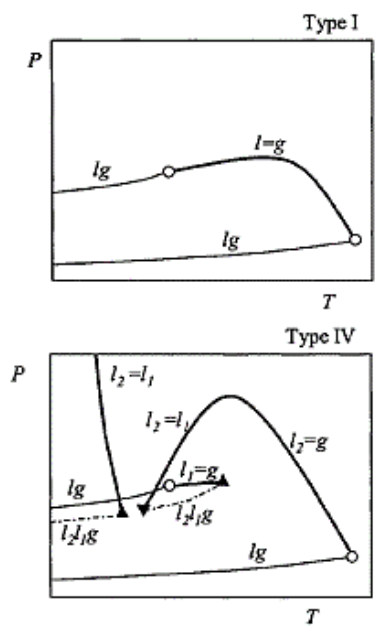
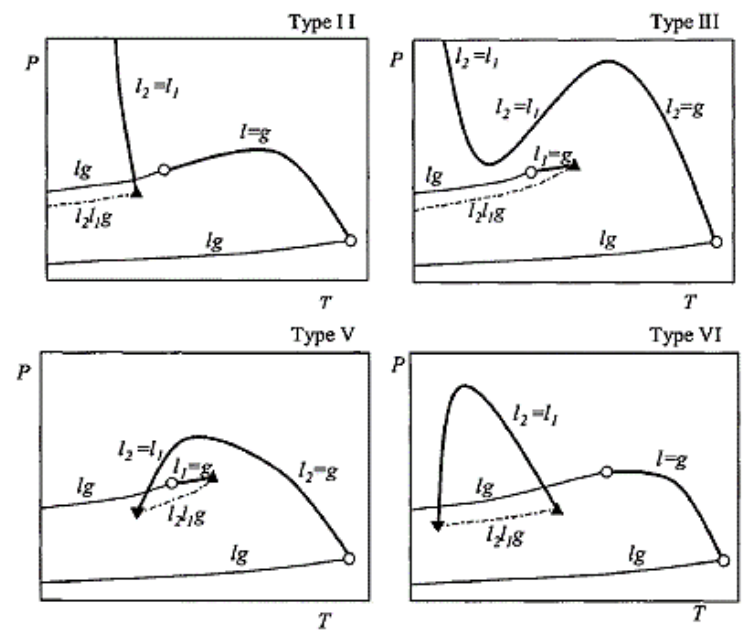

Figura 2.1Tipos básicos de comportamiento de fase de fluido de acuerdo a la clasificación de P. H. Van Koynenburg y Scott [28].

En esta tesis doctoral nos centraremos en los diagramas correspondientes a los de Tipo I también conocidos como envolventes de fase, que caracterizan el comportamiento de un fluido multicomponente a diferentes presiones $y$ 
temperaturas, este tipo de gráficas también muestra las condiciones en las cuales las fases líquidas y vapor coexisten. La envolvente de fase de cualquier sistema que se pretenda estudiar quedará completamente definida cuando se estudien una serie de puntos característicos, a saber; el punto crítico, el punto de máxima temperatura (cricondentherm) y el punto de máxima presión (cricondenbar) [29].

- Punto crítico: lugar donde las densidades de las fases líquida y vapor son iguales, además marca el límite entre las zonas de burbuja y rocío.

- "Cricondentherm": es la zona de máxima temperatura por encima de la cual no se puede formar líquido independientemente de la presión.

- "Cricondenbar": es la zona de máxima presión por la cual no se puede formar gas independientemente de la temperatura.

En 1892, J.P. Kuenen, en la universidad de Leiden, a través de su tesis doctoral en el estudio de una serie de ensayos experimentales, con el fin de comprobar la teoría de Van der Waals en mezclas binarias, observó la formación de líquido seguido de una vaporización con el consecuente aumento de la presión. A este fenómeno lo denominó punto de condensación retrógrada de primer tipo. En los yacimientos de gas condensado, cuando se reduce la presión por debajo del valor de la presión de rocío, el gas en el yacimiento empieza a transformase en líquido, formando una fase discontinua y no móvil, si la presión sigue disminuyendo el líquido que se ha formado vuelve a su estado gaseoso, este fenómeno que ocurre en fracciones de segundos es el denominado como condensación retrógrada [30]. 


\subsection{Propiedades electromagnéticas de los fluidos}

Los materiales electromagnéticos o como ocupa esta tesis doctoral, los fluidos electromagnéticos, son estudiados a escalas microscópica y macroscópicas. Nos referimos a escala microscópica, a las bandas de energía y el momento magnético de los átomos y las moléculas, mientras que, en la escala macroscópica, se estudia la respuesta general a los campos electromagnéticos [31]. Las características de los fluidos vienen determinadas por las propiedades eléctricas y en menor medida por las electromagnéticas (ferro-fluidos). Las sustancias empleadas en esta tesis doctoral no encajan en esta categoría. A escala macroscópica, las interacciones entre el fluido y los campos electromagnéticos están definidas mediante las ecuaciones de Maxwell que se muestran a continuación,

$$
\begin{gathered}
\boldsymbol{\nabla} \cdot \mathbf{D}=\rho \\
\boldsymbol{\nabla} \cdot \mathbf{B}=0 \\
\boldsymbol{\nabla} \times \mathbf{H}=\partial \mathbf{D} / \partial \mathrm{t}+\mathbf{J} \\
\boldsymbol{\nabla} \times \mathbf{E}=-\partial \mathbf{B} / \partial \mathrm{t}
\end{gathered}
$$

siendo $\mathbf{D}, \mathbf{B}, \mathbf{H}, \mathbf{J}$, y $\mathbf{E}$, los vectores de desplazamiento eléctrico, de densidad de flujo, de intensidad de campo magnético, de densidad de corriente eléctrica y de intensidad de campo eléctrico respectivamente, además $\rho$ se define como la densidad eléctrica. Con las siguientes relaciones constitutivas: 


$$
\begin{gathered}
\mathbf{D}=\varepsilon \mathbf{E}=\left(\varepsilon^{\prime}-\mathrm{j} \varepsilon^{\prime \prime}\right) \mathbf{E} \\
\mathbf{B}=\mu \mathbf{H}=\left(\mu^{\prime}-j \mu^{\prime \prime}\right) \mathbf{H} \\
\mathbf{J}=\sigma \mathbf{E}
\end{gathered}
$$

Las sustancias que se han empleado en la elaboración de esta tesis doctoral han sido consideradas como sustancias dieléctricas, es decir, que presentarán una conductividad despreciable, su permeabilidad magnética puede considerarse similar a la del vacío. La última consideración que se tiene en cuenta es que los campos electromagnéticos son relativamente débiles y que la frecuencia de microondas a la que se someten dichas sustancias no produce la ruptura del dieléctrico y la ionización de las moléculas.

Al aplicar un campo eléctrico a una sustancia pura, esta responderá de una determinada manera que puede clasificarse en dos categorías: fluidos polares y no polares [32]. Los fluidos polares son aquellos cuyas moléculas poseen un momento dipolar permanente. Estos han de ser comparados con los fluidos no polares, cuyas sus moléculas solo poseen una red de polarización eléctrica cuando un campo eléctrico externo es aplicado. Los fluidos polares poseen una elevada permitividad relativa con valores que comprenden el rango de 10-100, lo que origina dipolos permanentes en las moléculas. Los fluidos polares poseen también elevadas pérdidas dieléctricas en frecuencias de microondas, con pérdidas tangenciales dieléctricas en torno al rango de 0.1-1, derivados de la relajación de las orientaciones moleculares cuando se aplican campos eléctricos alterados.

\subsubsection{Concepto de constante dieléctrica}

La constate dieléctrica $\mathrm{k}$, o permitividad de un fluido, ecuación 2.23 , es una medida que trata la relación entre la permitividad del medio $\varepsilon$ y la del vacío $\varepsilon_{0}$ [33]. 
Esta propiedad depende del campo empleado para medirla, pues la polarización está ligada al desplazamiento de electrones e iones y estos desplazamientos siguen con mayor o menor retraso las variaciones del campo.

$$
\mathrm{k}=\frac{\varepsilon}{\varepsilon_{0}}(1+\mathrm{x})
$$

Cuando a una sustancia dieléctrica se le somete a campo electromagnético de alta frecuencia, por ejemplo microondas (como es el caso que ocupa esta tesis doctoral), las orientaciones de los dipolos no están influenciadas por el campo eléctrico, pierden la constante dieléctrica estática y provoca una disipación de energía [31].

Se puede considerar que la mayoría de los fluidos puros y sus mezclas se comportan como materiales dieléctricos en función de sus propiedades electromagnéticas. Una sustancia dieléctrica insertada en un capacitor proporciona el circuito equivalente de la figura 2.2.

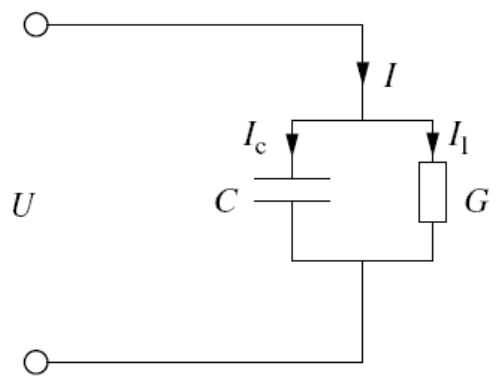

a)

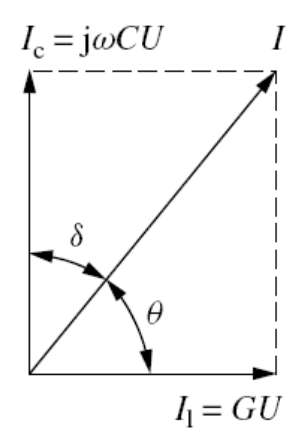

b)

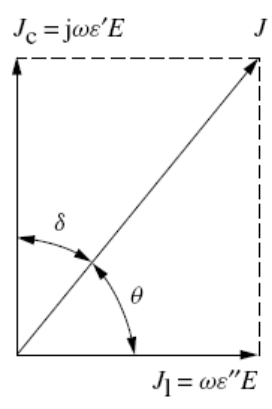

c)

Figura 2.2Relación entre la corriente de carga y corriente de pérdida. Circuito equivalente (a); representación vectorial de las corrientes (b) y representación vectorial de la densidad de carga (c) [31]. 
La corriente total está formada por dos componentes, corriente de carga $\left(I_{c}\right)$ y corriente de pérdidas $\left(l_{1}\right)$ :

$$
\mathrm{I}=\mathrm{I}_{\mathrm{c}}+\mathrm{I}_{\mathrm{l}}=\mathrm{jC} \omega \mathrm{U}+\mathrm{GU}=(\mathrm{jC} \omega+\mathrm{G}) \mathrm{U}
$$

Donde " $C$ " es la capacidad del capacitor cargado con el fluido dieléctrico, y " $G$ " es la conductancia del fluido dieléctrico. La corriente de pérdidas está en fase con el voltaje de la fuente " $U$ ". En el plano complejo mostrado en la figura 2.2 la corriente de carga está adelantada $90^{\circ} \mathrm{con}$ respecto a la corriente de pérdidas, y la corriente total está adelantada un ángulo menor a $90^{\circ}$ con respecto al voltaje de la fuente. El ángulo entre $I_{c}$ e I es denominado habitualmente ángulo de pérdida $\delta_{\mathrm{e}}$.

Con frecuencia se usa la permitividad compleja $\varepsilon^{\prime}-\mathrm{j} \varepsilon^{\prime \prime}$ para describir el efecto del fluido dieléctrico. Después de insertar un fluido dieléctrico en un capacitor, la capacitancia C viene determinada según la ecuación 2.25.

$$
\mathrm{C}=\frac{\varepsilon \mathrm{C}_{0}}{\varepsilon_{0}}=\left(\varepsilon^{\prime}-\mathrm{j} \varepsilon^{\prime \prime}\right) \frac{\mathrm{C}_{0}}{\varepsilon_{0}}
$$

Y la intensidad eléctrica viene definida por la ecuación 2.26 .

$$
I=j \omega\left(\varepsilon^{\prime}-j \varepsilon^{\prime \prime}\right) \frac{C_{0}}{\varepsilon_{0}} U=\left(j \omega \varepsilon^{\prime}+\omega \varepsilon^{\prime \prime}\right) \frac{C_{0}}{\varepsilon_{0}} U
$$

Sin embargo, tal y como se muestra en la figura 2.2 , la densidad de corriente transversal del capacitor bajo el campo eléctrico aplicado E se convierte en la ecuación 2.27 . 


$$
J=\left(j \omega \varepsilon^{\prime}+\omega \varepsilon^{\prime \prime}\right) E=\varepsilon \frac{d E}{d t}
$$

El producto de la frecuencia angular y el factor de pérdidas es equivalente a la conductividad dieléctrica: $\sigma=\omega \varepsilon^{\prime \prime}$. Esta conductividad dieléctrica agrupa todos los efectos disipativos en el fluido. Puede representar la conductividad actual causada por la migración de los portadores de carga y puede también referirse a la energía perdida asociada a la dispersión de $\varepsilon^{\prime}$, por ejemplo, la fricción que acompaña a la orientación de los dipolos.

Dos son los parámetros que describen la disipación energética en un fluido dieléctrico. La tangente de pérdidas dieléctricas viene dada por la ecuación 2.28, y el factor de potencia dieléctrico descrito por la ecuación 2.29.

$$
\begin{gathered}
\tan \delta_{\mathrm{e}}=\frac{\varepsilon^{\prime \prime}}{\varepsilon^{\prime}} \\
\cos \theta_{\mathrm{e}}=\frac{\varepsilon^{\prime \prime}}{\sqrt{\left(\varepsilon^{\prime}\right)^{2}+\left(\varepsilon^{\prime \prime}\right)^{2}}}
\end{gathered}
$$

Las ecuaciones 2.28 y 2.29 muestran que para un ángulo pequeño de pérdidas $\delta_{\mathrm{e}}, \cos \theta_{\mathrm{e}} \cong \tan \delta_{\mathrm{e}}$.

Con frecuencia se emplea la permitividad relativa o constante dieléctrica relativa, la cual es una cantidad adimensional definida por la ecuación 2.30.

$$
\varepsilon_{\mathrm{r}}=\frac{\varepsilon}{\varepsilon_{0}}=\frac{\varepsilon^{\prime}-\mathrm{j} \varepsilon^{\prime \prime}}{\varepsilon_{0}}=\varepsilon_{\mathrm{r}}^{\prime}-\mathrm{j} \varepsilon_{\mathrm{r}}^{\prime \prime}=\varepsilon_{\mathrm{r}}^{\prime}\left(1-\mathrm{j} \tan \delta_{\mathrm{e}}\right)
$$

Además de la permitividad y la permeabilidad, otro parámetro, el factor de calidad, ecuación 2.31, se emplea con frecuencia para describir un fluido electromagnético. 


$$
\mathrm{Q}=\frac{\varepsilon^{\prime \prime}}{\varepsilon^{\prime}}
$$

La figura 2.3 muestra cualitativamente el comportamiento de la permitividad relativa $\left(\varepsilon_{\mathrm{r}}^{\prime}\right.$ y $\left.\varepsilon_{\mathrm{r}}^{\prime \prime}\right)$ en función de la frecuencia [34]. Desde el punto de vista microscópico la permitividad está relacionada con varios fenómenos físicos como la conducción iónica, la relajación dipolar, la polarización atómica y la polarización electrónica. En el rango de bajas frecuencias $\varepsilon^{\prime \prime}$ está influenciada por la conductividad iónica [31]. La variación de la permitividad en el rango de microondas está causada principalmente por la relajación dipolar, y los picos de absorción en la región de infrarrojos y a frecuencias superiores, es principalmente debida a la polarización electrónica y atómica.

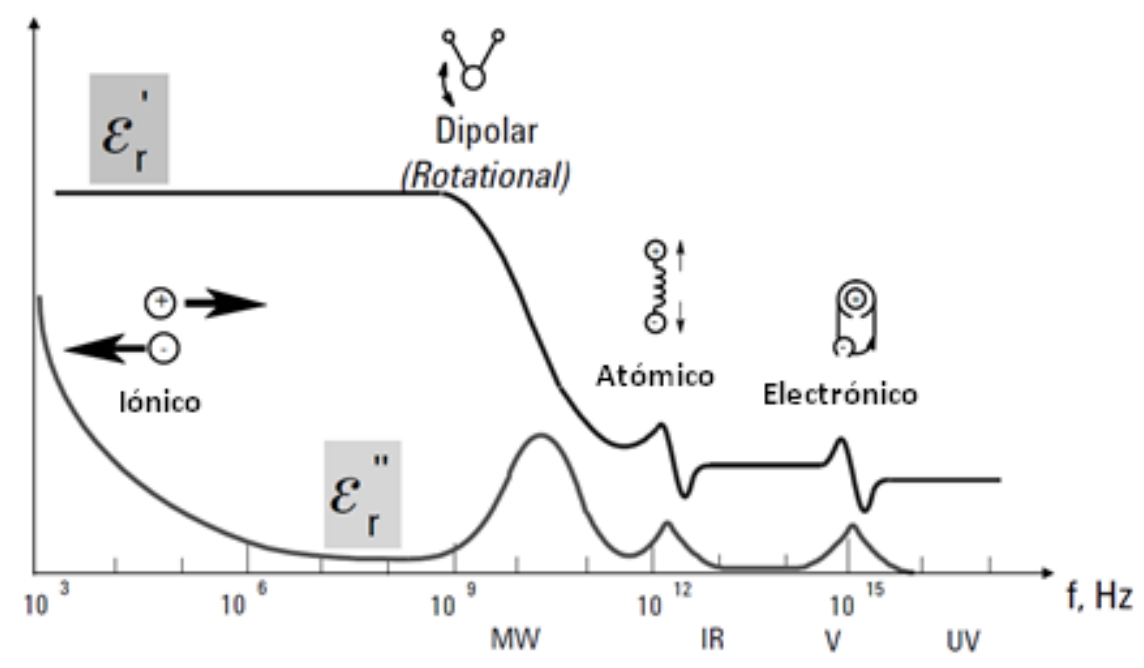

Figura 2.3Comportamiento dieléctrico de una sustancia pura polar en función de la frecuencia [34].

La polarización dipolar o por orientación surge principalmente sobre moléculas polares, que en ausencia de un campo eléctrico poseen un momento dipolar eléctrico. Cuando aplicamos un campo eléctrico, las moléculas tienden a reorientarse en la dirección de este, a altas frecuencias estas moléculas no son 
capaces de orientarse con el campo y se pierde la capacidad de polarización por orientación.

La polarización electrónica tiene lugar en átomos neutros cuando un campo eléctrico desplaza al núcleo con respecto a los electrones que le rodean. La polarización atómica sucede cuando iones positivos y negativos adyacentes se deforman bajo un campo eléctrico aplicado [31]. Realmente, la polarización electrónica y atómica son de similar naturaleza, además sus frecuencias de relajación tienen lugar en la región de infrarrojos y luz visible.

La conductividad iónica se introduce como un término añadido de pérdidas en los fluidos a bajas frecuencias y es debido, por ejemplo, por iones libres disueltos en un disolvente. En la ecuación 2.32 se observa que es inversamente proporcional a la frecuencia angular $\omega$.

$$
\varepsilon_{\mathrm{r}}^{\prime \prime}=\varepsilon_{\mathrm{rd}}^{\prime \prime}+\frac{\sigma}{\omega \varepsilon_{0}}
$$

\subsubsection{Concepto de red de microondas}

El concepto de red de microondas se desarrollada a partir de la teoría de líneas de transmisión, la cual es una herramienta muy versátil en ingeniería de microondas. El método de red de microondas estudia la respuesta de una estructura de microondas a señales externas, y es un complemento a la teoría de campos de microondas que analiza la distribución del campo en el interior de la estructura de microondas [31].

En el análisis de redes se utilizan a menudo dos conjuntos de parámetros físicos; un conjunto de parámetros son el voltaje "V" y la corriente "I". El otro conjunto de parámetros son la onda de entrada "a" y la onda de salida "b". La relación entra 
las ondas de entrada y salida son con frecuencia descritas como parámetros de dispersión "S".

Una analogía útil que permite comprender estas relaciones de potencia es mediante un símil óptico [35] tal y como puede observarse en la figura 2.4.

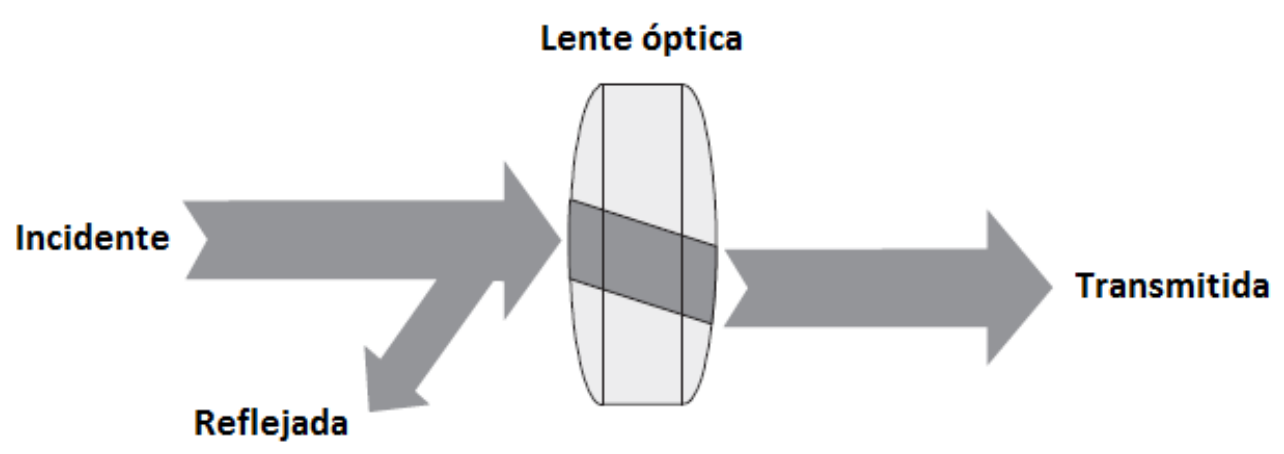

Figura 2.4Símil óptico de los parámetros de dispersión.

Parte de la luz es reflejada por la superficie de la lente, pero la mayoría de la luz atraviesa la lente. El análisis de redes en microondas consiste en medir con precisión las relaciones entre la señal incidente, la señal transmitida, y la señal reflejada.

Como se muestra en la figura 2.5, la respuesta de una red de circuitos externa puede también ser descrita mediante la entrada y salida de ondas de microondas. Las ondas de entrada al puerto 1 y 2 están denotadas como $a_{1}$ y $a_{2}$ respectivamente, y las ondas de salida desde los puertos 1 y 2 están denotadas por como $b_{1}$ y $b_{2}$. Estos parámetros $\left(a_{1}, a_{2}, b_{1}\right.$ y $\left.b_{2}\right)$ pueden ser voltaje o corriente, y en la mayoría de los casos no distinguiremos si se trata de voltaje o corriente. 


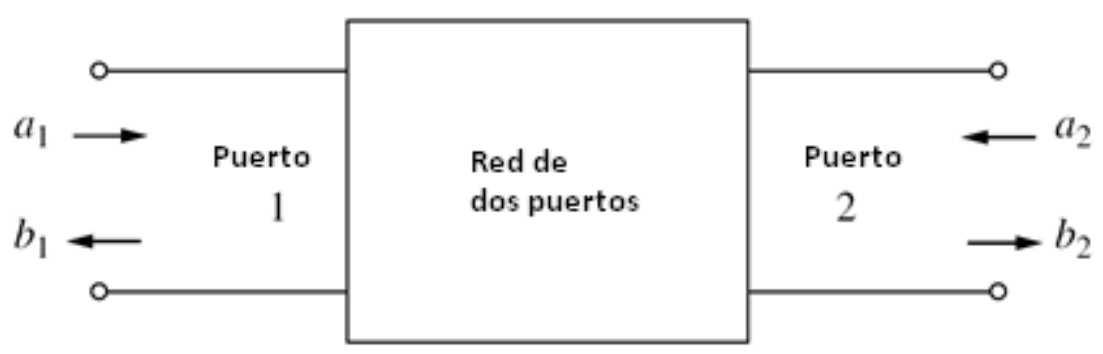

Figura 2.5Red de dos puertos con voltajes y corrientes definidas [31].

De la figura 2.5 se establece que $[a]=\left[a_{1}, a_{2}\right]^{T},[b]=\left[b_{1}, b_{2}\right]^{T}$ y la matriz de dispersión [S] se presenta en la forma de la ecuación 2.33.

$$
[S]=\left[\begin{array}{ll}
S_{11} & S_{12} \\
S_{21} & S_{22}
\end{array}\right]
$$

El parámetro de dispersión $\mathrm{S}_{\mathrm{ij}}$, cuando $\mathrm{a}_{\mathrm{i}}=0$ siendo $\mathrm{i} \neq \mathrm{j}$, teniendo en cuenta la relación $[\mathrm{b}]=[\mathrm{S}][\mathrm{a}]$, entre la onda de entrada a y la onda de salida $b$, se tiene que.

$$
\begin{gathered}
S_{j j}=\frac{b_{j}}{a_{j}} \quad(j=1,2) \\
S_{i j}=\frac{b_{i}}{a_{j}} \quad(i \neq j ; i=1,2 ; j=1,2)
\end{gathered}
$$

La ecuación 2.34 nos muestra que cuando el puerto j se conecta a la fuente y a su vez a la unidad de carga, el coeficiente de reflexión al puerto j es igual a $S_{j j}$.

La ecuación 2.35 muestra que cuando se conecta el puerto j a la fuente, y el puerto i se conecta a la unidad de carga, el coeficiente de transmisión desde el puerto j a I puerto i es igual a $\mathrm{S}_{\mathrm{ij}}$. 


\subsection{Relación entre propiedades volumétricas y dieléctricas}

Cuando se origina un cambio de fase, ya sea punto de burbuja o de rocío, este efecto trae como consecuencia una alteración en las propiedades volumétricas de la sustancia, dicha alteración está relacionada con la contante dieléctrica del material que contiene la muestra. La permitividad relativa $\varepsilon_{\mathrm{r}}$ de una sustancia es elevada si sus moléculas son polares o altamente polarizables. La relación cuantitativa entre la permitividad relativa y las propiedades eléctricas de las moléculas se obtiene considerando la polarización de un medio y se expresa mediante la ecuación de Debye.

$$
\frac{\varepsilon_{\mathrm{r}}-1}{\varepsilon_{\mathrm{r}}+2}=\frac{\rho \mathrm{P}_{\mathrm{m}}}{\mathrm{M}}
$$

Donde $\rho$ representa la densidad en masa de la muestra, $\mathrm{M}$ es la masa molar de las moléculas y $\mathrm{P}_{\mathrm{m}}$ es la polarización molar la cual se define como:

$$
\mathrm{P}_{\mathrm{m}}=\frac{\mathrm{N}_{\mathrm{A}}}{3 \varepsilon_{0}}\left(\alpha+\frac{\mu^{2}}{3 \mathrm{k}_{\mathrm{B}} \mathrm{T}}\right)
$$

donde $\alpha$ es la polarizabilidad molecular estática, y el término $\mu^{2} / 3 \mathrm{k}_{\mathrm{B}} \mathrm{T}$ proviene del momento dipolar eléctrico en presencia del campo aplicado. Cuando el momento dipolar no contribuye a la polarización [36], que se da cuando las moléculas son no polares (consideración que se tiene en cuenta en las sustancias empleadas en esta tesis doctoral) o porque la frecuencia del campo externo es tan alta que las moléculas no se pueden orientar en la dirección del campo, la constante dieléctrica puede expresarse mediante la ecuación de Clausius Mossotti para fluidos no polares [31]; la discontinuidad provocada por la variación de 
densidad en el cambio de fase, se transforma en una discontinuidad en la constante dieléctrica.

$$
\frac{\varepsilon_{\mathrm{r}}-1}{\varepsilon_{\mathrm{r}}+2}=\frac{\rho \mathrm{N}_{\mathrm{A}} \alpha}{3 \mathrm{M} \varepsilon_{0}}
$$

Estas ecuaciones en las que se relaciona la permitividad relativa $\varepsilon_{\mathrm{r}}$ de fluidos puros con las propiedades volumétricas, tienen limitaciones para pequeños valores de $\varepsilon-1$ [21]. Cuando se trabaja con fluidos no polares como son los estudiados en esta tesis doctoral, la polarización P dividida por la densidad molar $\rho$ es constante, en una primera aproximación, y es común expandirla a una serie de potencias denominada ecuación de virial dieléctrico.

$$
\mathrm{P} / \rho=\frac{\varepsilon_{\mathrm{r}}-1}{\varepsilon_{\mathrm{r}}+2}=\mathrm{A}_{\varepsilon}+\mathrm{B}_{\varepsilon} \rho+\mathrm{C}_{\varepsilon} \rho^{2}
$$

$A_{\varepsilon}$ representa el primer coeficiente del virial dieléctrico y está relacionado directamente con la polarizabilidad molecular estática $\alpha . B_{\varepsilon}$ es el segundo coeficiente del virial dieléctrico y es debido a las interacciones entre pares de moléculas. Para moléculas con un momento dipolar permanente $\mu$, se añade un término adicional contributivo a la expansión por baja densidad de la polarización molar.

$$
\mathrm{P} / \rho=\mathrm{A}_{\varepsilon}+\frac{\mathrm{N}_{\mathrm{A}} \mu^{2}}{9 \varepsilon_{0} \kappa_{\mathrm{B}} \mathrm{T}}+\mathrm{B}_{\varepsilon} \rho+\mathrm{C}_{\varepsilon} \rho^{2}
$$

Para la correlación de los datos experimentales con la expansión de la ecuación de virial dieléctrico, se modifica la expresión anterior obteniéndose la ecuación 2.41 de la siguiente manera: 


$$
\mathrm{P} / \rho=\mathrm{A}_{\varepsilon}+\mathrm{A}_{\mu} / \mathrm{T}+\mathrm{B}_{\varepsilon} \rho+\mathrm{C} \rho^{\mathrm{D}}
$$

donde $A_{\mu}=\frac{N_{A} \mu^{2}}{9 \varepsilon_{0} \kappa_{B}}$ es una forma compacta de escribir los factores en los términos dipolares, no siendo un parámetro ajustable. Los parámetros $\mathrm{A}_{\varepsilon}, \mathrm{B}_{\varepsilon} \mathrm{yC}$ son dependientes de la temperatura de la siguiente manera.

$$
\begin{aligned}
& \mathrm{A}_{\varepsilon}=\mathrm{a}_{0}+\mathrm{a}_{1}\left(\frac{\mathrm{T}}{\mathrm{T}_{0}}-1\right) \\
& \mathrm{B}_{\varepsilon}=\mathrm{b}_{0}+\mathrm{b}_{1}\left(\frac{\mathrm{T}_{0}}{\mathrm{~T}}-1\right) \\
& \mathrm{C}=\mathrm{c}_{0}+\mathrm{c}_{1}\left(\frac{\mathrm{T}_{0}}{\mathrm{~T}}-1\right)
\end{aligned}
$$

El parámetro $C$ es un parámetro empírico, y el exponente $D$ independiente de la temperatura. $T_{0}$ es una temperatura de referencia arbitraria, Harvey y Lemmon [21], proponen como valor de referencia el punto triple del agua $\mathrm{T}_{0}=273.16 \mathrm{~K}$. 

CAPÍTULO 3

\section{Técnicas experimentales}

para la determinación del equilibrio a alta presión 



\subsection{Introducción}

Cuando se desarrolla una nueva técnica o se realiza una mejora en una ya establecida, como es el caso en esta tesis doctoral, es necesario realizar una revisión bibliográfica de las técnicas experimentales que existen a fin de poder contrastar cuan necesaria es y el valor que va a tener en los datos experimentales que ésta pueda devolver, además es necesario conocer el principio de funcionamiento en el cual está basada, y su fundamento físico y matemático. La primera parte estará enfocada en una revisión general de las técnicas experimentales a alta presión que están siendo empleadas actualmente para la medida del equilibrio de fases. La segunda parte de este capítulo estará dedicada a una revisión más centrada en las técnicas en las que está basada esta tesis doctoral, así como su principio de funcionamiento y las ecuaciones que resuelven y dan sentido a los datos experimentales obtenidos.

\subsection{Técnicas experimentales para la medida del equilibrio a alta presión}

El conocimiento del equilibrio de fases a alta presión, es importante con el fin de entender los procesos que ocurren a alta presión. Esto es de vital importancia si lo que se desea es diseñar y optimizar procesos químicos y procesos de separación, otro ejemplo de la importancia de los datos del equilibrio de fases es la captura y almacenamiento de $\mathrm{CO}_{2}$, transporte y almacenamiento de gas natural, ciclos de refrigeración y bombas de calor, y en el estudio de procesos geológicos, o en la nueva química verde que emplea $\mathrm{CO}_{2}$ supercrítico y que tiene muchas aplicaciones industriales [27]. De igual manera estos datos experimentales son necesarios para el objetivo de esta tesis doctoral que es el procesamiento, enriquecimiento y almacenamiento de biogás. 
Para llevar a cabo la determinación de datos del equilibrio, se requiere, como elemento indispensable, disponer de un recinto de equilibrio, cuya configuración y principio de funcionamiento deberá estar ligado a un método determinado. La importancia del estudio de los estados del equilibrio ha incentivado el desarrollo de diferentes técnicas experimentales, así como una amplia variedad de equipos, debido a las continuas modificaciones de los ya existentes, con la finalidad de lograr un procedimiento que minimice los errores implícitos en la experimentación y las dificultades específicas propias de la determinación del equilibrio.

Debido a ello, el número de recintos de equilibrio presentes en la literatura ha aumentado considerablemente. Es por ello, que el presente apartado se dedica de forma íntegra a citar y describir el funcionamiento de los principales equipos empleados por los investigadores a lo largo de los años. Varios autores desde 1968 han realizado revisiones y estudios de los métodos experimentales publicados $[37,38,39,40,41]$. Se han empleado diferentes métodos para la obtención de datos experimentales de equilibrio de fases a alta presión, cada método tiene aplicación en diferentes condiciones de experimentación.

En el periodo de 2005-2008 Fonseca et al. [27] clasifican los métodos experimentales en dos grandes grupos dependiendo de qué forma se determinan las composiciones de las fases: métodos analíticos y métodos sintéticos, estudiando un total de 791 artículos científicos de datos experimentales de equilibrio de fases a alta presión. Fonseca et al. [39] elaboran un diagrama de clasificación de los métodos experimentales que se muestra en la figura 3.1. 

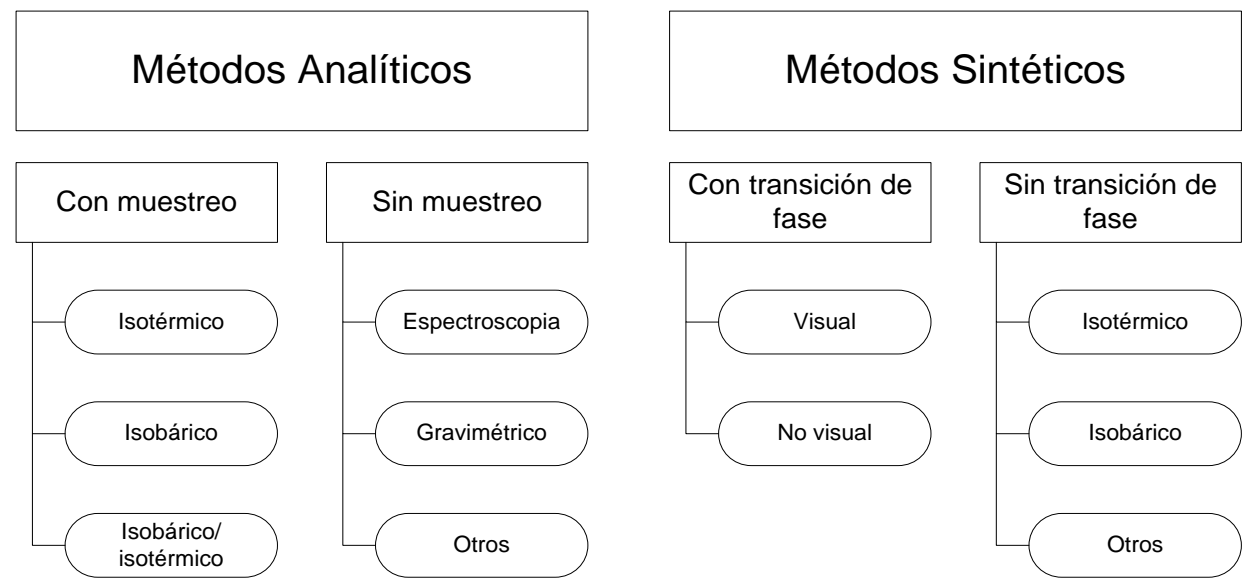

Figura 3.1Diagrama de clasificación de métodos experimentales [39].

Es prácticamente imposible obtener datos experimentales a alta presión de toda clase de sistemas utilizando una única técnica. La razón de esto es que los experimentos tienen que ser realizados en un amplio rango de presiones $y$ temperaturas, y para mezclas con componentes con diferentes propiedades físicas.

El método que se ha desarrollado en la presente tesis doctoral está clasificado dentro de los métodos experimentales como sintético con detección de cambio de fase y no visual. Otra característica es su funcionamiento en modo isotérmico. Según Fonseca et al. [27] el $62.4 \%$ de todos los artículos publicados aplicaron métodos sintéticos y el $13.4 \%$ del total eran no visuales con transición de fase. En el estudio anterior (2000-2004) de Dohrn et al. [39] era del $8.4 \%$, existiendo una tendencia creciente.

Richon y De Loos [42] han establecido una clasificación diferente en los métodos de medida en función de cómo se ha obtenido el equilibrio de fases, denominándolos métodos de circuito cerrado y de circuito abierto. Estos a su vez se han clasificado en dos subgrupos, los métodos sintéticos y analíticos. Dentro de los métodos de circuito cerrado están los estáticos y cuasi-estáticos, la mayor ventaja de estas técnicas es su simplicidad. El sistema estudiado se encierra en una 
celda de medida y se espera hasta alcanzar el equilibrio normalmente mediante un sistema de agitación. El equilibrio se comprueba mediante la estabilidad de la presión o bien mediante la constancia de la composición de las fases en función del tiempo. Las medidas se realizan generalmente a través de isotermas, ya que es más fácil reestablecer el equilibrio cambiando la presión que cambiando la temperatura, debido al espesor de las celdas de equilibrio a alta presión.

\subsection{Técnicas experimentales para la medida de propiedades dieléctricas}

Las medidas de la permitividad eléctrica relativa, de la constante dieléctrica, en función de la presión o de la temperatura $\varepsilon(\mathrm{T}, \mathrm{P})$, pueden usarse para determinar una amplio rango de propiedades termodinámicas [40]. Afsar et al. [43] en su artículo cubre la mayoría de las técnicas empleadas para la medida de la propiedades de los materiales en la frecuencia de $1 \mathrm{MHz}$ a $1500 \mathrm{MHz}$. Para el caso que nos ocupa, el uso de un tubo de muestra (cuarzo) en una cavidad resonante fue empleado por Asmussen et al. [44] para la inducción de plasma. Por otro lado, Gregory y Clark [32] han realizado una extensa revisión de los diferentes métodos para la medida de la constante dieléctrica de líquidos polares. A continuación se comentan algunas de las técnicas en las que está basado el equipo experimental presentado en esta tesis doctoral.

\subsubsection{Diseño de capacitores}

Considerando algunos ejemplos de capacitores que han sido empleados para la medida de propiedades termofísicas, nos encontramos con el trabajo desarrollado por Straty y Younglove [45] en 1970, un capacitor coaxial cilíndrico fue empleado para la medida de la constante dieléctrica del oxígeno y metano en un amplio rango de presiones y temperaturas. Los electrodos eran dos cilindros coaxiales de 
cobre, cada uno de ellos estaba soportado en cada extremo por superficies cónicas que se mantenían rígidamente, la una con respecto, a la otra mediante una barra central, todos estos elementos estaban conectados al puente.

Estos autores mostraron que la expansión térmica de su condensador cilíndrico coaxial estaba dentro del $10 \%$ de lo esperado para el cobre puro. Por lo tanto, esperaban que la contracción del capacitor bajo presión hidrostática pudiera calcularse a partir de la compresibilidad isotérmica del cobre quedando sorprendidos al encontrar evidencia de que la dependencia de la presión medida de la capacitancia era menor que la calculada.
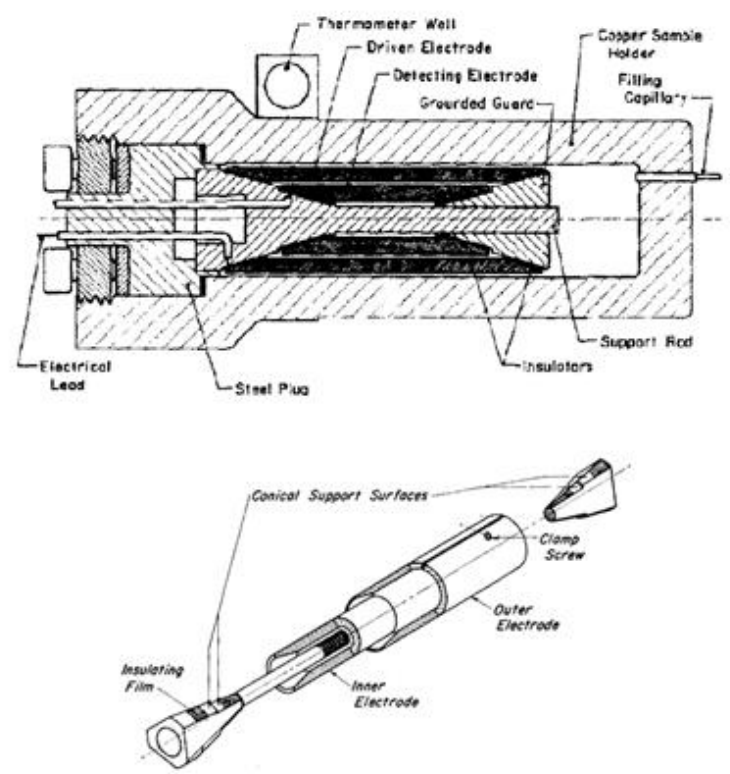

Figura 3.2 Capacitor coaxial cilíndrico de Straty \& Younglove [45].

Straty y Younglove [46] en el año 1979 presentan un capacitor de platos paralelos capaz de trabajas en el rango de frecuencias de 1-10 kHz, para el estudio de fluidos. En el diseño de estos autores, las placas del condensador, los anillos de soporte, la base y las arandelas están fabricadas en cobre. El uso de un único 
material para estos componentes provoca una disminución de la distorsión originada por la expansión térmica diferencial o de la contracción diferencial debida a la presión hidrostática. Las placas de soporte, las arandelas de ajuste y la base fueron sujetadas entre sí por tres tornillos, los cuales contribuían a la estabilidad de la estructura.
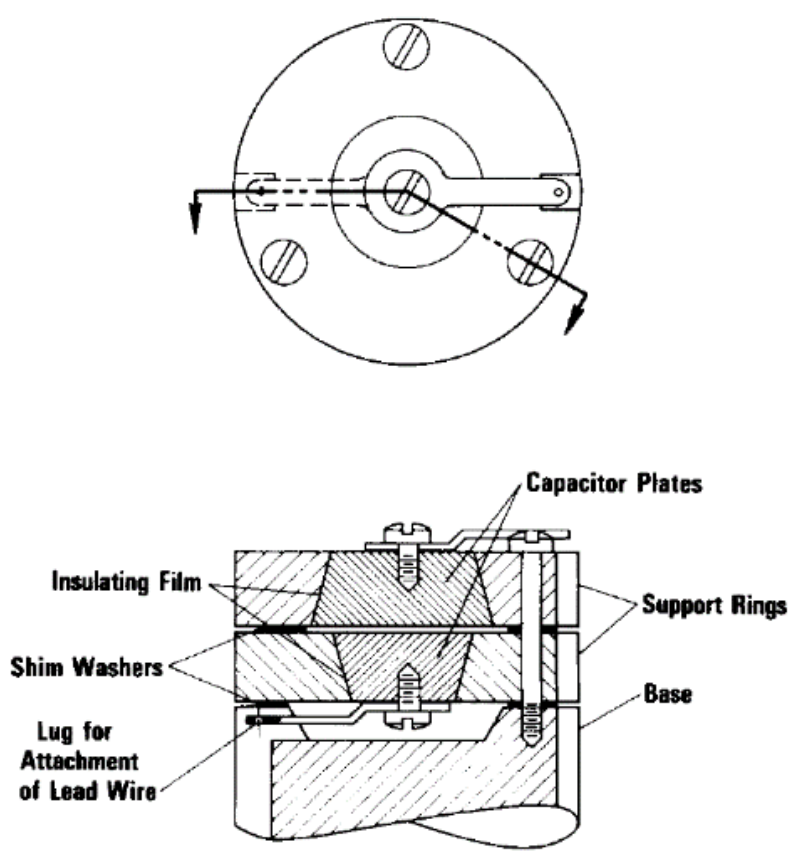

Figura 3.3 capacitor de platos paralelos de Straty-Younglove [46].

Por otro lado Goodwin y Moldover [47] empleando una cavidad como la descrita por Straty y Younglove [46], determinaron los puntos de burbuja y rocío en los alrededores de la zona crítica de mezclas binarias $\mathrm{CO}_{2}+\mathrm{C}_{2} \mathrm{H}_{6}$ a diferentes composiciones de $\mathrm{CO}_{2}$. 


\subsubsection{Cavidad cilíndrica resonante con guía evanescente}

El empleo de microondas para el estudio de sustancias tiene sus orígenes en los trabajos realizados por Rogers et al. [10] donde utilizando de una celda de medida, construida en zafiro y acoplada a la cavidad mediante una guía sonda evanescente (la cual contenía la muestra), podía determinar la transición de la fase mediante isocoras (método sintético). Mediante esta técnica se pudo determinar la transición de fases de sustancias corrosivas y el equipo se validó con la determinación del equilibrio de fases del $\mathrm{CO}_{2}, \mathrm{C}_{3} \mathrm{H}_{8}$ y una mezcla binaria de $\mathrm{CH}_{4}+\mathrm{CO}_{2}$.
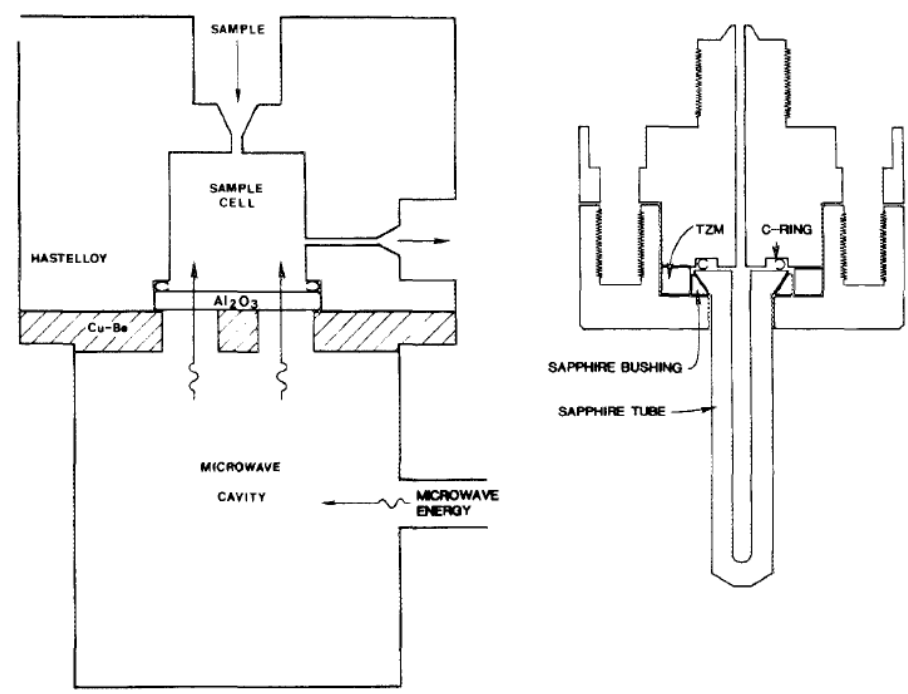

Figura 3.4 Conjunto celda de medida y cavidad de microondas (izquierda) y celda de medida de zafiro (derecha) de Rogers et al. [10].

Posteriormente Fogh y Rasmussen [11] a partir de los trabajos de Rogers et al. [10], desarrollan una nueva cavidad controlada de manera automática capaz de determinar puntos de burbuja y de rocío entre los rangos de $50-200{ }^{\circ} \mathrm{C}$ de temperatura y 50-500 bar de presión de aceites y gases. Las comunicaciones con 
el hardware de recuperación de datos se realizaron a través del bus de instrumentos HP-IB (IEEE 488), los cuales se dividieron en tres grupos. El primero de ellos consistió en un oscilador y el sintetizador que formaban parte de analizador de microondas, el segundo grupo consistió en una caja de relés que conmutaba las señales con un voltímetro digital que media las señales y las transmitía al ordenador de control. El tercer y último grupo consistía en las unidades de presentación de los datos experimentales.

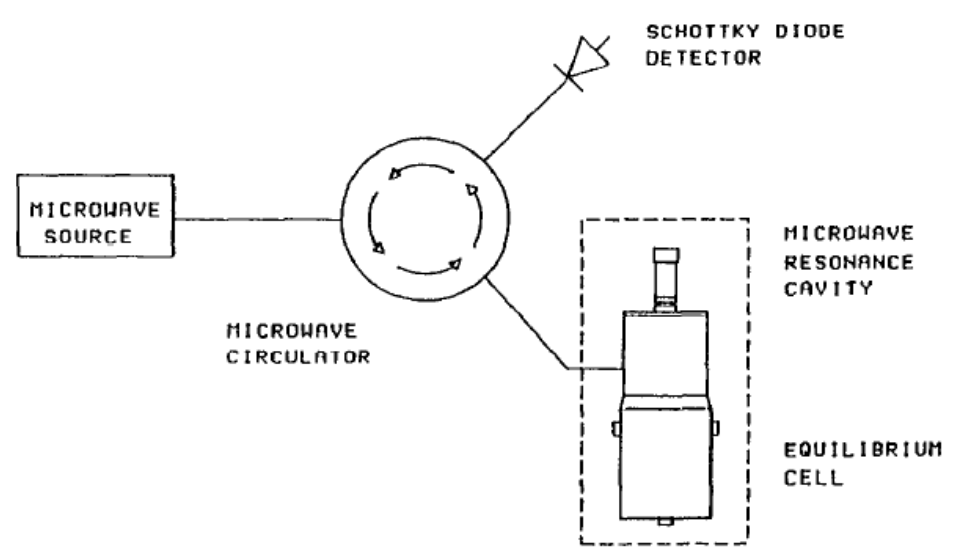

Figura 3.5Circuito de microondas de Fogh y Rasmussen [11].

Frørup et al. [12] a partir del equipo desarrollado por Rogers et al. [10] y las mejoras en cuanto a control proporcionadas por Fogh y Rasmussen [11], emplean el factor de calidad como medida para la observación en los cambios de producidos por la transición de fases cercanas al punto crítico de muestras $\mathrm{C}_{2} \mathrm{H}_{6}+\mathrm{C}_{7} \mathrm{H}_{16}$ y $\mathrm{C}_{7} \mathrm{H}_{16}+\mathrm{C}_{8} \mathrm{H}_{18}$.

\subsubsection{Cavidad reentrante resonante}

La figura 3.6 muestra una sección transversal de la cavidad reentrante resonante diseñada por Goodwin et al. [14], y usada a frecuencias aproximadas a 
$375 \mathrm{MHz}$ con el fin de detectar envolventes de fases cerca de la zona crítica en mezclas de dióxido de carbono y metano a temperaturas cercanas a $290 \mathrm{~K}$ y presiones en un rango de 5 a $7 \mathrm{MPa}$. El cuerpo del resonador está compuesto de dos partes metálicas mecanizadas. La parte inferior dispone de un cilindro hueco con una única abertura para la salida de la muestra con el fin de facilitar el estudio del equilibrio de fases en mezclas a altas presiones, y la parte superior que sirve como tapa del cilindro y dispone de un cuello que se extiende hasta la parte baja de la cavidad, que adquiere la forma de bulbo coaxial que ocupa aproximadamente $1 / 3$ de la parte inferior, estas dos partes de metal se sellaron con una junta tórica de oro. Estas dos partes de del resonador se sellan por medio de una junta tórica de oro.

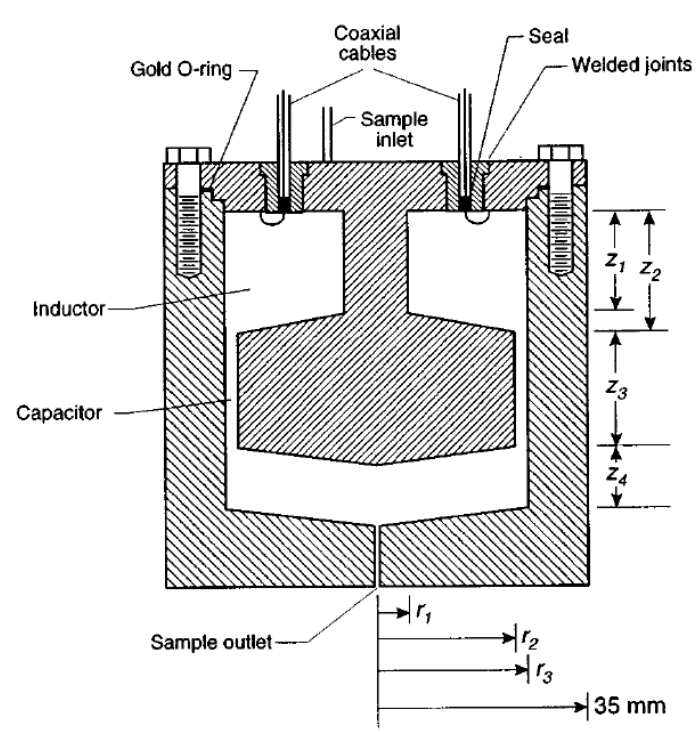

Figura 3.6Cavidad resonante reentrante de Goodwin et al. [14].

Inspirados por el trabajo de Goodwin et al. [14], May et al. $[48,49,50]$ desarrollan un nuevo aparato para la medida de comportamiento de fases donde la modificación más importante fue la incorporación de una capacidad de volumen variable en el interior del resonador. Este equipo especialmente adaptado para 
mediciones al límite de fases en gas natural, fue empleado para el estudio de la transición de fases del $\mathrm{CO}_{2}$ y de mezclas binarias de propano y $\mathrm{CO}_{2}$.

Posteriormente Huang et al. [51] emplean el diseño de Goodwin et al. [14] para el desarrollo de un higrómetro de referencia adecuado para trabajar a temperaturas superiores a las ambientales. Este resonador opera cerca de los 370 $\mathrm{MHz}$ de frecuencia, para transformar la dependencia de humedad a través de una mezcla constante de aire agua en unos cambios de frecuencia fácilmente medibles. También Kandil et al. [52,53] emplean un equipo similar para determinar la envolvente de fases de mezclas binarias que contienen metano y propano.

\subsubsection{Cavidad cuasi esférica resonante}

Underwood et al. [54] emplean un resonador cuasi esférico resonante para la determinación hidrométrica del punto de rocío. Emplean para ello los dos modos de resonancia TE y TM adaptados para una geometría esférica. El resonador es un elipsoide triaxial (figura 3.7) de aproximadamente $69 \mathrm{~cm}^{3}$ de volumen y construido en acero martensítico con un acabado superficial en oro con el fin de incrementar la conductividad eléctrica y hacerlo más resistente a la corrosión.

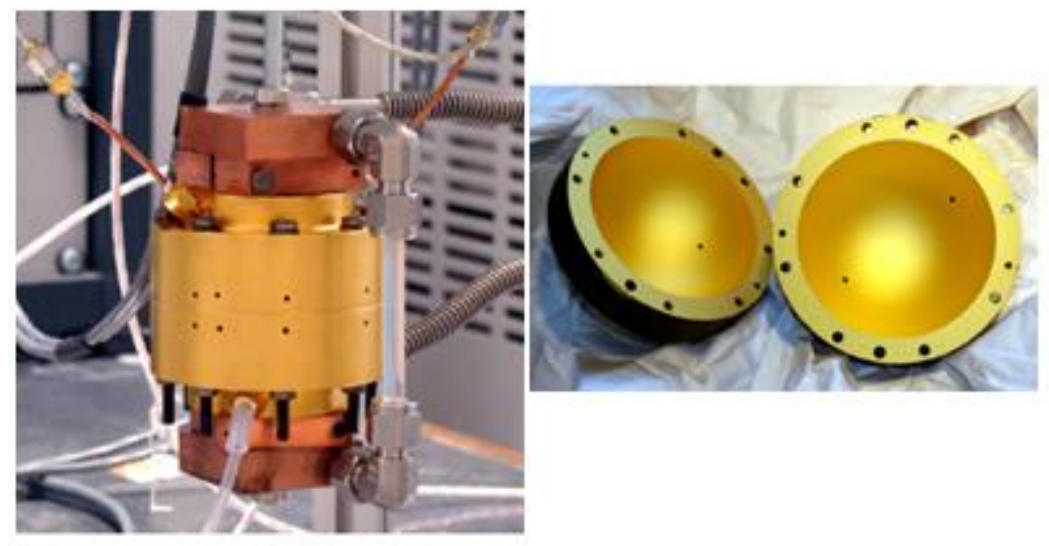

Figura 3.7Resonador cuasi esférico resonante de Underwood et al. [54]. 
En comparación con los higrómetros ópticos de punto de rocío, este dispositivo en microondas presenta significativas mejoras dado que la misma instrumentación puede ser usada de dos maneras; ya sea para medir la fracción de agua en una mezcla gaseosa lejos de las condiciones de saturación, o alternativamente, para la medición directa del punto de rocío. Esta técnica además permite determinar de manera cuantitativa el volumen y el grosor de la capa condensada.

\subsubsection{Cavidad cilíndrica resonante}

El método de perturbación en el uso de cavidades cilíndricas es una de las técnicas más empleadas. Haciendo una amplia revisión bibliográfica podemos encontrar un régimen temporal en la evolución de esta técnica experimental y como distintos autores han modificado de diversas maneras una técnica que aún se encuentra en sus inicios.

Comenzando por Ewing y Royal [55], construyen un resonador (empleando latón como material), consistente en una sección cilíndrica de extremo abierto cuyo volumen es de $5.7 \mathrm{~cm}^{3}$ y platos superior e inferior que cierran la cavidad (figura 3.8).

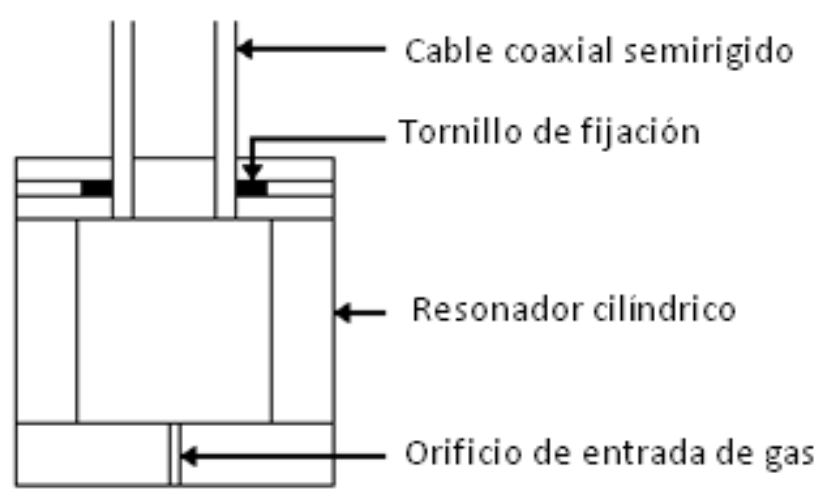

Figura 3.8Cavidad cilíndrica resonadora en microondas de Ewing y Royal [55]. 
Empleando los modos no degenerados TM010, TM011 y el modo doble degenerado TM110, desarrollan un modelo teórico con el que determinan la permitividad relativa del nitrógeno a $300 \mathrm{~K}$.

Kapilevich et al. [56,57] emplean una cavidad cilíndrica plateada, figura 3.9, con un tubo de muestra en su eje central de diferentes diámetros que varían de entre 0.25 a $0.3 \mathrm{~mm}$ para el diámetro exterior y 0.8 a $0.9 \mathrm{~mm}$ para el diámetro interior, y operando con un modo de resonancia TM101 a $3 \mathrm{GHz}$ determinan el valor de la constate dieléctrica de diversos fluidos biológicos.

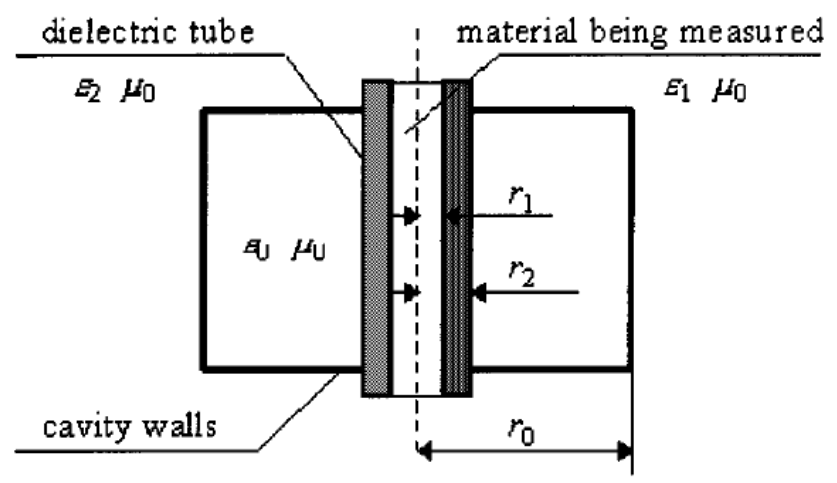

Figura 3.9Cavidad resonante de Kapilevich et al.[56,57].

Kawabata et al. [58] realizan un estudio donde analizan los errores en las medidas originados por la inserción de orificios en las cavidades resonadoras empleando un modo de resonancia TM010, estudio que ya había llevado a cabo Estin y Bussey [59] anteriormente, para ello emplean una cavidad cilíndrica construida en cobre, figura 3.10, y mediante un riguroso análisis empleando para ello el método Ritz-Galerkin de aproximación numérica, deducen que los errores relativos a la inserción de orificios por el método de perturbaciones son inferiores al 1\%. Más adelante empleando esta misma cavidad con un tubo de muestra de PTFE $[60,61]$, estudian de manera muy precisa la permitividad de fluidos tales como el agua, etanol, sake o leche. 


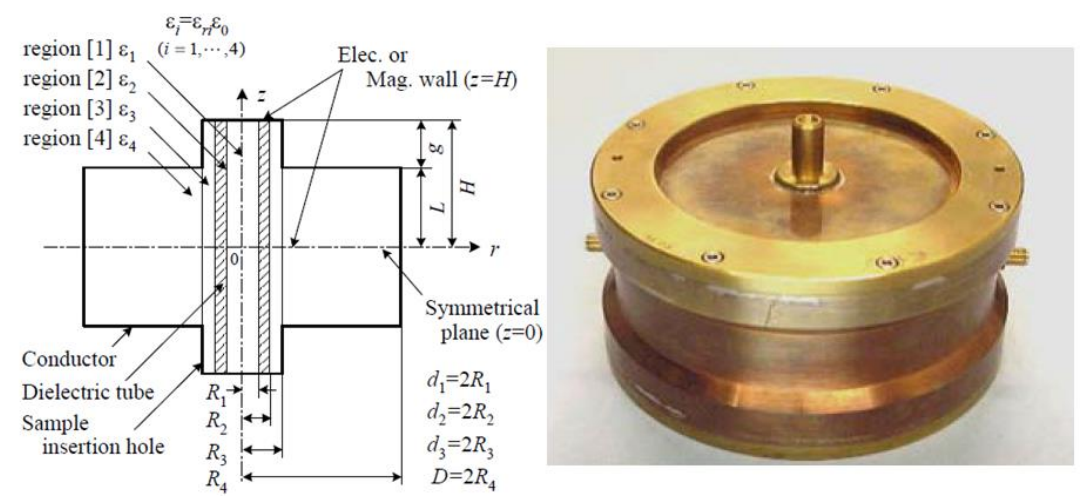

Figura 3.10 Cavidad cilíndrica resonante con tubo de PTFE de Kawabata et al. [61].

Más recientemente Kanpan et al. [62] desarrollan una cavidad cilíndrica, figura 3.11 , construida en aluminio y orificio en su eje central donde se encuentra alojado un tubo de PTFE de $0.12 \mathrm{~cm}^{3}$ de volumen para contener la muestra, emplean para la medida el modo de resonancia TM010 a $3 \mathrm{GHz}$.

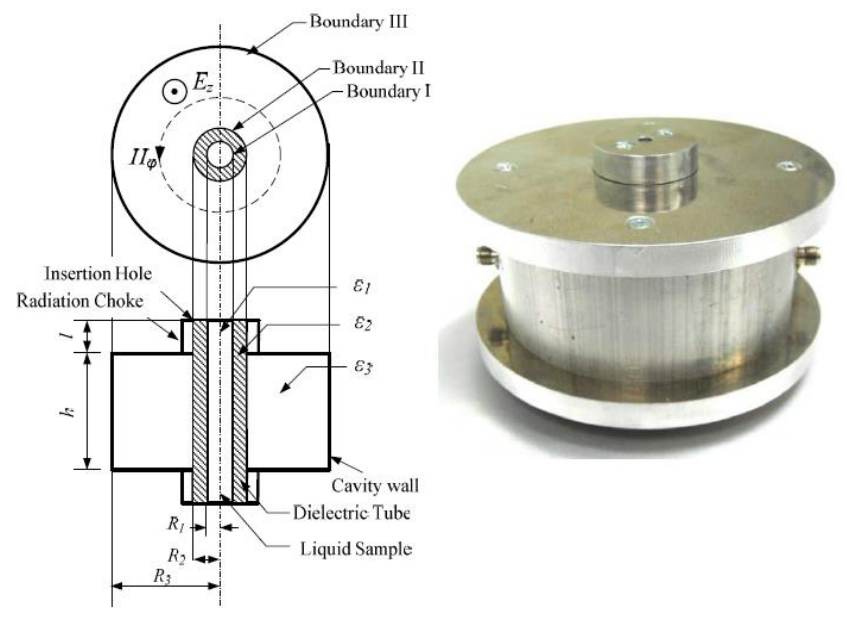

Figura 3.11 Cavidad cilíndrica de Kanpan et al. [62].

Emplean el método de perturbaciones y un análisis basado en método de RitzGalerkin, para obtener la permitividad de fluidos tales como etanol, metanol y 
agua destilada, observando que con pequeñas cantidades de muestras se puede determinar de manera precisa la permitividad dieléctrica de estos.

\subsection{Cavidad cilíndrica resonante en microondas empleada en esta tesis doctoral}

Consideremos una cavidad cilíndrica resonante como un volumen cerrado mediante una superficie conductora y cuyo interior se excita mediante un campo electromagnético, mediante diferentes modos de resonancia [63]. En la cavidad cilíndrica tienen lugar dos tipos de modos resonante: los modos transversales magnéticos ${ }^{\mathrm{TM}}$ con componente nula del campo magnético en el eje $\mathrm{z}$ del cilindro y los modos transversales eléctricos (TE) con componente nula del campo eléctrico en el eje $z$ de la cavidad cilíndrica.

Para realizar el análisis de los modos resonantes de una cavidad se considera la geometría homóloga de una sección de guía onda de transmisión cortocircuitada.
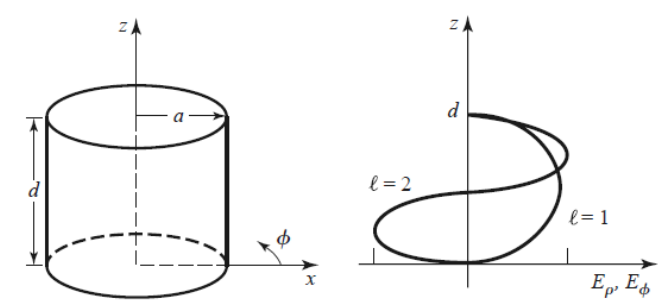

Figura 3.12 Geometría de una cavidad cilíndrica y distribución del campo eléctrico para modos resonantes con $\mathrm{l}=1$ y $\mathrm{l}=2$.

La frecuencia de resonancia de los posibles modos $\mathrm{TE}_{\mathrm{nmp}}$ para una cavidad cilíndrica como la representada se resuelven de la siguiente manera, 


$$
\begin{gathered}
\mathrm{f}_{\mathrm{nmp}}=\frac{\mathrm{c}_{0}}{2 \pi \sqrt{\varepsilon_{\mathrm{r}} \mu_{\mathrm{r}}}} \sqrt{\left(\frac{\mathrm{p}_{\mathrm{nm}}^{\prime}}{\mathrm{a}}\right)^{2}+\left(\frac{\mathrm{p} \pi}{\mathrm{l}}\right)^{2}} \\
\mathrm{n}, \mathrm{m}=1,2,3 \ldots \quad \mathrm{p}=0,1,2 \ldots
\end{gathered}
$$

y para los modos $\mathrm{TM}_{\mathrm{nmp}}$

$$
\begin{gathered}
\mathrm{f}_{\mathrm{nmp}}=\frac{\mathrm{c}_{0}}{2 \pi \sqrt{\varepsilon_{\mathrm{r}} \mu_{\mathrm{r}}}} \sqrt{\left(\frac{\mathrm{p}_{\mathrm{nm}}}{\mathrm{a}}\right)^{2}+\left(\frac{\mathrm{p} \pi}{\mathrm{l}}\right)^{2}} \\
\mathrm{n}, \mathrm{m}=1,2,3 \ldots \quad \mathrm{p}=0,1,2 \ldots
\end{gathered}
$$

donde, $\mathrm{p}_{\mathrm{nm}} \mathrm{y} \mathrm{p}_{\mathrm{nm}}^{\prime}$ son respectivamente los ceros de $\mathrm{J}_{\mathrm{n}}(\mathrm{x}) \mathrm{y} \mathrm{J}_{\mathrm{n}}^{\prime}(\mathrm{x})$, funciones de Bessel de 1 a especie y orden $n$ y sus derivadas.

\subsubsection{Principio Básico}

Cuando se origina una transición en la fase, se produce un cambio en la densidad respecto a la fase homogénea, esto provoca una discontinuidad en la constante dieléctrica de la nueva fase. La relación entre la densidad y la constante dieléctrica viene determinada como se comentó anteriormente mediante la ecuación de Clausius-Mossotti (ecuación 2.38). Para poder demostrar el principio de funcionamiento tanto cualitativo como cuantitativo en la cavidad para la determinación de las transiciones de fase, se recurre a los métodos de perturbaciones en cavidades resonantes empleados para el estudio de propiedades electromagnéticas de materiales. Birnbaum y Franeau [64], desarrollaron por primera en 1949 el método de perturbación en cavidades resonantes para la medida de la constante dieléctrica en sólidos y líquidos. Un sistema electromagnético, como es el caso de estudio en esta tesis doctoral, donde 
se utiliza una cavidad resonante en microondas con un tubo de zafiro en su eje central el cual contiene la muestra a estudio, puede caracterizarse por una serie de parámetros como las dimensiones del resonador, la conductividad de las paredes de este, la permitividad dieléctrica y la permeabilidad magnética del medio ocupado en el espacio de la cavidad.

La permitividad dieléctrica y la permeabilidad magnética, se calculan a partir de los cambios de la frecuencia de resonancia y del factor de calidad de la cavidad en el vacío y cuando contiene la muestra objeto de medida. Las transiciones de fases estudiadas en esta tesis doctoral (puntos de burbuja y de rocío) se pueden considerar como perturbaciones del medio dieléctrico, ya que la permitividad dieléctrica de la fase líquida y de la fase vapor pueden diferir de manera notable.

Considerando una cavidad resonante construida con un material conductor ideal [31], y encerrada en una superficie conocida $S$ con un volumen constante $V$, los campos eléctricos y magnéticos antes de la perturbación son $\mathbf{E}_{1} \mathrm{y}_{\mathbf{H}_{1}}$. De acuerdo con las ecuaciones de Maxwell se tiene:

$$
\begin{gathered}
\nabla \times \mathrm{E}_{1}=-\mathrm{j} \omega_{1} \mu_{1} \mathrm{H}_{1} \\
\nabla \times \mathrm{H}_{1}=\mathrm{j} \omega_{1} \varepsilon_{1} \mathrm{E}_{1}
\end{gathered}
$$

Donde $\omega_{1}$ es la frecuencia angular resonante de la cavidad anterior a la perturbación, $\varepsilon_{1}$ y $\mu_{1}$ son la permeabilidad y la permitividad del medio en la cavidad antes de la perturbación. Después de una pequeña perturbación, el campo eléctrico y el campo magnético serán $\mathbf{E}_{2}$ y $\mathbf{H}_{2}$. La pequeña perturbación puede ser un pequeño cambio en las propiedades de un volumen grande de material, como cuando la cavidad se llena con un gas cuya permitividad quiere ser medida, o un gran cambio de las propiedades de un material con un pequeño volumen como cuando un pequeño sólido se introduce en la cavidad. En esta tesis doctoral, esta 
perturbación es provocada por un cambio en la fase del fluido de la muestra, ya sea por la aparición de una burbuja de vapor cuando el sistema se encuentre en estado líquido (punto de burbuja) o una gota de líquido cuando este se encuentre en estado gaseoso (punto de rocío). En cualquiera de los casos las ecuaciones anteriores se transforman en las siguientes:

$$
\begin{gathered}
\nabla \times \mathrm{E}_{2}=-\mathrm{j} \omega_{2} \mu_{2} \mathrm{H}_{2} \\
\nabla \times \mathrm{H}_{2}=\mathrm{j} \omega_{2} \varepsilon_{2} \mathrm{E}_{2}
\end{gathered}
$$

Donde $\omega_{2}$ es la frecuencia angular resonante de la cavidad posterior a la perturbación, $\varepsilon_{2}$ y $\mu_{2}$ son la permeabilidad y la permitividad del medio en la cavidad después de la perturbación operando en las ecuaciones 3.5 y 3.6 se tiene.

$$
\begin{aligned}
& \mathrm{H}_{2} \cdot \nabla \times E_{1}^{*}=j \omega_{1} \mu_{1} \mathrm{H}_{2} \cdot H_{1}^{*} \\
& -E_{2} \cdot \nabla \times H_{1}^{*}=j \omega_{1} \mu_{1} E_{2} \cdot E_{1}^{*} \\
& H_{1}^{*} \cdot \nabla \times E_{2}=j \omega_{2} \mu_{2} H_{2} \cdot H_{1}^{*} \\
& -E_{1}^{*} \cdot \nabla \times H_{2}=j \omega_{2} \mu_{2} E_{2} \cdot E_{1}^{*}
\end{aligned}
$$

de acuerdo con el vector identidad.

$$
\mathrm{B} \cdot \nabla \times \mathrm{A}-\mathrm{A} \cdot \nabla \times \mathrm{B}=\nabla \cdot(\mathrm{A} \times \mathrm{B})
$$

Sumando las ecuaciones 3.7 a 3.10 se obtiene. 


$$
\begin{aligned}
\nabla \cdot\left(H_{2} \times E_{1}^{*}+\right. & \left.H_{1}^{*} \times E_{2}\right) \\
& =j \omega_{1} \mu_{1} H_{2} \cdot H_{1}^{*}+j \omega_{1} \mu_{1} E_{2} \cdot E_{1}^{*}+j \omega_{2} \mu_{2} H_{2} \cdot H_{1}^{*} \\
& +j \omega_{2} \mu_{2} E_{2} \cdot E_{1}^{*}
\end{aligned}
$$

que puede ser reescrita como:

$$
\begin{aligned}
\nabla \cdot\left(\mathrm{H}_{2} \times \mathrm{E}_{1}^{*}+\mathrm{H}_{1}^{*} \times \mathrm{E}_{2}\right) \\
\quad=\mathrm{j}\left[\left(\omega_{2} \varepsilon_{2}-\omega_{1} \varepsilon_{1}\right) \mathrm{E}_{2} \cdot \mathrm{E}_{1}^{*}+\left(\omega_{2} \mu_{2}-\omega_{1} \mu_{1}\right) \mathrm{H}_{2} \cdot \mathrm{H}_{1}^{*}\right]
\end{aligned}
$$

Integrando ambos lados de la igualdad de la ecuación 3.13 sobre el volumen V de la cavidad se obtiene que.

$$
\begin{aligned}
\int_{\mathrm{V}} \nabla \cdot\left(\mathrm{H}_{2} \times \mathrm{E}_{1}^{*}\right. & \left.+\mathrm{H}_{1}^{*} \times \mathrm{E}_{2}\right) \mathrm{dV} \\
& =\mathrm{j}\left[\left(\omega_{2}-\omega_{1}\right) \int_{\mathrm{V}}\left(\varepsilon_{1} \mathrm{E}_{2} \cdot \mathrm{E}_{1}^{*}+\mu_{1} \mathrm{H}_{2} \cdot \mathrm{H}_{1}^{*}\right) \mathrm{dV}\right. \\
& \left.+\omega_{2} \int_{\mathrm{V}}\left(\Delta_{\varepsilon} \mathrm{E}_{2} \cdot \mathrm{E}_{1}^{*}+\Delta_{\mu} \mathrm{H}_{2} \cdot \mathrm{H}_{1}^{*}\right) \mathrm{dV}\right]
\end{aligned}
$$

con.

$$
\begin{aligned}
& \Delta_{\varepsilon}=\varepsilon_{2}-\varepsilon_{1} \\
& \Delta_{\mu}=\mu_{2}-\mu_{1}
\end{aligned}
$$

Hasta ahora, no se ha realizado ninguna aproximación en la ecuación 3.14, siendo esta, la ecuación fundamental en la teoría de perturbaciones si la cavidad estuviera hecha por un material perfectamente conductor. Esta teoría puede ser aplicada a tres casos: perturbación por la forma de la cavidad, perturbación por 
impedancia de las paredes, y como es el caso en esta tesis doctoral, perturbación por introducción de materia.

En el límite de la superficie $S$ de una cavidad cuyo volumen es $V$, los campos eléctricos antes y después de la perturbación son iguales a cero; $\mathrm{n} \times \mathrm{E}_{1}^{*}=0 \mathrm{y}$ $\mathrm{n} \times \mathrm{E}_{2}=0$, y de acuerdo con el vector identidad.

$$
\int_{\mathrm{V}} \nabla \cdot(\mathrm{A} \times \mathrm{B}) \mathrm{dV}=\oint_{\mathrm{S}} \mathrm{A} \times \mathrm{B} \cdot \mathrm{dS}
$$

Se tiene que.

$$
\int_{V}\left(H_{2} \times E_{1}^{*}+H_{1}^{*} \times E_{2}\right) d V=0
$$

Por lo que la ecuación 3.14 puede ser escrita como.

$$
\frac{\Delta \omega}{\omega}=\frac{\omega_{2}-\omega_{1}}{\omega_{1}}=-\frac{\int_{\mathrm{V}_{\mathrm{c}}}\left(\Delta \varepsilon \mathrm{E}_{2} \cdot \mathrm{E}_{1}^{*}+\Delta \mu \mathrm{H}_{2} \cdot \mathrm{H}_{1}^{*}\right) \mathrm{dV}}{\int_{\mathrm{V}_{\mathrm{c}}}\left(\varepsilon_{1} \mathrm{E}_{2} \cdot \mathrm{E}_{1}^{*}+\mu_{1} \mathrm{H}_{2} \cdot \mathrm{H}_{1}^{*}\right) \mathrm{dV}}
$$

Donde $\omega_{1}$ y $\omega_{2}$ son las frecuencias resonantes angulares complejas antes y después de introducir la muestra, $\varepsilon_{1} y, \varepsilon_{2}$ son las permitividades complejas del medio original en la cavidad y de la muestra, $\mu_{1}$ y $\mu_{2}$ son las permeabilidades complejas magnéticas del medio original en la cavidad y de la muestra $\mathbf{H}_{1}, \mathbf{H}_{2}$ y $\mathbf{E}_{1}$, $\mathbf{E}_{2}$ son los campos magnéticos y campos eléctricos respectivamente antes $\mathbf{y}$ después de la introducción la muestra y $V_{c}$ es la región encerrada por la cavidad. Esta última ecuación asume que la cavidad es perfectamente conductora y que la perturbación originada es pequeña, cuando se considera que la muestra es homogénea, que ocupa una pequeña porción de la cavidad y que el campo 
electromagnético no varía al introducir la muestra, la ecuación 3.19 puede reescribirse de la siguiente manera.

$$
\frac{\omega_{2}-\omega_{1}}{\omega_{1}} \approx-\frac{\int_{\mathrm{V}_{\mathrm{s}}}\left(\Delta \varepsilon \mathrm{E}_{2} \cdot \mathrm{E}_{1}^{*}+\Delta \mu \mathrm{H}_{2} \cdot \mathrm{H}_{1}^{*}\right) \mathrm{dV}}{2 \int_{\mathrm{V}_{\mathrm{c}}} \varepsilon_{1} \mathrm{E}_{2} \cdot \mathrm{E}_{1}^{*} \mathrm{dV}}
$$

Donde $\mathrm{V}_{\mathrm{s}}$ corresponde al volumen de la muestra introducida en la cavidad.

Cuando la muestra introducida posee pérdidas dieléctricas, es necesario realizar una serie de modificaciones que puedan satisfacer los cálculos para las medias de la permitividad y la permeabilidad de la muestra introducida. Para ello, se asume que se le ha realizado el vacío en el interior de la cavidad que contiene la muestra, de modo que $\mu_{1}=\mu_{2}=\mu_{0}$ y $\varepsilon_{1}=\varepsilon_{0}$, por lo que la ecuación 3.20 quedaría expresada como,

$$
\frac{\omega_{2}-\omega_{1}}{\omega_{1}}=-\left(\frac{\varepsilon_{\mathrm{r}}-1}{2}\right) \frac{\iiint_{\mathrm{V}_{\mathrm{s}}} \mathrm{E}_{1} \cdot \mathrm{E}_{2} \mathrm{dV}}{\iiint_{\mathrm{V}_{\mathrm{c}}}\left|\mathrm{E}_{1}\right|^{2} \mathrm{dV}}
$$

donde $\varepsilon_{\mathrm{r}}$ es la permitividad compleja relativa de la muestra: $\varepsilon_{\mathrm{r}}=\varepsilon_{2} / \varepsilon_{0}$.

La frecuencia angular compleja $\omega$ de la cavidad resonante está relacionada con la frecuencia $f$ y el factor de calidad $Q$ de la cavidad.

$$
\begin{gathered}
\omega=\omega_{\text {real }}+j \omega_{\text {imag. }} \\
\omega_{\text {real }}=2 \pi f \\
Q=\frac{\omega_{\text {real }}}{2 \omega_{\text {imag. }}}
\end{gathered}
$$


Si se considera $\omega_{\text {real1 }} \approx \omega_{\text {real2 }}$ y $\omega_{\text {imag. }} \ll \omega_{\text {real }}$, entonces se obtiene:

$$
\begin{gathered}
\frac{\omega_{2}-\omega_{1}}{\omega_{1}}=-\frac{\left(\omega_{\text {real2 }}-\omega_{\text {real1 }}\right)+j\left(\omega_{\text {imag.2 }}-\omega_{\text {imag.1 }}\right)}{\omega_{\text {real2 }}\left(1+j \frac{\omega_{\text {imag.2 }}}{\omega_{\text {real2 }}}\right)} \\
\approx\left[\left(\frac{f_{2}-f_{1}}{f_{2}}\right)+j\left(\frac{1}{2 Q_{2}}-\frac{1}{2 Q_{1}}\right)\right]\left(1-j \frac{1}{2 Q_{2}}\right) \\
\approx\left(\frac{f_{2}-f_{1}}{f_{2}}\right)+j\left(\frac{1}{2 Q_{2}}-\frac{1}{2 Q_{1}}\right)
\end{gathered}
$$

considerando $Q_{2} \gg 1$, de la ecuación 3.25 se tiene.

$$
\left(\frac{\mathrm{f}_{2}-\mathrm{f}_{1}}{\mathrm{f}_{2}}\right)+\mathrm{j}\left(\frac{1}{2 \mathrm{Q}_{2}}-\frac{1}{2 \mathrm{Q}_{1}}\right)=-\left(\frac{\varepsilon_{\mathrm{r}}-1}{2}\right) \frac{\iiint_{\mathrm{V}_{\mathrm{s}}} \mathrm{E}_{1} \cdot \mathrm{E}_{2} \mathrm{dV}}{\iiint_{\mathrm{V}_{\mathrm{c}}}\left|\mathrm{E}_{1}\right|^{2} \mathrm{dV}}
$$

La ecuación 3.26 puede ser escrita como,

$$
\begin{gathered}
2\left(\frac{\mathrm{f}_{2}-\mathrm{f}_{1}}{\mathrm{f}_{2}}\right)=\left(\varepsilon_{\mathrm{r}}^{\prime}-1\right) \mathrm{C} \\
\frac{1}{\mathrm{Q}_{2}}-\frac{1}{\mathrm{Q}_{1}}=\varepsilon_{\mathrm{r}}^{\prime \prime} \mathrm{C}
\end{gathered}
$$

donde.

$$
C=\frac{\iiint_{V_{s}} E_{1} \cdot E_{2} d V}{\iiint_{V_{c}}\left|E_{1}\right|^{2} d V}
$$


En el método de perturbación de cavidades, el parámetro C se supone constante e independiente de las propiedades de la muestra. Sin embargo, en la ecuación 3.29, el campo perturbado en la muestra $E_{2}$ está relacionado con la permitividad, forma y tamaño de la muestra bajo estudio, esto origina que el parámetro C cambie caso a caso. Por lo tanto, en un sentido estricto, las ecuaciones 3.28 y 3.29 muestran que el cambio en el factor de calidad debido a la perturbación no solo está relacionado con la parte imaginaria de la permitividad de la muestra, sino que también depende de la parte real de la permitividad de la muestra. Para hacer más clara la expresión anterior se introducen los parámetros A y B.

$$
\begin{gathered}
\frac{\mathrm{f}_{1}-\mathrm{f}_{2}}{\mathrm{f}_{2}}=\mathbf{A} \frac{\mathrm{V}_{\mathrm{s}}}{\mathrm{V}_{\mathrm{c}}}\left(\varepsilon_{\mathrm{r}}^{\prime}-1\right) \\
\frac{1}{\mathrm{Q}_{2}}-\frac{1}{\mathrm{Q}_{1}}=\mathbf{B} \frac{\mathrm{V}_{\mathrm{s}}}{\mathrm{V}_{\mathrm{c}}} \varepsilon_{\mathrm{r}}^{\prime \prime}
\end{gathered}
$$

Los parámetros $A$ y $B$, en forma similar al parámetro $C$, están relacionados con la configuración y el modo de funcionamiento de la cavidad, la forma de la muestra y su localización. Es muy difícil calcular analíticamente estos parámetros, y se obtienen mediante calibración de muestras estándar de permitividad o constante dieléctrica conocida.

\subsubsection{Cavidad cilíndrica con tubo de muestreo}

En el caso general del método de las perturbaciones descrito en el apartado anterior, se parte del supuesto de una cavidad en vacío cuya muestra está contenida en su interior, en esta tesis doctoral, la cavidad desarrollada tiene un tubo de zafiro en su eje longitudinal que contiene la pequeña porción de la muestra bajo estudio. Esto origina que la ecuación 3.21 quede de la siguiente forma. 


$$
\frac{\omega_{2}-\omega_{1}}{\omega_{1}}=-\left(\frac{\varepsilon_{\mathrm{r} 2}-\varepsilon_{\mathrm{r} 1}}{2 \varepsilon_{\mathrm{r} 1}}\right) \frac{\iiint_{\mathrm{V}_{\mathrm{s}}} \mathrm{E}_{1} \cdot \mathrm{E}_{2} \mathrm{dV}}{\iiint_{\mathrm{V}_{\mathrm{c}}}\left|\mathrm{E}_{1}\right|^{2} \mathrm{dV}}
$$

De igual manera la ecuación 3.26 se transforma en.

$$
\left(\frac{\mathrm{f}_{2}-\mathrm{f}_{1}}{\mathrm{f}_{2}}\right)+\mathrm{j}\left(\frac{1}{2 \mathrm{Q}_{2}}-\frac{1}{2 \mathrm{Q}_{1}}\right)=-\left(\frac{\varepsilon_{\mathrm{r} 2}-\varepsilon_{\mathrm{r} 1}}{2 \varepsilon_{\mathrm{r} 1}}\right) \frac{\iiint_{\mathrm{V}_{\mathrm{s}}} \mathrm{E}_{1} \cdot \mathrm{E}_{2} \mathrm{dV}}{\iiint_{\mathrm{V}_{\mathrm{c}}}\left|\mathrm{E}_{1}\right|^{2} \mathrm{dV}}
$$

Considerando despreciables las pérdidas dieléctricas del tubo que contiene la muestra, y que el fluido es no polar, teniendo en cuenta únicamente el término real de la ecuación 3.33, la ecuación 3.27 queda de la siguiente forma:

$$
2\left(\frac{\mathrm{f}_{1}-\mathrm{f}_{2}}{\mathrm{f}_{2}}\right)=\left(\frac{\varepsilon_{\mathrm{r} 2}^{\prime}-\varepsilon_{\mathrm{r} 1}^{\prime}}{2 \varepsilon_{\mathrm{r} 1}^{\prime}}\right) \mathrm{C}
$$

Dado que el volumen de la muestra en el interior de tubo y de la cavidad son constantes, la ecuación 3.34 se puede particularizar para el caso que ocupa esta tesis doctoral de una cavidad cilíndrica en microondas con tubo de muestreo en su eje longitudinal y que contiene una muestra en fase homogénea.

$$
\frac{\mathrm{f}_{1}-\mathrm{f}_{2}}{\mathrm{f}_{2}}=\mathbf{A} \frac{\mathrm{V}_{\mathrm{s}}}{\mathrm{V}_{\mathrm{c}}}\left(\frac{\varepsilon_{\mathrm{r} 2}^{\prime}-\varepsilon_{\mathrm{r} 1}^{\prime}}{\varepsilon_{\mathrm{r} 1}^{\prime}}\right)
$$

La discontinuidad de la constante dieléctrica debido a la aparición de una nueva fase se verá reflejada como una variación en la frecuencia de resonancia de la cavidad (ecuación 3.35). De igual manera, el cambio de presión en la aparición de una nueva fase a temperatura constante hace que la frecuencia de resonancia 
varíe debido al cambio en la densidad de la muestra. Esta variación es continua y lineal, por lo que se puede normalizar mediante un polinomio de segundo grado, descartando en la frecuencia, el efecto de la presión sobre la densidad de la fase quedando únicamente la discontinuidad provocada por la transición de la fase en la aparición de un punto de burbuja o de rocío.

$$
f(p)=a_{2} p^{2}+a_{1} p+a_{0}
$$

Realizado una representación de $\left(\mathrm{f}_{0}-\mathrm{a}_{2} \mathrm{p}^{2}-\mathrm{a}_{1} \mathrm{p}\right) / \mathrm{a}_{0}$ frente a la presión, y se ha conseguido normalizar la influencia de la presión en la frecuencia de resonancia. La función normalizada permite obtener la discontinuidad en la frecuencia de resonancia en la aparición de los puntos de rocío y de burbuja.

\subsubsection{Modo resonante $\mathrm{TM}_{010}$}

Las cavidades cilíndricas usualmente operan en modos resonantes del tipo TM (transversal magnético) estos incluyen dos categorías: los modos $\mathrm{TM}_{0 \mathrm{no}}$ y los modos $\mathrm{TM}_{01 \mathrm{n}}[31]$ en esta tesis doctoral nos centraremos en los modos $\mathrm{TM}_{0 \mathrm{no}}$, $\mathrm{Y}$ en particular el modo resonante TM010. En la figura 3.13, se muestra la distribución de los campos eléctricos y magnéticos, en la que distribución del campo magnético es paralelo a la superficie y perpendicular al campo eléctrico. La densidad del campo magnético se va incrementando desde el eje central de la cavidad hacia la superficie que la delimita, mientras que para el campo eléctrico sucede, al contrario, es más intenso en el eje de la cavidad. 


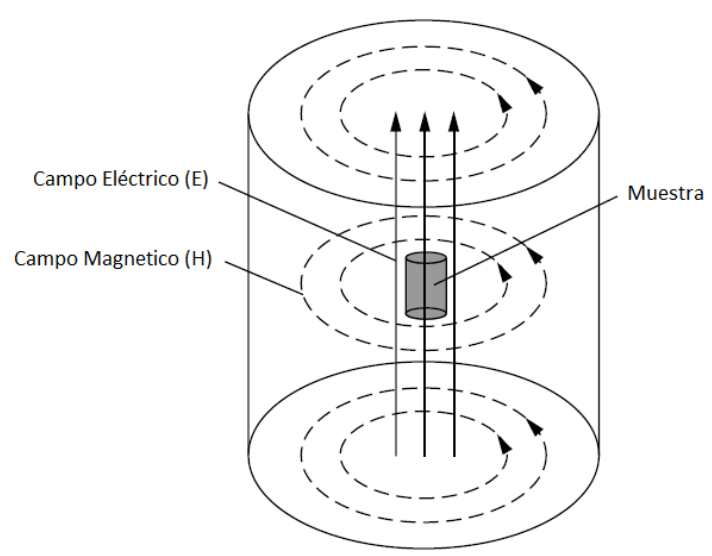

Figura 3.13 Distribución de los campos eléctricos $(E)$ y magnéticos $(H)$ de la cavidad cilíndrica resonante.

Las componentes en coordenadas cilíndricas del campo electromagnético [65] para el modo de $\mathrm{TM}_{010}$ son las siguientes:

$$
\begin{gathered}
\overline{\mathrm{E}}_{\mathrm{r}}=-\mathrm{j} \beta \mathrm{k}_{\mathrm{c}} \overline{\mathrm{B}} \mathrm{J}_{\mathrm{n}}^{\prime}\left(\mathrm{k}_{\mathrm{c}} \mathrm{r}\right) \cos \mathrm{n} \phi \mathrm{e}^{-\mathrm{j} \beta \mathrm{z}} \\
\overline{\mathrm{E}}_{\phi}=\mathrm{j} \beta \frac{\mathrm{n}}{\mathrm{r}} \overline{\mathrm{B}} \mathrm{J}_{\mathrm{n}}\left(\mathrm{k}_{\mathrm{c}} \mathrm{r}\right) \operatorname{sen} \mathrm{n} \phi \mathrm{e}^{-\mathrm{j} \beta \mathrm{z}} \\
\overline{\mathrm{E}}_{\mathrm{z}}=\mathrm{k}^{2} \overline{\mathrm{B}} \mathrm{J}_{\mathrm{n}}\left(\mathrm{k}_{\mathrm{c}} \mathrm{r}\right) \cos \mathrm{n} \phi \mathrm{e}^{-\mathrm{j} \beta \mathrm{z}} \\
\overline{\mathrm{H}}_{\mathrm{r}}=-\mathrm{j} \omega \varepsilon_{0} \frac{\mathrm{n}}{\mathrm{r}} \overline{\mathrm{B}} \mathrm{J}_{\mathrm{n}}\left(\mathrm{k}_{\mathrm{c}} \mathrm{r}\right) \operatorname{sen} \mathrm{n} \phi \mathrm{e}^{-\mathrm{j} \beta \mathrm{z}} \\
\overline{\mathrm{H}}_{\phi}=-\mathrm{j} \omega \varepsilon_{0} \mathrm{k}_{\mathrm{c}} \overline{\mathrm{B}} \mathrm{J}_{\mathrm{n}}^{\prime}\left(\mathrm{k}_{\mathrm{c}} \mathrm{r}\right) \cos \mathrm{n} \phi \mathrm{e}^{-\mathrm{j} \beta \mathrm{z}} \\
\overline{\mathrm{H}}_{\mathrm{z}}=0
\end{gathered}
$$

Donde $\mathrm{j}$ define el número complejo, $\beta$ representa el coeficiente de fase $\mathrm{y} \mathrm{k}_{\mathrm{c}}=$ $\beta_{0}^{2}-\beta^{2}$ es un parámetro dependiente de este coeficiente $\beta, \overline{\mathrm{B}}$ es el flujo de densidad magnético, $\mathrm{J}_{\mathrm{n}}^{\prime}$ es la función de Besel de primer orden y $\phi$ es el potencial normalizado. 
La frecuencia de resonancia en el modo $\mathrm{TM}_{010}$ en concreto viene determinada por la ecuación 3.2, siendo el cero de la ecuación de Bessel $\mathrm{p}_{01}=2.405$ por lo que la frecuencia de resonancia se expresa como:

$$
\mathrm{f}_{010}=\frac{\mathrm{c}_{0}}{2 \pi \sqrt{\varepsilon_{\mathrm{r}} \mu_{\mathrm{r}}}}\left(\frac{2.405}{\underline{\mathrm{a}}}\right)
$$

Que va a depender exclusivamente del radio de la cavidad a las propiedades electromagnéticas $\varepsilon_{\mathrm{r}} \mu_{\mathrm{r}} \cdot \mathrm{y}$ la velocidad de la luz en el vacío $c_{0}$.

EL factor de calidad (Q) es un parámetro esencial para estimar la calidad de la cavidad resonante [63]. Valores elevados de este parámetro indican un ancho de banda estrecho y una precisión en la cavidad alta. El factor de calidad de la resonancia se descompone en dos términos, la aportación de la cavidad debido a la conductividad de su superficie $\left(Q_{c}\right)$ y la aportación del dieléctrico que se encuentra en el volumen de la cavidad $\left(Q_{d}\right)$.

$$
\frac{1}{\mathrm{Q}}=\frac{1}{\mathrm{Q}_{\mathrm{c}}}+\frac{1}{\mathrm{Q}_{\mathrm{d}}}
$$

El modo $\mathrm{TM}_{010}$ es adecuado para fluidos no polares, como los componentes que se han empleado en las medidas, ya que presentan valores bajos de constantes dieléctricas y bajas pérdidas dieléctricas. Para fluidos polares con altas pérdidas dieléctricas y valores altos de constante dieléctrica, el modo $\mathrm{TM}_{010}$, donde la muestra se sitúa en el eje, lleva a que la frecuencia de resonancia cambie bruscamente y el factor de calidad disminuya considerablemente. Gregory y Clarke [32] consideran el modo $\mathrm{TE}_{011}$ más adecuado para los fluidos polares puesto que la distribución del campo eléctrico de este modo es radial en planos perpendiculares al eje. 


\subsubsection{Medida del factor de calidad y frecuencia de resonancia}

La determinación de manera precisa tanto del factor de calidad Q como de la frecuencia de resonancia $f_{0}$ de un resonador en microondas, empleando para ello los datos obtenidos del coeficiente complejo de transmisión $\overrightarrow{\mathrm{S}_{21}}(\mathrm{f})$ como función de la frecuencia, se vuelve complejo cuando se estudian en condiciones realistas de operación como es el caso de esta tesis doctoral. En el funcionamiento del medidor hay que tener en cuenta los efectos de "crosstalk" entre las líneas de transmisión y las estructuras de los acoplamientos, la separación entre los puertos de acoplamientos y el instrumento de medida, y el ruido que pueda originar el equipo que transmite la onda. Petersan y Anlage [66], realizaron una comparación con siete métodos diferentes de ajuste empleando para ello el coeficiente complejo de transmisión frente a la frecuencia, realizando además una valoración de estos métodos en presencia de ruido. Para esta tesis doctoral se ha empleado el ajuste de los datos del módulo complejo de transmisión frente a la frecuencia, ajustando los datos experimentales a una curva lorenziana, siendo este el método más preciso para los datos con alto contenido en ruido.

Un resonador puede ser representado por una serie de circuitos RLC [31], y si el plano de referencia es elegido en el plano donde la integración del campo magnético es máxima, el resonador puede ser representado por un circuito paralelo equivalente RLC, cuya frecuencia de resonancia vendrá determinada por la relación $\mathrm{f}_{0}=1 / \sqrt{\mathrm{LC}}$, y factor de calidad definido como la energía total almacenada en el resonador y la energía disipada por el ciclo determinado mediante la relación $\mathrm{Q}=\mathrm{f}_{0} \mathrm{RC}$ [63].

Para la estimación del factor de calidad y la frecuencia de resonancia se emplea el modelo que Coakley et al. [67] desarrollaron para un resonador cilíndrico acoplado a dos puertos. El circuito equivalente RLC con las dos líneas de transmisión y la cavidad acoplada, se muestra en la figura 3.14. 


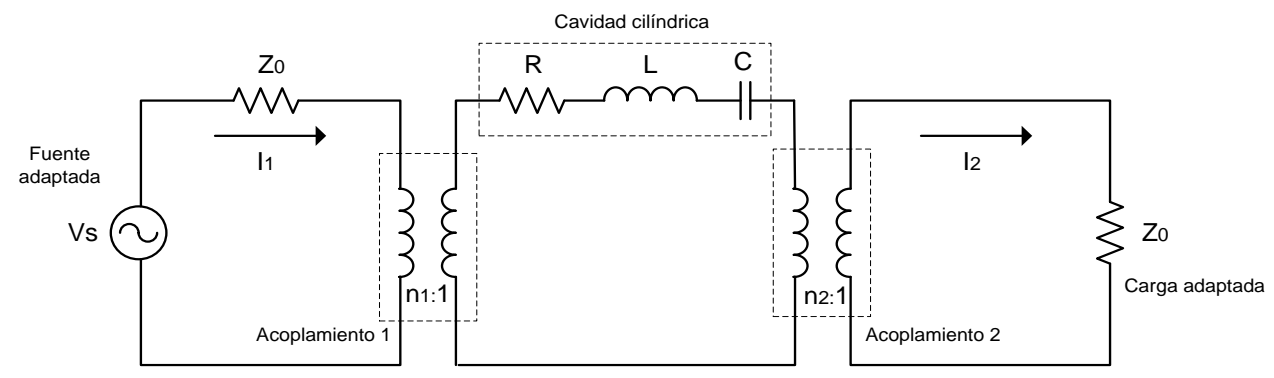

Figura 3.14 Circuito eléctrico equivalente con cavidad acoplada.

En particular nos interesan las medidas con una cavidad desacoplada, figura 3.15, con un alto factor de calidad operando cerca de la resonancia, en este caso, asumimos que las resistencias y auto inductancias de los circuitos de los acoplamientos son despreciables.

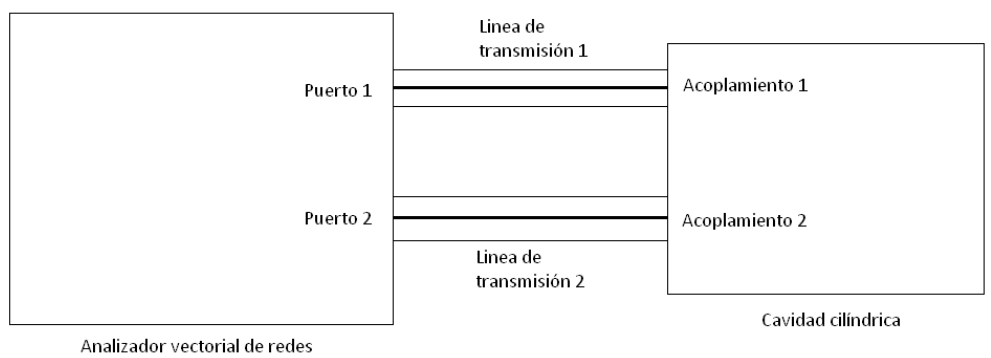

Figura 3.15 Diagrama de bloques con la cavidad desacoplada.

Una fuente de impedancia coincidente es conectada al puerto uno de la cavidad, mientras que otra fuente con carga adaptada es conectada al puerto dos, las fuentes pueden ser intercambiadas sin originar con ello pérdidas en la transmisión. Definimos pues las pérdidas de transmisión T(f) a través de la cavidad cilíndrica como:

$$
T(f)=\frac{P_{\text {in }}}{P_{L}}
$$


Donde fes la frecuencia, $\mathrm{P}_{\text {in }}$ es la máxima potencia suministrada a una carga adaptada conectada al puerto uno, y $\mathrm{P}_{\mathrm{L}}$ es la máxima potencia suministrada a una carga adaptada al puerto dos. Resolviendo los valores de $\mathrm{P}_{\text {in }}$ y $\mathrm{P}_{\mathrm{L}}$ mediante la teoría de circuitos se tiene,

$$
\begin{gathered}
P_{\text {in }}=I_{1} I_{1}^{*} Z_{0}=\frac{V_{s}^{2}}{4 Z_{0}} \\
P_{L}=I_{2} I_{2}^{*} Z_{0}=\frac{V_{s}^{2}}{4 Z_{0}} \frac{\beta_{1} \beta_{2}}{\left(1+\beta_{1}+\beta_{2}\right)^{2}+Q_{0}^{2}\left(\frac{f}{f_{0}}-\frac{f_{0}}{f}\right)^{2}}
\end{gathered}
$$

donde.

$$
\begin{aligned}
& \beta_{1}=\frac{\mathrm{n}_{1}^{2} \mathrm{Z}_{0}}{\mathrm{R}} \\
& \beta_{2}=\frac{\mathrm{n}_{2}^{2} \mathrm{Z}_{0}}{\mathrm{R}}
\end{aligned}
$$

En la ecuación 3.47 la frecuencia $\mathrm{f}_{0}$ se define como,

$$
f_{0}=\frac{1}{4 \pi^{2} L C}
$$

y el factor de calidad en el vacío $Q_{0}$ será.

$$
\mathrm{Q}_{0}=\frac{2 \pi \mathrm{f}_{0} \mathrm{~L}}{\mathrm{R}}
$$

Substituyendo las ecuaciones 3.46 y 3.47 en la ecuación 3.45. 


$$
T(f)=\frac{4 \beta_{1} \beta_{2}}{\left(1+\beta_{1}+\beta_{2}\right)^{2}+Q_{0}^{2}\left(\frac{f}{f_{0}}-\frac{f_{0}}{f}\right)^{2}}
$$

Cuando la resonancia $\mathrm{f}=\mathrm{f}_{0}$ la pérdida de transmisión se reduce a:

$$
\mathrm{T}\left(\mathrm{f}_{0}\right)=\frac{4 \beta_{1} \beta_{2}}{\left(1+\beta_{1}+\beta_{2}\right)^{2}}
$$

Tomando la proporción $\mathrm{T}\left(\mathrm{f}_{0}\right) / \mathrm{T}(\mathrm{f})$ se obtiene:

$$
\frac{T\left(f_{0}\right)}{T(f)}=1+\frac{Q_{0}^{2}\left(\frac{f}{f_{0}}-\frac{f_{0}}{f}\right)^{2}}{\left(1+\beta_{1}+\beta_{2}\right)^{2}}
$$

Cabe destacar que, en la práctica, el factor de calidad con la cavidad en vacío es mucho mayor que el obtenido cuando la cavidad contiene un medio, esto es debido a los efectos de bucles de acoplamiento.

$$
Q_{0}=Q\left(1+\beta_{1}+\beta_{2}\right)
$$

Sin embargo, si se reduce el nivel de acoplamiento hasta un nivel que se considera desacoplada $\beta_{1} \ll 1$ y $\beta_{2} \ll 1$, podríamos considerar los factores $\beta_{1}$ y $\beta_{2}$ como despreciables, reescribiendo la ecuación 3.54 de la siguiente manera:

$$
T(f)=\frac{T\left(f_{0}\right)}{1+Q^{2}\left(\frac{f}{f_{0}}-\frac{f_{0}}{f}\right)^{2}}
$$

Teniendo en cuenta que $T(f)=\left|S_{21}(f)\right|^{2}$ y asumiendo que $f \sim f_{0}[66]$ cerca de la resonancia, se simplifica la dependencia de la frecuencia en el denominador resultando la ecuación 3.56 de la siguiente manera. 


$$
\left|S_{21}(f)\right|^{2}=\frac{\left|\overline{S_{21}}\right|^{2}}{1+4 Q^{2}\left(\frac{f}{f_{0}}-1\right)^{2}}
$$

Donde $\overline{\mathrm{S}_{21}}$ es el coeficiente máximo de transmisión que puede ocurrir en el pico de la resonancia. Luego, el módulo del coeficiente complejo de transmisión estaría definido por la ecuación 3.58 .

$$
\left|S_{21}(f)\right|=\frac{\left|\overline{S_{21}}\right|}{\sqrt{1+4 Q^{2}\left(\frac{f}{f_{0}}-1\right)^{2}}}
$$

La gráfica del módulo del coeficiente complejo de transmisión frente a la frecuencia forma una curva de tipo lorenziana, con la frecuencia resonante localizada en la posición de su magnitud máxima figura 3.16. Las técnicas convencionales de ajuste no permiten el ajuste de los datos experimentales de manera directa mediante la ecuación 3.58, de acuerdo con Martin et al. [68], la ecuación 3.57 puede ser reescrita de la siguiente manera:

$$
\frac{1}{\left|S_{21}(f)\right|^{2}}=A f^{2}+B f+C
$$

Siendo estos coeficientes A, B y C los siguientes:

$$
\begin{aligned}
& A=\frac{4 Q^{2}}{\left|\overline{S_{21}}\right|^{2} f_{0}^{2}} \\
& B=\frac{-8 Q^{2}}{\left|\overline{S_{21}}\right|^{2} f_{0}}
\end{aligned}
$$




$$
C=\frac{1+4 Q^{2}}{\left|\overline{S_{21}}\right|^{2}}
$$

Donde los coeficientes del polinomio de segundo grado obtenido se pueden obtener de manera muy simple mediante un ajuste por mínimos cuadrados.

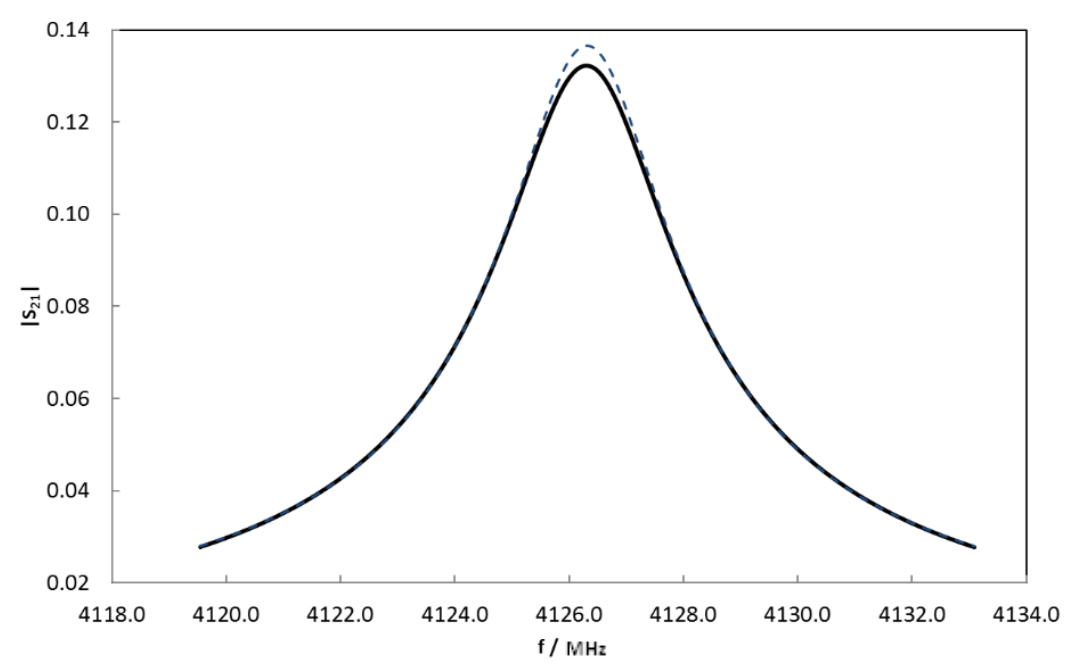

Figura 3.16 Representación lorenziana del coeficiente de transmisión complejo $\left|\mathrm{S}_{21}\right|$ frente a la frecuencia f datos experimentales (- ) curva ajustada (-).

Resolviendo las ecuaciones anteriores se obtiene que.

$$
\begin{gathered}
f_{0}=\frac{-B}{2 C} \\
Q=\sqrt{\frac{-B f_{0}}{4 B f_{0}+8 A}} \\
\left|\overline{S_{21}}\right|=\sqrt{\frac{1+4 Q^{2}}{A}}
\end{gathered}
$$


Este método permite obtener el valor de la frecuencia de resonancia y el factor de calidad de manera muy precisa, sin embargo, determinar los valores de $f_{0}$ y $Q$ requieren el uso del término independiente $C$, el cual en comparación con los valores de los otros dos coeficientes presenta errores significativos, por lo que una posible modificación a la ecuación 3.57 puede ser resuelta de la manera siguiente,

$$
\frac{1}{\left|S_{21}(f)\right|^{2}}=y=M+N x
$$

donde.

$$
\mathrm{x}=\left(\mathrm{f} / \mathrm{f}_{0}-1\right)^{2}
$$

Luego una simple regresión lineal de primer orden nos permite obtener los valores de $f_{0}$ y $Q$, siendo los parámetros $M$ y $N$ los siguientes:

$$
\begin{aligned}
& M=\frac{1}{\left|\overline{S_{21}}\right|^{2}} \\
& N=\frac{4 Q^{2}}{\left|\overline{S_{21}}\right|^{2}}
\end{aligned}
$$



CAPítulo 4

\section{Equipo experimental}





\subsection{Introducción}

Numerosos son los elementos que conforman un equipo experimental, de igual manera, la comunicación entre todos los elementos mediante los programas necesarios es importante, así como el control de manera automática para la toma y adquisición de datos experimentales de todos los elementos que conforman el equipo. En este capítulo, se describirá el equipo principal que ha sido empleado para la determinación del equilibrio de fases realizado en esta tesis doctoral. Hasta ahora en los capítulos anteriores se ha venido desarrollando el marco teórico de las cavidades resonantes en microondas mediante el método de las perturbaciones, con una detallada revisión bibliográfica de las técnicas experimentales para la medida de la constante dieléctrica de fluidos. En el capítulo que se presenta a continuación se describirán con detalle los aspectos técnicos y constructivos de la celda de medida, la cual, junto con el analizador vectorial de redes (VNA), son las piezas más importantes para el desarrollo de esta tesis doctoral. Además, se detallarán los aspectos técnicos de los equipos auxiliares de los que se compone el equipo experimental y el software empleado para el control automático y adquisición de datos de todos los elementos del equipo.

El aparato descrito en esta sección consiste en un trabajo original diseñado en el laboratorio de TERMOCAL (termodinámica y calibración) de la Universidad de Valladolid, y construido por DG-Technology, empresa ubicada en Parma (Italia), dedicada a la construcción de equipos de alta precisión mediante tecnología de control numérico computarizado (CNC).

\subsection{Descripción de la cavidad cilíndrica resonante en microondas}

En general, la cavidad de microondas es un recinto metálico, que admite una oscilación electromagnética formada por el cortocircuito de dos extremos de una 
guía de onda. La energía electromagnética se almacena dentro del recinto de la cavidad y la energía se disipa en la pared metálica y el material dieléctrico dentro de la cavidad [63]. A medida que las ondas eléctricas y magnéticas se reflejan en los límites axiales, se constituyen patrones de ondas estacionarias que forman los modos resonantes de la cavidad. Los modos se definen como un patrón de movimiento que se repite sinusoidalmente [69]. Con el fin de lograr el acoplamiento de la cavidad a una fuente externa, con el propósito de excitar un modo en particular, se puede emplear una sonda o probeta.

El diseño de la cavidad que ocupa esta tesis doctoral se basa en el concepto descrito por Ángel Gómez [15] al que se le han realizado una serie de mejoras, tanto en diseño como en funcionalidad. En la figura 4.1 se muestra una vista de sección de la cavidad cilíndrica empleada en esta tesis doctoral, la cavidad principal está construida en una aleación de cobre-zirconio al 15\% (ZrK015) suministrada por Luvata ${ }^{\circledast}$, con el fin de evitar la posible corrosión en cavidades de cobre, un acabado en la cara interior de la cavidad tipo "espejo" proporciona una mejora en el factor de calidad de los modos resonantes. La cavidad tiene un diámetro interior de $50 \mathrm{~mm}$ y una altura de $50 \mathrm{~mm}$, con un volumen total aproximado de $98 \mathrm{~cm}^{3}$. La cavidad está delimitada por tres piezas; el cuerpo cilíndrico principal y dos tapas que se ajustan al cuerpo principal mediante ocho barras roscadas M6 que encierran por completo la cavidad, y una junta tórica Kalrez ${ }^{\circledR}$ que proporciona un sello hermético en la cavidad. Con el fin de asegurar un buen ajuste en el montaje entre las tapas y la cavidad, se han dispuesto unos pasadores cilíndricos Goupilles Clindriques ISO2338.

Para realizar el vacío en el interior de la cavidad, en la tapa inferior, se dispone de un orificio desde el cual se acopla una bomba de vacío rotativa tipo Oerlikon TRIVAC D8B. El centro de las tapas de la cavidad esta agujereado de tal manera que se puede alojar el tubo de zafiro que contendrá la muestra, estos orificios están 
provistos de un rebaje que permite alojar una junta tórica de material Kalrez ${ }^{\circledR}$ descrita más arriba y permiten mantener el vacío en el interior de la cavidad.

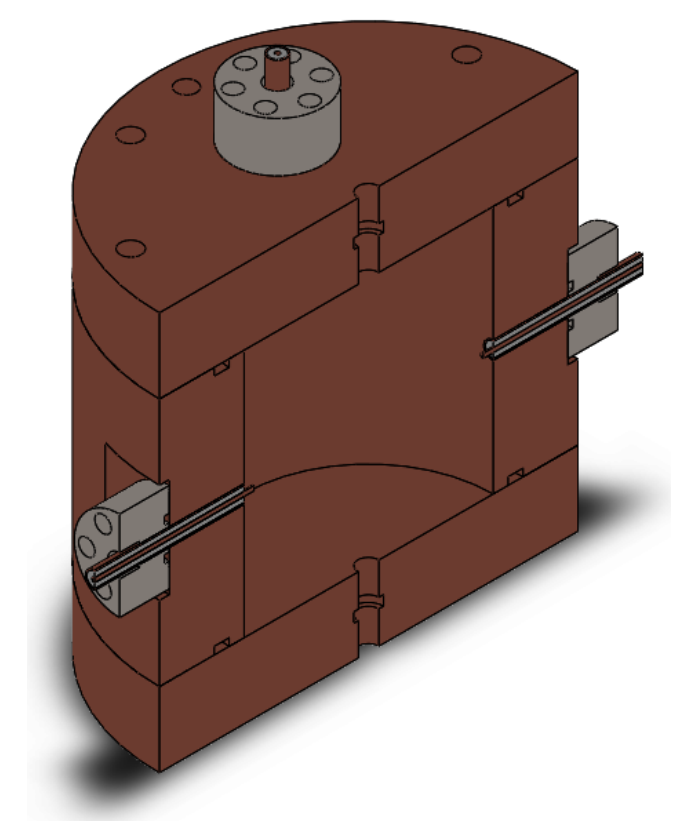

Figura 4.1 Vista de sección de la cavidad cilíndrica en microondas. 
El equipo desarrollado para esta tesis doctoral puede trabajar en los modos de resonancia TM y TE, para ello cuatro acoplamientos eléctricos se ubican en los ejes transversal y longitudinal de la cavidad, los soportes de estos acoplamientos están construidos en acero inoxidable 316, seis tornillos allen M4 lo atornillan al equipo y una junta tórica Kalrez ${ }^{\circledR}$ crea el sello necesario para mantener el vacío en la cavidad. Se utiliza un cable SMA coaxial semirrígido cuyo conductor interior es de plata recubierto en cobre de $0.92 \pm 0.02 \mathrm{~mm}$ de diámetro y capaz de trabajar a 20 $\mathrm{GHz}$ con una impedancia máxima de $50 \pm 1 \Omega$, que transmitirá la señal de microondas. La figura 4.2 muestra una sección transversal del sistema de acoplamiento del cable coaxial empleado. En el extremo de cada cable coaxial, se ha soldado un conector coaxial SMA C Straight Plug, Solder de $50 \Omega$, que servirá de acoplamiento con el analizador vectorial de redes.

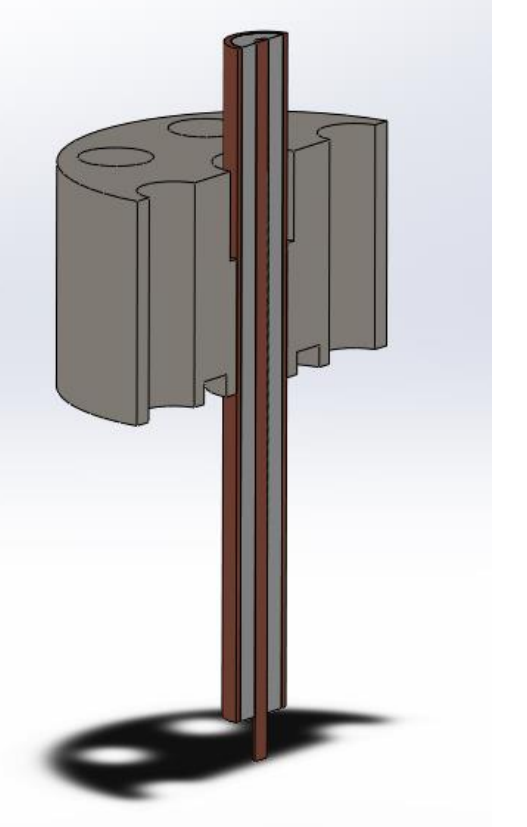

Figura 4.2 Vista de sección del soporte del cable coaxial. 
Como se comentó anteriormente, el material del tubo empleado para contener la muestra de estudio ha sido el zafiro, suministrado por Crystals Saint-Gobain. Se ha optado por emplear este material, dado que se trata de un material anisotrópico por lo que posee una muy baja pérdida dieléctrica en la dirección del eje que son las que más afectan al modo resonante. En cuanto a sus propiedades físicas, el zafiro nos permite trabajar a elevadas presiones teniendo en cuenta su resistencia a la compresión y su módulo de tracción; para determinar la presión máxima teórica a la que podemos someter el tubo empleado, es necesario conocer la geometría de este. Para esta tesis doctoral el tubo empleado tiene una longitud de $150 \mathrm{~mm}$, un diámetro exterior de $3.99 \mathrm{~mm}$, e interior de $2.01 \mathrm{~mm}$ con un volumen interno según esta geometría de $0.48 \mathrm{~cm}^{3}$. Acorde estas características podremos emplear la ecuación de Barlow [70] para determinar la presión teórica máxima, siendo para este caso de $200 \mathrm{MPa}$. Para soportar esta presión, se ha desarrollado un sistema de sujeción que se muestra en la figura 4.3. Esa figura muestra una vista de sección del sistema ideado para soportar el tubo de zafiro en el interior del resonador. Uno de los principales problemas es conseguir que el sello entre dos materiales diferentes sea el adecuado para evitar fugas a la hora de trabajar a altas presiones, para solventar este problema se ha empleado una pieza comercial de la marca HIP 21AF1AF2 que se mecanizó para adaptar el tubo, una serie de juntas de PFTE que junto una serie de juntas tóricas adaptadas, crean el sello y el ajuste adecuado para mantener la presión. Dos platos de acero 316 y 8 separadores del mismo material mantiene alineado el conjunto de sujeción (figura 4.3) del tubo con el eje central del resonador. 


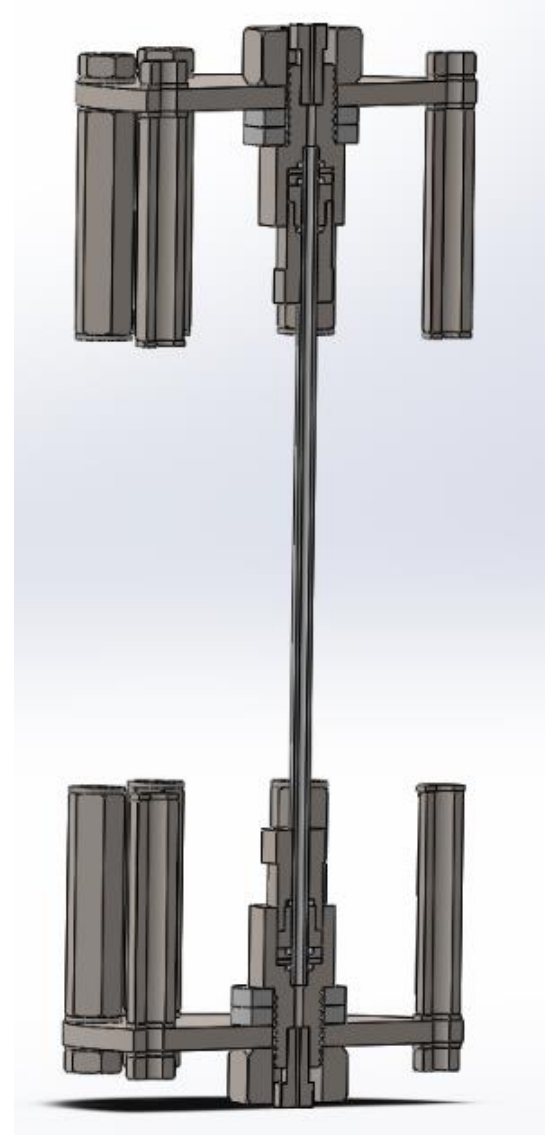

Figura 4.3 Vista de sección del sistema de sujeción del tubo de zafiro.

Los diagramas técnicos, así como fotografías de la cavidad cilíndrica y todos los accesorios que conforman el equipo se muestran en el anexo 1 de la presente memoria. Desde los extremos de este dispositivo se han acoplado las conexiones necesarias para introducir las muestras en el interior del tubo, para ello se han dispuesto de dos bombas de tipo jeringa modelo ISCO 260D controladas por el equipo Pump-Controller de la misma marca comercial, mediante un modo de funcionamiento a caudal o a presión constante con un rango de funcionamiento desde $1 \mu \mathrm{l} / \mathrm{min}$ a $90 \mu \mathrm{l} / \mathrm{min}$ y con una presión máxima de $51.7 \mathrm{MPa}$. Tanto el circuito que conduce la muestra hasta el resonador como las bombas de jeringa se 
encuentran termostatizadas a $298.15 \mathrm{~K}$ con el propósito de evitar un posible cambio de fase en el circuito, en lugar de en el interior del resonador, para ello se ha dispuesto un baño termostático externo de la marca comercial JULABO F25. En la figura 4.4; donde se presenta un diagrama esquemático de la instalación, se muestra de manera coloreada el circuito de termostático por agua empleado.

\subsection{El sistema experimental}

La celda de medida se encuentra sumergida en un baño termostático Fluke modelo 7037 que permite trabajar en un rango de temperatura de (233.15 a 423.15) $\mathrm{K}$ con una homogeneidad de $\pm 0.01{ }^{\circ} \mathrm{C}$. El fluido en el que se encuentra inmerso la celda de medida es etanol con el propósito de conseguir temperaturas inferiores a $0{ }^{\circ} \mathrm{C}$. La figura 4.4 muestra un diagrama del equipo experimental, y a lo largo de esta sección se describirán las partes del mismo.

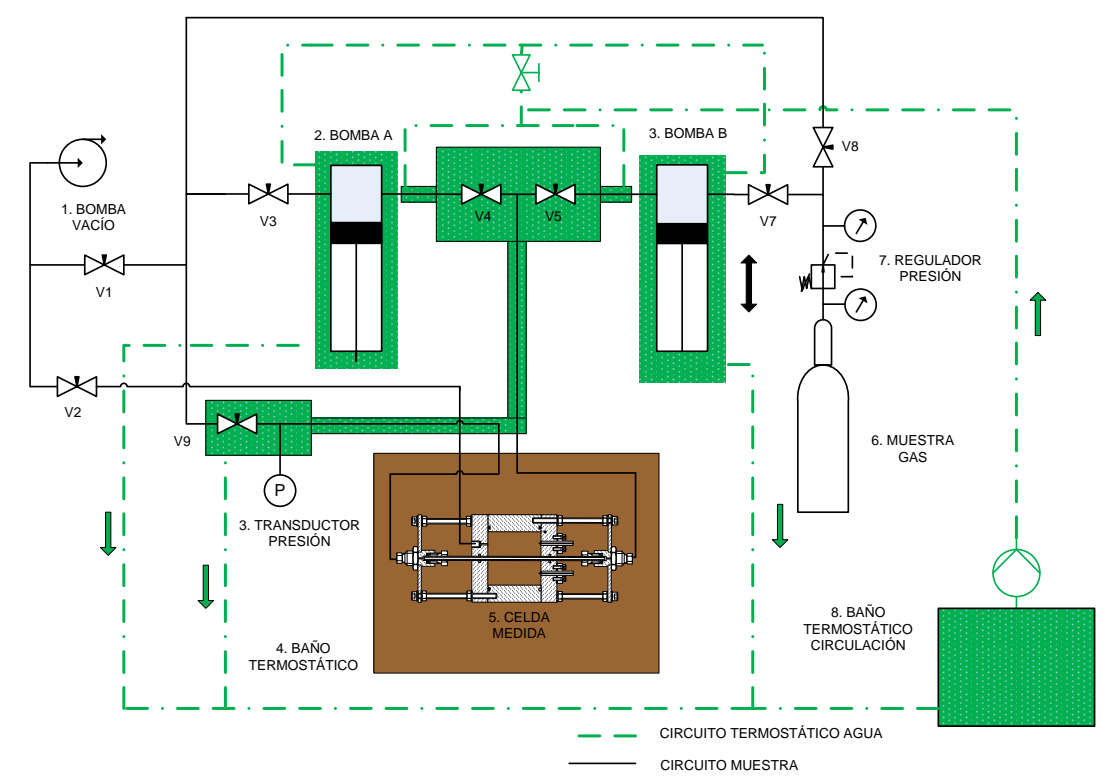

Figura 4.4 Diagrama esquemático del equipo experimental y equipos auxiliares empleados en esta Tesis Doctoral. 
En la figura 4.5 se muestran unas fotografías del equipo experimental empleado en esta tesis doctoral. Como puede observarse, este se encuentra ubicado en una estructura de aluminio dividida en dos niveles, en el nivel inferior se sitúan las muestras gaseosas para su estudio, la bomba de vacío y el baño para el circuito termostático; en el nivel superior, se encuentran los dispositivos de medida de temperatura, presión y el baño termostático en el que se encuentra sumergida la celda de medida.
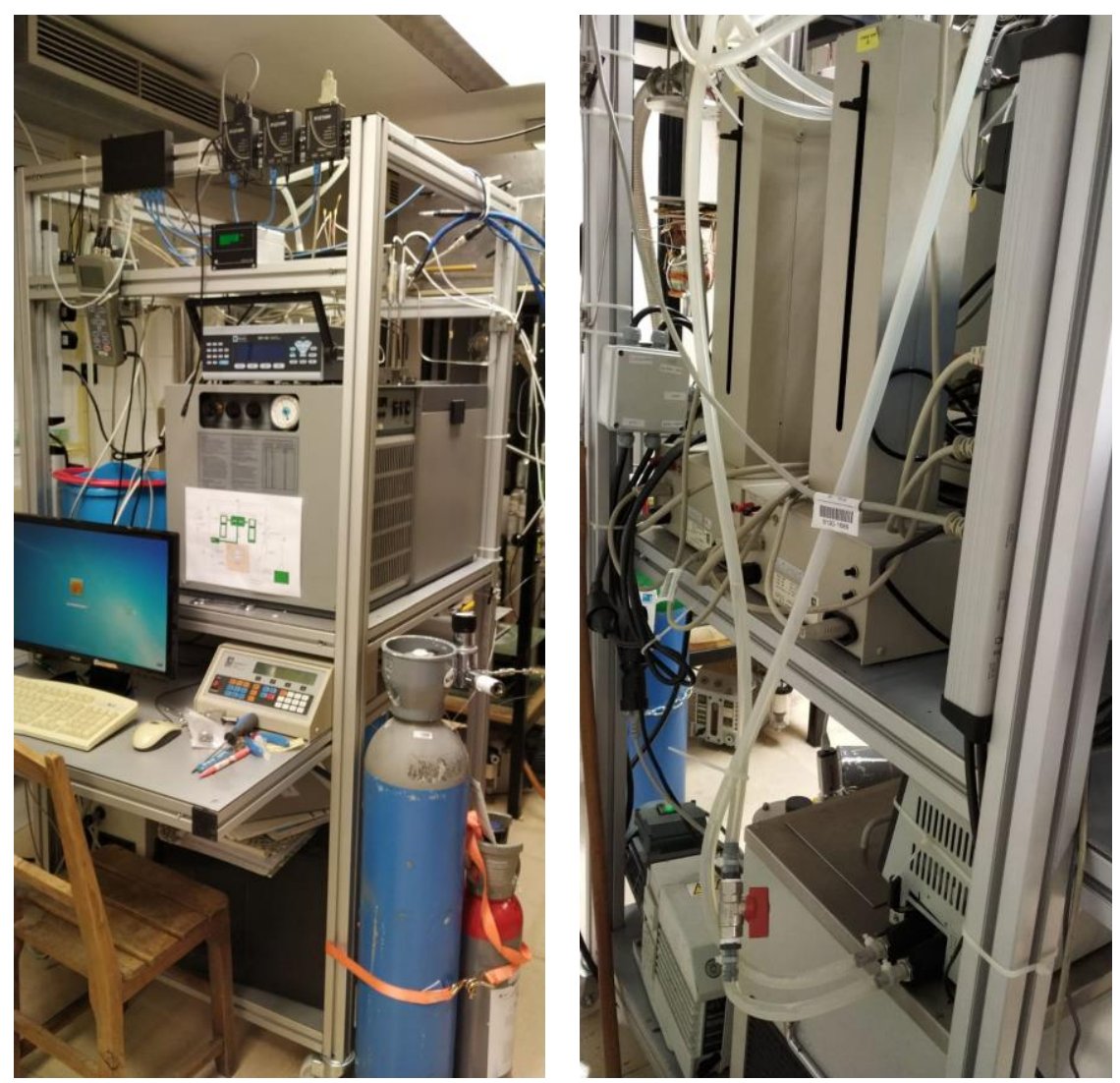

Figura 4.5 Vista frontal (izquierda) y trasera (derecha) del equipo experimental empleado en esta Tesis Doctoral. 


\subsubsection{El analizador vectorial de redes (VNA)}

Junto con la celda de medida, el VNA ha sido una de las piezas más importantes para la elaboración de esta tesis doctoral. El equipo empleado ha sido un Analizador Vectorial de Redes de dos puertos, uno para el estímulo y otro para la respuesta, modelo PNL-N5230C de Agilent Technologies, con un rango de frecuencia de $300 \mathrm{kHz}$ a $13.5 \mathrm{GHz}$ de dos puertos con una potencia de salida máxima de $+6 \mathrm{dBm}$ a $+11 \mathrm{dBm}$ y con medida de los parámetros de dispersión $\mathrm{S}$. La función principal de este instrumento es de analizar eléctricamente la respuesta de un dispositivo a una señal de estímulo sinusoidal abarcando el rango de radiofrecuencias y microondas, la cavidad cilíndrica ha sido el dispositivo a testear mediante el analizador de redes.

Para poder realizar la medida en los parámetros de dispersión S, es necesario establecer los planos de referencia de cada uno de los puertos, en esencia, es necesario eliminar en la medida de lo posible aquellos errores sistemáticos que puedan originarse en la medida. Para ello el conjunto de cables, conectores y el propio VNA necesitan ser calibrados. La calibración se ha realizado en el transcurso del montaje de todos los elementos del equipo experimental, recurriendo al kit de calibración mecánico suministrado por Agilent Technologies 85052D. El estándar de calibración en los dos puertos se ha llevado a cabo empleando cuatro dispositivos de prueba llamados OPEN (red abierta), SHORT (red en corto circuito), LOAD (red en carga) para calibrar la reflexión y THRU (red conectada) para calibrar la transmisión, los cuales deben ser conectados a los puertos del analizador para que este pueda comparar y establecer la diferencia entre diferentes modos.

Medir la frecuencia de resonancia y el factor de calidad de la cavidad en un determinado modo es el objetivo principal de esta tesis doctoral. El modo se elige de modo que la distribución del campo electromagnético en el interior del tubo que contiene la muestra provoque los mayores cambios en los parámetros de 
resonancia con el cambio de la fase del fluido que se encuentra en su interior. La discontinuidad en la pendiente de la frecuencia frente a la presión o la temperatura, es causada por el cambio en las propiedades dieléctricas del fluido en el tubo de muestra cuando sucede una transición de fase de primer orden, por lo tanto, para mediciones de punto de burbuja y rocío, se utilizó un procedimiento de seguimiento simple de la frecuencia de resonancia.

\subsubsection{Instrumentos para la medida de la temperatura}

La temperatura de la cavidad cilíndrica resonante ha sido medida mediante dos sondas de platino a $100 \Omega$ clase B de referencia 611.7867 de RS-Amidata montadas sobre varillas de acero inoxidable y ubicadas en el interior del baño termostático a ambos lados de la cavidad como se muestra en la figura 4.4, un puente ASL F100 es el encargado de realizar las lecturas de las temperaturas.

Las sondas de temperatura se calibraron "in situ" con un baño portátil y se calcularon sus constantes de acuerdo a la escala internacional de temperatura ITS90 [71]. La incertidumbre relativa expandida $(k=2)$ en la medición de temperatura se estimó en menos de $30 \mathrm{mK}$, para el rango de temperatura de $233.15 \mathrm{~K}$ a 383.15 K.

\subsubsection{Instrumentos para la medida de la presión}

La presión fue medida mediante un transductor externo de presión absoluta modelo PDCR 911-175 de tipo piezoresistivo, conectado a un indicador multifunción Druck DPI 145. El sensor cubre el rango de presión operacional del instrumento de hasta $70 \mathrm{MPa}$, y se ha calibrado en el laboratorio TERMOCAL con trazabilidad a partir de patrones primarios. La incertidumbre estándar de calibración en la medición de la presión de $\mathrm{u}(\mathrm{p})=200 \cdot 10^{-4} * \mathrm{p}+200 \mathrm{~Pa}$ 


\subsection{Software de control y adquisición de datos}

La adquisición de datos experimentales es una tarea compleja si no se dispone de los equipos necesarios para tal fin, es por ello que todos los equipos auxiliares que forman parte de esta técnica se han conectado a un ordenador que nos permitirá almacenar y gestionar todos los datos experimentales que sean necesarios para poder obtener la información deseada. Dado que no todos los instrumentos de medida disponen de las mismas conexiones al ordenador se ha optado por unificar todos ellos mediante puertos Ethernet RJ45. Los equipos con conexiones RS232 como el sensor de presión, las bombas ISCO y el baño termostático donde está sumergida la celda, se han conectado a puertos Ethernet mediante dispositivos externos de la marca comercial WIZnet ${ }^{\text {TM }}$ modelo WIZ100 ${ }^{\text {TM }}$, que permiten transformar la conexión de entrada del puerto RS232 a una conexión de salida con un puerto RJ45, estos junto con el software de control que proporciona la misma marca comercial se han configurado para que sean reconocidos como elementos de red y por lo tanto controlados por el ordenador. Con esto y a excepción de lector de temperatura que se conecta mediante USB al ordenador, el resto de los dispositivos se interconectan por medio de un Switch de 8 puertos de la marca comercial D-Link ${ }^{\mathrm{T}}$. Con todos los equipos auxiliares conectados en red, por medio del escritorio remoto se puede acceder al equipo experimental y controlarlo desde cualquier ordenador.

El software empleado para gestionar el control del equipo experimental y recuperar todos los datos que estos equipos auxiliares proporcionan ha sido el VEE Pro $9.3^{\circledR}$ suministrado por Keysight Technologies. Partiendo del programa base que gestiona el VNA desarrollado por Ángel Gómez [15] se han programado el resto de rutinas capaces de controlar los equipos auxiliares más importantes para la medida del equilibrio de fases. Las figuras 4.6 a 4.8 muestran los paneles de control de los programas creados para el control y la adquisición de datos. 


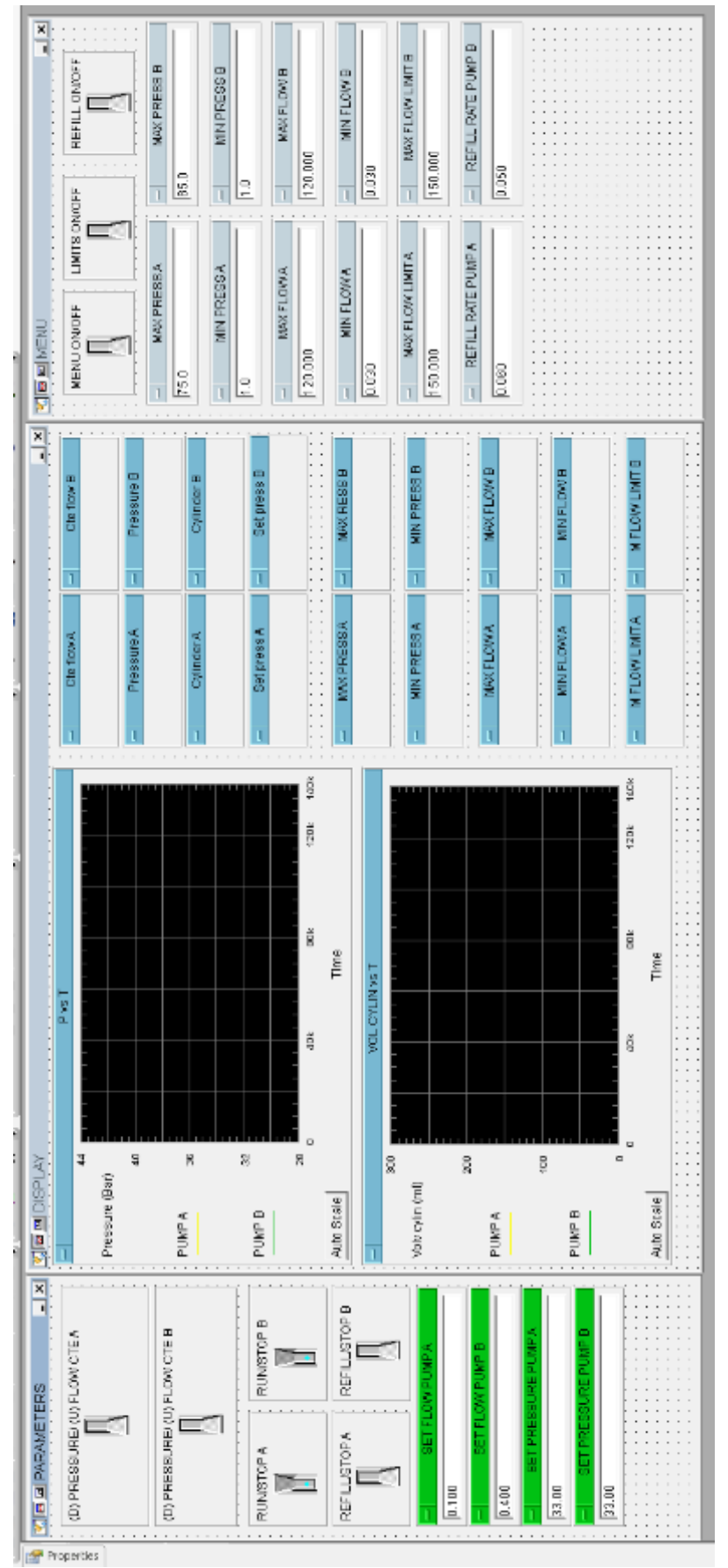

Figura 4.6

Panel de control automático para en funcionamiento de las bombas ISCO. 


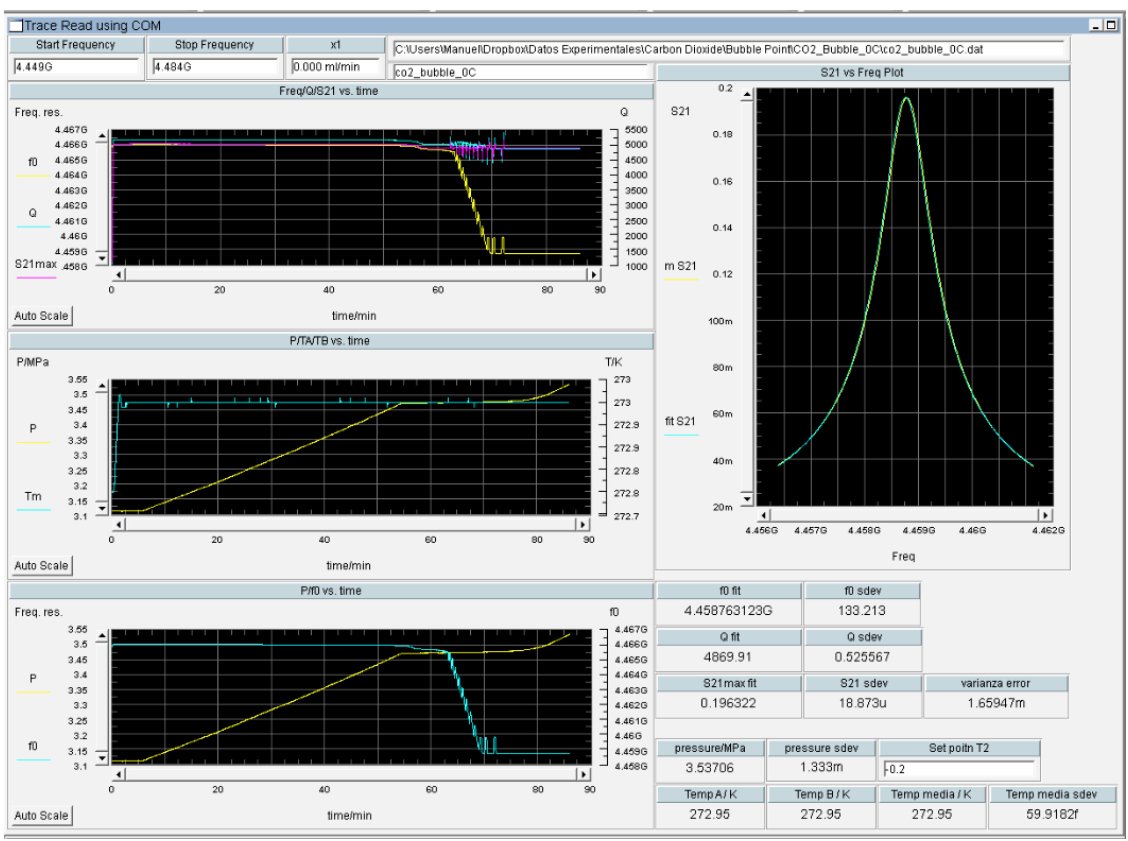

Figura 4.7 Panel para el control automático del VNA y adquisición de datos experimentales.

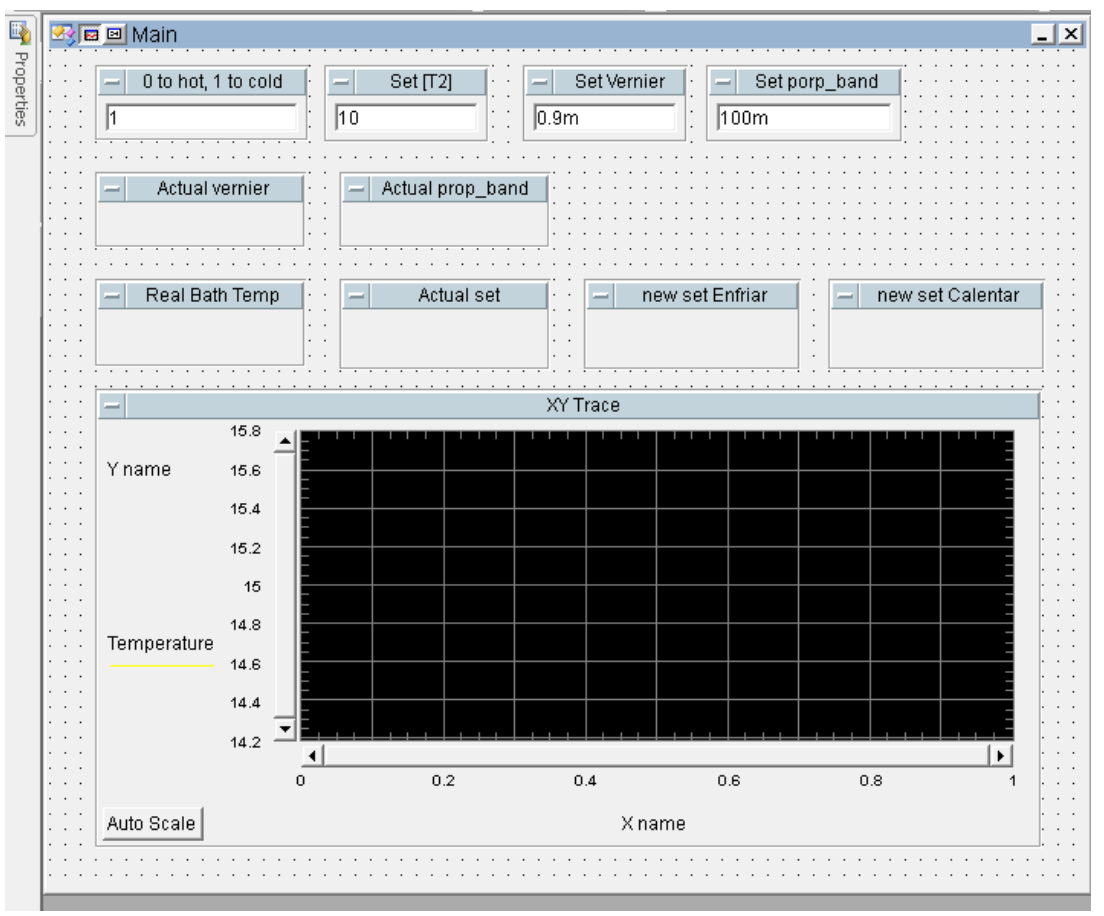

Figura 4.8 Panel para el control automático del baño termostático Fluke. 

CAPítulo 5

\section{Medidas con $\mathrm{CO}_{2}$}





\subsection{Introducción}

Cuando se desarrolla una nueva técnica experimental, ya sea por medio de una serie de modificaciones que en cierto modo mejoren dicha técnica, o por el diseño de una nueva técnica experimental, se hace necesario un procedimiento de validación que de veracidad a los datos experimentales que se pretenden obtener. Para lograr esto, se recurre a un fluido de referencia cuyas propiedades termodinámicas sean bien conocidas para que puedan ser comparadas con los resultados obtenidos con la nueva técnica. El dióxido de carbono ha sido la sustancia empleada para la validación de la técnica ya que es una sustancia de la que se conocen bien sus propiedades físicas y termodinámicas. Esta sustancia no presenta momento dipolar permanente, aunque si cuadrupolar y por tanto hay interacción electroestática entre sus moléculas, por lo que puede considerarse una sustancia no polar.

Esta sección se centrará en la determinación de la curva de vaporización (puntos de rocío y burbuja) comparando los datos experimentales con la ecuación de estado de Span \& Wagner [16] implementada en la base de datos en el NIST REFPROP [20]. Además los datos experimentales obtenidos se han correlacionados con las sus propiedades dieléctricas empleando para ello el procedimiento establecido por Harvey y Lemmon [21], las frecuencias de resonancia obtenidas en cada uno de los ensayos permitirán obtener el parámetro de proporcionalidad " $A$ ", empleando para ellos el método de las perturbaciones descrito en el capítulo 3.

\subsection{Procedimiento experimental}

El $\mathrm{CO}_{2}$ empleado es de la marca comercial Alphagaz suministrado por Air Liquid con una pureza del $99.999 \%$ con un contenido en impurezas inferior a $\leq 200 p p m$ en volumen. El procedimiento de medida consiste en ir modificando la presión a lo 
largo de una isoterma; la variación isotérmica se ha llevado a cabo empleando el baño termostático de la marca comercial Fluke modelo 7037 descrito en el apartado 4.3, el tiempo estimado para garantizar que el resonador se encuentra a la temperatura deseada ha sido de 2 horas a contar en el momento que el baño haya alcanzado la temperatura de trabajo. Una vez conectada la botella a la línea principal, se realiza el vacío en todo el circuito, incluida la cavidad y el tubo de zafiro que contendrá la muestra, este proceso continuará durante 1 hora a fin de asegurarse que se arrastran posibles impurezas tanto en la línea como en la cavidad.

Transcurrido este periodo de tiempo, se llenan con el $\mathrm{CO}_{2}$ las bombas de jeringa ISCO 260D, que serán las encargadas de realizar los desplazamientos positivos (determinación del punto de rocío) y negativos (determinación del punto de burbuja). La presión de partida, independientemente del cambio de fase que se desee determinar será, de un $20 \%$ menor o mayor del valor estimado para garantizar que la fase a la que se encuentre la muestra es gaseosa, si se quiere determinar el punto de rocío, o líquida si lo que se desea determinar es el punto de burbuja. El caudal que suministrarán las bombas será el suficiente para poder observar un cambio cuantificable en el momento del cambio de fase, este valor se ha establecido en $1.00 \mathrm{ml} / \mathrm{min}$ para ambos experimentos.

Partiendo siempre de la fase gaseosa, y en las condiciones descritas anteriormente, se comienza el experimento con desplazamiento positivo de las bombas (aumento de la presión) hasta alcanzar el cambio de fase (rocío), trascurrida 1 hora de espera para asegurar la homogeneidad en la nueva fase obtenida, se realiza el desplazamiento negativo (descenso de la presión) hasta volver a determinar el cambio de fase (burbuja). Una vez que se haya obtenido el dato experimental deseado, se devolverán las bombas a un estado que permita determinar un nuevo punto acondicionando el baño a las nuevas condiciones de temperatura. 


\subsection{Determinación de las incertidumbres}

La incertidumbre expandida combinada de las medidas de los puntos de burbuja y rocío en las condiciones de presión y temperatura se ha determinado acorde a la "Guía para la expresión de la incertidumbre de medida" (JCGM 106 2012) [19]. La medida de la incertidumbre expandida para la temperatura $U(T)$ en la medida de los puntos de rocío y burbuja puede ser estimada por:

$$
\mathrm{U}(\mathrm{T})=2 \cdot\left[\mathrm{u}\left(\mathrm{T}_{\text {res }}\right)^{2}+\mathrm{u}\left(\mathrm{T}_{\text {rep }}\right)^{2}+\mathrm{u}\left(\mathrm{T}_{\text {cal }}\right)^{2}\right]^{1 / 2}
$$

Donde $\mathrm{u}\left(\mathrm{T}_{\mathrm{res}}\right)$ representa la incertidumbre estándar debida a la resolución de medidor de temperatura, como ya se indicó en el apartado 4.3.2, un dispositivo ASL F100 es el encargado de obtener las lecturas de las sondas de temperaturas, este dispositivo tiene una resolución de $\mathrm{u}\left(\mathrm{T}_{\text {res }}\right)=0.001 \mathrm{~K}$. En cuanto a $\mathrm{u}\left(\mathrm{T}_{\text {rep }}\right)$ hace referencia a la incertidumbre estándar debida a la reproducibilidad de la medida de la temperatura en las condiciones en las que se origina el cambio de fase (rocío o burbuja). Por último, el término $\mathrm{u}\left(\mathrm{T}_{\text {cal }}\right)$, se establece como la incertidumbre estándar debida a la calibración de las sondas de temperatura empleadas en la medida en el apartado 4.3.2 se establece dicha incertidumbre en $\mathrm{u}\left(\mathrm{T}_{\text {cal }}\right)=0.03 \mathrm{~K}$.

Cuando se trata de determinar la incertidumbre expandida $U\left(\mathrm{~T}_{\mathrm{C}}\right)$ en el punto crítico es necesario introducir una nueva contribución a la ecuación 5.1, u( $\left.\mathrm{T}_{\text {interp }}\right)$, debida al polinomio de ajuste que se ha empleado con el fin de determinar, mediante interpolación de los datos experimentales, el punto crítico, quedando la ecuación anterior de la siguiente manera. 


$$
\mathrm{U}\left(\mathrm{T}_{\mathrm{C}}\right)=2 \cdot\left[\mathrm{u}\left(\mathrm{T}_{\text {res }}\right)^{2}+\mathrm{u}\left(\mathrm{T}_{\text {rep }}\right)^{2}+\mathrm{u}\left(\mathrm{T}_{\text {cal }}\right)^{2}+\mathrm{u}\left(\mathrm{T}_{\text {interp }}\right)^{2}\right]^{1 / 2}
$$

La incertidumbre expandida en la medida de la presión $U(p)$ de manera análoga a la incertidumbre expandida debida a la temperatura puede ser determinada a partir de la expresión:

$$
\mathrm{U}(\mathrm{p})=2 \cdot\left[\mathrm{u}\left(\mathrm{p}_{\text {res }}\right)^{2}+\mathrm{u}\left(\mathrm{p}_{\text {rep }}\right)^{2}+\mathrm{u}\left(\mathrm{p}_{\text {cal }}\right)^{2}+\mathrm{u}\left(\mathrm{p}_{\text {lineal }}\right)^{2}\right]^{1 / 2}
$$

De manera que, de forma análoga al cálculo de la incertidumbre estándar de la temperatura la incertidumbre debida a la resolución se establece en $\mathrm{u}\left(\mathrm{p}_{\mathrm{res}}\right)=$ 0.0001 MPa que es la resolución del indicador multifunción Druck DPI 145 (visto en el apartado 4.3.3). La incertidumbre estándar debida a la repetibilidad u( $\left.\mathrm{p}_{\mathrm{rep}}\right)$. Por último, la incertidumbre obtenida por la calibración del medidor, tal y como se comentó en el apartado 4.3.3 que fue $\mathrm{u}(\mathrm{p})=200 \cdot 10^{-4} * \mathrm{p}+200 \mathrm{~Pa}$. Debido al método estadístico empleado para la determinación de los puntos en los cambios de fase se hace necesario de introducir una contribución $u\left(\mathrm{p}_{\text {lineal }}\right)$ a la incertidumbre debido a la intersección de las rectas, siendo esta la mayor contribución.

De manera análoga a la determinación de la incertidumbre expandida de la temperatura crítica, el mismo polinomio de interpolación se emplea para la determinación de la presión crítica, por lo que es necesario introducir en sustitución de la contribución a debida a la intersección de las rectas $\mathrm{u}\left(\mathrm{p}_{\text {lineal }}\right)$, una contribución $\mathrm{u}\left(\mathrm{p}_{\text {interp }}\right)$ debido al polinomio de ajuste utilizado en la determinación, por interpolación de los datos experimentales de la presión crítica quedando la ecuación 5.3 de la siguiente manera: 


$$
\mathrm{U}\left(\mathrm{p}_{\mathrm{C}}\right)=2 \cdot\left[\mathrm{u}\left(\mathrm{p}_{\text {res }}\right)^{2}+\mathrm{u}\left(\mathrm{p}_{\text {rep }}\right)^{2}+\mathrm{u}\left(\mathrm{p}_{\text {cal }}\right)^{2}+\mathrm{u}\left(\mathrm{p}_{\text {interp }}\right)^{2}\right]^{1 / 2}
$$

La tabla 5.1 muestra el cálculo obtenido para la determinación de la incertidumbre expandida $(k=2)$ en la determinación de la curva de vaporización del dióxido de carbono, a diferencia de los cálculos para la determinación en la incertidumbre en el punto crítico, y tal como se comentó al principio de este apartado, en sustitución de la contribución a debida a la intersección de las rectas $\mathrm{u}\left(\mathrm{p}_{\text {lineal }}\right)$, una contribución $\mathrm{u}\left(\mathrm{p}_{\text {interp }}\right)$ debido al polinomio de ajuste utilizado en la determinación del punto crítico

Tabla 5.1 Tabla de incertidumbre de medidas para la determinación de la curva de vaporización del $\mathrm{CO}_{2}$ en el intervalo de temperaturas de (273.35-302.35) K y presiones (3.502-7.077) MPa. Factor de cobertura k=2.

\begin{tabular}{|c|c|c|c|c|c|}
\hline & Fuente & Unidad & Estimado & Divisor & $\mathrm{u}(\mathrm{x}) / \mathrm{MPa}$ \\
\hline \multirow{4}{*}{$u(p)$} & Resolución & \multirow{4}{*}{$\mathrm{MPa}$} & 0.0001 & 1 & 0.0001 \\
\hline & Repetibilidad & & 0.001 & 1 & 0.001 \\
\hline & Calibración & & 0.0002 & 1 & 0.0002 \\
\hline & Lineal & & 0.003 & 1 & 0.003 \\
\hline \multirow{3}{*}{$u(T)$} & Resolución & \multirow{3}{*}{$\mathrm{MPa}$} & 0.01 & 1 & 0.01 \\
\hline & Repetibilidad & & 0.001 & 1 & 0.0001 \\
\hline & Calibración & & 0.03 & 1 & 0.03 \\
\hline$U(p)$ & & $\mathrm{MPa}$ & & $k=2$ & 0.010 \\
\hline$U(T)$ & & $\mathrm{K}$ & & $k=2$ & 0.05 \\
\hline
\end{tabular}


La tabla 5.2 muestra el cálculo realizado para la determinación de la incertidumbre expandida $(\mathrm{k}=2)$ en la determinación del punto crítico y toma las contribuciones de presión y temperatura, al tratarse de una sustancia pura que proviene de una muestra comercial, se conoce la cantidad de impurezas que contiene, pero no su composición molar. La mayor contribución a esta incertidumbre se debe, en primer término, a la calibración de los instrumentos de medida, y en un segundo término al polinomio de ajuste empleado para la determinación del punto crítico.

Tabla 5.2 Tabla de incertidumbre para las medidas del punto crítico en el intervalo de temperaturas de (303.36304.04) K y presiones (7.238-7.360) MPa. Factor cobertura k=2.

\begin{tabular}{|c|c|c|c|c|c|}
\hline & Fuente & Unidad & Estimado & Divisor & $\mathrm{u}(\mathrm{x}) / \mathrm{MPa}$ \\
\hline \multirow{4}{*}{$u\left(p_{c}\right)$} & Resolución & \multirow{4}{*}{$\mathrm{MPa}$} & 0.0001 & 1 & 0.0001 \\
\hline & Repetibilidad & & 0.003 & 1 & 0.004 \\
\hline & Calibración & & 0.002 & 1 & 0.0017 \\
\hline & interpolación & & 0.003 & 1 & 0.003 \\
\hline \multirow{4}{*}{$u\left(T_{c}\right)$} & Resolución & \multirow{4}{*}{$\mathrm{MPa}$} & 0.001 & 1 & 0.001 \\
\hline & Repetibilidad & & 0.001 & 1 & 0.001 \\
\hline & Calibración & & 0.03 & 1 & 0.025 \\
\hline & interpolación & & 0.003 & 1 & 0.003 \\
\hline$U\left(p_{c}\right)$ & \multicolumn{3}{|c|}{$\mathrm{MPa}$} & $k=2$ & 0.010 \\
\hline$U\left(T_{c}\right)$ & \multicolumn{3}{|c|}{$\mathrm{K}$} & $k=2$ & 0.05 \\
\hline
\end{tabular}

\subsection{Resultados experimentales}

Las figuras 5.1 y 5.2, muestran las gráficas de los resultados obtenidos al medir el cambio de fase del dióxido de carbono en condiciones isotérmicas a $298.35 \mathrm{~K}$, de la presión y de la frecuencia normalizada (ecuación 3.36) frente a la frecuencia de resonancia para los puntos de burbuja y rocío respectivamente. El cambio de fase se caracteriza, en primer lugar, por un cambio en la frecuencia de resonancia en el interior de la cavidad originado por un cambio en la constante dieléctrica de 
la sustancia, así mismo durante el cambio de fase la presión de la sustancia permanece constante.

Tal y como se estableció en el apartado 3.2 de la presente tesis doctoral, la técnica presentada pertenece al método sintético con detección de cambio de fase y no visual, por lo que es necesario elaborar un procedimiento que permita establecer el valor de punto de burbuja o rocío de manera sistemática, es por ello que, dado que en el cambio de fase se origina una discontinuidad, podemos determinar el valor de los puntos de rocío y burbuja de los datos experimentales a partir de los valores de la meseta. Para ello se establece la condición, por la cual se considera que la zona de líquido o vapor saturado antes del cambio de fase puede ser ajustada a un polinomio de segundo grado, y dado que en el cambio de fase la presión permanece constante, la intersección entre ambas rectas dará como resultado el punto de burbuja o rocío deseado. Durante la medida experimental no se han encontrado fases metaestables coincidiendo de manera bastante precisa con mesetas en los cambios de fase muy bien definidas.

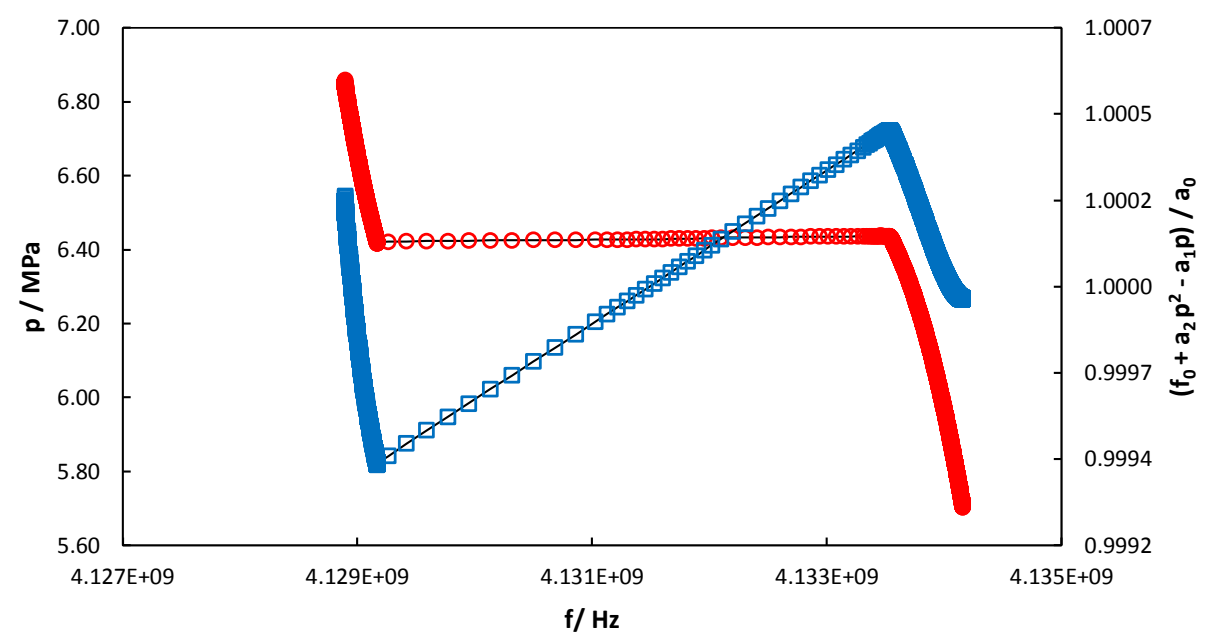

Figura 5.1 Presión (o) y frecuencia normalizada ( $\square$ ) frente a la frecuencia de resonancia en la determinación del punto de burbuja a $298.35 \mathrm{~K}$. 


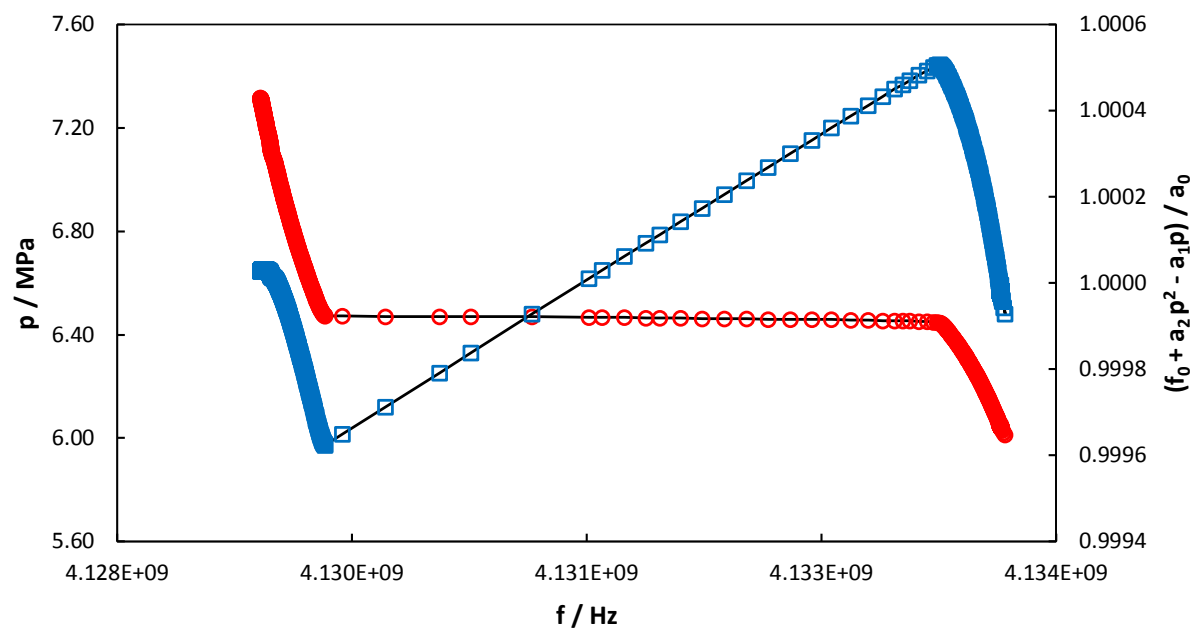

Figura 5.2 Presión (o) y frecuencia normalizada ( $\square$ ) frente a la frecuencia de resonancia en la determinación del punto de rocío a $298.35 \mathrm{~K}$.

Las figuras 5.1 y 5.2 muestran claramente que la condición anteriormente comentada puede ser tomada en consideración, además se ve claramente un tramo de presión constante que corresponde al cambio de fase, por lo que la técnica que se presenta determina de manera acertada en ambos casos el cambio de fase.

La tabla 5.3 muestra los resultados experimentales de los puntos de burbuja, rocío y frecuencias de resonancia del dióxido de carbono y su comparación con los valores calculados mediante la ecuación de estado Span \& Wagner [16]. 
Tabla 5.3 Datos experimentales de los puntos de burbuja, rocío y frecuencia de resonancia del dióxido de carbono con una incertidumbre expandida $(k=2)$ en $U(T)=0.05 K ; U(p)=0.006 \mathrm{MPa}$. Presiones calculadas mediante la ecuación de estado de Span \& Wagner [16].

\begin{tabular}{l|rcccc} 
Ensayo & $\mathbf{T}_{\text {exp }} / \mathbf{K}$ & $\mathbf{p}_{\text {exp }} / \mathbf{M P a}$ & $\mathbf{f}_{\text {exp }} / \mathbf{G H z}$ & $\mathbf{p}_{\text {Eos }} / \mathbf{M P a}$ & $\delta \mathbf{p} \%$ \\
\hline Burbuja & 273.35 & 3.502 & 4.1302 & 3.503 & -0.048 \\
Burbuja & 278.36 & 3.989 & 4.1299 & 3.991 & -0.041 \\
Burbuja & 283.35 & 4.521 & 4.1297 & 4.525 & -0.090 \\
Burbuja & 288.35 & 5.107 & 4.1295 & 5.112 & -0.090 \\
Burbuja & 293.36 & 5.753 & 4.1293 & 5.757 & -0.084 \\
Burbuja & 298.35 & 6.461 & 4.1293 & 6.464 & -0.046 \\
Burbuja & 300.35 & 6.762 & 4.1294 & 6.767 & -0.070 \\
Burbuja & 302.35 & 7.076 & 4.1296 & 7.083 & -0.099 \\
Rocío & 273.35 & 3.504 & 4.1330 & 3.503 & 0.015 \\
Rocío & 278.36 & 3.992 & 4.1328 & 3.991 & 0.037 \\
Rocío & 283.35 & 4.526 & 4.1324 & 4.525 & 0.030 \\
Rocío & 288.35 & 5.115 & 4.1322 & 5.112 & 0.055 \\
Rocío & 293.36 & 5.760 & 4.1320 & 5.757 & 0.039 \\
Rocío & 298.35 & 6.462 & 4.1316 & 6.464 & -0.031 \\
Rocío & 300.35 & 6.766 & 4.1313 & 6.767 & -0.017 \\
Rocío & 302.35 & 7.077 & 4.1310 & 7.083 & -0.081 \\
Sp \% $=\left(\frac{p_{\text {exp }}}{\mathrm{p}_{\text {Eos }}}-1\right) * 100$ & & & &
\end{tabular}

la incertidumbre expandida combinada para un factor de cobertura de $k=2$ en la medida de la temperatura fue de $\mathrm{U}(\mathrm{T})=0.05 \mathrm{~K}$ y en la medida de la presión de $U(p)=0.010 \mathrm{MPa}$ respecto de las ecuación de estado Span \& Wagner [16], mientras que la incertidumbre relativa en el rango de (3.502 a 7.077) $\mathrm{MPa}$ fue de $\mathrm{U}_{\mathrm{r}}(\mathrm{p})=$ $0.22 \%(\mathrm{MPa} / \mathrm{MPa})$ y $\mathrm{U}_{\mathrm{r}}(\mathrm{p})=0.11 \%(\mathrm{MPa} / \mathrm{MPa})$ respectivamente. Los valores de la temperatura se han obtenido una vez que tanto el baño como la cavidad se encontraban en las condiciones de temperatura deseada para llevar a cabo el ensayo, para ello una vez alcanzado el valor esperado, se han tomado 100 valores a intervalos de 5 segundos y se ha realizado la media aritmética, este proceso se ha repetido en tres ocasiones, antes de comenzar las rampas de presión, durante la medida experimental cerca del valor donde el cambio de fase debería ocurrir, y 
después de finalizar la medida, se han comparado los tres resultados observando que no ha habido cambios que dieran motivo para suponer que las condiciones de temperatura no fueran las esperadas.

La figura 5.3 muestra los datos experimentales comparados con la literatura Kim et al. [72], Saleh et al. [73], y Gómez [15] en el rango de temperaturas de $273.15 \mathrm{~K}$ hasta $304.00 \mathrm{~K}$ cercano a las inmediaciones del punto crítico, y también se comparan con la ecuación de estado del $\mathrm{CO}_{2}$ desarrollada por Span \& Wagner [16] implementada en el software REFPROP [20].

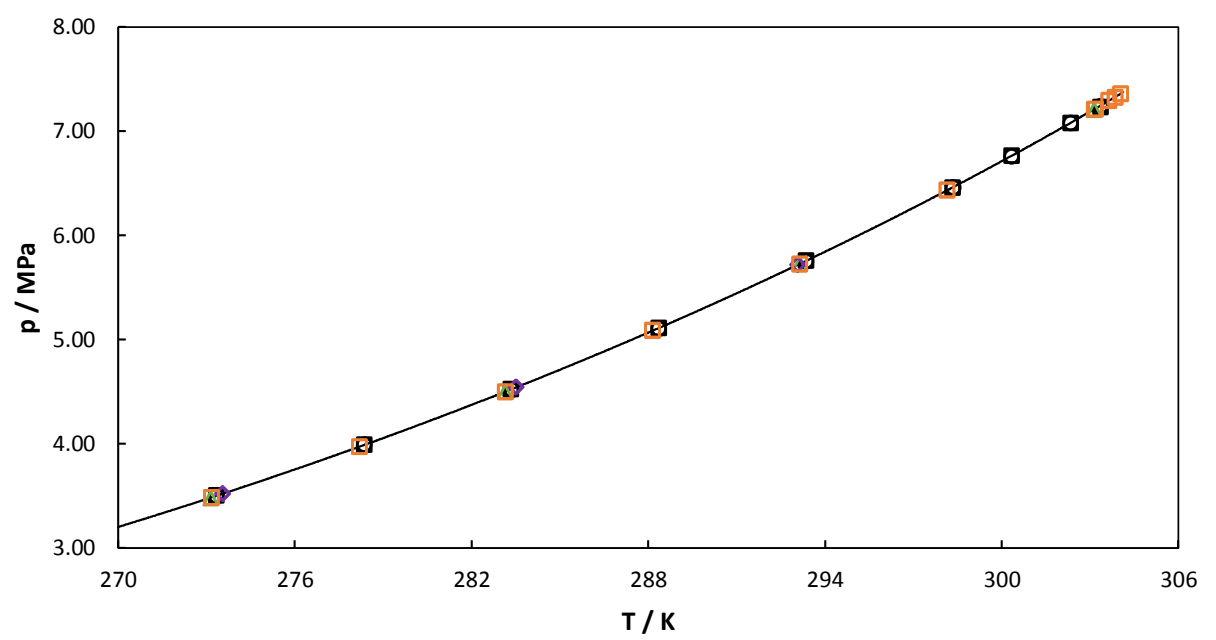

Figura 5.3Resultados experimentales en el rango de temperaturas de $273.35 \mathrm{~K}$ a $304.04 \mathrm{~K}$ de los puntos de burbuja (o), y rocío ( $\square$ ) para el $\mathrm{CO}_{2}$, y su comparación con la literatura Kim et al. [72] $(\Delta)$; Saleh et al. [73] ( $(\bullet)$; Gómez, [15] ( $\square$ ); datos de la referencia de Span \& Wagner (-) [16].

La figura 5.4 muestra gráficamente las desviaciones porcentuales de la ecuación de estado Span \& Wagner [16] con los datos experimentales de esta tesis doctoral y de la literatura Kim et al. [72], Saleh et al. [73], y Gómez [15] frente a la presión. Realizando un análisis de los resultados obtenidos se puede apreciar que los valores de Kim et al. [72] tienen desviaciones porcentuales promedio de $0.17 \%$ empleando para la determinación de estos valores una celda de equilibrio, 
mientras que para los valores medidos por Saleh et al. [73] las desviaciones porcentuales promedio son del $0.02 \%$ empleando para la determinación de las densidades un densímetro de hundimiento simple. Los valores obtenidos por Gómez [15]. empleando un equipo similar al propuesto en esta tesis doctoral proporciona unas desviaciones promedio de $0.03 \%$. En esta tesis doctoral se han obtenido unas desviaciones porcentuales promedio para los datos experimentales en la determinación del punto de burbuja de $0.07 \%$, mientras que en la determinación de punto de rocío las desviaciones porcentuales obtenidas fueron del $0.04 \%$.

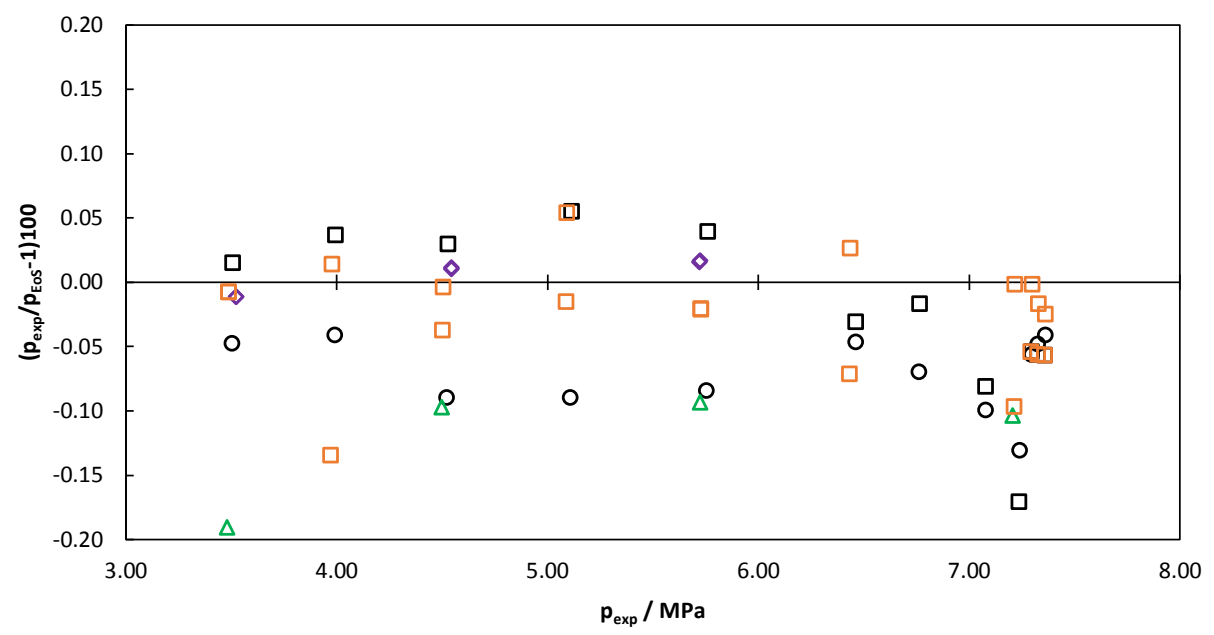

Figura 5.4Desviaciones porcentuales para los datos experimentales del $\mathrm{CO}_{2}$ de los puntos de burbuja (o), y rocío

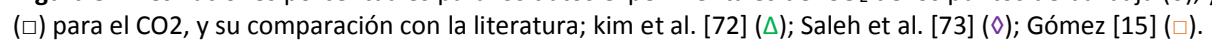

A partir de los datos experimentales de frecuencia obtenidos, podemos realizar una aproximación en la determinación del punto crítico del $\mathrm{CO}_{2}$. En la tabla 5.4 se muestran los resultados experimentales de presión temperatura y frecuencia de resonancia en las inmediaciones del punto crítico, y su comparación con los valores calculados mediante la ecuación de estado Span \& Wagner [16]. 
Tabla 5.4 Datos experimentales de los puntos de burbuja, rocío y frecuencia de resonancia del dióxido de carbono en las inmediaciones del punto crítico con una incertidumbre expandida $(k=2)$ en $U(T)=0.05 K ; U(p)=0.010 \mathrm{MPa}$. Presiones calculadas mediante la ecuación de estado de Span \& Wagner [16].

\begin{tabular}{l|ccccc} 
& $\mathbf{T}_{\text {exp }} / \mathbf{K}$ & $\mathbf{p}_{\text {exp }} / \mathbf{M P a}$ & $\mathbf{f}_{\text {exp }} / \mathbf{G H z}$ & $\mathbf{p}_{\text {Eos }} / \mathbf{M p a}$ & $\delta \mathbf{p} \%$ \\
\hline Burbuja & 303.36 & 7.238 & 4.1297 & 7.248 & -0.131 \\
Burbuja & 303.66 & 7.293 & 4.1298 & 7.298 & -0.056 \\
Burbuja & 303.83 & 7.326 & 4.1299 & 7.327 & -0.020 \\
Burbuja & 304.04 & 7.360 & 4.1300 & 7.363 & -0.046 \\
Rocío & 303.36 & 7.235 & 4.1308 & 7.248 & -0.171 \\
Rocío & 303.66 & 7.295 & 4.1307 & 7.298 & -0.052 \\
Rocío & 303.83 & 7.326 & 4.1306 & 7.327 & -0.018 \\
Rocío & 304.04 & 7.358 & 4.1305 & 7.363 & -0.062 \\
$\delta p \%=\left(\frac{p_{\text {Exp }}}{\mathbf{p}_{\text {EoS }}}-1\right) * 100$ & & & &
\end{tabular}

la desviación respecto de las ecuación de estado Span \& Wagner [16], resulta inferior a incertidumbre $\mathrm{U}_{\mathrm{r}}(\mathrm{p})=0.29 \%(\mathrm{MPa} / \mathrm{MPa})$. En la figura 5.5 se muestran los valores en el rango de temperatura cercanas al punto crítico, comparados con la ecuación de estado del $\mathrm{CO}_{2}$ desarrollada por Span \& Wagner [16] e implementada en el software REFPROP [20] y valores literatura proporcionada por Kim et al. [72], y Gómez, [15]. Además, se representa el valor de referencia del punto crítico que la ecuación de estado anteriormente descrita devuelve.

Para poder determinar el valor extrapolado del punto crítico en esta tesis doctoral, se han ajustado los datos experimentales de presión y temperatura con respecto a la frecuencia de resonancia a un polinomio de segundo grado como el que se muestra en la figura 5.6, el máximo de dicho polinomio nos devolverá el valor de la frecuencia de resonancia del punto crítico, y por lo tanto, el valor de la temperatura o presión crítica cuando ajustamos dicho polinomio a los valores de frecuencia de resonancia frente a la presión o temperatura. 


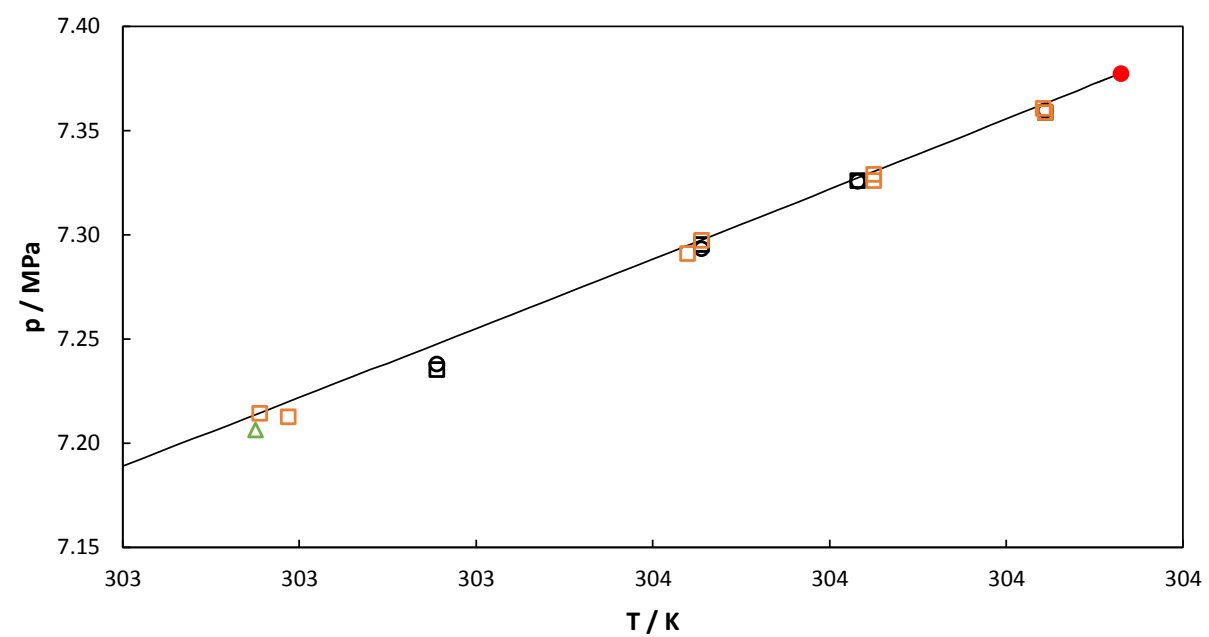

Figura 5.5 Resultados experimentales en las inmediaciones del punto crítico del $\mathrm{CO}_{2}$ de los puntos de burbuja (o), y rocío ( $\square$ ) para el $\mathrm{CO}_{2}$, y su comparación con la literatura; kim et al. [72] ( $\Delta$ ); Gómez, [15] ( $\square$ ); datos desde la referencia de Span \& Wagner $(-)$ y punto crítico $(\bullet)[16]$.

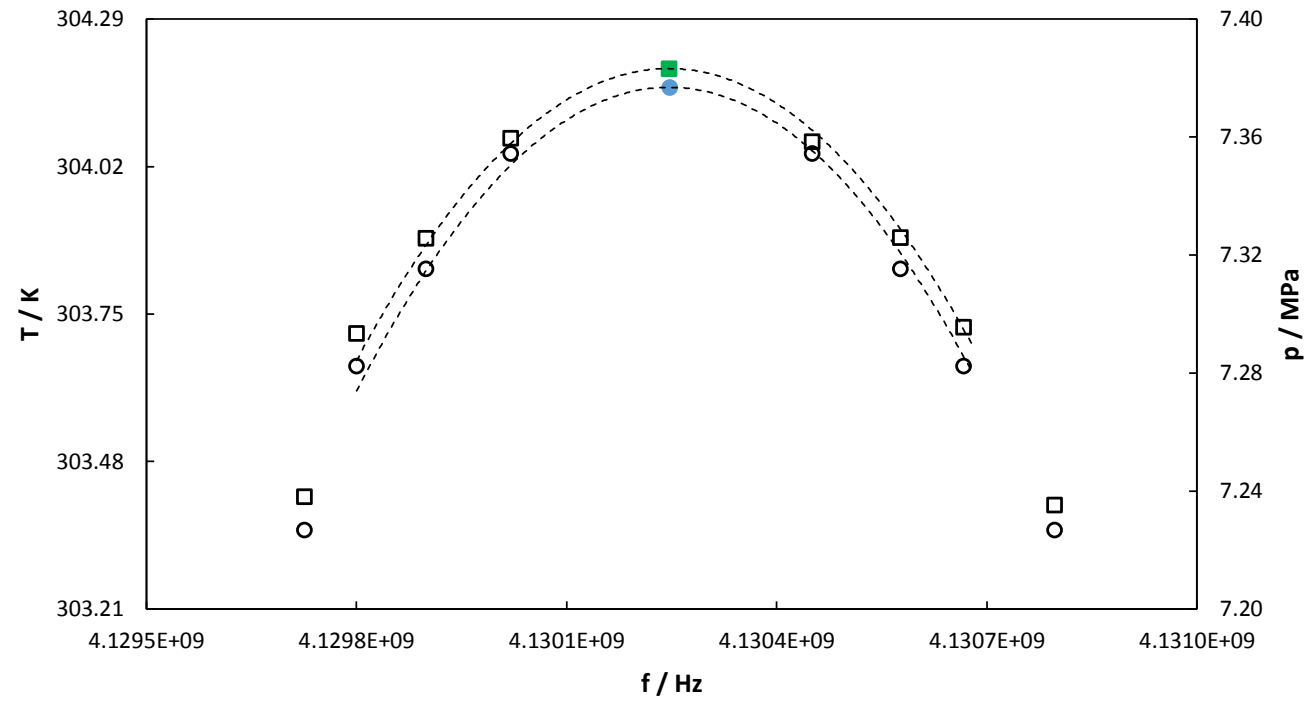

Figura 5.6Datos experimentales en las inmediaciones del punto crítico, de la temperatura de burbuja (izquierda) y rocío (derecha) (o) y a la presión de burbuja y rocío ( $\square$ ) frente a la frecuencia de resonancia. Polinomio de ajuste

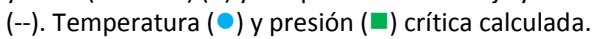


La tabla 5.5 se muestran los valores obtenidos del punto crítico del $\mathrm{CO}_{2}$ calculado tal y como se especificó en el apartado 5.3 de la presente memoria. Se han comparado estos datos con los proporcionados por la literatura existente

Tabla 5.5 Valores calculados del punto crítico, y su correspondiente incertidumbre expandida ( $k=2$ ), comparada con la literatura.

\begin{tabular}{l|cccc} 
& $\mathbf{T}_{\mathrm{c}} / \mathbf{K}$ & $\mathbf{U}\left(\mathbf{T}_{\mathrm{c}}\right) / \mathbf{K}$ & $\mathbf{p}_{\mathrm{c}} / \mathbf{M P a}$ & $\mathbf{U}\left(\mathbf{p}_{\mathrm{c}}\right) / \mathbf{M P a}$ \\
\hline Esta tesis doctoral & 304.17 & 0.06 & 7.383 & 0.01 \\
Gómez. [15] & 304.22 & 0.28 & 7.388 & 0.049 \\
Gil et al. [74] & 304.21 & 0.08 & 7.383 & 0.007 \\
Suehiro et al. [75] & 304.18 & 0.02 & 7.380 & 0.01 \\
Diefenbacher et al. [76] & 304.16 & 0.06 & 7.379 & 0.006 \\
Span\&Wagner et al. [16] & 304.13 & 0.01 & 7.373 & 0.0003
\end{tabular}

Como puede observarse, los valores obtenidos mediante el procedimiento empleado devuelven valores no muy alejados de los encontrados en la literatura e inferiores a la incertidumbre de medida. En esta tesis doctoral se ha obtenido, el valor correspondiente a la frecuencia de resonancia cuyo valor ha sido de 4.1302 $\mathrm{GHz}$, para el máximo del polinomio de ajuste, tal y como se indicó anteriormente. La tabla 5.6 muestra los coeficientes de ajuste obtenidos en la determinación del punto crítico.

Tabla 5.6 Coeficientes de ajuste y su desviación estándar $(\sigma)$ del polinomio de ajuste en la determinación de la $T_{c}$ y $P_{c}$ con respecto a la frecuencia.

\begin{tabular}{l|cccc} 
& $\mathbf{A}_{\mathbf{0}}$ & $\mathbf{A}_{\mathbf{1}}$ & $\mathbf{A}_{\mathbf{2}}$ & $\boldsymbol{\sigma}$ \\
\hline $\mathbf{T}_{\mathbf{c}}$ vs frecuencia $(\mathrm{Hz} / \mathrm{K})$ & $-2.803 \cdot 10^{-12}$ & $2.316 \cdot 10^{-2}$ & $-4.782 \cdot 10^{7}$ & 0.031 \\
$\mathbf{p}_{\mathbf{c}}$ vs frecuencia $(\mathrm{Hz} / \mathrm{MPa})$ & $-5.011 \cdot 10^{-13}$ & $4.139 \cdot 10^{-3}$ & $-8.548 \cdot 10^{6}$ & 0.005 \\
$\sigma=\sqrt{\frac{\sum\left(\mathrm{f}_{\text {exp }}-\mathrm{f}_{\mathrm{cal}}\right)^{2}}{\mathrm{~N}}}$ & & & &
\end{tabular}

En el capítulo 2.4 de esta memoria se estableció el marco de referencia por el cual las propiedades volumétricas y dieléctricas estaban relacionadas mediante la 
ecuación de Clausius Mossotti cuando se hace referencia a fluidos no polares y a la ecuación de Debye para fluidos polares, y tal como se estableció al principio de este apartado los valores experimentales de frecuencia de resonancia nos permitirán validar la teoría de las perturbaciones descrita con detalle en el apartado 3.4 .

Determinando los valores de permitividad dieléctrica de la fase líquida y gaseosa, recurriendo al método establecido por Harvey y Lemmon [21], podremos calcular la constante dieléctrica de la sustancia a partir de los valores de densidad, dicha correlación que se encuentra descrita en el apartado 2.4, nos permite obtener los valores de densidad de polarización y por lo tanto los valores de la constante dieléctrica. Los valores de densidad del $\mathrm{CO}_{2}$ se han obtenido con la ecuación de estado Span \& Wagner [16] implementada en el software NIST REFPROP [20], y los valores de temperatura leídos con el medidor, obteniendo los valores de las constantes dieléctricas del líquido y de vapor saturado mediante las ecuaciones 2.41 a 2.44 .

La tabla 5.7 muestra los valores obtenidos de las densidades de polarización $(\mathrm{P} / \mathrm{\rho})$ del líquido y del vapor saturado y los valores de las contantes dieléctricas calculadas por medio del método establecido por Harvey y Lemmon [21]. 
Tabla 5.7 Valores de densidades de polarización eléctrica $\mathrm{P} / \rho$ y valores de constante dieléctrica $(\varepsilon)$ obtenidos por medio del método establecido por Harvey y Lemmon [21].

\begin{tabular}{|c|c|c|c|c|}
\hline$T_{\exp } / K$ & P/p liq & $\mathrm{P} / \rho$ gas & E liq & $\varepsilon$ gas \\
\hline 273.35 & 7.655 & 7.488 & 1.576 & 1.051 \\
\hline 278.36 & 7.661 & 7.503 & 1.553 & 1.060 \\
\hline 283.35 & 7.667 & 7.519 & 1.528 & 1.071 \\
\hline 288.35 & 7.672 & 7.538 & 1.500 & 1.086 \\
\hline 293.36 & 7.677 & 7.560 & 1.466 & 1.104 \\
\hline 298.35 & 7.680 & 7.587 & 1.423 & 1.132 \\
\hline 300.35 & 7.681 & 7.601 & 1.399 & 1.149 \\
\hline 302.35 & 7.681 & 7.620 & 1.366 & 1.175 \\
\hline 303.36 & 7.679 & 7.634 & 1.340 & 1.197 \\
\hline 303.66 & 7.678 & 7.640 & 1.328 & 1.207 \\
\hline 303.83 & 7.677 & 7.645 & 1.319 & 1.216 \\
\hline 304.04 & 7.674 & 7.653 & 1.301 & 1.233 \\
\hline
\end{tabular}

Aplicando el método de las perturbaciones descrito en el apartado 3.4 se ha podido dar validez a la técnica experimental descrita en esta tesis doctoral, este método como ya se mencionó, relaciona las frecuencias de resonancia con la constante dieléctrica de la muestra, en esta tesis doctoral nos referimos al $\mathrm{CO}_{2}$. Aplicando la ecuación 3.35 dado que se han determinado los valores de la constante dieléctrica y que la relación $\mathrm{V}_{\mathrm{S}} / \mathrm{V}_{\mathrm{c}}$ se pude determinar a partir de la geometría de la cavidad, es posible obtener el parámetro " $\mathrm{A}$ " de manera analítica.

La figura 5.7 muestra los resultados obtenidos al aplicar la ecuación 3.35. Este parámetro de proporcionalidad nos devuelve la relación entre la frecuencia de resonancia y la constante dieléctrica (que depende de la presión y la temperatura), por lo que al aproximarse al punto crítico las frecuencias de resonancia se igualen haciendo que este parámetro tienda a cero. 


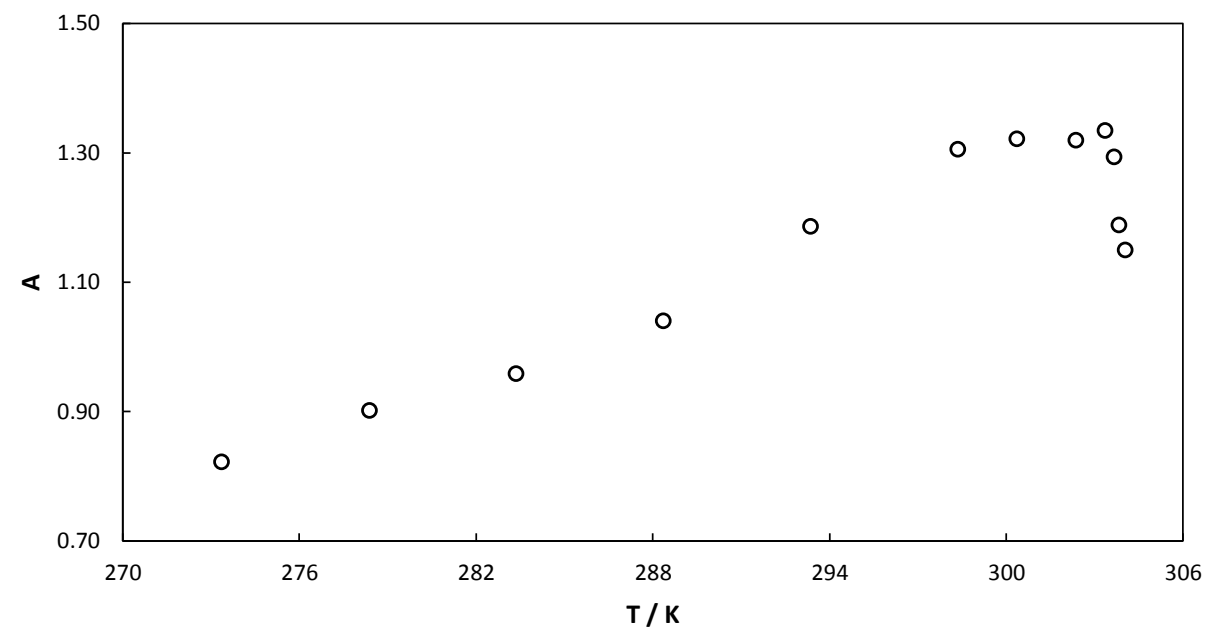

Figura 5.7 Parámetro A (o) frente a la temperatura obtenido al aplicar la ecuación 3.35 mediante el método de las perturbaciones.

\subsection{Análisis de los resultados}

En el presente apartado se han determinado los valores de equilibrio líquido vapor del $\mathrm{CO}_{2}$ mediante la determinación de las presiones de saturación en el rango de isotermas hasta las inmediaciones del punto crítico, a partir de los valores de las frecuencias de resonancia en cada uno de los puntos correspondientes. Los valores experimentales se han comparado con la ecuación de estado Span \& Wagner [16] implementada en el software NIST REFPROP [20], y con la literatura encontrada Kim et al. [72], Saleh et al. [73], y Gómez, [15]. Se han obtenido desviaciones promedio inferiores al $0.07 \%$ valores que son aceptables al compararlos con la literatura y que además se encuentran por debajo de la incertidumbre del equipo experimental de $0.010 \mathrm{MPa}$ siendo $0.29 \%$ su límite inferior de $3.502 \mathrm{MPa}$ y $0.14 \%$ su límite superior de $7.077 \mathrm{MPa}$.

Un estudio de las incertidumbres expandidas, da como resultado unos buenos resultados incluso en la determinación teórica del punto crítico lo que hace suponer que el procedimiento llevado a cabo para esta determinación ha sido el 
adecuado. Como cabía esperar las fuentes que más contribuyen son las debidas al polinomio de ajuste empleado para la determinación teórica del punto crítico, y la debida a la calibración de los instrumentos de medida empleados.

Se ha determinado el valor del punto crítico de mediante la comparación de las frecuencias de resonancia frente a la temperatura y a la presión, comparando los resultados obtenidos con los recogidos en la literatura Gómez, [15], Gil et al. [74], Suehiro et al. [75] y Diefenbacher et al. [76], Obteniéndose resultados satisfactorios y coherentes con la incertidumbre.

Se han determinado las constantes dieléctricas en cada uno de los puntos experimentales mediante el procedimiento descrito por Harvey y Lemmon [21], y se ha calculado el parámetro de proporcionalidad " $A$ " empleando para ello el método de las perturbaciones obteniendo la relación existente entre la frecuencia de resonancia y la constante dieléctrica. 
CAPítulo 6

\section{Envolvente de fases $y$}

\section{modelización de sistemas}

binarios con $\mathrm{CO}_{2}$ 



\subsection{Introducción}

En el capítulo anterior se estableció el principio de validación de la técnica experimental propuesta en esta tesis doctoral, una vez que se ha corroborado su correcto funcionamiento y se ha comprobado la calidad de los resultados obtenidos se han obtenido las desviaciones de los datos experimentales y se han comparándolos con la literatura, junto con un estudio del cálculo de su incertidumbre. En este apartado se muestran las medidas experimentales de las envolventes de fases de mezclas binarias que contiene $\mathrm{CO}_{2}$.

Las mezclas que se han estudiado tienen relación con el propósito de esta tesis doctoral y es contribuir con datos de calidad con el fin de optimizar los procesos en los que interviene la captura y almacenaje de $\mathrm{CO}_{2}$ (CCS). Para ello se han estudiado seis mezclas binarias formadas por $\mathrm{CH}_{4}, \mathrm{C}_{3} \mathrm{H}_{8}$ y $\mathrm{N}_{2}$ a diferentes composiciones de $\mathrm{CO}_{2}$. Todas estas mezclas han sido estudiadas en el rango de temperatura dentro de los límites del baño termostático, que establece como límite inferior $233.15 \mathrm{~K}$, llegando hasta la zona de máxima temperatura de cada uno de los sistemas (cricondentherm); las medidas experimentales también se han realizado en las proximidades al punto crítico y en las zonas de condensación retrógrada. Tal y como se describe en el apartado 6.2, las muestras fueron preparadas por el Centro Español de Metrología (CEM) fueron suministradas a una presión adecuada para evitar la separación por densidad de cualquiera de los compuestos.

Los datos experimentales obtenidos se compararán en primer lugar con los calculados con la ecuación de estado GERG 2008 [4], además se estudiará el comportamiento de las mezclas mediante la ecuación de estado cúbica de PengRobinson [17] empleando las reglas de mezcla de Wong-Sandler [18], donde se obtendrán los parámetros de ajuste de la modelización de los datos experimentales. 


\subsection{Preparación gravimétrica de las muestras gaseosas}

Las mezclas investigadas en esta tesis doctoral han sido preparadas en el Centro Español de Metrología (CEM) de acuerdo al procedimiento establecido CEM-PT0121, conforme a la norma ISO 6142-1. Las muestras empleadas fueron recibidas con sus certificados de calibración correspondientes donde se suministra toda la información necesaria sobre su pureza y sus correspondientes incertidumbres, la tabla 6.1 muestra toda la información detallada sobre la pureza en función de su fracción molar y la incertidumbre de estas muestras.

Tabla 6.1 Composición gravimétrica (fracción molar) e incertidumbre expandida en la composición $\mathrm{U}(\mathrm{x})$ con ( $\mathrm{k}=2$ ) de las mezclas investigadas.

\begin{tabular}{c|c|c|c}
\multicolumn{2}{c|}{ Mezcla } & \multicolumn{2}{c}{ Fracción molar (mol /mol) $10^{+2}$} \\
\hline $\mathrm{x}_{1}$ & $\left(1-\mathrm{x}_{1}\right)$ & $\mathrm{x}_{1}$ & $\left(1-\mathrm{x}_{1}\right)$ \\
\hline \multirow{2}{*}{$\mathrm{CO}_{2}$} & $\mathrm{CH}_{4}$ & $40.064 \pm 0.013$ & $59.936 \pm 0.002$ \\
& & $60.162 \pm 0.015$ & $39.837 \pm 0.004$ \\
\hline \multirow{2}{*}{$\mathrm{CO}_{2}$} & $\mathrm{C}_{3} \mathrm{H}_{\mathbf{8}}$ & $60.053 \pm 0.029$ & $39.947 \pm 0.021$ \\
& & $79.777 \pm 0.015$ & $20.223 \pm 0.011$ \\
\hline \multirow{2}{*}{$\mathrm{CO}_{2}$} & $\mathrm{~N}_{2}$ & $49.905 \pm 0.007$ & $50.095 \pm 0.003$ \\
& & $79.777 \pm 0.005$ & $20.222 \pm 0.012$
\end{tabular}

La tabla 6.2 muestra la información de los proveedores que han suministrado los gases en estado puro, en todas las muestras estudiadas en esta tesis doctoral la incertidumbre expandida esta expresada para un factor de cobertura $k=2$, el cálculo de esta incertidumbre está basado en las recomendaciones de la guía para la expresión de la incertidumbre [19]. 
Tabla 6.2 Lista de proveedores y pureza las sustancias empleadas para la elaboración de las mezclas estudiadas en esta tesis doctoral.

\begin{tabular}{c|cc} 
Sustancia & Proveedor & Pureza (\%) \\
\hline $\mathbf{C O}_{2}$ & Air Products & 99.995 \\
\hline $\mathbf{C H}_{4}$ & Linde AG & 99.995 \\
\hline $\mathbf{C}_{3} \mathbf{H}_{\mathbf{8}}$ & Carburos Metálicos & 99.993 \\
\hline $\mathbf{N}_{\mathbf{2}}$ & Linde AG & 99.999
\end{tabular}

\subsection{Determinación experimental de la envolvente de fases}

Al tratarse de mezclas de fluidos puede suceder que uno de los componentes se separe de la mezcla por densidad en la botella, para evitar este suceso, ya que podría alterar las medidas, antes de conectar botella que contiene la muestra en el equipo, se somete a un proceso de mezclado mediante rotación constante, este proceso se realiza a temperatura ambiente y durante un tiempo no inferior a 30 minutos. Con esto nos aseguramos que el contenido de la botella sea homogéneo en el momento de llenar las bombas de jeringa.

Mediante un procedimiento muy similar al descrito en el apartado 5.2 se han determinado los valores experimentales de los puntos de burbuja y de rocío en cada uno de los sistemas binarios estudiados en esta tesis doctoral, se han respetado los tiempos de vacío para la limpieza del equipo y los tiempos de espera en la selección de cada una de las isotermas.

Una vez establecido el procedimiento de limpieza, se ha procedido al llenado de las bombas ISCO a una presión suficiente que permita realizar la medida en las condiciones en las que se establece cada una de las isotermas, a una presión inferior (punto de rocío) o superior (punto de burbuja) de al menos un $20 \%$ para cada isoterma seleccionada, además se realiza una espera de 30 minutos por cada punto obtenido para asegurarse que toda la muestra se encuentra en la nueva fase 
y no exista la posibilidad de fases metaestables que alteren la medida. El caudal que se ha programado en las bombas ISCO para realizar las medidas es de 0.05 $\mathrm{ml} / \mathrm{min}$; se ha establecido este caudal con el fin de asegurarnos que la aparición de la nueva fase ocurra de manera gradual y no abruptamente, provocando una perturbación en la medida demasiado elevada que haga que el VNA no pueda realizar las correcciones necesarias y genere un error informático.

\subsection{Determinación de las incertidumbres}

Al igual que en el apartado 5.2 de esta tesis doctoral, la medida de la incertidumbre expandida combinada, se han determinado acorde a la "Guía para la expresión de la incertidumbre de medida" (JCGM 106 2012) [19]. La estimación de la incertidumbre expandida para la temperatura U(T) en la medida de los puntos de rocío y burbuja se realizará mediante el empleo de la ecuación 5.1, es en la incertidumbre expandida en la medida de la presión $U(p)$ donde se requiere un estudio más detallado. Tal y como se comentó en el apartado 3.4.2.

"La discontinuidad de la constante dieléctrica debido a la aparición de una nueva fase se verá reflejada como una variación en la frecuencia de resonancia de la cavidad (ecuación 3.35). De igual manera, el cambio de presión en la aparición de una nueva fase a temperatura constante hace que la frecuencia de resonancia varíe debido al cambio en la densidad de la muestra. Esta variación es continua y lineal, por lo que se puede normalizar mediante un polinomio de segundo grado, descartando el efecto en la frecuencia, de la presión sobre la densidad de la fase quedando únicamente la discontinuidad provocada por la transición de la fase en la aparición de un punto de burbuja o de rocío."

La frecuencia de resonancia se normaliza con un polinomio de segundo grado. Este ajuste debe incluirse como una contribución en la incertidumbre expandida debida al polinomio de ajuste $\mathrm{u}\left(\mathrm{p}_{\mathrm{Pol}}\right)$ en sustitución del $\mathrm{u}\left(\mathrm{p}_{\text {lineal }}\right)$ en la ecuación 
5.3. Además, se debe de tener en cuenta la contribución debida la composición en la medida del punto de burbuja o rocío $\mathrm{u}(\mathrm{p}(\mathrm{x}))$. Esta contribución se ha determinado a partir de los certificados de calibración emitidos por el CEM. EI impacto de esta contribución en la determinación de la incertidumbre en el cambio de fase, se ha evaluado a partir del coeficiente de sensibilidad obtenido en la ecuación de estado para las mezclas estudiadas mediante el REFPROP [20].

Teniendo en cuentas estas consideraciones la incertidumbre expandida $k=2$ debida a la presión en la determinación de los puntos de burbuja y rocío en las mezclas estudiadas en esta tesis doctoral será.

$$
\begin{aligned}
\mathrm{U}(\mathrm{p})= & 2 \cdot\left[\mathrm{u}\left(\mathrm{p}_{\text {res }}\right)^{2}+\mathrm{u}\left(\mathrm{p}_{\text {rep }}\right)^{2}+\mathrm{u}\left(\mathrm{p}_{\text {cal }}\right)^{2}+\mathrm{u}\left(\mathrm{p}_{\text {Pol }}\right)^{2}\right. \\
& \left.+\mathrm{u}(\mathrm{p}(\mathrm{x}))^{2}\right]^{1 / 2}
\end{aligned}
$$

\subsection{Modelización de los datos experimentales}

Las ecuaciones de estado son empleadas con el fin de modelizar comportamientos complejos de fases y para el diseño de procesos. Recientemente se han desarrollado ecuaciones de estado multiparamétricas que han incrementado la precisión y el rango de aplicabilidad como es el caso de las ecuaciones de estado GERG-2008 [4], desarrollada para el gas natural y otras mezclas relacionadas empleada en esta tesis doctoral con el propósito de comparar los datos experimentales. Este capítulo presenta la importancia del empleo de las ecuaciones cúbicas de estado o (EoS) para el modelado y comportamiento del equilibrio líquido-vapor de mezclas para todos los grados de no idealidad.

En las ecuaciones de estado (EoS) las relaciones volumétricas entre la presión, el volumen molar y la temperatura absoluta juegan un importante papel en la 
modelización termodinámica del equilibrio líquido-vapor especialmente a elevadas presiones, y se han convertido en una poderosa herramienta para la correlación y predicción del equilibrio líquido vapor para mezclas no ideales en un amplio rango de presiones y temperaturas.

\subsubsection{La ecuación GERG - 2008}

La ecuación GERG-2008 se forma en términos de energía libre de Helmholtz reducida $\alpha$, con las variables independientes, densidad $(\rho)$ y temperatura $(T)$, dividiéndose en dos partes: una parte que representa las propiedades de mezcla del gas ideal $\left(\alpha^{0}\right)$ y otra $\left(\alpha^{\mathrm{r}}\right)$ que representa el comportamiento real de mezcla,

$$
\alpha(\delta, \tau, \overline{\mathrm{x}})=\alpha^{0}(\rho, \mathrm{T}, \overline{\mathrm{x}})+\alpha^{\mathrm{r}}(\delta, \tau, \overline{\mathrm{x}})
$$

donde $\alpha^{0}$ representa las propiedades de la mezcla del gas ideal a una densidad $\rho$, temperatura T y composición $\overline{\mathrm{x}}$ de mezcla dada de acuerdo a la ecuación 6.3.

$$
\alpha^{0}(\rho, T, \bar{x})=\sum_{i=1}^{N} x_{i}\left[\alpha_{o i}^{o}(\rho, T)+\ln x_{i}\right]
$$

La parte residual de la energía libre de Helmholtz $\alpha^{\mathrm{r}}$ de la mezcla viene dada por la ecuación 6.5,

$$
\alpha^{\mathrm{r}}(\delta, \tau, \overline{\mathrm{x}})=\sum_{\mathrm{i}=1}^{\mathrm{N}} \mathrm{x}_{\mathrm{i}} \alpha_{\mathrm{oi}}^{\mathrm{r}}(\delta, \tau)+\Delta \alpha_{\mathrm{ij}}^{\mathrm{r}}(\delta, \tau, \overline{\mathrm{x}})
$$

donde $\delta$ es la densidad reducida de mezcla y $\tau$ es la inversa de la temperatura de mezcla de acuerdo a la ecuación 6.5. 


$$
\begin{aligned}
\delta & =\frac{\rho}{\rho_{\mathrm{r}}(\overline{\mathrm{x}})} \\
\tau & =\frac{\mathrm{T}_{\mathrm{r}}(\overline{\mathrm{x}})}{\mathrm{T}}
\end{aligned}
$$

La ecuación 6.5 tiene en cuenta el comportamiento residual de la mezcla a las variables reducidas $\delta$ y $\tau$. El primer sumatorio en esta ecuación corresponde a la contribución lineal de la energía libre residual de Helmholtz de la EoS de la sustancia pura multiplicada por las fracciones molares $x_{i}$. El segundo término es la función de desviación $\Delta \alpha^{\mathrm{r}}(\delta, \tau, \overline{\mathrm{x}})$ que es la suma de todas las funciones de partida binarias específicas y generalizadas $\Delta \alpha_{\mathrm{ij}}^{\mathrm{r}}(\delta, \tau, \overline{\mathrm{x}})$ desarrolladas para las respectivas mezclas binarias. En la ecuación 6.4, la forma adimensional de la energía libre de Helmholtz en el estado de gas ideal del componente i está dado por la ecuación 6.7,

$$
\Delta \alpha^{\mathrm{T}}(\delta, \tau, \overline{\mathrm{x}})=\sum_{\mathrm{i}=1}^{\mathrm{N}} \sum_{\mathrm{j}=\mathrm{i}+1}^{\mathrm{N}} \mathrm{x}_{\mathrm{i}} \mathrm{x}_{\mathrm{j}} \mathrm{F}_{\mathrm{ij}} \alpha_{\mathrm{ij}}^{\mathrm{r}}(\delta, \tau)
$$

El parámetro $F_{i j}$ en la ecuación 6.5 se fija en $F_{i j}=1$, para las mezclas estudiadas como son la formada por $\mathrm{CH}_{4}+\mathrm{CO}_{2}$ y por $\mathrm{N}_{2}+\mathrm{CO}_{2}$, que tienen funciones de desviación específica. Si $\mathrm{F}_{\mathrm{ij}}=0$, quiere decir que la función de desviación de la mezcla binaria no está desarrollada como es el caso de la mezcla formada por $\mathrm{C}_{3} \mathrm{H}_{8}+\mathrm{CO}_{2}$.

En esta tesis doctoral la ecuación de estado GERG-2008, se ha empleado con la configuración establecida por defecto mediante el correspondiente programa de cálculo de propiedades termodinámicas REFPROP [20]. 


\subsubsection{La ecuación de Peng \& Robinson}

La ecuación de Peng-Robinson [17] surge del intento de mejorar la descripción en el área de la región crítica y a mejorar la precisión en la predicción de la densidad del líquido.

$$
\mathrm{p}=\frac{\mathrm{RT}}{\mathrm{V}-\mathrm{b}}-\frac{\mathrm{a}(\mathrm{T})}{\mathrm{V}(\mathrm{V}+\mathrm{b})+\mathrm{b}(\mathrm{V}-\mathrm{b})}
$$

Donde el parámetro a, es considerado como una medida de las fuerzas de atracción intermolecular y el término b, es conocido como el volumen de Van der Waals, covolumen o volumen excluido, y está relacionado con el tamaño de la molécula.

La ecuación 6.7 puede expresarse de forma cúbica de la siguiente manera,

$$
Z^{3}-(1-B) Z^{2}+\left(A-3 B^{2}-2 B\right) Z-\left(A B-B^{2}-B^{3}\right)=0
$$

Donde.

$$
\begin{gathered}
A=\frac{a p}{R^{2} T^{2}} \\
B=\frac{b p}{R T} \\
Z=\frac{p V}{R T}
\end{gathered}
$$

Los valores de $a$ y $b$, pueden obtenerse como función de las propiedades críticas tales como la temperatura crítica $\left(T_{c}\right)$ y presión crítica $\left(p_{c}\right)$ de la primera y segunda derivada de la presión con respecto al volumen a temperatura constante. 


$$
\begin{aligned}
& \mathrm{a}\left(\mathrm{T}_{\mathrm{C}}\right)=0.45724 \frac{\mathrm{R}^{2} \mathrm{~T}_{\mathrm{C}}^{2}}{\mathrm{p}_{\mathrm{C}}} \\
& \mathrm{b}\left(\mathrm{T}_{\mathrm{C}}\right)=0.07780 \frac{\mathrm{RT}_{\mathrm{C}}}{\mathrm{p}_{\mathrm{C}}}
\end{aligned}
$$

Mientras que, a temperaturas distintas a las críticas,

$$
\begin{gathered}
a(T)=a\left(T_{C}\right) \cdot \alpha\left(T_{r}, \omega\right) \\
b(T)=b\left(T_{C}\right)
\end{gathered}
$$

donde $\alpha\left(T_{r}, \omega\right)$ es una función adimensional de la temperatura reducida y el factor acéntrico $\omega$. Para las sustancias de estudio la relación entre $\alpha$ y $T_{r}$, puede ser linealizada mediante la siguiente expresión,

$$
\alpha=\left[1+\mathrm{k}\left(1-\sqrt{\mathrm{T}_{\mathrm{r}}}\right)\right]^{2}
$$

donde el valor de $\mathrm{k}$ es una constante característica de cada sustancia, siendo correlacionada a través del factor acéntrico cuya expresión resulta.

$$
\mathrm{k}=0.37464+1.54226 \omega-0.26992 \omega^{2}
$$

El comportamiento de una mezcla se ve afectado no solo por las interacciones entre moléculas iguales " $\mathrm{ii}$ ", sino también por interacciones "ij" entre las moléculas " $\mathrm{i}$ " y "j" que componen la mezcla. Para aplicar EoS a una mezcla, se necesitan conocer las interacciones entre los componentes de la mezcla. Uno de los métodos más empleados para indicar estas interacciones son las reglas de mezclado con parámetros de interacción específicos del sistema los cuales se emplean con el fin de extender la EoS hasta las mezclas de múltiples componentes. 
Wong \& Sandler [18], propusieron a una regla de mezclado similar a las reglas de mezclado dependientes de la composición, señalando que no hay una restricción teórica en unas reglas de mezclado dependientes de la temperatura, la composición o la densidad, aparte de que cualquiera de estas reglas debería das lugar a una dependencia cuadrática del segundo coeficiente de virial en la composición. La ventaja de una regla de regla de mezclado independiente de la densidad, en contraste con las dependientes de la densidad, es que se mantiene la naturaleza de la ecuación cúbica de estado, en consecuencia, desarrollaron una nueva regla de mezclado basada en el uso de energía libre de Helmholtz en exceso $\left(A^{E}\right)$ en lugar de energía de $G i b b s$ de exceso $\left(G^{E}\right)$.

El uso de las energías de exceso de Helmholtz de mezcla preferible, cuando la energía de Gibbs de exceso se puede aproximar como:

$$
\mathrm{G}^{\mathrm{E}}(\mathrm{T}, \mathrm{x}, \mathrm{p}=\mathrm{low}) \approx \mathrm{A}^{\mathrm{E}}(\mathrm{T}, \mathrm{x}, \mathrm{p}=\mathrm{low}) \approx \mathrm{A}^{\mathrm{E}}(\mathrm{T}, \mathrm{x}, \mathrm{p}=\infty)
$$

La dependencia cuadrática con la composición del segundo coeficiente de virial se consigue al igualar el coeficiente $B$ de la ecuación de virial a los parámetros a y b de la ecuación cúbica de estado de la siguiente manera:

$$
\mathrm{B}(\mathrm{T})=\mathrm{b}-\frac{\mathrm{a}}{\mathrm{RT}}
$$

Dado que la dependencia de la composición del segundo coeficiente de virial es cuadrática, la expresión para las reglas de mezcla es:

$$
a_{m}=b_{m}\left(\sum_{i}^{N} x_{i} \frac{a_{i}}{b_{i}}+\frac{A_{\infty}^{E}}{C}\right)
$$




$$
\mathrm{b}_{\mathrm{m}}=\frac{\sum \sum \mathrm{x}_{\mathrm{i}} \mathrm{x}_{\mathrm{j}}(\mathrm{b}-\mathrm{a} / \mathrm{RT})_{\mathrm{ij}}}{1-\mathrm{A}_{\infty}^{\mathrm{E}} / \mathrm{CRT}^{-}-\sum_{\mathrm{i}=1}^{\mathrm{N}} \mathrm{x}_{\mathrm{i}} \mathrm{a}_{\mathrm{i}} / \mathrm{b}_{\mathrm{i}} \mathrm{RT}}
$$

El término $(b-a / R T)_{i j}$ es el segundo coeficiente de virial cruzado independiente de la composición de la EoS, que se relaciona con los parámetros de los componentes puros $a_{i}$ y $b_{i}$ de la siguiente manera,

$$
\left(b-\frac{a}{R T}\right)_{i j}=\frac{\left[\left(b_{i}-\frac{a_{i}}{R T}\right)+\left(b_{j}-\frac{a_{j}}{R T}\right)\right]}{2}\left(1-k_{i j}\right)
$$

donde $k_{i j}$ es un parámetro de iteración binaria del segundo coeficiente de virial.y donde $\mathrm{C}$ es una constante numérica, dependiente de la EoS empleada, que para PREoS es $C=\operatorname{Ln}(\sqrt{2}-1) / \sqrt{2}$.

Empleando el modelo de NRTL [77] para el cálculo de $\mathrm{A}_{\infty}^{\mathrm{E}} /(\mathrm{RT})$,

$$
\frac{A_{\infty}^{E}}{C R T}=\sum_{i} x_{i}\left(\frac{\sum_{j} x_{j} g_{i j} \tau_{j i}}{\sum_{k} x_{k} g_{i j}}\right)
$$

Con $\mathrm{g}_{\mathrm{ij}}=\exp \left(-\alpha_{\mathrm{ij}} \tau_{\mathrm{ji}}\right)$, donde el parámetro $\alpha_{\mathrm{ij}}=\alpha_{\mathrm{ji}}$ corresponde al parámetro no aleatorio NRTL [77]. Existen por lo tanto cuatro parámetros ajustables $\alpha, \tau_{\mathrm{ji}}, \tau_{\mathrm{ij}}, \mathrm{k}_{\mathrm{ij}}$ en esta versión de las reglas de mezcla de Wong \& Sandler, pudiendo ser usados como parámetros correlacionables como parámetros ajustables.

El método de punto de burbuja y punto de rocío se ha empleado en la modelización de los datos experimentales aplicando el cálculo de la fugacidad mediante la ecuación de estado propuesta al principio de este apartado. Para la modelización se emplearon como datos de partida la composición de la mezcla y 
la temperatura de manera que se calcularon las presiones a cada una de las isotermas estudiadas. Se ha utilizado un procedimiento de regresión múltiple nolineal, desarrollado por Nelder \& Mead [78] y considerando una modificación de la función objetivo (ecuaciones 6.19 y 2.20), obteniendo el mínimo error según la ecuación. Se empleó una serie de modificaciones propias del programa desarrollado en Matlab por Martín et al. [79] al final de este capítulo, se muestra una tabla con los resultados de las modelizaciones de los sistemas estudiados en esta tesis doctoral.

$$
\begin{gathered}
\text { Punto de burbuja (FO) }=\sum_{1}^{\mathrm{N}}\left(\mathrm{p}_{\exp }-\mathrm{p}_{\text {cal }}\right)_{\mathrm{i}}^{2}+\sum_{1}^{\mathrm{N}}\left(\mathrm{y}_{\exp }-\mathrm{y}_{\text {cal }}\right)_{\mathrm{i}}^{2} \\
\text { Punto de rocío (FO) }=\sum_{1}^{\mathrm{N}}\left(\mathrm{p}_{\exp }-\mathrm{p}_{\text {cal }}\right)_{\mathrm{i}}^{2}+\sum_{1}^{\mathrm{N}}\left(\mathrm{x}_{\exp }-\mathrm{x}_{\text {cal }}\right)_{\mathrm{i}}^{2}
\end{gathered}
$$

\subsection{Resultados experimentales}

Para la elaboración de esta tesis doctoral se han realizado las medidas de seis sistemas binarios a diferentes composiciones de $\mathrm{CO}_{2}$ tal y como se ha descrito al principio de este capítulo, mediante diferentes isotermas en el rango de temperaturas desde $233.15 \mathrm{~K}$ hasta el punto de máxima temperatura (cricondentherm) de cada uno de los sistemas.

La figura 6.1, muestra un ejemplo de cómo la normalización de la frecuencia en función de la presión permite obtener de manera gráfica el cambio de fase, dado que cuando se representa la frecuencia de resonancia frente a la presión los efectos del cambio de fase no se hacen visibles, dado que, la variación en la frecuencia de resonancia es continua y se puede normalizar mediante un polinomio de segundo grado, descartando el efecto en la frecuencia, de la presión sobre la densidad de la fase, quedando únicamente la discontinuidad provocada 
por la transición de la fase en la aparición de la nueva fase. Por tanto, una vez seleccionad de manera gráfica los intervalos que comprendan el cambio de fase, se ha procedido al ajuste de un polinomio de segundo grado con el fin de determinar más claramente el valor que debería corresponder al cambio de fase.

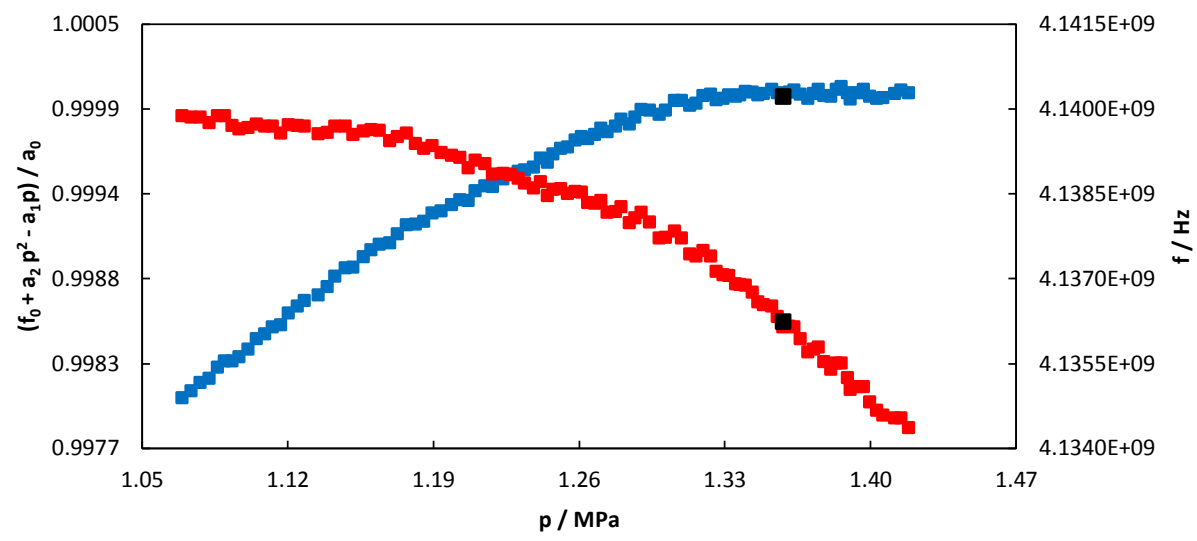

Figura 6.1Frecuencia normalizada ( $\square$ ) y la frecuencia de resonancia ( $\square$ ) en función de la presión, en la determinación experimental del punto de burbuja ( $\mathbf{(})$ para el sistema (0.6) $\mathrm{CO}_{2}+(0.4) \mathrm{C}_{3} \mathrm{H}_{8}$ a $\mathrm{T}=248.15 \mathrm{~K}$.

A continuación, se muestran los resultados experimentales de las envolventes de fase para cada uno de los sistemas estudiados en esta tesis doctoral, los puntos de burbuja y rocío fueron determinados con respecto a variaciones de la presión a lo largo de una serie de isotermas. Se compararon los resultados obtenidos con los calculados mediante la ecuación GERG 2008 [4] además de comparar las desviaciones obtenidas con la literatura existente, por último se ha empleado la ecuación cúbica de estado de Peng-Robinson [17] mediante las reglas de mezcla de Wong-Sandler [18] obteniendo los parámetros de ajuste de cada uno de los sistemas. 


\subsubsection{Sistema binario $(0.4) \mathrm{CO}_{2}+(0.6) \mathrm{CH}_{4}$}

En la tabla 6.3 se muestran los valores experimentales del sistema dióxido de carbono + metano a una composición de 0.40064 en fracción molar de dióxido de carbono.

Tabla 6.3 Datos experimentales de los puntos de burbuja, rocío y frecuencia de resonancia del sistema $(0.40064) \mathrm{CO}_{2}+(0.59936) \mathrm{CH}_{4}$ con una incertidumbre expandida $(\mathrm{k}=2)$ en $\mathrm{U}(\mathrm{T})=0.05 \mathrm{~K} ; \mathrm{U}(\mathrm{p})=0.010 \mathrm{MPa}$. Presiones calculadas mediante la ecuación de estado de GERG 2008 [4].

\begin{tabular}{|c|c|c|c|c|c|}
\hline Ensayo & $\mathbf{T}_{\text {exp }} / \mathrm{K}$ & $\mathrm{p}_{\mathrm{exp}} / \mathrm{MPa}$ & $f_{\text {exp }} / G H z$ & $\mathbf{p}_{\mathrm{GERG}} / \mathrm{MPa}$ & $\delta p \%$ \\
\hline Burbuja & 233.35 & 7.509 & 4.1374 & 7.579 & -0.92 \\
\hline Burbuja & 236.36 & 7.839 & 4.1376 & 7.831 & 0.10 \\
\hline Burbuja & 238.36 & 7.913 & 4.1377 & 7.977 & -0.80 \\
\hline Rocío* & 242.34 & 8.180 & 4.1379 & 8.185 & -0.07 \\
\hline Rocío* & 247.33 & 8.152 & 4.1382 & 8.143 & 0.12 \\
\hline Rocío* & 248.33 & 8.065 & 4.1383 & 8.030 & 0.43 \\
\hline Rocío* & 249.33 & 7.794 & 4.1385 & 7.786 & 0.10 \\
\hline Rocío* & 249.53 & 7.682 & 4.1386 & 7.692 & -0.13 \\
\hline Rocío* & 249.63 & 7.621 & 4.1386 & 7.629 & -0.10 \\
\hline Rocío* & 249.74 & 7.555 & 4.1386 & 7.529 & 0.35 \\
\hline Rocío* & 249.78 & 7.465 & 4.1387 & 7.472 & -0.10 \\
\hline Rocío & 249.78 & 7.159 & 4.1390 & 7.157 & 0.03 \\
\hline Rocío & 249.74 & 7.068 & 4.1390 & 7.090 & -0.32 \\
\hline Rocío & 249.63 & 6.945 & 4.1390 & 6.961 & -0.23 \\
\hline Rocío & 249.53 & 6.906 & 4.1391 & 6.871 & 0.52 \\
\hline Rocío & 249.32 & 6.721 & 4.1391 & 6.717 & 0.05 \\
\hline Rocío & 248.34 & 6.297 & 4.1395 & 6.218 & 1.26 \\
\hline Rocío & 247.33 & 5.913 & 4.1398 & 5.838 & 1.28 \\
\hline Rocío & 245.35 & 5.228 & 4.1402 & 5.244 & -0.30 \\
\hline Rocío & 242.34 & 4.450 & 4.1408 & 4.533 & -1.84 \\
\hline Rocío & 238.36 & 3.818 & 4.1414 & 3.785 & 0.87 \\
\hline Rocío & 236.36 & 3.465 & 4.1417 & 3.464 & 0.03 \\
\hline Rocío & 233.35 & 3.051 & 4.1421 & 3.036 & 0.51 \\
\hline
\end{tabular}


El sistema fue medido entre el rango de temperaturas de (233.15 a 249.15) K y en el rango de presiones de (3.0 a 8.2) MPa, incluida la zona retrógrada considerando el punto crítico de referencia calculado con la ecuación GERG-2008 [4], la incertidumbre expandida combinada para un factor de cobertura de $k=2$ en la medida de la temperatura fue de $U(T)=0.05 \mathrm{~K}$ y en la medida de la presión de $U(p)=0.010 \mathrm{MPa}$, mientras que la incertidumbre relativa en el rango de (3.051 a 8.177) $\mathrm{MPa}$ en la envolvente fue de $U_{r}(p)=0.33 \%(\mathrm{MPa} / \mathrm{MPa})$ y $U_{r}(p)=0.12 \%$ ( $\mathrm{MPa} / \mathrm{MPa})$ respectivamente. No se han observado la presencia de estados metaestables en el transcurso de las medidas, las desviaciones porcentuales se han calculado tomando como referencia los valores obtenidos con la ecuación GERG 2008 [4], resultando que la desviación media en la determinación del punto de burbuja ha sido del $0.61 \%$ mientras que la desviación porcentual promedio para la determinación del punto de rocío ha sido menor de $0.45 \%$, significativamente menor que los obtenidos en la determinación de los puntos de burbuja.

La figura 6.2 muestra la envolvente de fases obtenida experimentalmente, además en dicha figura se ha representado el resultado de la modelización mediante Peng-Robinson [17] empleando las reglas de mezcla de Wong-Sandler [18] así como la curva calculada por medio de la ecuación GERG 2008 [4], también se han incluido en la figura los valores experimentales de diferentes autores que han medido el equilibrio liquido vapor de este sistema. 


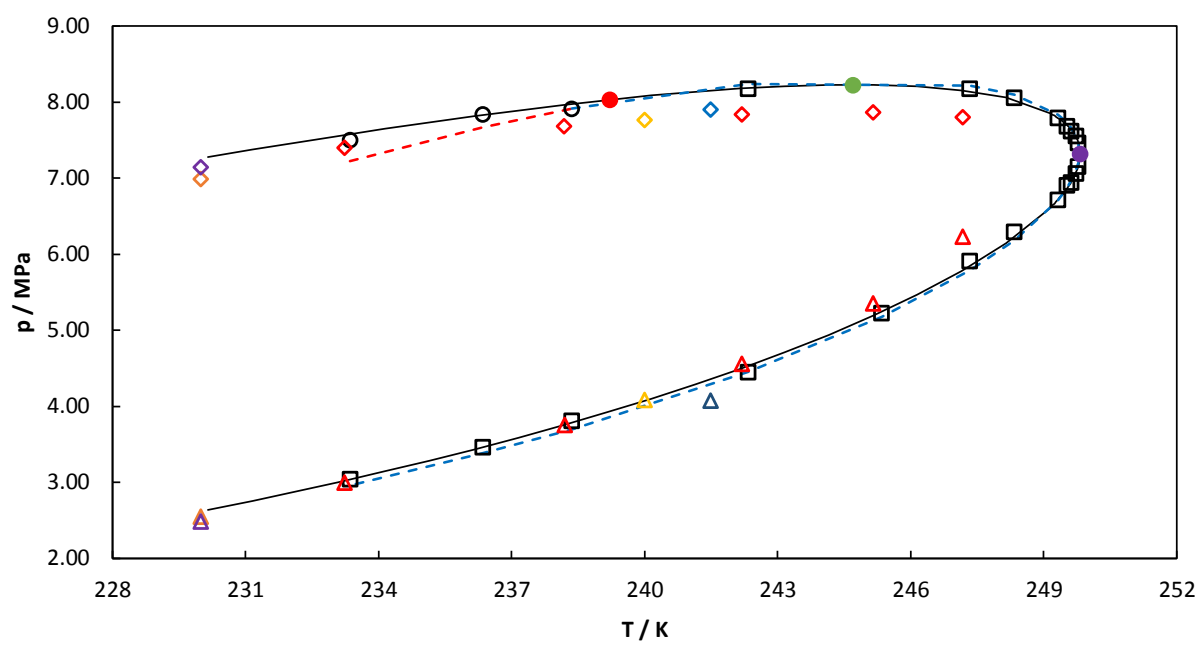

Figura 6.2Envolvente de fases para el sistema $\left(x_{1}\right) \mathrm{CO}_{2}+\left(1-\mathrm{x}_{1}\right) \mathrm{CH}_{4}\left(\mathrm{x}_{1}=0.40064\right)$ : punto de burbuja (o) rocío ( $\square$ ) esta tesis doctoral; curva calculada $(-)$, punto crítico $(\bullet)$, punto cricondentherm $(\bullet)$, y punto cricondenbar $(\bullet)$ GERG 2008 [4]; curva de ajuste de Peng-Robinson [17] \& Wong-Sandler [18] burbuja (- -) y rocío (- -); Donnelly et al. [80] burbuja $(\diamond)$, rocío $(\Delta)$; Wei et al. [81] burbuja $(\diamond)$, rocío $(\Delta)$; Webster et al. [82] burbuja $(\diamond)$, rocío $(\Delta)$; AlSahhaf et al. [83] burbuja $(\diamond)$, rocío $(\triangle)$ y Gómez. [15] burbuja $(\diamond)$, rocío $(\Delta)$.

Para esta mezcla se han encontrado diversos autores que, en el estudio del equilibrio, empleando para ello otras técnicas experimentales, han determinado un punto en sus sistemas que comparte características similares, en cuanto a composición; Donnelly et al. [80], Wei et al. [81], Webster et al. [82] y Al-Sahhaf et al. [83]. Gómez. [15], empleando una técnica similar en la se ha basado la presente tesis doctoral, ha determinado esta misma mezcla. Los valores obtenidos mediante esta nueva celda ofrecen una mejor repetibilidad con una menor incertidumbre que las obtenidas con la celda antecesora de Gómez [15].

En la figura 6.3 se han representado las desviaciones porcentuales respecto la ecuación GERG-2008 [4] frente a la temperatura de los datos experimentales y la literatura encontrada. 


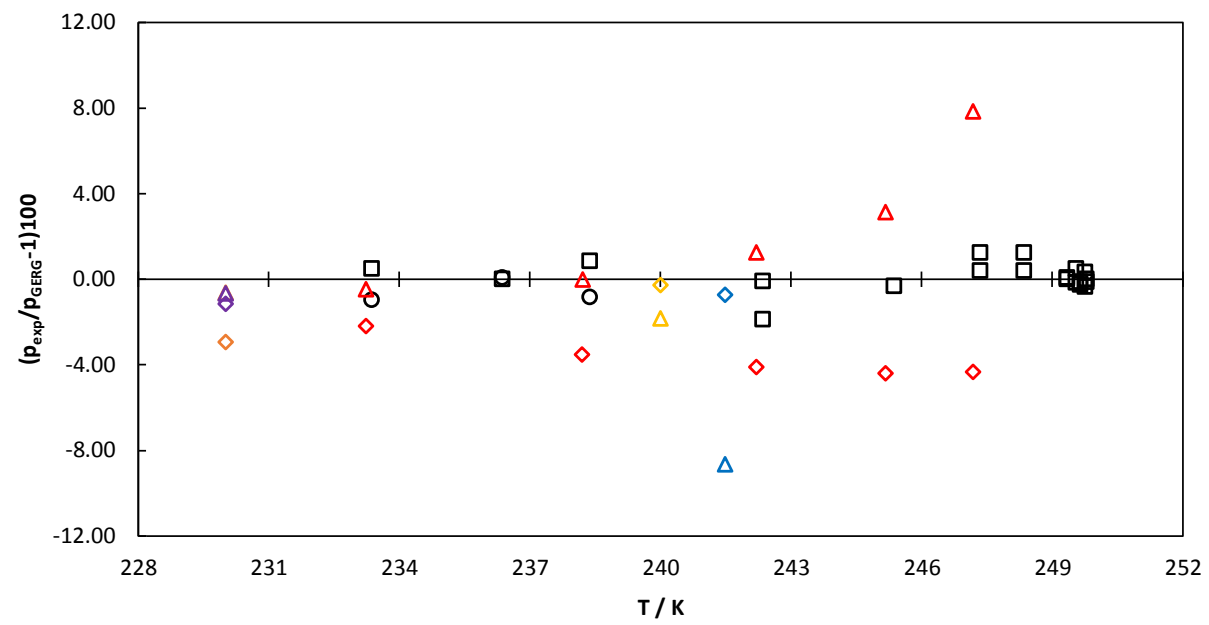

Figura 6.3Desviaciones relativas respecto la ecuación GERG-2008 [4] para el sistema $\left(\mathrm{x}_{1}\right) \mathrm{CO}_{2}+\left(1-\mathrm{x}_{1}\right) \mathrm{CH}_{4}$ $\left(x_{1}=0.40064\right)$; punto de burbuja (o) rocío $(\square)$ esta tesis doctoral; Donnelly et al. [80] burbuja $(\diamond)$, rocío $(\Delta)$; Wei et al. [81] burbuja $(\diamond)$, rocío $(\Delta)$; Webster et al. [82] burbuja $(\diamond)$, rocío $(\Delta)$; Al-Sahhaf et al. [83] burbuja $(\diamond)$, rocío $(\triangle)$ y Gómez. [15] burbuja ( () , rocío $(\Delta)$.

La figura muestra como las desviaciones porcentuales obtenidas en esta tesis doctoral no superan el $2 \%$ mientras que valores obtenidos por Gómez. [15], midiendo este mismo sistema, devuelve valores que se encuentran por encima del $4 \%$ y llegan hasta el $8 \%$ en el caso de la isoterma a $247.17 \mathrm{~K}$. La celda empleada por Gómez [15] no pudo determinar los puntos de rocío retrógrado en la isoterma $248.19 \mathrm{~K}$ mientas que este nuevo equipo en la isoterma $248.33 \mathrm{~K}$, muy cerca del valor dado por Gómez [15], si ha logrado determinar dicho valor con una desviación de $0.43 \%$.

Analizando una comparación de la ecuación GERG-2008 [4] con la literatura encontrada para los puntos que comparten una composición aproximada en $\mathrm{CO}_{2}$, Donnelly et al. [80] a una composición de 0.499 presenta una desviación media porcentual de $-0.72 \%$ en el punto burbuja, y con una composición de 0.395 devuelve una deviación del $-8.64 \%$ en el punto de rocío. La isoterma de $230.00 \mathrm{~K}$ de Wei et al. [81] a 0.419 de composición tiene una desviación de $-2.93 \%$ para el punto de burbuja, y para una composición de 0.407 la más cercana a la estudiada 
en esta tesis doctoral su desviación es de $-0.60 \%$ para el punto de rocío. Webster et al. [82], obtienen para el punto de burbuja una desviación del $-1.14 \%$ y para el punto de rocío una desviación del $0.64 \%$. Al-Sahhaf et al. [83], la desviación que obtienen respecto a la GERG 2008 [4] en el punto de burbuja es del $0.26 \%$, en el punto de rocío su desviación es del -1.83\% Para la isoterma $240.00 \mathrm{~K}$.

Los puntos que presenta mayor desviación son los devueltos por Donnelly et al. [80] -8.64\% y Gómez. [15] 7.86\% 


\subsubsection{Sistema binario (0.6) $\mathrm{CO}_{2}+(0.4) \mathrm{CH}_{4}$}

La tabla 6.4 muestra los datos experimentales junto con los calculados y sus deviaciones correspondientes del sistema dióxido de carbono + metano a una composición de 0.60162 en fracción molar de dióxido de carbono. A igual que el sistema visto en apartado anterior, la envolvente de fases para este nuevo sistema fue determinada entre el rango de temperaturas de (233.15 a 271.15 ) K y en el rango de presiones de (1.8 a 8.9) MPa incluida la zona retrógrada considerando el punto crítico de referencia calculado con GERG-2008 [4]. Al igual que en el sistema anteriormente descrito, no se ha observado la presencia de estados metaestables en el transcurso de las medidas.

Tabla 6.4 Datos experimentales de los puntos de burbuja, rocío y frecuencia de resonancia del sistema $(0.60162) \mathrm{CO}_{2}+(0.39837) \mathrm{CH}_{4}$ con una incertidumbre expandida $(\mathrm{k}=2)$ en $\mathrm{U}(\mathrm{T})=0.05 \mathrm{~K} ; \mathrm{U}(\mathrm{p})=0.010 \mathrm{MPa}$. Presiones calculadas mediante la ecuación de estado de GERG 2008 [4].

\begin{tabular}{l|ccccc} 
Ensayo & $\mathrm{T}_{\text {exp }} / \mathrm{K}$ & $\mathbf{p}_{\text {exp }} / \mathbf{M P a}$ & $\mathbf{f}_{\text {exp }} / \mathrm{GHz}$ & $\mathbf{p}_{\text {GERG }} / \mathrm{MPa}$ & $\delta \mathbf{p} \%$ \\
\hline Burbuja & 233.35 & 6.671 & 4.1355 & 6.667 & 0.07 \\
Burbuja & 243.34 & 7.528 & 4.1350 & 7.516 & 0.15 \\
Burbuja & 253.35 & 8.268 & 4.1345 & 8.327 & -0.71 \\
Burbuja & 258.34 & 8.657 & 4.1344 & 8.669 & -0.14 \\
Burbuja & 263.34 & 8.872 & 4.1345 & 8.909 & -0.41 \\
Rocío* & 266.32 & 8.896 & 4.1348 & 8.956 & -0.67 \\
Rocío* & 268.32 & 8.907 & 4.1352 & 8.899 & 0.10 \\
Rocío* & 269.34 & 8.775 & 4.1354 & 8.819 & -0.50 \\
Rocío* & 270.33 & 8.687 & 4.1355 & 8.673 & 0.17 \\
Rocío* & 270.82 & 8.454 & 4.1358 & 8.531 & -0.89 \\
Rocío* & 270.93 & 8.463 & 4.1358 & 8.481 & -0.22 \\
Rocío* & 271.03 & 8.355 & 4.1359 & 8.423 & -0.81 \\
Rocío* & 271.12 & 8.314 & 4.1359 & 8.350 & -0.43 \\
Rocío & 271.12 & 7.903 & 4.1362 & 7.961 & -0.73 \\
Rocío & 271.03 & 7.857 & 4.1362 & 7.863 & -0.07 \\
Rocío & 270.93 & 7.815 & 4.1363 & 7.779 & 0.47 \\
Rocío & 270.82 & 7.712 & 4.1363 & 7.700 & 0.16 \\
Rocío & 270.33 & 7.413 & 4.1366 & 7.428 & -0.20 \\
& & & & &
\end{tabular}


Tabla 6.3 Continuación.

\begin{tabular}{l|ccccc} 
Ensayo & $\mathbf{T}_{\text {exp }} / \mathbf{K}$ & $\mathbf{p}_{\text {exp }} / \mathbf{M P a}$ & $\mathbf{f}_{\text {exp }} / \mathbf{G H z}$ & $\mathbf{p}_{\text {GERG }} / \mathbf{M p a}$ & $\delta \mathbf{p} \%$ \\
\hline Rocío & 269.34 & 7.011 & 4.1368 & 7.023 & -0.17 \\
Rocío & 268.32 & 6.625 & 4.1371 & 6.688 & -0.94 \\
Rocío & 266.32 & 6.143 & 4.1377 & 6.140 & 0.05 \\
Rocío & 263.34 & 5.425 & 4.1381 & 5.469 & -0.80 \\
Rocío & 258.34 & 4.487 & 4.1389 & 4.555 & -1.48 \\
Rocío & 253.35 & 3.803 & 4.1397 & 3.812 & -0.22 \\
Rocío & 243.35 & 2.688 & 4.1411 & 2.656 & 1.17 \\
Rocío & 233.35 & 1.818 & 4.1424 & 1.818 & -0.01 \\
Sp \% $=\left(\frac{p_{\text {exp }}}{\mathrm{p}_{\text {GERG }}}-1\right) * 100$ & & & & \\
Punto de rocío retrógrado considerando el punto crítico calculado mediante la GERG 2008 [4].
\end{tabular}

Las incertidumbres expandidas combinadas obtenidas has sido determinadas con un factor de cobertura $\mathrm{k}=2$, dando como resultado una incertidumbre en la medida de la temperatura de $U(T)=0.05 \mathrm{~K}$ y en la medida de la presión de $U(p)=$ $0.010 \mathrm{MPa}$, mientras que la incertidumbre relativa en el rango de (1.818 a 8.907) MPa en la envolvente fue de $U_{r}(p)=0.55 \%(\mathrm{MPa} / \mathrm{MPa})$ y $U_{r}(p)=0.11 \%(\mathrm{MPa} / \mathrm{MPa})$ respectivamente.

La figura 6.4 muestra la figura de la envolvente de fases del sistema estudiado, en ella se muestra la curva de ajuste mediante la ecuación GERG 2008 [4] además de la modelización de estos datos experimentales por medio de la ecuación cúbica de estado de Peng-Robinson [17] mediante las reglas de mezcla de Wong-Sandler [18] obteniendo los parámetros de ajuste de cada uno de los sistemas los cuales están descritos al final de este capítulo. Al igual que en el sistema anteriormente estudiado, los datos experimentales han sido comparados, Donnelly et al. [80], Wei et al. [81], Webster et al. [82], Dávalos et al. [84], Al-Sahhaf et al. [83] y Gómez [15] 


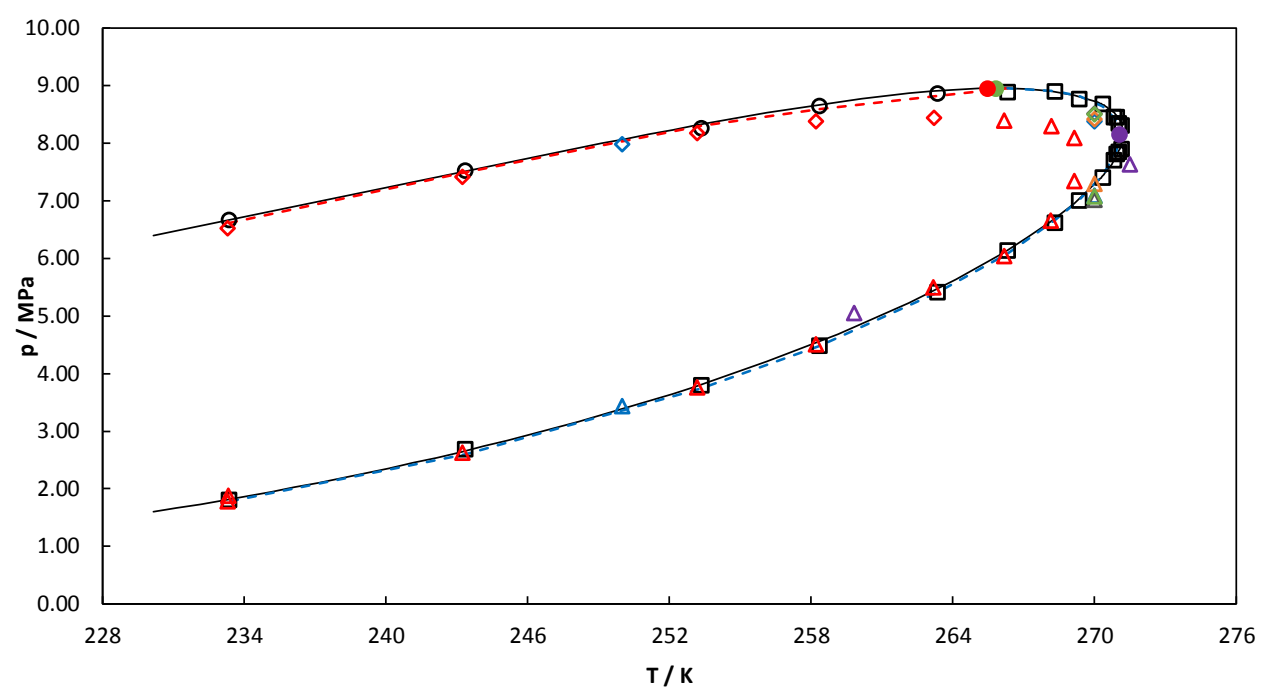

Figura 6.4Envolvente de fases para el sistema $\left(x_{1}\right) \mathrm{CO}_{2}+\left(1-\mathrm{x}_{1}\right) \mathrm{CH}_{4}\left(\mathrm{x}_{1}=0.60162\right)$; punto de burbuja (o) rocío ( $\square$ ) esta

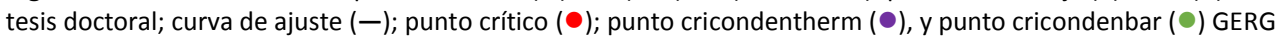
2008 [4]; curva de ajuste de Peng-Robinson [17] \& Wong-Sandler [18] burbuja ( - ) y rocío (--); Donnelly et al. [80] rocío $(\Delta)$; Wei et al. [81] burbuja $(\diamond)$, rocío $(\Delta)$; Webster et al. [82] burbuja $(\diamond)$, rocío ( $\Delta$ ); Dávalos et al. [84] burbuja $(\diamond)$, rocío $(\Delta)$; Al-Sahhaf et al. [83] burbuja $(\diamond)$, rocío $(\Delta)$ y Gómez [15] burbuja $(\diamond)$, rocío $(\Delta)$.

La mayoría de los datos de literatura encontrados para este sistema se sitúan en la zona de máxima temperatura (cricondentherm) que calculada con ecuación GERG 2008 [4] es de 271.20K, y Wei et al. [81], Webster et al. [82], Dávalos et al. [84] y Al-Sahhaf et al. [83] reportan datos a la isoterma de $270 \mathrm{~K}$.

En la figura 6.5, se muestran los resultados de las desviaciones porcentuales de los datos experimentales estudiados en esta tesis doctoral además de las desviaciones reportadas por la literatura, dichas desviaciones han sido comparadas en todos los casos con la ecuación de estado GERG 2008 [4]. Los resultados de esta mezcla presentan una desviación porcentual promedio de $0.30 \%$ para las medidas del punto de burbuja, mientras que para las medidas del punto de rocío se han obtenido unas desviaciones promedio de $0.49 \%$. 


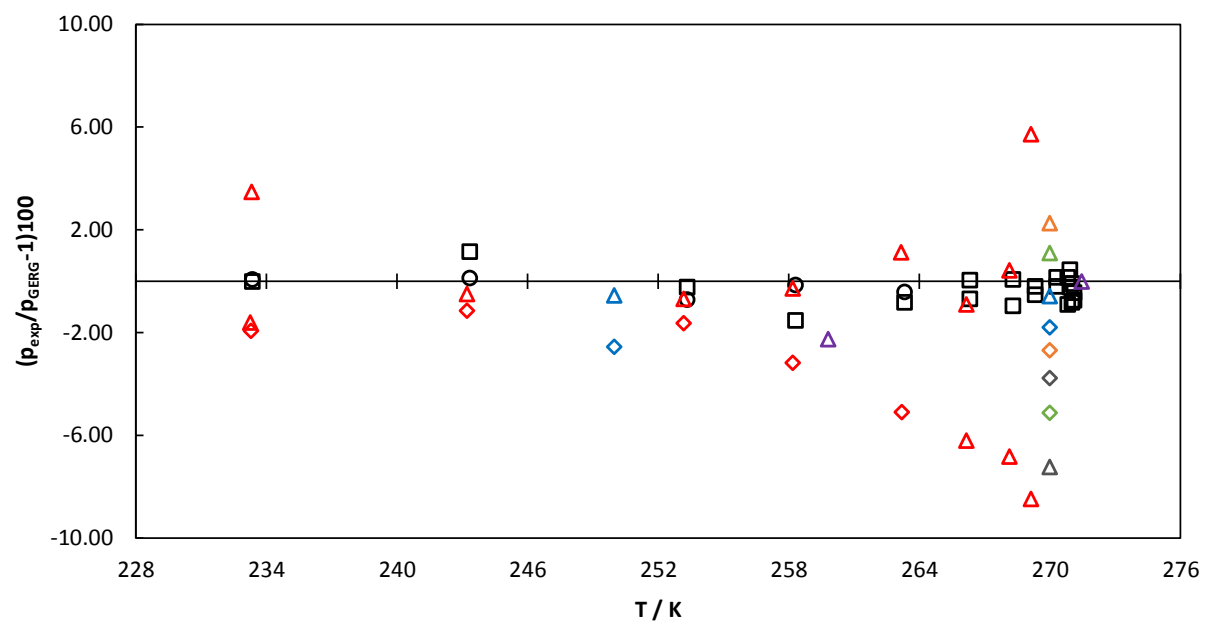

Figura 6.5Desviaciones relativas respecto la ecuación GERG-2008 [4] para el sistema $\left(\mathrm{x}_{1}\right) \mathrm{CO}_{2}+\left(1-\mathrm{x}_{1}\right) \mathrm{CH}_{4}$ $\left(x_{1}=0.60162\right)$; punto de burbuja (o) rocío ( $\square$ ) esta tesis doctoral; Donnelly et al. [80] rocío $(\Delta)$; Wei et al. [81] burbuja $(\diamond)$, rocío $(\Delta)$; Webster et al. [82] burbuja $(\diamond)$, rocío $(\Delta)$; Dávalos et al. [84] burbuja ( $\diamond)$, rocío $(\Delta)$; Al-Sahhaf et al. [83] burbuja $(\diamond)$, rocío $(\Delta)$ y Gómez [15] burbuja $(\diamond)$, rocío $(\Delta)$.

De los datos encontrados en la literatura Dávalos et al. [84] a la isoterma de 270 K y a la composición de 0.595 para el punto de rocío presenta una desviación porcentual del -7.22\%, Gómez [15] con su equipo previo a la isoterma $269.14 \mathrm{~K}$ cercana a la de Dávalos et al. [84] devuelve una incertidumbre del $-8.45 \%$ para el punto de rocío retrógrado y del 5.75\% para el punto de rocío, en esta tesis doctoral a la isoterma de $269.34 \mathrm{~K}$ la desviación obtenida ha sido de $-0.50 \%$.

Con un equipo previo al presentado en esta tesis doctoral, Gómez. [15] a determinado a la misma composición este sistema binario donde como en el sistema anterior, no se consiguió determinar puntos cercanos a la zona de temperatura máxima (cricondentherm) mientras que con la nueva celda que se presenta en esta tesis, si se han obtenido dichos datos experimentales con unas desviaciones inferiores al $0.4 \%$, los datos proporcionados por Gómez. [15] presentan desviaciones porcentuales en algunos casos del orden del $8 \%$ debido a problemas con la composición que impedían la repetibilidad, mientras que los presentados en esta tesis doctoral no superan el $1.5 \%$. 
Nos encontramos ante un nuevo equipo capaz de determinar de manera más precisa la envolvente de fases incluso en áreas donde antes se encontraban ciertas dificultades por lo que además de validar la técnica experimental con la determinación de la curva de vaporización del dióxido de carbono, se ha logrado mejorar de manera sustancial la determinación experimental de la envolvente de fases. 


\subsubsection{Sistema binario $(0.6) \mathrm{CO}_{2}+(0.4) \mathrm{C}_{3} \mathrm{H}_{8}$}

La tabla 6.5 muestra las medidas experimentales de la envolvente de fases del sistema dióxido de carbono + propano a la composición de 0.60053 en fracción molar de dióxido de carbono, esta tabla muestra las presiones en los puntos de burbuja y rocío para cada una de las isotermas estudiadas además de la frecuencia de resonancia asociada a cada presión y temperatura en el rango de temperaturas de (229.15 a 329.60) K y en el rango de presiones de (0.2 a 6.8) MPa incluida la zona retrógrada considerando el punto crítico de referencia calculado con la ecuación de estado GERG 2008 [4].

Tabla 6.5 Datos experimentales de los puntos de burbuja, rocío y frecuencia de resonancia del sistema $(0.60053) \mathrm{CO}_{2}+(0.39947) \mathrm{C}_{3} \mathrm{H}_{8}$ con una incertidumbre expandida $(\mathrm{k}=2) \mathrm{U}(\mathrm{T})=0.05 \mathrm{~K} ; \mathrm{U}(\mathrm{p})=0.010 \mathrm{MPa}$. Presiones calculadas mediante la ecuación de estado de GERG 2008 [4].

\begin{tabular}{l|ccccc} 
Ensayo & $\mathrm{T}_{\text {exp }} / \mathbf{K}$ & $\mathbf{p}_{\text {exp }} / \mathrm{MPa}$ & $\mathbf{f}_{\text {exp }} / \mathrm{GHz}$ & $\mathbf{p}_{\text {GERG }} / \mathrm{MPa}$ & $\delta \mathbf{p} \%$ \\
\hline Burbuja & 229.37 & 0.738 & 4.1400 & 0.738 & -0.10 \\
Burbuja & 233.35 & 0.845 & 4.1393 & 0.848 & 0.36 \\
Burbuja & 238.36 & 1.005 & 4.1381 & 1.002 & -0.33 \\
Burbuja & 243.35 & 1.180 & 4.1372 & 1.175 & -0.46 \\
Burbuja & 248.34 & 1.358 & 4.1360 & 1.367 & 0.68 \\
Burbuja & 253.35 & 1.594 & 4.1349 & 1.581 & -0.85 \\
Burbuja & 258.34 & 1.825 & 4.1339 & 1.815 & -0.56 \\
Burbuja & 263.34 & 2.070 & 4.1332 & 2.073 & 0.13 \\
Burbuja & 268.34 & 2.337 & 4.1326 & 2.353 & 0.67 \\
Burbuja & 273.34 & 2.640 & 4.1317 & 2.657 & 0.62 \\
Burbuja & 278.33 & 2.982 & 4.1309 & 2.984 & 0.07 \\
Burbuja & 283.38 & 3.342 & 4.1301 & 3.339 & -0.08 \\
Burbuja & 288.40 & 3.711 & 4.1292 & 3.716 & 0.15 \\
Burbuja & 293.41 & 4.128 & 4.1286 & 4.116 & -0.28 \\
Burbuja & 298.22 & 4.539 & 4.1281 & 4.521 & -0.40 \\
Burbuja & 303.36 & 4.966 & 4.1276 & 4.972 & 0.12 \\
Burbuja & 308.46 & 5.439 & 4.1272 & 5.435 & -0.09 \\
Burbuja & 313.47 & 5.915 & 4.1268 & 5.893 & -0.37 \\
Burbuja & 318.49 & 6.329 & 4.1267 & 6.338 & 0.14
\end{tabular}


Tabla 6.4 Continuación.

\begin{tabular}{|c|c|c|c|c|c|}
\hline Ensayo & $\mathrm{T}_{\exp } / \mathrm{K}$ & $\mathrm{p}_{\mathrm{exp}} / \mathrm{MPa}$ & $f_{\text {exp }} / G H z$ & $\mathbf{p}_{\text {GERG }} / \mathrm{Mpa}$ & $\delta \mathbf{p} \%$ \\
\hline Burbuja & 323.49 & 6.721 & 4.1268 & 6.719 & -0.03 \\
\hline Burbuja & 324.49 & 6.773 & 4.1270 & 6.778 & 0.08 \\
\hline Burbuja & 325.49 & 6.822 & 4.1272 & 6.827 & 0.07 \\
\hline Burbuja & 326.47 & 6.859 & 4.1272 & 6.861 & 0.03 \\
\hline Burbuja & 327.44 & 6.874 & 4.1274 & 6.873 & 0.00 \\
\hline Rocío* & 328.41 & 6.857 & 4.1277 & 6.861 & 0.06 \\
\hline Rocío* & 328.53 & 6.865 & 4.1278 & 6.856 & -0.14 \\
\hline Rocío* & 328.73 & 6.844 & 4.1279 & 6.844 & 0.00 \\
\hline Rocío* & 328.93 & 6.834 & 4.1279 & 6.827 & -0.10 \\
\hline Rocío* & 329.53 & 6.724 & 4.1281 & 6.702 & -0.32 \\
\hline Rocío* & 329.60 & 6.681 & 4.1281 & 6.655 & -0.39 \\
\hline Rocío & 329.60 & 6.538 & 4.1284 & 6.524 & -0.22 \\
\hline Rocío & 329.53 & 6.459 & 4.1285 & 6.459 & 0.01 \\
\hline Rocío & 328.93 & 6.201 & 4.1290 & 6.192 & -0.14 \\
\hline Rocío & 328.73 & 6.111 & 4.1291 & 6.127 & 0.26 \\
\hline Rocío & 328.53 & 6.056 & 4.1291 & 6.068 & 0.19 \\
\hline Rocío & 328.41 & 6.043 & 4.1291 & 6.034 & -0.14 \\
\hline Rocío & 327.44 & 5.807 & 4.1295 & 5.794 & -0.23 \\
\hline Rocío & 326.47 & 5.579 & 4.1302 & 5.589 & 0.18 \\
\hline Rocío & 325.49 & 5.394 & 4.1303 & 5.402 & 0.14 \\
\hline Rocío & 324.49 & 5.223 & 4.1305 & 5.227 & 0.08 \\
\hline Rocío & 323.49 & 5.068 & 4.1307 & 5.063 & -0.10 \\
\hline Rocío & 318.49 & 4.359 & 4.1313 & 4.362 & 0.06 \\
\hline Rocío & 313.47 & 3.783 & 4.1323 & 3.784 & 0.02 \\
\hline Rocío & 308.46 & 3.294 & 4.1330 & 3.292 & -0.05 \\
\hline Rocío & 303.36 & 2.867 & 4.1337 & 2.858 & -0.33 \\
\hline Rocío & 298.22 & 2.476 & 4.1344 & 2.475 & -0.05 \\
\hline Rocío & 293.41 & 2.158 & 4.1351 & 2.159 & 0.04 \\
\hline Rocío & 288.40 & 1.870 & 4.1358 & 1.868 & -0.13 \\
\hline Rocío & 283.38 & 1.599 & 4.1364 & 1.610 & 0.68 \\
\hline Rocío & 278.33 & 1.388 & 4.1370 & 1.381 & -0.53 \\
\hline Rocío & 273.34 & 1.176 & 4.1377 & 1.181 & 0.44 \\
\hline Rocío & 268.34 & 1.004 & 4.1383 & 1.005 & 0.05 \\
\hline Rocío & 263.34 & 0.846 & 4.1390 & 0.850 & 0.54 \\
\hline Rocío & 258.34 & 0.717 & 4.1397 & 0.715 & -0.19 \\
\hline
\end{tabular}


Tabla 6.4 Continuación

\begin{tabular}{|c|c|c|c|c|c|}
\hline Ensayo & $T_{\exp } / \mathrm{K}$ & $\mathrm{p}_{\mathrm{exp}} / \mathrm{MPa}$ & $f_{\text {exp }} / G H z$ & $\mathrm{p}_{\mathrm{GERG}} / \mathrm{Mpa}$ & $\delta p \%$ \\
\hline Rocío & 253.35 & 0.595 & 4.1402 & 0.598 & 0.60 \\
\hline Rocío & 248.34 & 0.496 & 4.1408 & 0.496 & -0.07 \\
\hline Rocío & 243.35 & 0.411 & 4.1415 & 0.409 & -0.58 \\
\hline Rocío & 238.36 & 0.334 & 4.1420 & 0.334 & 0.02 \\
\hline Rocío & 233.35 & 0.272 & 4.1428 & 0.270 & -0.67 \\
\hline Rocío & 229.37 & 0.227 & 4.1434 & 0.226 & -0.08 \\
\hline
\end{tabular}

Punto de rocío retrógrado considerando el punto crítico calculado mediante la GERG 2008 [4].

Los datos experimentales fueron comparados con la ecuación GERG 2008 [4], con un promedio de una desviación porcentual de $0.29 \%$ para las medidas del punto de burbuja, mientras que para las medidas del punto de rocío se han obtenido unas desviaciones promedio de $0.22 \%$. Hasta ahora este ha sido el sistema que menores desviaciones porcentuales con la ecuación GERG 2008 [4].

la incertidumbre expandida combinada para un factor de cobertura de $k=2$ en la medida de la temperatura fue de $\mathrm{U}(\mathrm{T})=0.05 \mathrm{~K}$ y en la medida de la presión de $U(p)=0.010 \mathrm{MPa}$, mientras que la incertidumbre relativa en el rango de (0.227 a 6.874) $\mathrm{MPa}$ en la envolvente fue de $U_{r}(p)=4.41 \%(\mathrm{MPa} / \mathrm{MPa})$ y $U_{r}(p)=0.15 \%$ $(\mathrm{MPa} / \mathrm{MPa})$ respectivamente. No se han observado la presencia de estados metaestables en el transcurso de las medidas.

La figura 6.6 muestra la envolvente de fases obtenida experimentalmente en la misma se ha representado el resultado de la modelización mediante la ecuación cúbica de estado de Peng-Robinson [17] empleando las reglas de mezcla de WongSandler [18] así como la curva de ajuste por medio de la ecuación GERG 2008 [4], además se han incluido los valores experimentales de diferentes autores que han medido el equilibrio liquido vapor de este sistema. 


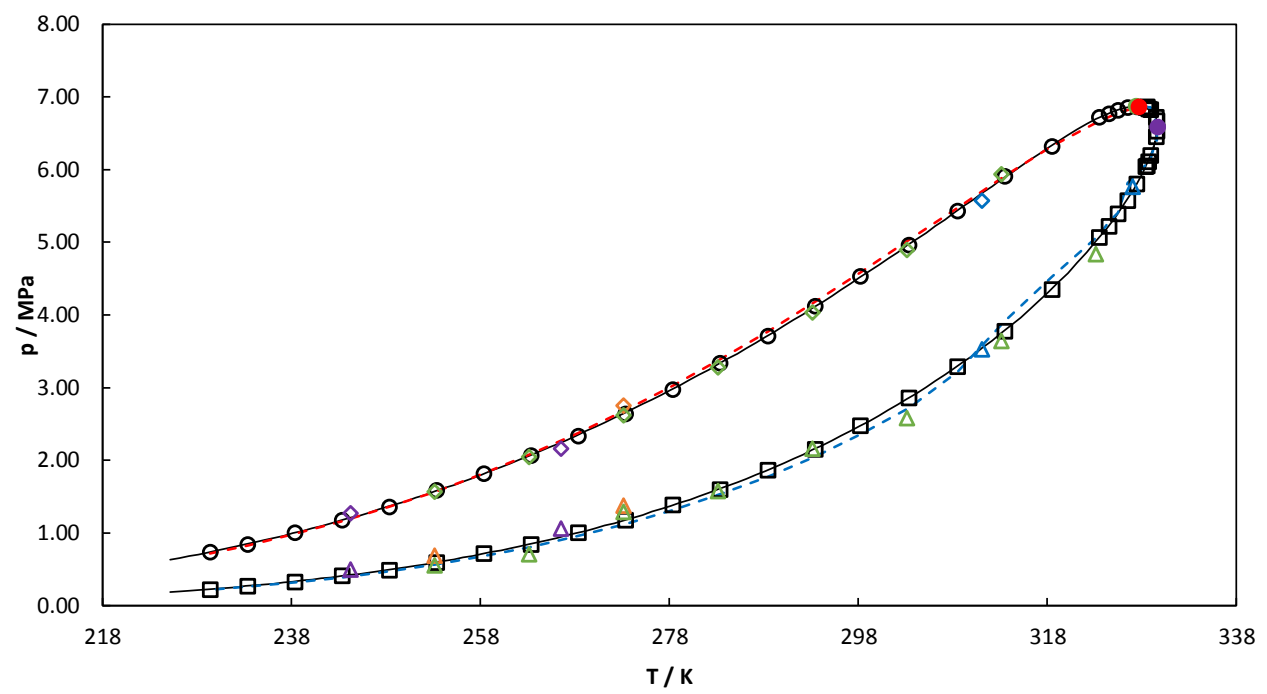

Figura 6.6Envolvente de fases para el sistema $\left(\mathrm{x}_{1}\right) \mathrm{CO}_{2}+\left(1-\mathrm{x}_{1}\right) \mathrm{C}_{3} \mathrm{H}_{8}\left(\mathrm{x}_{1}=0.60053\right)$; punto de burbuja (o) rocío ( $\left.\square\right)$ esta

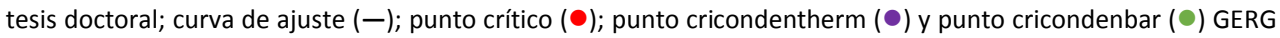
2008 [4]; curva de ajuste de Peng-Robinson [17] \& Wong-Sandler [18] burbuja (- -) y rocío (- -); Niesen et al. [85] burbuja $(\diamond)$, rocío $(\Delta)$; Akers et al. [86] burbuja $(\diamond)$, rocío $(\Delta)$; Hamam et al. [87] burbuja ( $\diamond)$, rocío $(\Delta)$; Kim et al. [72] burbuja $(\diamond)$, rocío $(\Delta)$.

De los datos encontrados en la literatura, Niesen et al. [85], Akers et al. [86] y Hamam et al. [87], ha sido Kim et al. [72] el que mayor número de datos del equilibrio líquido vapor a diferentes isotermas se ha podido comparar con el sistema presentado en esta tesis doctoral, estos datos experimentales se encuentran a composiciones muy cercanas a las estudiadas en esta tesis doctoral.

Niesen et al. [85] a composiciones de 0.579 y 0.592 para los puntos de burbuja y rocío respectivamente y Kim et al. [72] a composiciones de 0.582 y 0.615 (burbuja y rocío) para la isoterma de $273.15 \mathrm{~K}$, para la isoterma de $283.15 \mathrm{~K}$ la composición en el punto de burbuja fue de 0.590 ; a $293.15 \mathrm{~K}$ otros dos puntos de composición 0.584 y 0.590 (burbuja y rocío), en la isoterma de $303.15 \mathrm{~K}$ nos encontramos con un nuevo punto muy cercano a los estudiados en esta tesis con composición de 0.599 para el punto de burbuja, y un último punto a la isoterma de $313.15 \mathrm{~K}$ y de composición 0.584 para el punto de rocío. 
Las desviaciones con respecto a la ecuación GERG 2008 [4], de los datos experimentales de $\mathrm{Kim}$ et al. [72] donde presenta desviaciones porcentuales promedio en la medida del punto de burbuja de $0.8 \%$, mientras que las desviaciones porcentuales en las medidas del punto de rocío son del $4.2 \%$. Todos estos valores corresponden a la zona del punto de rocío mientras que el resto de valores las desviaciones que Kim et al. [72] presenta no superan el 4\%. La figura 6.7 muestras las desviaciones porcentuales de los datos experimentales estudiados en esta tesis doctoral y su comparación bibliográfica.

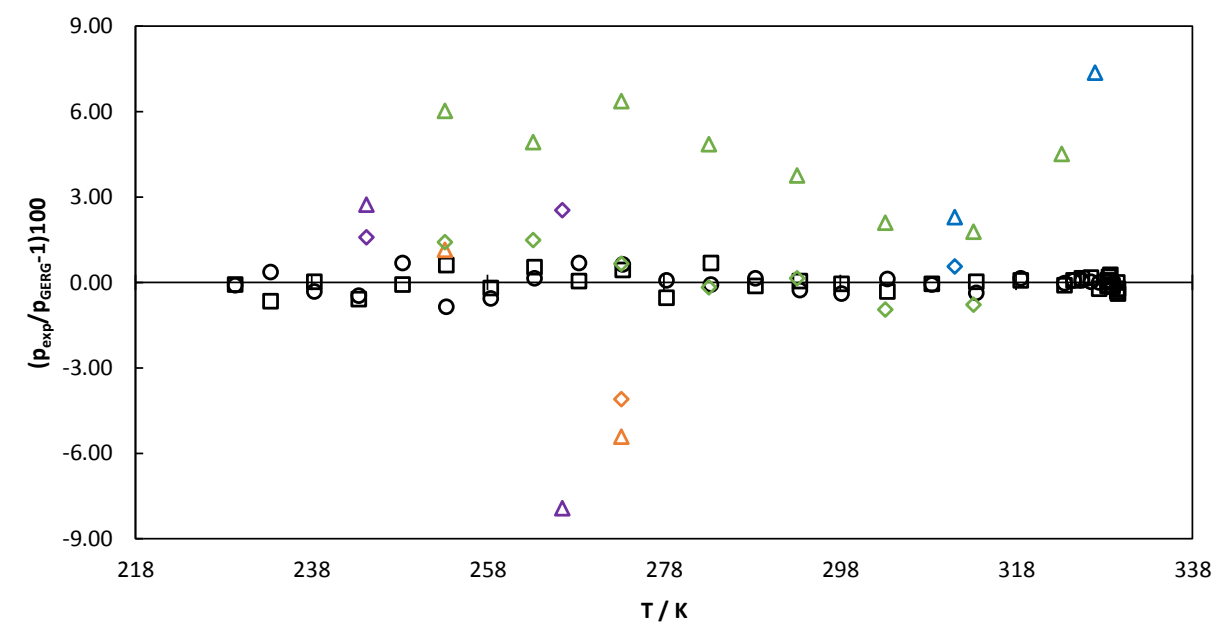

Figura 6.7Desviaciones relativas respecto la ecuación GERG-2008 [4] para el sistema $\left(\mathrm{x}_{1}\right) \mathrm{CO}_{2}+\left(1-\mathrm{x}_{1}\right) \mathrm{C}_{3} \mathrm{H}_{8}$ $\left(x_{1}=0.60053\right)$, punto de burbuja (o) rocío ( $\square$ ) esta tesis doctoral, Niesen et al. [85] burbuja $(\diamond)$, rocío $(\Delta)$; Akers et al. [86] burbuja ( $\diamond)$, rocío $(\Delta)$; Hamam et al. [87] burbuja ( $\diamond)$, rocío $(\Delta)$; Kim et al. [72] burbuja ( $\diamond)$, rocío $(\Delta)$.

Como puede observarse los datos presentados para este sistema mediante esta nueva celda de medida no superan los $2 \%$ mientras que Niesen et al. [85] alcanza un valor de desviaciones en la isoterma de $327.00 \mathrm{~K}$ del $7 \%$,al igual que Hamam et al. [87], cuya desviación el a isoterma de $266.48 \mathrm{~K}$ alcanza el $8 \%$ en la desviación de los datos. Por otro lado al igual que se mencionó anteriormente, es Kim et al. [72] 
con su gran cantidad de isotermas estudiadas las que más se asemejan a las desviaciones obtenidas. 


\subsubsection{Sistema binario $(0.8) \mathrm{CO}_{2}+(0.2) \mathrm{C}_{3} \mathrm{H}_{8}$}

La tabla 6.6 muestra los datos experimentales juntos con los calculados y sus deviaciones correspondientes del sistema dióxido de carbono + propano a una composición de 0.79777 en fracción molar de dióxido de carbono. A igual que el sistema visto en apartado anterior para este nuevo sistema, la envolvente de fases fue determinada entre el rango de temperaturas de (233.15 a 310.36$) \mathrm{K}$ y en el rango de presiones de (0.4 a 6.7) MPa. Debido a la proximidad entre los valores del punto de máxima temperatura (cricondentherm), que para esta composición se encuentra a $310.44 \mathrm{~K}$, y del punto crítico, cuyo valor en temperatura a la composición estudiada es de 310.45 K, no se han detectado zonas de condensación retrógrada.

\begin{tabular}{|c|c|c|c|c|c|}
\hline Ensayo & $T_{\exp } / \mathrm{K}$ & $\mathrm{p}_{\text {exp }} / \mathrm{MPa}$ & $f_{\text {exp }} / G H z$ & $\mathrm{p}_{\mathrm{GERG}} / \mathrm{MPa}$ & $\delta \mathrm{p} \%$ \\
\hline Burbuja & 233.36 & 0.923 & 4.1330 & 0.921 & -0.17 \\
\hline Burbuja & 238.37 & 1.095 & 4.1326 & 1.098 & 0.24 \\
\hline Burbuja & 243.36 & 1.296 & 4.1323 & 1.297 & 0.07 \\
\hline Burbuja & 248.35 & 1.522 & 4.1319 & 1.521 & -0.06 \\
\hline Burbuja & 253.36 & 1.775 & 4.1315 & 1.772 & -0.14 \\
\hline Burbuja & 258.35 & 2.056 & 4.1311 & 2.050 & -0.27 \\
\hline Burbuja & 263.35 & 2.362 & 4.1308 & 2.359 & -0.15 \\
\hline Burbuja & 268.34 & 2.707 & 4.1304 & 2.697 & -0.37 \\
\hline Burbuja & 273.32 & 3.072 & 4.1300 & 3.068 & -0.13 \\
\hline Burbuja & 278.34 & 3.478 & 4.1297 & 3.475 & -0.07 \\
\hline Burbuja & 283.33 & 3.911 & 4.1293 & 3.916 & 0.12 \\
\hline Burbuja & 288.33 & 4.409 & 4.1293 & 4.393 & -0.35 \\
\hline Burbuja & 293.34 & 4.921 & 4.1290 & 4.909 & -0.26 \\
\hline Burbuja & 298.33 & 5.471 & 4.1288 & 5.457 & -0.25 \\
\hline Burbuja & 303.34 & 6.038 & 4.1289 & 6.035 & -0.06 \\
\hline Burbuja & 308.34 & 6.614 & 4.1301 & 6.602 & -0.19 \\
\hline
\end{tabular}


Tabla 6.5 Continuación.

\begin{tabular}{|c|c|c|c|c|c|}
\hline Ensayo & $\mathrm{T}_{\exp } / \mathrm{K}$ & $\mathrm{p}_{\mathrm{exp}} / \mathrm{MPa}$ & $f_{\text {exp }} / G H z$ & $\mathrm{p}_{\mathrm{geRG}} / \mathrm{Mpa}$ & $\delta \mathbf{p} \%$ \\
\hline Burbuja & 308.54 & 6.632 & 4.1302 & 6.622 & -0.15 \\
\hline Burbuja & 308.95 & 6.684 & 4.1303 & 6.661 & -0.33 \\
\hline Burbuja & 309.16 & 6.695 & 4.1304 & 6.681 & -0.21 \\
\hline Burbuja & 309.35 & 6.714 & 4.1305 & 6.697 & -0.25 \\
\hline Burbuja & 309.55 & 6.726 & 4.1306 & 6.714 & -0.19 \\
\hline Burbuja & 309.96 & 6.746 & 4.1307 & 6.741 & -0.08 \\
\hline Burbuja & 310.16 & 6.747 & 4.1308 & 6.750 & 0.05 \\
\hline Burbuja & 310.36 & 6.772 & 4.1309 & 6.758 & -0.21 \\
\hline Rocío & 310.36 & 6.510 & 4.1318 & 6.517 & 0.11 \\
\hline Rocío & 310.16 & 6.451 & 4.1318 & 6.462 & 0.17 \\
\hline Rocío & 309.96 & 6.420 & 4.1318 & 6.415 & -0.07 \\
\hline Rocío & 309.55 & 6.328 & 4.1319 & 6.322 & -0.09 \\
\hline Rocío & 309.35 & 6.285 & 4.1319 & 6.277 & -0.12 \\
\hline Rocío & 309.16 & 6.244 & 4.1320 & 6.237 & -0.12 \\
\hline Rocío & 308.95 & 6.195 & 4.1320 & 6.194 & -0.03 \\
\hline Rocío & 308.54 & 6.117 & 4.1321 & 6.113 & -0.06 \\
\hline Rocío & 308.34 & 6.073 & 4.1322 & 6.075 & 0.04 \\
\hline Rocío & 303.34 & 5.265 & 4.1329 & 5.241 & -0.46 \\
\hline Rocío & 298.33 & 4.544 & 4.1340 & 4.547 & 0.06 \\
\hline Rocío & 293.34 & 3.916 & 4.1347 & 3.945 & 0.75 \\
\hline Rocío & 288.33 & 3.411 & 4.1354 & 3.412 & 0.04 \\
\hline Rocío & 283.33 & 2.954 & 4.1362 & 2.942 & -0.40 \\
\hline Rocío & 278.34 & 2.526 & 4.1368 & 2.527 & 0.08 \\
\hline Rocío & 273.32 & 2.162 & 4.1375 & 2.159 & -0.11 \\
\hline Rocío & 268.34 & 1.839 & 4.1382 & 1.838 & -0.06 \\
\hline Rocío & 263.35 & 1.545 & 4.1389 & 1.556 & 0.68 \\
\hline Rocío & 258.35 & 1.305 & 4.1395 & 1.308 & 0.25 \\
\hline Rocío & 253.36 & 1.090 & 4.1400 & 1.094 & 0.34 \\
\hline Rocío & 248.35 & 0.903 & 4.1408 & 0.908 & 0.52 \\
\hline Rocío & 243.36 & 0.748 & 4.1414 & 0.748 & 0.00 \\
\hline Rocío & 238.37 & 0.609 & 4.1420 & 0.612 & 0.49 \\
\hline Rocío & 233.36 & 0.497 & 4.1426 & 0.495 & -0.31 \\
\hline
\end{tabular}


De igual manera, no se ha observado la presencia de estados metaestables en el transcurso de las medidas, se han calculado las desviaciones porcentuales tomando como referencia los valores devueltos mediante la ecuación GERG 2008 [4], con un promedio de una desviación porcentual de $0.18 \%$ para las medidas del punto de burbuja, mientras que para las medidas del punto de rocío se han obtenido unas desviaciones promedio de $0.22 \%$. la incertidumbre expandida combinada para un factor de cobertura de $\mathrm{k}=2$ en la mediad de la temperatura fue de $U(T)=0.05 \mathrm{~K}$ y en la medida de la presión de $\mathrm{U}(\mathrm{p})=0.010 \mathrm{MPa}$, mientras que la incertidumbre relativa en el rango de (0.497 a 6.772) MPa en la envolvente fue de $U_{r}(p)=2.01 \%(\mathrm{MPa} / \mathrm{MPa})$ y $\mathrm{U}_{r}(\mathrm{p})=0.15 \%(\mathrm{MPa} / \mathrm{MPa})$ respectivamente

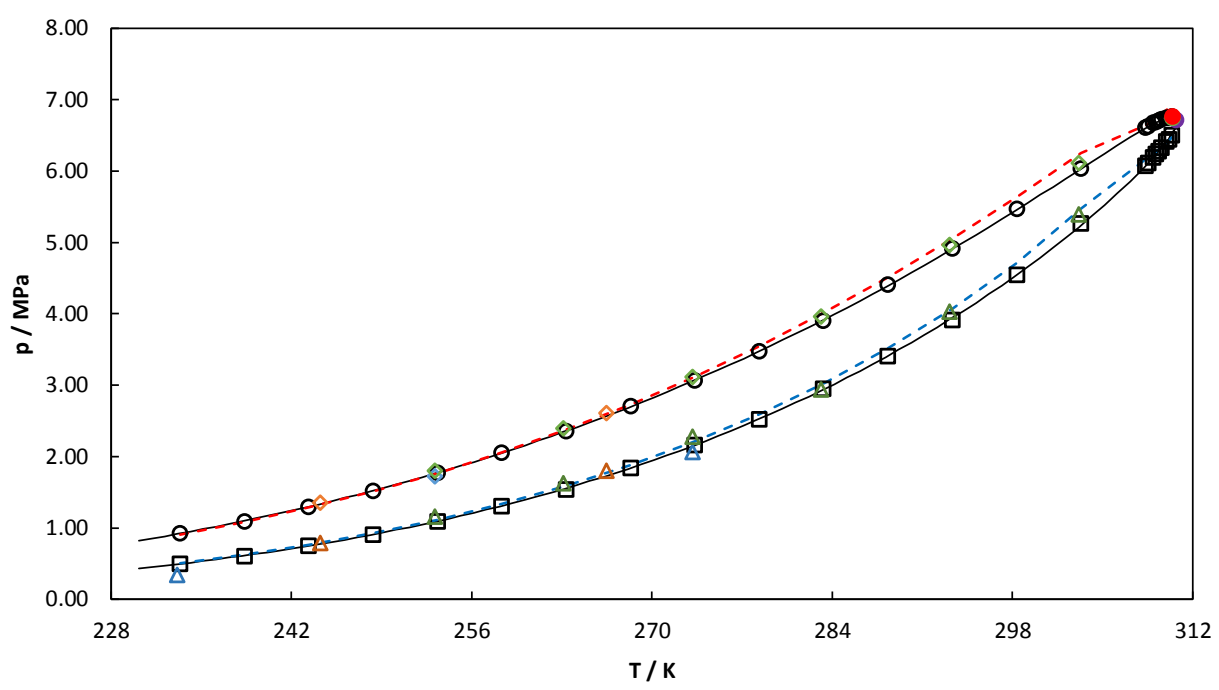

Figura 6.8Envolvente de fases para el sistema $\left(\mathrm{x}_{1}\right) \mathrm{CO}_{2}+\left(1-\mathrm{x}_{1}\right) \mathrm{C}_{3} \mathrm{H}_{8}\left(\mathrm{x}_{1}=0.79777\right)$; punto de burbuja (o) rocío ( $\square$ ) esta tesis doctoral; curva de ajuste $(-)$; punto crítico $(\bullet)$; punto cricondentherm $(\bullet)$; y punto cricondenbar $(\bullet)$ GERG 2008 [4]; curva de ajuste de Peng-Robinson [17] \& Wong-Sandler [18] burbuja (- -) y rocío (--); Akers et al. [86] burbuja $(\diamond)$, rocío $(\Delta)$; Hamam et al. [87] burbuja ( () , rocío $(\Delta)$; Kim et al. [72] burbuja ( $)$, rocío $(\Delta)$.

La figura 6.8 muestra la figura de la envolvente de fases de sistema estudiado, también se muestra la curva calculada mediante la ecuación GERG 2008 [4] además de la modelización de estos datos experimentales por medio de la ecuación cúbica de estado de Peng-Robinson [17] mediante las reglas de mezcla 
de Wong-Sandler [18] obteniendo los parámetros de ajuste de cada uno de los sistemas los cuales están descritos al final de este capítulo. Para la comparación con la bibliografía se han utilizado las referencias: Akers et al. [86],. Hamam et al. [87], Kim et al. [72].

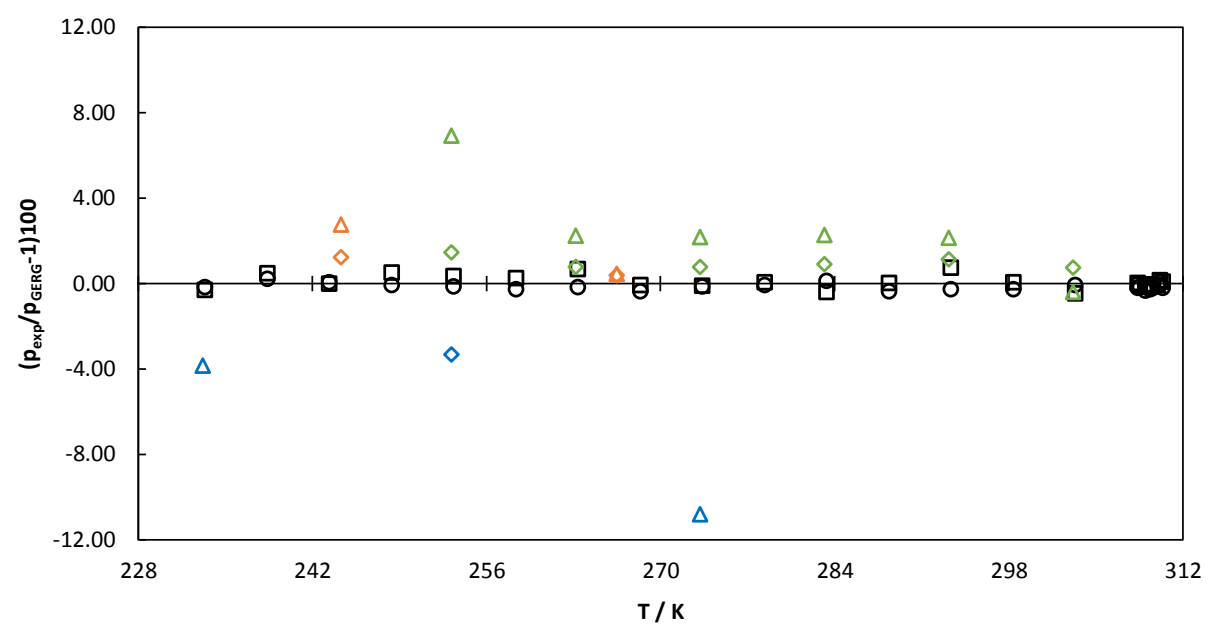

Figura 6.9Desviaciones relativas respecto la ecuación GERG-2008 [4] para el sistema $\left(\mathrm{x}_{1}\right) \mathrm{CO}_{2}+\left(1-\mathrm{x}_{1}\right) \mathrm{C}_{3} \mathrm{H}_{8}$ $\left(x_{1}=0.79777\right)$; punto de burbuja (o) rocío ( $\square$ ) esta tesis doctoral; Akers et al. [86] burbuja $(\diamond)$, rocío $(\Delta)$; Hamam et al. [87] burbuja $(\diamond)$, rocío $(\Delta)$; Kim et al. [72] burbuja $(\diamond)$, rocío $(\Delta)$.

Al igual en el sistema anteriormente descrito los sistemas estudiados por Kim et al. [72] son los que tienen composiciones más próximas a la estudiada en esta tesis doctoral y presenta valores de desviaciones porcentuales más bajas tal y como se muestra en la figura 6.9.

Comenzando por la isoterma $303.15 \mathrm{~K}$ dos puntos burbuja y rocío de composiciones 0.087 y 0.812 presentan devuelven desviaciones porcentuales del $0.74 \%$ para el punto de burbuja y $-0.40 \%$ para el punto de rocío. A la temperatura de $293.15 \mathrm{~K}$ el punto de burbuja con una composición de 0.803 tiene una desviación porcentual del $1.15 \%$ mientras que el punto de rocío de composición 0.800 nos devuelve una desviación del 2.13\%. A 283.15 K, Kim et al. [72] dos nuevos 
valores de composición fueron encontrados el primero de 0.808 y una desviación del $0.90 \%$ para el punto de burbuja, y el segundo de composición 0.793 y composición $2.26 \%$ correspondiente al punto de rocío. Dos valores más de composición 0.814 y 0.808 con desviaciones de $0.78 \%$ y $2.24 \%$ para los puntos de burbuja y rocío respectivamente fueron hallados en la isoterma $273.15 \mathrm{~K}$. Un último punto en la isoterma $263.15 \mathrm{~K}$ a composiciones de 0.820 y 0.806 dieron desviaciones de $0.78 \%$ y $2.25 \%$ para los puntos de burbuja y rocío respectivamente. Para este sistema las desviaciones medias porcentuales de los datos experimentales obtenidos no superan el $0.7 \%$ como valor máximo.

Kim et al. [72] en la isoterma $253.15 \mathrm{~K}$ da un valor en la desviación de porcentual de $6.92 \%$ para el punto de rocío en la composición de 0.798. Mientras que Akers et al. [86] a la isoterma de $273.15 \mathrm{~K}$ presenta uno de los valores más elevados en cuanto a desviaciones porcentuales se refiere con una valor del $10.79 \%$ y a una composición en el punto de rocío de 0.810 , siendo el valor promedio. Las desviaciones porcentuales promedio para los datos experimentales de Akers et al. [86] han sido de $3.32 \%$ para el punto de burbuja y de $7.13 \%$ para el punto de rocío, mientras que para los valores dados por Hamam et al. [87] las desviaciones porcentuales obtenidas han sido de $0.82 \%$ para los puntos de burbuja y de $1.6 \%$ para los punto de rocío. No se ha encontrado en la literatura medidas experimentales por encima de los $310.15 \mathrm{~K}$ correspondientes a la zona de máxima temperatura (cricondentherm) a la composición estudiada en esta tesis doctoral, así mismo el punto crítico se encuentra muy próximo al punto cricondentherm, 310.45 K y 310.71 K respectivamente de acuerdo con la ecuación GERG 2008 [4], esto origina que no se hayan podido determinar puntos de condensación retrógrada en este sistema binario. 


\subsubsection{Sistema binario $(0.5) \mathrm{CO}_{2}+(0.5) \mathrm{N}_{2}$}

En la tabla 6.7 se muestran los valores experimentales del sistema dióxido de carbono + nitrógeno a una composición de 0.49905 en fracción molar de dióxido de carbono. El sistema fue medido entre el rango de temperaturas de (229.37 a 262.63) K y en el rango de presiones de (1.9 a 18.8) MPa incluida la zona retrógrada considerando el punto crítico de referencia. La incertidumbre expandida combinada para un factor de cobertura de $k=2$ en la medida de la temperatura fue de $U(T)=0.05 \mathrm{~K}$ y en la medida de la presión de $U(p)=0.010 \mathrm{MPa}$, mientras que la incertidumbre relativa en el rango de (1.993 a 18.790) MPa en la envolvente fue de $U_{r}(p)=0.50 \%(\mathrm{MPa} / \mathrm{MPa})$ y $U_{r}(\mathrm{p})=0.05 \%(\mathrm{MPa} / \mathrm{MPa})$ respectivamente. No se ha observado la presencia de estados metaestables en el transcurso de las medidas, las desviaciones porcentuales se han calculado tomando como referencia los valores obtenidos mediante la ecuación GERG 2008 [4]. Se observa que la desviación media en la determinación del punto de burbuja ha sido del $0.008 \%$, mientras que dicha desviación para la determinación del punto de rocío ha sido del $0.15 \%$.

Tabla 6.7 Datos experimentales de los puntos de burbuja, rocío y frecuencia de resonancia del sistema $(0.49905) \mathrm{CO}_{2}+(0.50095) \mathrm{N}_{2}$ con una incertidumbre expandida $(\mathrm{k}=2)$ en $\mathrm{U}(\mathrm{T})=0.05 \mathrm{~K} ; \mathrm{U}(\mathrm{p})=0.010 \mathrm{MPa}$. Presiones calculadas mediante la ecuación de estado de GERG 2008 [4].

\begin{tabular}{l|ccccc} 
Ensayo & $\mathbf{T}_{\text {exp }} / \mathbf{K}$ & $\mathbf{p}_{\text {exp }} / \mathbf{M P a}$ & $\mathbf{f}_{\text {exp }} / \mathbf{G H z}$ & $\mathbf{p}_{\text {GERG }} / \mathbf{M P a}$ & $\delta \mathbf{p} \%$ \\
\hline Burbuja & 229.37 & 18.790 & 4.1337 & 18.788 & -0.01 \\
Burbuja & 233.35 & 18.177 & 4.1341 & 18.176 & -0.01 \\
Rocío* & 238.36 & 17.431 & 4.1343 & 17.433 & 0.01 \\
Rocío* & 241.35 & 16.972 & 4.1345 & 16.975 & 0.02 \\
Rocío* & 245.35 & 16.308 & 4.1350 & 16.314 & 0.04 \\
Rocío* & 249.33 & 15.559 & 4.1353 & 15.568 & 0.06 \\
Rocío* & 253.35 & 14.693 & 4.1357 & 14.685 & -0.06 \\
Rocío* & 255.35 & 14.153 & 4.1359 & 14.171 & 0.13 \\
Rocío* & 258.34 & 13.222 & 4.1366 & 13.249 & 0.20 \\
Rocío* & 260.35 & 12.402 & 4.1368 & 12.429 & 0.22
\end{tabular}


Tabla 6.6 Continuación

\begin{tabular}{|c|c|c|c|c|c|}
\hline Ensayo & $\mathrm{T}_{\text {exp }} / \mathrm{K}$ & $\mathrm{p}_{\mathrm{exp}} / \mathrm{MPa}$ & $\mathbf{f}_{\exp } / \mathbf{G H z}$ & p & $\delta \mathbf{p} \%$ \\
\hline Rocío* & 262.34 & 11.026 & 4.1373 & 11.019 & -0.06 \\
\hline Rocío* & 262.45 & 10.881 & 4.1373 & 10.871 & -0.09 \\
\hline Rocío* & 262.54 & 10.712 & 4.1374 & 10.704 & -0.08 \\
\hline Rocío* & 262.63 & 10.458 & 4.1376 & 10.456 & -0.02 \\
\hline Rocío* & 262.63 & 9.811 & 4.1375 & 9.829 & 0.19 \\
\hline Rocío & 262.54 & 9.576 & 4.1377 & 9.577 & 0.01 \\
\hline Rocío & 262.45 & 9.403 & 4.1378 & 9.406 & 0.03 \\
\hline Rocío & 262.34 & 9.255 & 4.1379 & 9.254 & -0.01 \\
\hline Rocío & 260.35 & 7.824 & 4.1385 & 7.779 & -0.57 \\
\hline Rocío & 257.34 & 6.588 & 4.1389 & 6.561 & -0.42 \\
\hline Rocío & 255.34 & 5.976 & 4.1393 & 5.953 & -0.38 \\
\hline Rocío & 253.35 & 5.454 & 4.1397 & 5.435 & -0.35 \\
\hline Rocío & 249.32 & 4.581 & 4.1402 & 4.567 & -0.31 \\
\hline Rocío & 245.35 & 3.880 & 4.1408 & 3.869 & -0.29 \\
\hline Rocío & 241.34 & 3.290 & 4.1413 & 3.281 & -0.27 \\
\hline Rocío & 238.36 & 2.909 & 4.1417 & 2.902 & -0.26 \\
\hline Rocío & 235.35 & 2.562 & 4.1421 & 2.562 & 0.01 \\
\hline Rocío & 233.35 & 2.358 & 4.1423 & 2.358 & -0.01 \\
\hline Rocío & 229.37 & 1.993 & 4.1428 & 1.992 & -0.04 \\
\hline
\end{tabular}

Punto de rocío retrógrado considerando el punto crítico calculado mediante la GERG 2008 [4].

La figura 6.10 muestra la envolvente de fases obtenida experimentalmente también se ha representado el resultado de la modelización mediante la ecuación cúbica de estado de Peng-Robinson [17] empleando las reglas de mezcla de WongSandler [18] así como la curva de ajuste por medio de la ecuación GERG 2008 [4], además se han incluido en la figura los valores experimentales de diferentes autores que han medido el equilibrio líquido vapor de este sistema. 


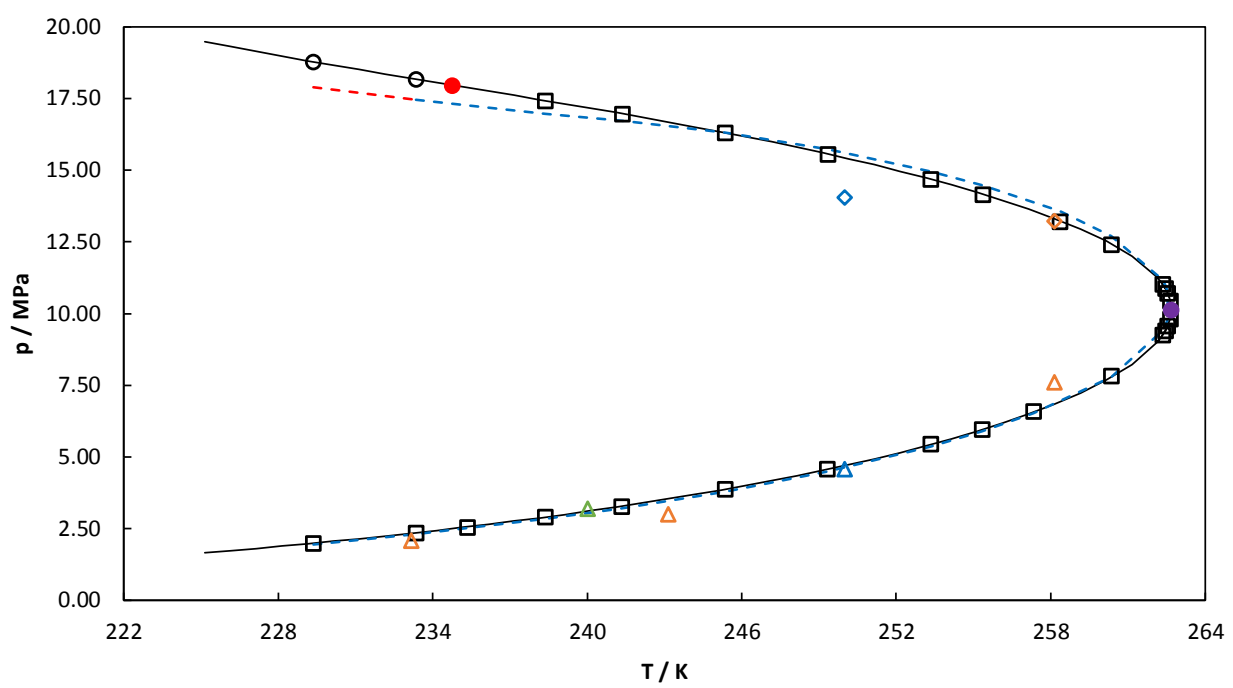

Figura 6.10 Envolvente de fases para el sistema $\left(x_{1}\right) C O_{2}+\left(1-x_{1}\right) N_{2}\left(x_{1}=0.49905\right)$; punto de burbuja (o) rocío ( $\left.\square\right)$ esta tesis doctoral; curva de ajuste (-); punto crítico $(\bullet)$; punto cricondentherm $(\bullet)$ y punto cricondenbar $(\bullet)$; GERG 2008 [4]; curva de ajuste de Peng-Robinson [17] \& Wong-Sandler [18] burbuja (- -) y rocío (--); Brown et al. [88] burbuja ( $\diamond)$, rocío $(\Delta)$; Fandiño et al. [89] burbuja ( $\diamond)$, rocío $(\Delta)$; Al Sahhaf et al. [83] rocío $(\Delta)$.

Brown et al. [88], Fandiño et al. [89] y Al Sahhaf et al. [83]. Pocos han sido los autores encontrados que hayan estudiado este sistema en el rango de isotermas a este sistema estudiado.

Se han determinado los puntos de condensación retrograda para este sistema pudiendo determinar cada uno de los valores a las isotermas seleccionadas, muchos han sido los puntos hallados en esta área dado que el punto crítico según GERG 2008 [4] se encuentra en 234.75 K y a 17.969 MPa de presión.

En la figura 6.11 se han representado las desviaciones porcentuales frente a la temperatura de los datos experimentales y la literatura encontrada cabe destacar que, aunque la literatura encontrada es escasa, las desviaciones porcentuales obtenidas son hasta ahora las más bajas. 


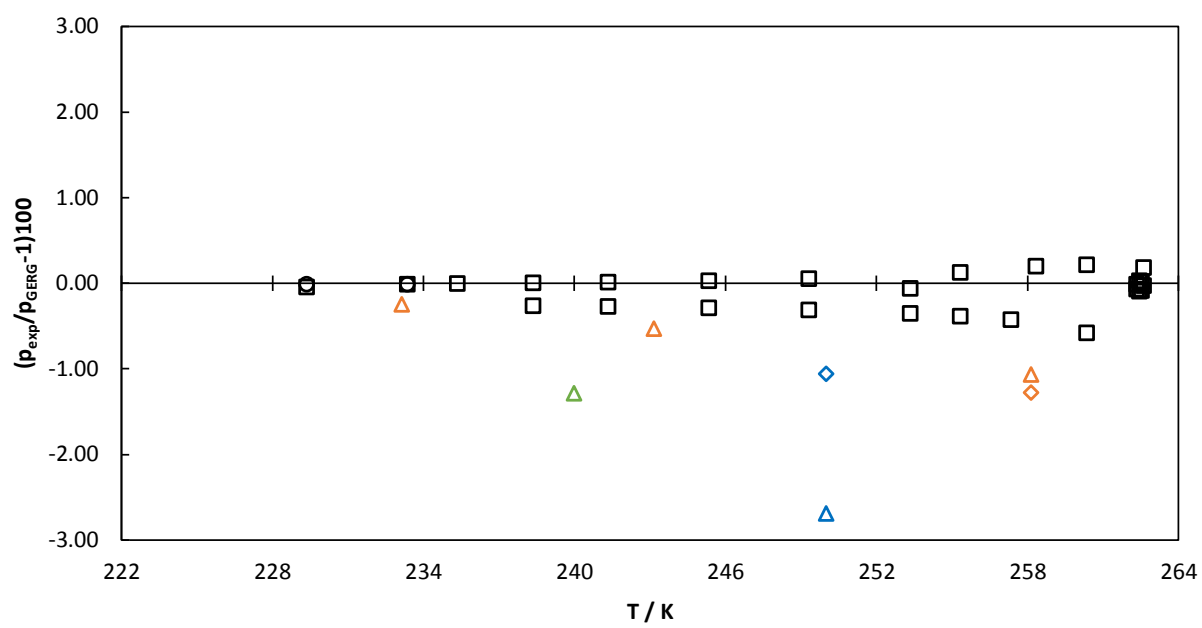

Figura 6.11 Desviaciones relativas respecto la ecuación GERG-2008 [4] para el sistema $\left(\mathrm{x}_{1}\right) \mathrm{CO}_{2}+\left(1-\mathrm{x}_{1}\right) \mathrm{N}_{2}$ $\left(x_{1}=0.49905\right)$; punto de burbuja (o) rocío $\left.(\square)\right)$ esta tesis doctoral; Brown et al. [88] burbuja ( $\left.\diamond\right)$, rocío $(\Delta)$; Fandiño et al. [89] burbuja ( $\diamond)$, rocío $(\triangle)$; Al Sahhaf et al. [83] rocío $(\Delta)$.

Las desviaciones de la GERG 2008 [4] de los datos con esta nueva celda de medida no superan el $0.6 \%$ mientras que Brown et al. [88], alcanza un valor de desviaciones en la isoterma de $250.00 \mathrm{~K}$ del $2.7 \%$, este es valor de desviación más elevado encontrado, el resto de autores reportan valores de desviaciones no superiores al 1.3\%. Fandiño et al. [89] obtiene muy buenos valores de desviaciones, para la isoterma $233.15 \mathrm{~K}$ la desviación obtenida es de $-0.24 \%$ para el punto de rocío y para la isoterma de $243.15 \mathrm{~K}$ la desviación obtenida es de $-0.53 \%$ también para el punto de rocío. Al Sahhaf et al. [83] a la isoterma de $240 \mathrm{~K}$ presenta un valor de desviación del -1.23\% para el punto de rocío. 


\subsubsection{Sistema binario $(0.8) \mathrm{CO}_{2}+(0.2) \mathrm{N}_{2}$}

La tabla 6.8 muestra el último de los sistemas presentado en esta tesis doctoral, en ella al igual que en las tablas anteriores, se muestran los valores experimentales obtenidos por medio la nueva celda de medida que se ha construido al igual que los valores de las frecuencias de resonancia asociadas a cada una de las isotermas. Dicha tabla muestra las medidas experimentales de la envolvente de fases del sistema dióxido de carbono + nitrógeno a la composición de 0.79777 en fracción molar de dióxido de carbono.

Tabla 6.8 Datos experimentales de los puntos de burbuja, rocío y frecuencia de resonancia del sistema $(0.79777) \mathrm{CO}_{2}+(0.20222) \mathrm{N}_{2}$ con una incertidumbre expandida $(k=2)$ en $U(T)=0.05 \mathrm{~K} ; \mathrm{U}(\mathrm{p})=0.010 \mathrm{MPa}$. Presiones calculadas mediante la ecuación de estado de GERG 2008 [4].

\begin{tabular}{l|ccccc} 
Ensayo & $\mathbf{T}_{\text {exp }} / \mathbf{K}$ & $\mathbf{p}_{\text {exp }} / \mathbf{M P a}$ & $\mathbf{f}_{\text {exp }} / \mathbf{G H z}$ & $\mathbf{p}_{\text {GERG }} / \mathbf{M P a}$ & $\delta \mathbf{p} \%$ \\
\hline Burbuja & 229.37 & 12.061 & 4.1349 & 12.080 & 0.16 \\
Burbuja & 233.35 & 11.853 & 4.1346 & 11.905 & 0.44 \\
Burbuja & 238.36 & 11.734 & 4.1343 & 11.734 & 0.00 \\
Burbuja & 248.33 & 11.450 & 4.1335 & 11.516 & 0.58 \\
Burbuja & 258.34 & 11.333 & 4.1329 & 11.400 & 0.59 \\
Burbuja & 268.32 & 11.227 & 4.1327 & 11.288 & 0.55 \\
Burbuja & 273.31 & 11.110 & 4.1324 & 11.175 & 0.59 \\
Burbuja & 278.33 & 10.901 & 4.1322 & 10.945 & 0.40 \\
Burbuja & 282.32 & 10.624 & 4.1323 & 10.608 & -0.15 \\
Burbuja & 283.31 & 10.512 & 4.1324 & 10.493 & -0.18 \\
Rocío* & 284.33 & 10.351 & 4.1325 & 10.353 & 0.02 \\
Rocío* & 285.34 & 10.246 & 4.1327 & 10.182 & -0.63 \\
Rocío* & 286.34 & 9.991 & 4.1331 & 9.968 & -0.22 \\
Rocío* & 287.33 & 9.696 & 4.1337 & 9.658 & -0.39 \\
Rocío & 287.33 & 8.330 & 4.1343 & 8.389 & 0.71 \\
Rocío & 286.34 & 7.978 & 4.1346 & 7.926 & -0.65 \\
Rocío & 285.34 & 7.595 & 4.1349 & 7.572 & -0.30 \\
Rocío & 284.33 & 7.224 & 4.1352 & 7.267 & 0.60 \\
Rocío & 283.31 & 6.979 & 4.1354 & 6.991 & 0.17 \\
Rocío & 282.32 & 6.765 & 4.1357 & 6.745 & -0.29 \\
& & & & &
\end{tabular}


Tabla 6.6 Continuación.

\begin{tabular}{l|rcccc} 
Ensayo & $\mathbf{T}_{\text {exp }} / \mathbf{K}$ & $\mathbf{p}_{\text {exp }} / \mathbf{M P a}$ & $\mathbf{f}_{\text {exp }} / \mathbf{G H z}$ & $\mathbf{p}_{\text {GERG }} / \mathbf{M p a}$ & $\delta \mathbf{p} \%$ \\
\hline Rocío & 278.33 & 5.870 & 4.1364 & 5.897 & 0.48 \\
Rocío & 273.31 & 4.997 & 4.1372 & 5.029 & 0.64 \\
Rocío & 268.32 & 4.283 & 4.1379 & 4.306 & 0.53 \\
Rocío & 258.34 & 3.145 & 4.1393 & 3.146 & 0.02 \\
Rocío & 248.33 & 2.255 & 4.1405 & 2.263 & 0.32 \\
Rocío & 238.36 & 1.586 & 4.1418 & 1.594 & 0.51 \\
Rocío & 233.35 & 1.323 & 4.1424 & 1.323 & -0.01 \\
Rocío & 229.37 & 1.126 & 4.1429 & 1.134 & 0.77 \\
Sp \% $=\left(\frac{\mathrm{p}_{\text {exp }}}{\mathrm{p}_{\text {GERG }}}-1\right) * 100$ & & & & \\
Punto de rocío retrógrado considerando el punto crítico calculado mediante la GERG 2008 [4].
\end{tabular}

Esta tabla muestra las presiones en los puntos de burbuja y rocío para cada una de las isotermas estudiadas además de la frecuencia de resonancia asociada a cada presión y temperatura en el rango de temperaturas de (229.36 a 287.33) K y en el rango de presiones de (1.1 a 12.1) MPa incluida la zona retrograda considerando el punto crítico de referencia. Las desviaciones medias porcentuales para el punto de burbuja obtenidas en el punto de burbuja, han sido $0.36 \%$, mientras que para el punto de rocío se han obtenido un valor de desviación media porcentual de $0.40 \%$. La incertidumbre expandida combinada para un factor de cobertura de $k=2$ en la medida de la temperatura fue de $\mathrm{U}(\mathrm{T})=0.05 \mathrm{~K}$ y en la medida de la presión de $U(p)=0.010 \mathrm{MPa}$, mientras que la incertidumbre relativa en el rango de (1.126 a 12.061) $\mathrm{MPa}$ en la envolvente fue de $U_{r}(p)=0.89 \%(\mathrm{MPa} / \mathrm{MPa})$ y $U_{r}(p)=0.08 \%$ (MPa/MPa) respectivamente.

La figura 6.12 muestra la envolvente de fases obtenida experimentalmente además se ha representado el resultado de la modelización mediante la ecuación cúbica de estado de Peng-Robinson [17] empleando las reglas de mezcla de WongSandler [18] así como la curva correspondiente a la ecuación GERG 2008 [4], además se han incluido en la figura los valores experimentales de diferentes 
autores que han medido el equilibrio liquido vapor de este sistema, a saber Brown et al. [88], Westman et al. [90], Fandiño et al. [89] y Al Sahhaf et al. [83].

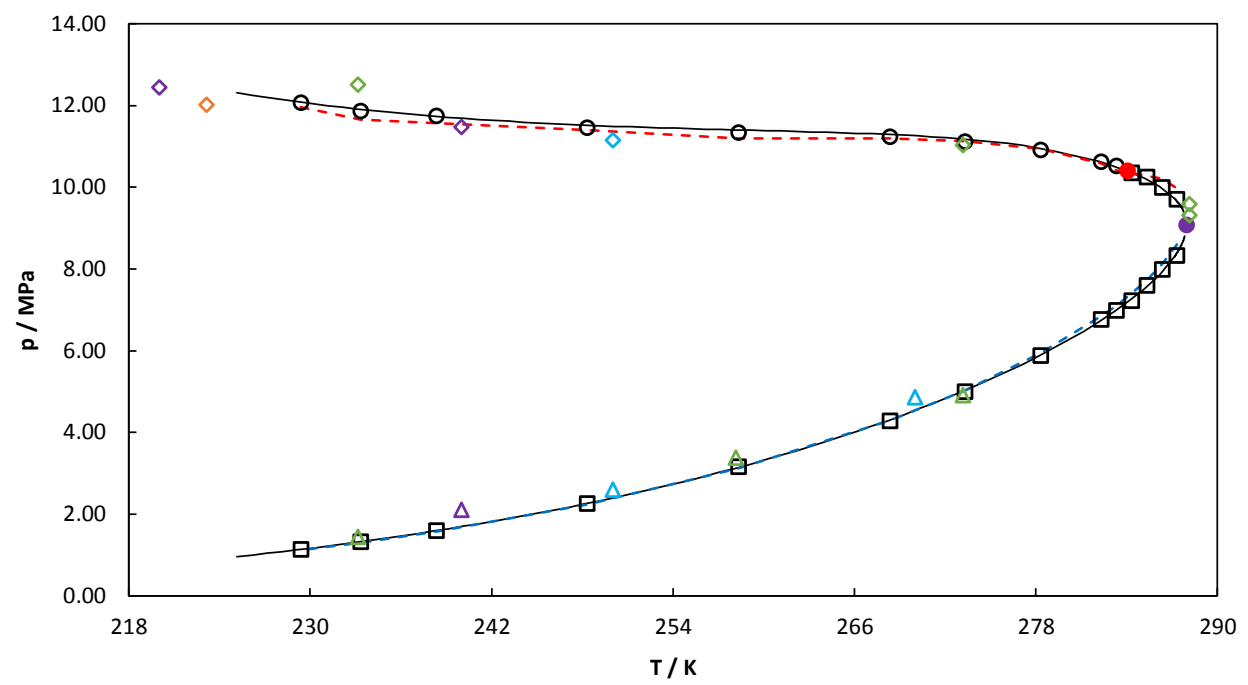

Figura 6.12 Envolvente de fases para el sistema $\left(x_{1}\right) \mathrm{CO}_{2}+\left(1-\mathrm{x}_{1}\right) \mathrm{N}_{2}\left(\mathrm{x}_{1}=0.79777\right)$; punto de burbuja (o) rocío ( $\left.\square\right)$

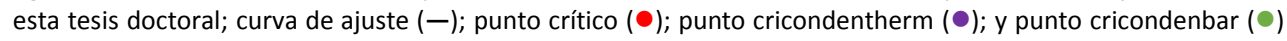
GERG 2008 [4]; curva de ajuste de Peng-Robinson [17] \& Wong-Sandler [18] burbuja ( - ) y rocío (--); Brown et al. [88] burbuja $(\diamond)$, rocío $(\Delta)$; Westman et al. [90] burbuja ( $\diamond)$; Fandiño et al. [89] burbuja ( $\diamond)$, rocío ( $\triangle$ ); Al Sahhaf et al. [83] burbuja $(\diamond)$, rocío $(\Delta)$.

En la figura 6.13, se muestran los resultados de las desviaciones porcentuales de los datos experimentales estudiados en esta tesis doctoral además de las desviaciones de los datos de la literatura, dichas desviaciones han sido comparadas en todos los casos con la ecuación de estado GERG 2008 [4]. Al igual que ene sistema anterior las desviaciones porcentuales de los datos experimentales no superan el $0.8 \%$. La literatura encontrada presenta valores porcentuales semejantes siendo los datos que más se aproximan a la composición bajo estudio los ofrecidos por Fandiño et al. [89] y Al Sahhaf et al. [83], donde las desviaciones no superan el $2 \%$. 


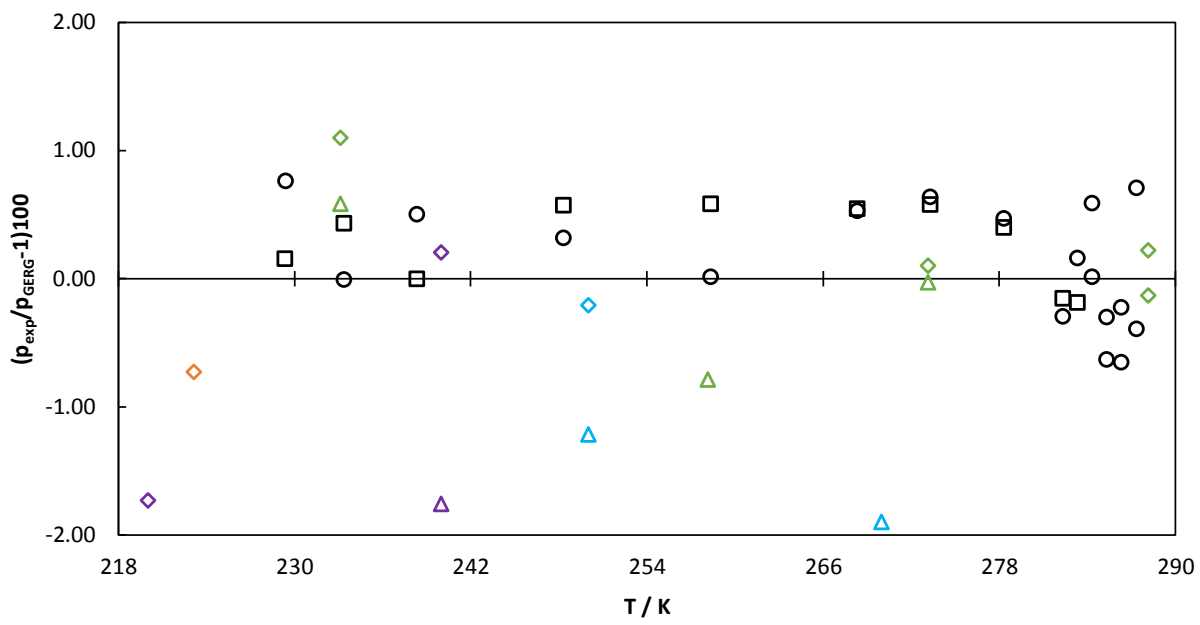

Figura 6.13 Desviaciones relativas respecto la ecuación GERG-2008 [4] para el sistema para el sistema $\left(\mathrm{x}_{1}\right) \mathrm{CO}_{2}+\left(1-\mathrm{x}_{1}\right) \mathrm{N}_{2}\left(\mathrm{x}_{1}=0.79777\right)$; punto de burbuja (o) rocío ( $\square$ ) esta tesis doctoral; Brown et al. [88] burbuja ( 0$)$, rocío $(\Delta)$; Westman et al. [90] burbuja $(\diamond)$; Fandiño et al. [89] burbuja ( $\diamond)$, rocío $(\Delta)$; Al Sahhaf et al. [83] burbuja $(\diamond)$, rocío $(\Delta)$.

Elegidos los autores que han determinado algún punto en común con la composición estudiada para este sistema, Brown et al. [88] que han obtenido para una composición de 0.807 una desviación en el punto de burbuja de $0.2 \%$, Westman et al. [90] a una composición de 0.805 en el punto de burbuja han obtenido una desviación del $-0.72 \%$. Fandiño et al. [89] son las que menores desviaciones porcentuales han obtenido en sus datos experimentales, comenzando con la isoterma $233.15 \mathrm{~K}$ y a una composición del punto de rocío de 0.258 , la desviación media porcentual obtenida es de $0.59 \%$, para la isoterma $258.15 \mathrm{~K}$ y a la composición de 0.753 la desviaciones en el punto de rocío es de $0.79 \%$.

Fandiño et al. [89] con otros dos puntos en la isoterma de $273.15 \mathrm{~K}$ a composiciones de 0.806 y 0.807 en los puntos de burbuja y rocío respectivamente ha obtenido desviaciones de $0.11 \%$ y $-0.02 \%$, otros dos datos esta vez ambos en la zona de burbuja en la isoterma de $288.15 \mathrm{~K}$ y a composiciones de 0.866 y 0.885 ha devuelto desviaciones de $-0.13 \%$ y $0.23 \%$ respectivamente. 


\subsection{Análisis de los resultados}

Seis sistemas que contiene dióxido de carbono, a diferentes composiciones en esta sustancia, han sido objeto de estudio en esta tesis doctoral, determinando su envolvente de fases en un amplio rango de presiones $y$ temperaturas $y$ comparando los resultados obtenidos por medio de la ecuación de estado GERG 2008 [4]. Además se ajustó el comportamiento de estas mezclas mediante la ecuación de estado cúbica de Peng-Robinson [17] empleando las reglas de mezcla de Wong-Sandler [18], obteniéndose los correspondientes parámetros. Todos los sistemas estudiados a excepción del sistema $(0.8) \mathrm{CO}_{2}+(0.2) \mathrm{C}_{3} \mathrm{H}_{8}$ presentan puntos de condensación retrógrada que han podido ser determinados mediante la técnica experimental presentada en esta tesis doctoral.

La tabla 6.9 muestra un resumen de las incertidumbres de medida, en primer lugar, se observa que independientemente del sistema que se trate, la incertidumbre debida a la temperatura no varía, esto es debido en primer lugar a la gran estabilidad de las sondas empleadas y en segundo lugar al meticuloso procedimiento de calibración que se emplea para determinar la incertidumbre de estas.

Tabla 6.9 Tabla resumen de las incertidumbres expandidas $(k=2)$ de los sistemas estudiados en esta tesis doctoral.

\begin{tabular}{|c|c|c|c|c|c|c|}
\hline Incertidumbre & \multicolumn{2}{|c|}{$\left(x_{1}\right) \mathrm{CO}_{2}+\left(1-x_{1}\right) \mathrm{CH}_{4}$} & \multicolumn{2}{|c|}{$\left(x_{1}\right) \mathrm{CO}_{2}+\left(1-x_{1}\right) \mathrm{C}_{3} \mathrm{H}_{8}$} & \multicolumn{2}{|c|}{$\left(\mathrm{x}_{1}\right) \mathrm{CO}_{2}+\left(1-\mathrm{x}_{1}\right) \mathrm{N}_{2}$} \\
\hline$\left(\mathrm{x}_{1}\right) \mathrm{CO}_{2}$ & 0.40064 & 0.60162 & 0.60053 & 0.79777 & 0.49905 & 0.79777 \\
\hline$U(T) / K$ & 0.05 & 0.05 & 0.05 & 0.05 & 0.05 & 0.05 \\
\hline $\mathrm{U}(\mathrm{p}) / \mathrm{MPa}$ & 0.010 & 0.010 & 0.010 & 0.010 & 0.010 & 0.010 \\
\hline
\end{tabular}


Con respecto a las incertidumbres debidas a la presión se aprecia los valores obtenidos no difieren mucho entre sí. Debido al procedimiento empleado en la determinación de los puntos experimentales la mayor contribución a la incertidumbre en la medida de la presión es la debida al procedimiento de linealizado en la determinación del punto experimental. La tabla 6.10 muestra un ejemplo del cálculo realizado para la determinación de la incertidumbre del punto de burbuja para el sistema dióxido de carbono + metano a una composición de 0.40064 en fracción molar de dióxido de carbono, para la isoterma T = 233.35 K.

Tabla 6.10 Tabla de incertidumbre para la determinación del punto de burbuja del sistema $(0.40064) \mathrm{CO}_{2}+(0.59936) \mathrm{CH}_{4}$ con una incertidumbre expandida $(\mathrm{k}=2)$ en la isoterma $\mathrm{T}=233.35 \mathrm{~K}$.

\begin{tabular}{c|lcccc} 
& Fuente & Unidad & Estimado & Divisor & $\mathbf{u}(\mathbf{x}) / \mathbf{M P a}$ \\
\hline \multirow{3}{*}{$\mathbf{u}(\mathbf{p})$} & Resolución & & 0.0001 & 1 & 0.0001 \\
& Repetibilidad & & 0.001 & 1 & 0.001 \\
& Calibración & $\mathrm{MPa}$ & 0.002 & 1 & 0.002 \\
& Polinomio & & 0.004 & 1 & 0.004 \\
& Composición & & $3 \cdot 10^{-6}$ & & $3 \cdot 10^{-6}$ \\
\hline \multirow{3}{*}{$\mathbf{u ( T )}$} & Resolución & & 0.001 & 1 & 0.001 \\
& Repetibilidad & $\mathrm{MPa}$ & $7 \cdot 10^{-5}$ & 1 & $7 \cdot 10^{-5}$ \\
& Calibración & & 0.03 & 1 & 0.03 \\
\hline $\mathbf{U}(\mathbf{p})$ & & $\mathrm{MPa}$ & & $\mathrm{k}=2$ & 0.010 \\
$\mathbf{U ( T )}$ & & $\mathrm{K}$ & & $\mathrm{k}=2$ & 0.05
\end{tabular}

La tabla 6.11, muestra los resultados de la modelización por medio de la ecuación cúbica de estado de Peng-Robinson [17] empleando las reglas de mezcla de Wong-Sandler [18]. Se ha escogido como parámetro no ajustable el que define la composición local (NRTL) [77] donde se recomienda que los valores en sistemas no simétricos esté comprendido entre 0.20 y 0.55 . De igual manera considerando la no asociación entre las mezclas empleadas en esta tesis doctoral se ha optado por el valor de 0.30 para este parámetro para modelar los sistemas con metano y 
nitrógeno, el sistema que contiene propano se ha ajustado mejor a un valor de 0.47 presumiblemente por la elevada asimetría entre las dos moléculas.

Tabla 6.11 Resultado de la predicción de la ecuación de estados de Peng \& Robinson [17] con reglas de mezcla de Wong \& Sandler [18].

\begin{tabular}{|c|c|c|c|c|c|c|}
\hline \multirow{2}{*}{$\frac{\text { Parámetros }}{\left(\mathrm{x}_{1}\right) \mathrm{CO}_{2}}$} & \multicolumn{2}{|c|}{$\left(x_{1}\right) \mathrm{CO}_{2}+\left(1-x_{1}\right) \mathrm{CH}_{4}$} & \multicolumn{2}{|c|}{$\left(x_{1}\right) \mathrm{CO}_{2}+\left(1-x_{1}\right) C_{3} H_{8}$} & \multicolumn{2}{|c|}{$\left(x_{1}\right) \mathrm{CO}_{2}+\left(1-x_{1}\right) \mathrm{N}_{2}$} \\
\hline & 0.40060 & 0.60162 & 0.60530 & 0.79777 & 0.49905 & 0.79777 \\
\hline $\mathbf{k}_{\mathrm{ij}}$ & 0.2211 & 0.2012 & 0.2778 & 0.3989 & 0.2438 & 0.2504 \\
\hline $\operatorname{NRTL}(\alpha)$ & 0.30 & 0.30 & 0.47 & 0.47 & 0.30 & 0.30 \\
\hline$\tau_{i j}$ & 1.5937 & 0.7473 & 4.1674 & 0.2598 & 0.7698 & 0.1607 \\
\hline$\tau_{\mathbf{j i}}$ & -0.0490 & 0.3911 & -0.2341 & 1.1233 & 0.1596 & 2.7311 \\
\hline$\sigma(p) / M P a$ & 0.1 & 0.1 & 0.1 & 0.1 & 0.3 & 0.1 \\
\hline
\end{tabular}

Se observa que las desviaciones medias en la presión son significativamente bajas ofreciendo un buen ajuste con los datos experimentales, incluso en las inmediaciones del punto crítico. Las figuras 6.14 a 6.19 muestran las desviaciones porcentuales de los sistemas estudiados comparado con la ecuación de estado respecto Peng-Robinson [17] empleando las reglas de mezcla de Wong-Sandler [18]. Como cabe esperar, aunque las desviaciones respecto a la presión de manera general son buenas, los datos experimentales observados individualmente ofrecen cierta desviación, sobre todo si comparamos este ajuste con el que ofrece la ecuación GERG 2008 [4], esto es debido a la complejidad propia de la ecuación de estado, aunque ambas estén basadas en las energías de Helmholtz, los parámetros termodinámicos de ajuste que emplea la ecuación GERG 2008 [4] son mucho mayores que los que emplea la ecuación de estado de Peng-Robinson [17] empleando las reglas de mezcla de Wong-Sandler [18]. De manera general las desviaciones porcentuales de los sistemas estudiados empleando esta ecuación de estado no son mayores del $6 \%$. 


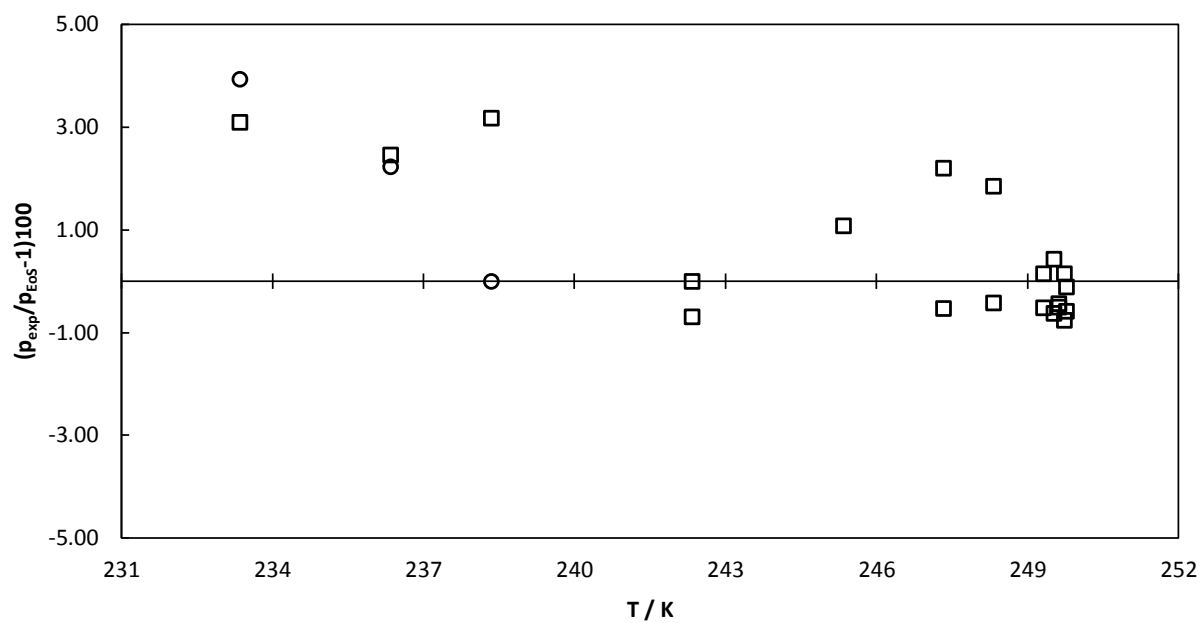

Figura 6.14 Desviaciones relativas respecto Peng-Robinson [17] empleando las reglas de mezcla de WongSandler [18] para el sistema $\left(\mathrm{x}_{1}\right) \mathrm{CO}_{2}+\left(1-\mathrm{x}_{1}\right) \mathrm{CH}_{4}\left(\mathrm{x}_{1}=0.40060\right)$, punto de burbuja (o) rocío ( $\square$ ) esta tesis doctoral.

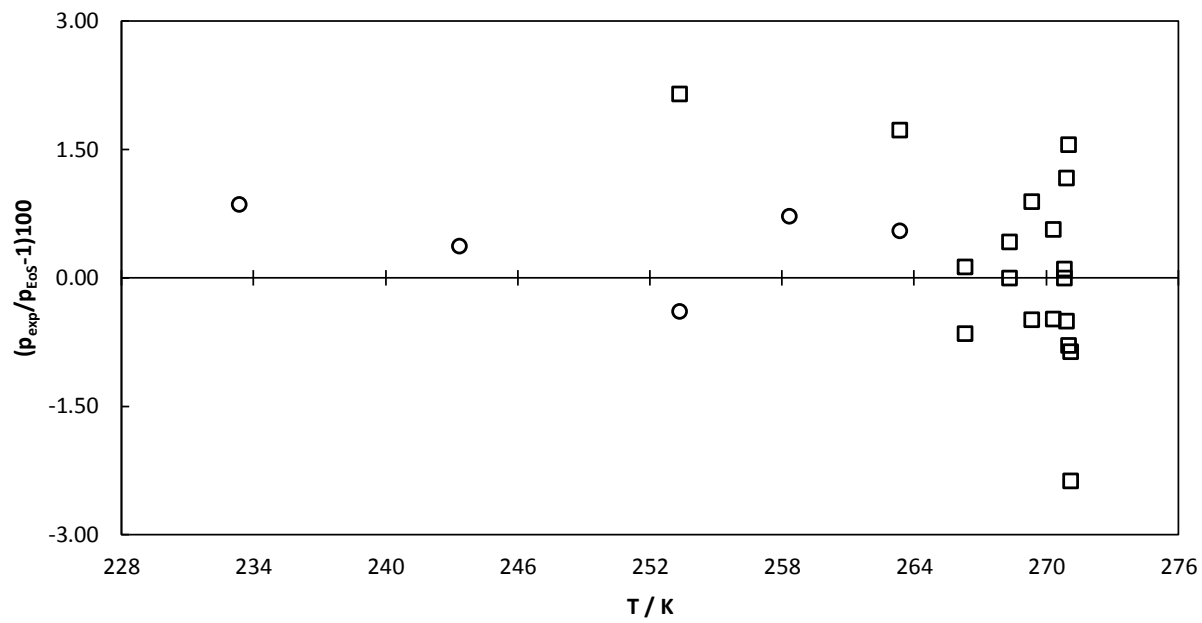

Figura 6.15 Desviaciones relativas respecto Peng-Robinson [17] empleando las reglas de mezcla de WongSandler [18] para el sistema $\left(\mathrm{x}_{1}\right) \mathrm{CO}_{2}+\left(1-\mathrm{x}_{1}\right) \mathrm{CH}_{4}\left(\mathrm{x}_{1}=0.60162\right)$, punto de burbuja (o) rocío ( $\square$ ) esta tesis doctoral. 


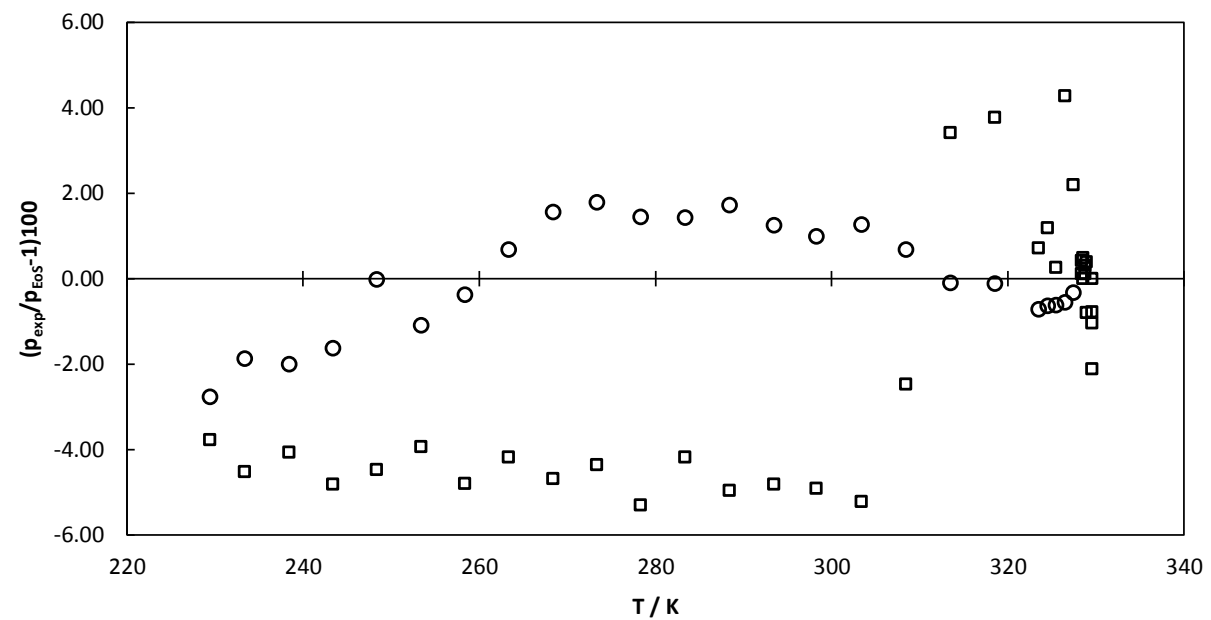

Figura 6.16 Desviaciones relativas respecto Peng-Robinson [17] empleando las reglas de mezcla de WongSandler [18] para el sistema $\left(\mathrm{x}_{1}\right) \mathrm{CO}_{2}+\left(1-\mathrm{x}_{1}\right) \mathrm{C}_{3} \mathrm{H}_{8}\left(\mathrm{x}_{1}=0.60053\right)$, punto de burbuja (o) rocío ( $\square$ ) esta tesis doctoral.

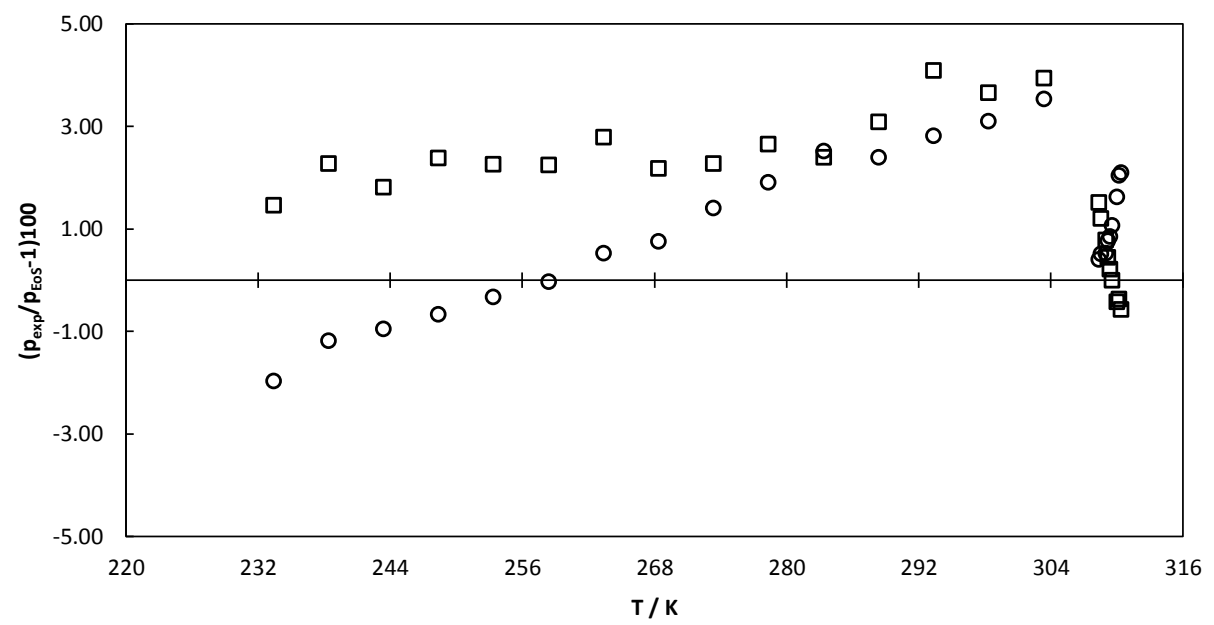

Figura 6.17 Desviaciones relativas respecto Peng-Robinson [17] empleando las reglas de mezcla de WongSandler [18] para el sistema $\left(\mathrm{x}_{1}\right) \mathrm{CO}_{2}+\left(1-\mathrm{x}_{1}\right) \mathrm{C}_{3} \mathrm{H}_{8}\left(\mathrm{x}_{1}=0.79777\right)$, punto de burbuja (o) rocío ( $\left.\square\right)$ esta tesis doctoral. 


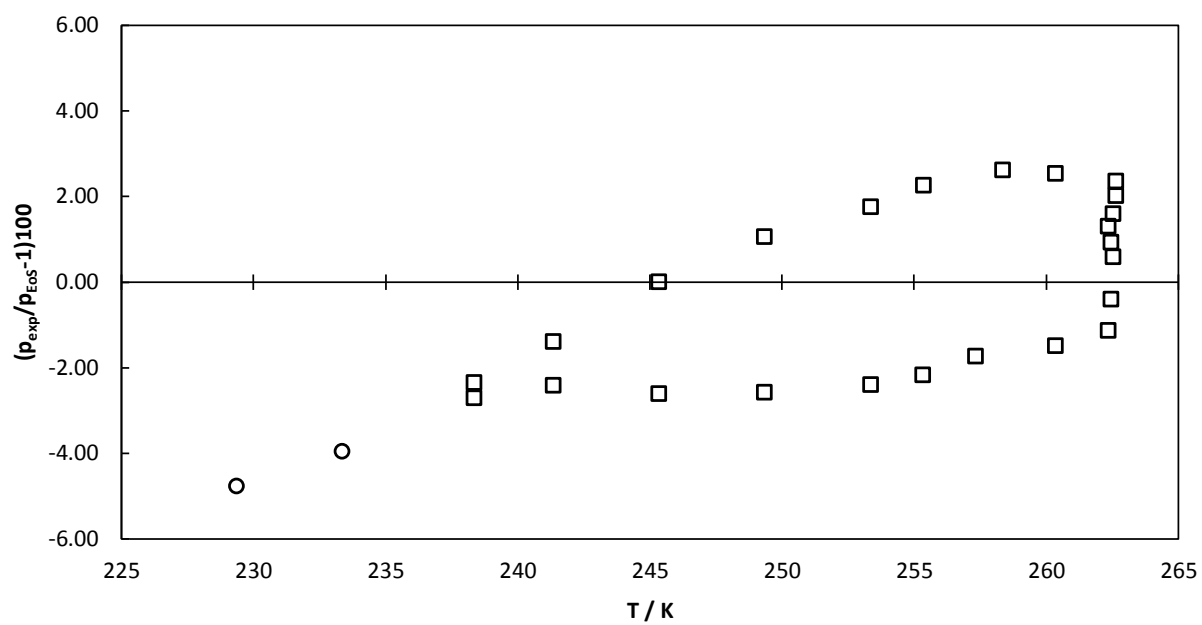

Figura 6.18 Desviaciones relativas respecto Peng-Robinson [17] empleando las reglas de mezcla de WongSandler [18] para el sistema $\left(\mathrm{x}_{1}\right) \mathrm{CO}_{2}+\left(1-\mathrm{x}_{1}\right) \mathrm{N}_{2}\left(\mathrm{x}_{1}=0.49905\right)$, punto de burbuja (o) rocío ( $\left.\square\right)$ esta tesis doctoral.

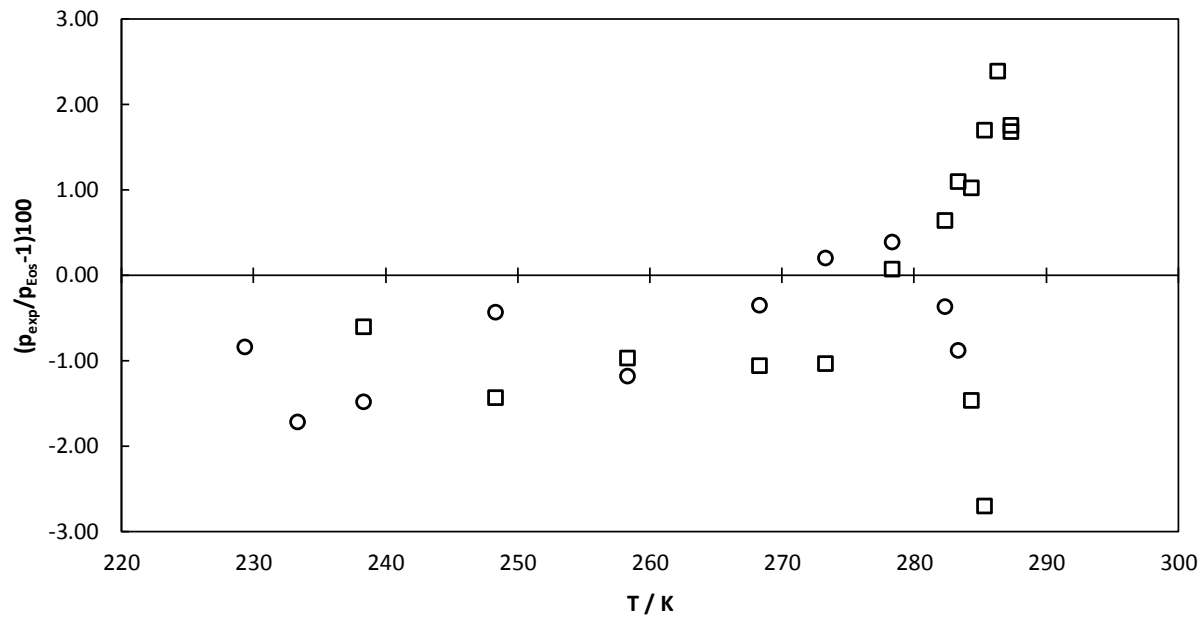

Figura 6.19 Desviaciones relativas respecto Peng-Robinson [17] empleando las reglas de mezcla de WongSandler [18] para el sistema para el sistema $\left(x_{1}\right) C O_{2}+\left(1-x_{1}\right) N_{2}\left(x_{1}=0.79777\right)$, punto de burbuja (o) rocío ( $\square$ ) esta tesis doctoral. 
Con esta nueva celda de medida, se ha logrado una disminución en la incertidumbre de medida con respecto al equipo anterior disponible en el laboratorio Gómez [15]. Aunque este equipo logró medir con mucha exactitud la curva de vaporización del $\mathrm{CO}_{2}$, presentaba problemas en la caracterización de mezclas. El diseño de la celda, donde el tubo que contenía la muestra, se encontraba sometido bajo compresión por las tapas de la cavidad, no garantizaba la estanqueidad, y se originaban microfugas.

Debido al diferente tamaño molecular de los componentes, las fugas provocan que la composición de la muestra se vea alterada. La falta de repetibilidad debida a la variación de la composición aumentaba sensiblemente la incertidumbre.

El diseño de la celda no permitía hacer vacío alrededor del tubo de cuarzo, el aire presente alteraba el factor de calidad de la señal. Este aire húmedo, podía condensar en la cavidad, modificando las frecuencias de resonancia.

El nuevo diseño presentado en esta tesis doctoral ha solventado estos problemas, el tubo de muestra se encuentra perfectamente centrado en el eje longitudinal de la cavidad, no está sometido a fuerzas de compresión que puedan provocar la ruptura del tubo. El sistema diseñado ofrece un mejor sello, capaz de soportar altas presiones, evitando las fugas en el equipo y logrando la repetibilidad en la medida.

La celda se ha construido con un nuevo material, cobre-zirconio al $15 \%$, que mejora la conductividad térmica y eléctrica, y se ha podido realizar vacío en el interior de la cavidad, alrededor del tubo, permitiendo mejorar el factor de calidad de la señal de microondas emitida, y, por consiguiente, ganando sensibilidad en la determinación del punto experimental, lo que se traduce en una menor incertidumbre en la medida. 

CAPítulo 7

Conclusiones 



\subsection{Introducción}

El principal objetivo de esta tesis doctoral ha sido el desarrollo de una técnica experimental, de la máxima exactitud, para la determinación de propiedades termodinámicas, en este caso, la envolvente de fase en la región del punto crítico. Se ha partido de una cavidad cilíndrica resonante en microondas propuesta por Gómez [15], a la que se le han realizado una serie de modificaciones y mejoras, que han incluido los planos de diseño, construcción en un taller mecánico, montaje de todos los elementos en el laboratorio, calibración de la instrumentación y validación con un compuesto test.

La sustancia seleccionada, cuyas propiedades termodinámicas son bien conocidas, ha sido el dióxido de carbono. La ecuación de estado Span \& Wagner [16] es capaz de predecir su comportamiento.

EL equipo ha sido automatizado con el fin de mejorar la toma de datos, esto se ha conseguido creando los programas para programar las rampas de presión y temperatura necesarias en cada punto experimental y que permiten relacionar la frecuencia de resonancia con la variación de presión en el cambio de fase de manera precisa.

El nuevo equipo ha conseguido mejorar la repetibilidad de las medidas y una reducción en la incertidumbre de medida.

Las medidas realizadas aportan datos experimentales para el estudio del equilibrio líquido vapor de sistemas binarios, formados por componentes de gases energéticos como el biogás, tanto en operaciones de almacenamiento, distribución y uso, como de purificación, eliminando el $\mathrm{CO}_{2}$ para su captura y almacenamiento. Estos componentes han sido $\mathrm{CO}_{2}, \mathrm{CH}_{4}, \mathrm{C}_{3} \mathrm{H}_{8}$ y $\mathrm{N}_{2}$. 
El trabajo realizado ha permitido alcanzar las siguientes conclusiones:

1. Se ha diseñado y construido una nueva cavidad cilíndrica resonante, partiendo de la experiencia adquirida con el equipo disponible en el laboratorio, mejorando la estanqueidad en el tubo de zafiro y una mayor calidad en la señal de resonancia, esto ha permitido reducir tanto los tiempos de medida como las incertidumbres.

2. Se ha determinado la curva de vaporización del $\mathrm{CO}_{2}$ desde $3.5 \mathrm{MPa}$ hasta el punto crítico, con una incertidumbre en la temperatura de $0.05 \mathrm{~K}$ y 0.006 MPa en presión. La desviación máxima respecto a la ecuación des estado de referencia de Span \& Wagner [16] ha sido inferior al $0.1 \%$ en presión e inferior a la incertidumbre de medida. Las coordenadas del punto crítico difieren en 0.04 K y 0.010 MPa de la ecuación de Span \& Wagner [16] con una incertidumbre expandida experimental de $0.06 \mathrm{~K}$ y $0.010 \mathrm{MPa}$ respectivamente.

3. Se ha determinado la envolvente de fases de tres sistemas binarios que contiene $\mathrm{CO}_{2},\left(\mathrm{CO}_{2}+\mathrm{CH}_{4}\right),\left(\mathrm{CO}_{2}+\mathrm{C}_{3} \mathrm{H}_{8}\right)$ y $\left(\mathrm{CO}_{2}+\mathrm{N}_{2}\right)$, en el rango de temperatura de $229.15 \mathrm{~K}$, a dos composiciones diferentes en fracción molar de $\mathrm{CO}_{2}$, hasta el punto de máxima temperatura (cricondentherm), característico de cada sistema y composición estudiada, incluida la zona de condensación retrógrada y el punto crítico.

4. Se ha realizado la modelización de estos sistemas empleando para ello la ecuación cúbica de estado de Peng-Robinson [17] empleando las reglas de mezcla de Wong-Sandler [18] y se ha realizado una comparación de los datos experimentales con la ecuación GERG 2008 [4], las diferencias promedio han sido para el sistema $(0.4) \mathrm{CO}_{2}+(0.6) \mathrm{CH}_{4}$ ha sido del $0.05 \%$, para el sistema $(0.6) \mathrm{CO}_{2}+(0.4) \mathrm{CH}_{4}$ del $0.27 \%$, para el sistema (0.6) $\mathrm{CO}_{2}+(0.4) \mathrm{C}_{3} \mathrm{H}_{8}$ del $0.03 \%$, para el sistema $(0.8) \mathrm{CO}_{2}+(0.2) \mathrm{C}_{3} \mathrm{H}_{8}$ del 
$0.04 \%$, para el sistema $(0.5) \mathrm{CO}_{2}+(0.5) \mathrm{N}_{2}$ del $0.08 \%$ y para el sistema $(0.8) \mathrm{CO}_{2}+(0.2) \mathrm{N}_{2}$ del $0.20 \%$. Para cada sistema se han realizado las comparaciones con la literatura existente.

5. Se han determinado de manera precisa todos los puntos de condensación retrógrada, a las isotermas seleccionadas, en cada uno de los sistemas estudiados. No se han encontrado puntos de condensación retrógrada en el sistema (0.8) $\mathrm{CO}_{2}+(0.2) \mathrm{C}_{3} \mathrm{H}_{8}$ debido a que la temperatura del punto crítico y el punto cricondentherm, $310.45 \mathrm{~K}$ y $310.71 \mathrm{~K}$ respectivamente se encuentran muy próximos.

6. Se ha realizado un estudio exhaustivo de las contribuciones a la incertidumbre de cada uno de los sistemas estudiados obteniendo las incertidumbres combinadas expandidas $(k=2)$ en la medida de la temperatura y la presión tanto en los puntos de burbuja como en los de rocío. Para el sistema (0.4) $\mathrm{CO}_{2}+(0.6) \mathrm{CH}_{4}$ han sido del $\mathrm{U}(\mathrm{T})=0.05 \mathrm{~K}, \mathrm{y} \mathrm{U}(\mathrm{p})$ $=0.010 \mathrm{MPa}$, para el sistema (0.6) $\mathrm{CO}_{2}+(0.4) \mathrm{CH}_{4}$ han sido del $\mathrm{U}(\mathrm{T})=0.05 \mathrm{~K}$, $\mathrm{y} U(\mathrm{p})=0.010 \mathrm{MPa}$, para el sistema $(0.6) \mathrm{CO}_{2}+(0.4) \mathrm{C}_{3} \mathrm{H}_{8}$ han sido del $\mathrm{U}(\mathrm{T})=$ $0.05 \mathrm{~K}, \mathrm{y} U(\mathrm{p})=0.010 \mathrm{MPa}$, para el sistema $(0.8) \mathrm{CO}_{2}+(0.2) \mathrm{C}_{3} \mathrm{H}_{8}$ han sido del $\mathrm{U}(\mathrm{T})=0.05 \mathrm{~K}, \mathrm{y} U(\mathrm{p})=0.010 \mathrm{MPa}$, para el sistema $(0.5) \mathrm{CO}_{2}+(0.5) \mathrm{N}_{2}$ han sido del $U(T)=0.05 \mathrm{~K}, \mathrm{y} U(\mathrm{p})=0.010 \mathrm{MPa}$ y para el sistema $(0.8) \mathrm{CO}_{2}+(0.2) \mathrm{N}_{2}$ han sido del $U(T)=0.05 \mathrm{~K}, \mathrm{y} U(\mathrm{p})=0.010 \mathrm{MPa}$.

7. Finamente y a modo de resumen, se ha aportado en esta tesis un conocimiento de las envolventes de fase de sistemas binarios de componentes de gases energéticos y se ha desarrollado una técnica experimental capaz de proporcionar datos exactos del equilibrio líquido vapor a altas presiones. 



\section{Bibliografía}



[1] United Nations, Framework Convention on Climate, (2015) 31.

[2] R.K. Pachauri, L. Meyer, Intergovernmental Panel on Climate Change, Clim. Chang. Synth. Rep. (2014) 2-26. doi:10.1088/1751-8113/44/8/085201.

[3] B. Page, The global status of CCS 2014, Summ. Rep. (2016) 1-28.

[4] O. Kunz, W. Wagner, The GERG-2008 wide-range equation of state for natural gases and other mixtures: An expansion of GERG-2004, J. Chem. Eng. Data. 57 (2012) 3032-3091. doi:10.1021/je300655b.

[5] R. Privat, J.N. Jaubert, Comments on "PVTxy properties of $\mathrm{CO}_{2}$ mixtures relevant for $\mathrm{CO}_{2}$ capture, transport and storage: Review of available experimental data and theoretical models," Appl. Energy. 93 (2012) 750752. doi:10.1016/j.apenergy.2011.09.012.

[6] B. Flach, K. Bendz, R. Krautgartner, S. Lieberz, M.E. Smith, EU Biofuels Annual, Glob. Agric. Inf. Netw. (2014) 1-34.

[7] B. Flach, S. Lieberz, M. Rondon, B. Williams, C. Teiken, EU Biofuels Annual, Glob. Agric. Inf. Netw. (2015) 1-42.

[8] T. Abbasi, S.M. Tauseef, S.A. Abbasi, Biogas energy, 2012. doi:10.1007/9781-4614-1040-9.

[9] EurObserv'ER, Biogas Barometer, (2017).

[10] W.J. Rogers, J.C. Holste, P.T. Eubank, K.R. Hall, Microwave apparatus for phase transition studies of corrosive fluids to $1.7 \mathrm{kbar}$ and $588 \mathrm{~K}$, Rev. Sci. Instrum. 56 (1985) 1907-1912. doi:10.1063/1.1138443.

[11] F. Fogh, P. Rasmussen, Detection of high-pressure dew and bubble points using a microwave technique, Ind. Eng. Chem. Res. 28 (1989) 371-375. doi:10.1021/ie00087a018.

[12] M.D. Frørup, J.T. Jepsen, A. Fredenslund, P. Rasmussen, High pressure dew and bubble points from microwave measurements, Fluid Phase Equilib. 52 (1989) 229-235. doi:10.1016/0378-3812(89)80329-2.

[13] A.R.H. Goodwin, M.D. Frørup, E.H. Stenby, Microwave detection of dew 
points: results for complex mixtures, J. Chem. Thermodyn. 23 (1991) 713715. doi:10.1016/S0021-9614(05)80189-2.

[14] A.R.H. Goodwin, J.B. Mehl, M.R. Moldover, Reentrant radio-frequency resonator for automated phase-equilibria and dielectric measurements in fluids, Rev. Sci. Instrum. 67 (1996) 4294-4303. doi:10.1063/1.1147580.

[15] A. Gómez, Desarrollo de una nueva técnica experimental de medida de equilibrio de fases a alta presión basada en una cavidad cilíndrica en microondas con aplicación al biogás, Tesis Doctoral. Universidad de Valladolid, 2015.

[16] R. Span, W. Wagner, A new equation of state for carbon dioxide covering the fluid region from the triple-point temperature to $1100 \mathrm{~K}$ at pressures up to $800 \mathrm{MPa}$, J. Phys. Chem. Ref. Data. 25 (1996) 1509-1596. doi:10.1063/1.555991.

[17] D.Y. Peng, D.B. Robinson, A new two-constant equation of state, Ind. Eng. Chem. Fundam. 15 (1976) 59-64. doi:10.1021/i160057a011.

[18] D.S.H. Wong, S.I. Sandler, A theoretically correct mixing rule for cubic equations of state, AIChE J. 38 (1992) 671-680. doi:10.1002/aic.690380505.

[19] J. Committee for Guides in Metrology, Evaluación de datos de medición. Guía para la expresión de la incertidumbre de medida, (2012) 1-142. doi:10.1515/ci.2013.35.2.22.

[20] NIST, NIST standard reference database 23: reference fluid thermodynamic and transport properties (REFPROP), Version 9.1, (2013). doi:10.1234/12345678.

[21] A. Harvey, E. Lemmon, Method for estimating the dielectric constant of natural gas mixtures, Int. J. Thermophys. 26 (2005) 31-46. doi:10.1007/s10765-005-2351-5.

[22] H.D. Young, R.A. Freedman, A.L. Ford, Física Universitaria Vol.1, Pearson Educ. (2009) 760. 
[23] H.D. Young, R.A. Freedman, A.L. Ford, Física Universitaria Vol.2, Pearson Educ. 2 (2009) 708.

[24] J.W. Gibbs, The scientific papers of J Willard Gibbs, Nature. 75 (1907) 361362. doi:10.1038/075361a0.

[25] G.N. Lewis, M. Randall, Thermodynamics and the free energy of chemical substances, Mc Graw-Hill, 1923.

[26] J.M. Prausnitz, Molecular thermodynamics of fluid-phase equilibria, Prentice Hall. (1969) 158-159. doi:10.1016/0021-9614(70)90078-9.

[27] J.M.S. Fonseca, R. Dohrn, S. Peper, High-pressure fluid-phase equilibria: Experimental methods and systems investigated (2005-2008), Fluid Phase Equilib. 300 (2011) 1-69. doi:10.1016/j.fluid.2010.09.017.

[28] P.H.V. Konynenburg, R.L. Scott, Critical lines and phase equilibria inbBinary Van Der Waals mixtures, Philos. Trans. R. Soc. A Math. Phys. Eng. Sci. 298 (1980) 495-540. doi:10.1098/rsta.1980.0266.

[29] E.D. Silverman, G. Thodos, Cricondentherms and cricondenbars their prediction for binary hydrocarbon systems, Ind. Eng. Chem. Fundam. 1 (1962) 299-303. doi:10.1021/i160004a013.

[30] M.B. King, Phase equilibrium in mixtures, Elsevier Science, 1969.

[31] L.F. Chen, C.K. Ong, C.P. Neo, V. V. Varadan, V.K. Varadan, Microwave Electronics. Measurement and Materials Characterization, John Wiley \& , Ltd, 2004. doi:10.1002/0470020466.

[32] A.P. Gregory, R.N. Clarke, A review of RF and microwave techniques for dielectric measurements on polar liquids, IEEE Trans. Dielectr. Electr. Insul. 13 (2006) 727-743. doi:10.1109/TDEI.2006.1667730.

[33] L.R. Victoriano, M.L.M. del Mar, C.P. Castro, Electromagnetismo II, Universidad Nacional de Educación a Distancia Madrid, 2013.

[34] A. Tamburrano, D. Desideri, A. Maschio, S.S. Maria, Coaxial waveguide methods for shielding effectiveness measurement of planar materials up to 
$18 \mathrm{GHz}$, IEEE Trans. Electromagn. Compat. 56 (2014) 1386-1395. doi:10.1109/TEMC.2014.2329238.

[35] Agilent Technologies, Understanding the fundamental principles of vector network analysis, Appl. Note 5965-7707E. (2012) 15. http://www.agilent.com/.

[36] P. Atkins, J. De Paula, Physical chemistry, W.H. Freeman nd Company, 2009. doi:10.1021/ed056pA260.1.

[37] R.E. Fornari, P. Alessi, I. Kikic, High pressure fluid phase equilibria: Experimental methods and systems investigated (1978-1987), Fluid Phase Equilib. 57 (1990) 1-33. doi:10.1016/0378-3812(90)80010-9.

[38] M. Christov, R. Dohrn, High-pressure fluid phase equilibria: Experimental methods and systems investigated (1994-1999), Fluid Phase Equilib. 202 (2002) 153-218. doi:10.1016/S0378-3812(02)00096-1.

[39] R. Dohrn, S. Peper, J.M.S. Fonseca, High-pressure fluid-phase equilibria: Experimental methods and systems investigated (2000-2004), Fluid Phase Equilib. 288 (2010) 1-54. doi:10.1016/j.fluid.2009.08.008.

[40] E.F. May, Measurement of the thermodynamic properties of single phases. IUPAC Experimental Thermodynamics, Volume VI, 2003. doi:10.1021/je600558y.

[41] D.S. Tsiklis, Handbook of techniques in high pressure research and engineering, plenum press, 1968. doi:10.1007/978-1-4684-8360-4.

[42] R.. Weir, T.W. De Loos, Measurement of the thermodynamic properties of multiple phases, Experimental Thermodynamics vol VII, Elsevier Ltd, 2005.

[43] M.N. Afsar, J.R. Birch, R.N. Clarke, G.W. Chantry, The measurement of the properties of materials, Proc. IEEE. 74 (1986) 183-199. doi:10.1109/PROC.1986.13432.

[44] J. Asmussen, R. Mallavarpu, J.R. Hamann, The design of a microwave plasma cavity, Proc. IEEE. 62 (1974) 109-117. doi:10.1109/PROC.1974.9391. 
[45] B.A. Younglove, G.C. Straty, A capacitor for accurate wide range dielectric constant measurements on compressed fluids, Rev. Sci. Instrum. 41 (1970) 1087-1089. doi:10.1063/1.1684699.

[46] G.C. Straty, B.A. Younglove, Compact capacitor for wide-range dielectric constant measurements on small amounts of fluids, Rev. Sci. Instrum. 50 (1979) 1309-1310. doi:10.1063/1.1135702.

[47] A.R.H. Goodwin, M.R. Moldover, Phase border and density determinations in the critical region of (carbon dioxide + ethane) determined from dielectric permittivity measurements, J. Chem. Thermodyn. 29 (1997) 1481-1494. doi:10.1006/jcht.1997.0257.

[48] E.F. May, T.J. Edwards, A.G. Mann, C. Edwards, R.C. Miller, Development of an automated phase behaviour measurement system for lean hydrocarbon fluid mixtures, using re-entrant rf/microwave resonant cavities, Fluid Phase Equilib. 185 (2001) 339-347. doi:10.1016/S0378-3812(01)00482-4.

[49] E.F. May, T.J. Edwards, A.G. Mann, C. Edwards, An improved microwave apparatus for phase behaviour measurements in lean gas condensate fluids, Fluid Phase Equilib. $215 \quad$ (2004) 245-252. doi:10.1016/j.fluid.2003.08.015.

[50] E.F. May, T.J. Edwards, A.G. Mann, C. Edwards, Dew point, liquid volume, and dielectric constant measurements in a vapor mixture of methane + propane using a microwave apparatus, Int. J. Thermophys. 24 (2003) 15091525.

[51] P.H. Huang, D.C. Ripple, M.R. Moldover, G.E. Scace, A Reference standard for measuring humidity of air using a re-entrant radio frequency resonator, Natl. Inst. Stand. Technol. Gaithersburg, Maryland, U.S.A. (2006) 2-5.

[52] M.E. Kandil, K.N. Marsh, A.R.H. Goodwin, A re-entrant resonator for the measurement of phase boundaries: Dew points for $\left\{0.4026 \mathrm{CH}_{4}+0.5974\right.$ $\left.\mathrm{C}_{3} \mathrm{H}_{8}\right\}$, J. Chem. Thermodyn. 37 (2005) 684-691. 
doi:10.1016/j.jct.2004.11.004.

[53] M.E. Kandil, K.N. Marsh, A.R.H. Goodwin, Determination of the relative permittivity and density within the gas phase and liquid volume fraction formed within the two-phase region for $\left(0.4026 \mathrm{CH}_{4}+0.5974 \mathrm{C}_{3} \mathrm{H}_{8}\right)$ with a radio frequency re-entrant cavity, J. Chem. Eng. Data. 52 (2007) 1660-1671. doi:10.1021/je700053u.

[54] R.J. Underwood, R. Cuccaro, S. Bell, R.M. Gavioso, D.M. Ripa, M. Stevens, M. De Podesta, A microwave resonance dew-point hygrometer, Meas. Sci. Technol. 23 (2012) 1-9. doi:10.1088/0957-0233/23/8/085905.

[55] M.B. Ewing, D.D. Royal, A highly stable cylindrical microwave cavity resonator for the measurement of the relative permittivities of gases, J. Chem. Thermodyn. 34 (2002) 1073-1088. doi:10.1006/jcht.2002.0962.

[56] B. Kapilevich, S.G. Ogourtsov, V.G. Belenky, A.B. Maslenikov, A.S. Omar, Accurate microwave resonant method for complex permittivity measurements of liquids, IEEE Trans. Microw. Theory Tech. 48 (2000) 21592164. doi:10.1109/22.884209.

[57] B. Kapilevich, B. Litvak, Optimized microwave sensor for online concentration measurements of binary liquid mixtures, IEEE Sens. J. 11 (2011) 2611-2616. doi:10.1109/JSEN.2011.2149517.

[58] H. Kawabata, H. Tanpo, R. Members, An improvement of the perturbation method using a TM 010 mode cylindrical cavity, IEICE Trans. Electron. E86 (2003) 2371-2378.

[59] A.J. Estin, E. Bussey, Errors in dielectric measurements due to a sample insertion hole in a Cavity, IRE Trans. Microw. Theory Tech. 8 (1960) 650653.

[60] H. Kawabata, H. Tanpo, Y. Kobayashi, A rigorous analysis of a TM010 mode cylindrical cavity to measure accurate complex permittivity of liquid, in: 33rd Eur. Microw. Conf. 2003, 2003. doi:10.1109/EUMA.2003.341064. 
[61] H. Kawabata, Y. Kobayashi, Accurate measurements of complex permittivity of liquid based on a TM 1010 mode cylindrical cavity method, 2005 Eur. Microw. Conf. 1 (2005) 369-372.

[62] P. Kanpan, E. Khansalee, N. Puangngernmak, S. Chalermwisutkul, TM010 mode cylindrical cavity for complex permittivity measurement of liquid using field analysis technique, in: 2012 9th Int. Conf. Electr. Eng. Comput. Telecommun. Inf. Technol., 2012: pp. 1-4. doi:10.1109/ECTICon.2012.6254274.

[63] D.M. Pozar, Microwave Engineering four edition, John Wiley \& Sons, 2012. doi:10.1080/01422419908228843.

[64] G. Birnbaum, J. Franeau, Measurement of the dielectric constant and loss of solids and liquids by a cavity perturbation method, J. Appl. Phys. 20 (1949) 817-818. doi:10.1063/1.1698535.

[65] P. Russer, Electromagnetics, Microwave Circuits and Antenna Design for Communications Engineering (Antennas \& Propagation Library), 2014. doi:10.1128/AAC.03728-14.

[66] P.J. Petersan, S.M. Anlage, Measurement of resonant frequency and quality factor of microwave resonators: Comparison of methods, J. Appl. Phys. 84 (1998) 3392-3402. doi:10.1063/1.368498.

[67] K.J. Coakley, J.D. Splett, M.D. Janezic, R.F. Kaiser, Estimation of Q -Factors and Resonant Frequencies, IEEE Trans. Microw. Theory Tech. 51 (2003) 862-868. doi:10.1109/TMTT.2003.808578.

[68] E. Martin, A. Hernandez, M.C. Sanchez, J.M. Zamarro, J. Margineda, Automatic measurement of $Q$ factor and resonant frequency of microwave resonators, J. Phys. E. 14 (1981) 961-962. doi:10.1088/0022$3735 / 14 / 8 / 016$.

[69] R. Feynman, The Feynman lectures on physics. California Institute of Technology, (2016). http://www.feynmanlectures.caltech.edu/I_49.html. 
[70] Peter Barlow, An elementary investigation of the theory of numbers, J.Johnson and Co, 1811.

[71] H. Preston-Thomas, The international temperature scale of 1990 (ITS-90), Metrología. 27 (1990) 3-10. doi:10.1088/0026-1394/27/1/002.

[72] J.H. Kim, M.S. Kim, Vapor-liquid equilibria for the carbon dioxide + propane system over a temperature range from 253.15 to $323.15 \mathrm{~K}$, Fluid Phase Equilib. 239 (2005) 13-19. doi:10.1016/j.fluid.2005.09.006.

[73] B. Saleh, M. Wendland, Measurement of vapor pressures and saturated liquid densities of pure fluids with a new apparatus, J. Chem. Eng. Data. 50 (2005) 429-437. doi:10.1021/je0497496.

[74] L. Gil, S.T. Blanco, C. Rivas, E. Laga, J. Fernández, M. Artal, I. Velasco, Experimental determination of the critical loci for $\left\{n-C_{6} \mathrm{H}_{14}\right.$ Or $\mathrm{CO}_{2}+$ alkan-1ol\} mixtures. Evaluation of their critical and subcritical behavior using PC$\begin{array}{llllll}\text { SAFT EOS, J. Supercrit. Fluids. } 71 & \text { (2012) 26-44. }\end{array}$ doi:10.1016/j.supflu.2012.07.008.

[75] Y. Suehiro, M. Nakajima, K. Yamada, M. Uematsu, Critical parameters of $\left\{\mathrm{xCO}_{2}+(1-\mathrm{x}) \mathrm{CHF}_{3}\right\}$ for $\mathrm{x}=(1.0000,0.7496,0.5013$, and 0.2522$)$, J. Chem. Thermodyn. 28 (1996) 1153-1164.

[76] A. Diefenbacher, M. Crone, M. Turk, Critical properties of $\mathrm{CO}_{2}, \mathrm{CHF}_{3}, \mathrm{SF}_{6}$, $\left(\mathrm{CO}_{2}+\mathrm{CHF}_{3}\right)$, and $\left(\mathrm{CHF}_{3}+\mathrm{SF}_{3}\right)$, J. Chem. Thermodyn. 30 (1998) 481-496.

[77] H. Renon, J.M. Prausnitz, Local compositions in thermodynamic excess functions for liquid mixtures, AIChE J. 14 (1968) 135-144. doi:10.1002/aic.690140124.

[78] J.A. Nelder, R. Mead, A simplex method for function minimization, Comput. J. 7 (1965) 308-313. doi:10.1093/comjnl/7.4.308.

[79] Á. Martín, M.D. Bermejo, F.A. Mato, M.J. Cocero, Teaching advanced equations of state in applied thermodynamics courses using open source programs, Educ. Chem. Eng. 6 (2011) e114-e121. 
doi:10.1016/j.ece.2011.08.003.

[80] H.G. Donnelly, D.L. Katz, Phase equilibria in the carbon dioxide-methane system, Ind. Eng. Chem. 46 (1954) 511-517.

[81] M.S.W. Wei, T.S. Brown, A.J. Kidnay, E.D. Sloan, Vapor + liquid equilibria for the ternary system methane + ethane + carbon dioxide at $230 \mathrm{~K}$ and its constituent binaries at temperatures from 207 to 270 K, J. Chem. Eng. Data. 40 (1995) 726-731. doi:10.1021/je00020a002.

[82] L.A. Webster, A.J. Kidnay, Vapor-liquid equilibria for the methane-propanecarbon dioxide systems at $230 \mathrm{~K}$ and $270 \mathrm{~K}$, J. Chem. Eng. Data. 46 (2001) 759-764. doi:10.1021/je000307d.

[83] T.A. Al-Sahhaf, A.J. Kidnay, E.D. Sloan, Liquid + vapor equilibria in the $\mathrm{N}_{2}+$ $\mathrm{CO}_{2}+\mathrm{CH}_{4}$ system, Ind. Eng. Chem. Fundam. 22 (1983) 372-380.

[84] J. Davalos, W.R. Anderson, R.E. Phelps, A.J. Kidnay, Liquid-vapor equilibria at $250.00 \mathrm{~K}$ for systems containing methane, ethane, and carbon dioxide, J. Chem. Eng. Data. 21 (1976) 81-84. doi:10.1021/je60068a030.

[85] V.G. Niesen, J.C. Rainwater, Critical locus, (vapor + liquid) equilibria, and coexisting densities of (carbon dioxide + propane) at temperatures from $311 \mathrm{~K}$ to $361 \mathrm{~K}$, J. Chem. Thermodyn. 22 (1990) 777-795. doi:10.1016/00219614(90)90070-7.

[86] W.W. Akers, R.E. Kelley, T.G. Lipscomb, Carbon-dioxide propane system, Ind. Eng. Chem. 46 (1954) 2535-2536. doi:10.1021/ie50540a039.

[87] S.E.M. Hamam, B.C.Y. Lu, Isothermal vapor-liquid equilibria in binary system propane-carbon dioxide, J. Chem. Eng. Data. 21 (1976) 200-204. doi:10.1021/je60069a020.

[88] T.S. Brown, V.G. Niesen, E.D. Sloan, A.J. Kidnay, Vapor-liquid equilibria for the binary systems of nitrogen, carbon dioxide, and n-butane at temperatures from 220 to 344 K, Fluid Phase Equilib. 53 (1989) 7-14. doi:10.1016/0378-3812(89)80067-6. 
[89] O. Fandiño, J.P.M. Trusler, D. Vega-Maza, Phase behavior of $\left(\mathrm{CO}_{2}+\mathrm{H}_{2}\right)$ and $\left(\mathrm{CO}_{2}+\mathrm{N}_{2}\right)$ at temperatures between (218.15 and 303.15) $\mathrm{K}$ at pressures up to $15 \mathrm{MPa}$, Int. J. Greenh. Gas Control. 36 (2015) 78-92. doi:10.1016/j.ijggc.2015.02.018.

[90] S.F. Westman, H.G.J. Stang, S.W. Løvseth, A. Austegard, I. Snustad, S.T. Størset, I.S. Ertesvåg, Vapor-liquid equilibrium data for the carbon dioxide and nitrogen $\left(\mathrm{CO}_{2}+\mathrm{N}_{2}\right)$ system at the temperatures 223, 270, 298 and 303 $\mathrm{K}$ and pressures up to $18 \mathrm{MPa}$, Fluid Phase Equilib. 409 (2016) 207-241. doi:10.1016/j.fluid.2015.09.034. 
Anexos 

Anexo I: Diagramas y planos de la celda de medida

En este anexo, se incluyen los planos técnicos de los componentes principales que conforman la celda de medida, Cavidad principal, cubiertas, transducer y piezas mecanizadas para el correcto funcionamiento de la cavidad. Todos los detalles constructivos, así como los materiales empleados para su construcción se encuentran descritos en el apartado 4.2 de la presente memoria. 


\section{Material: Luvata ZrK015}
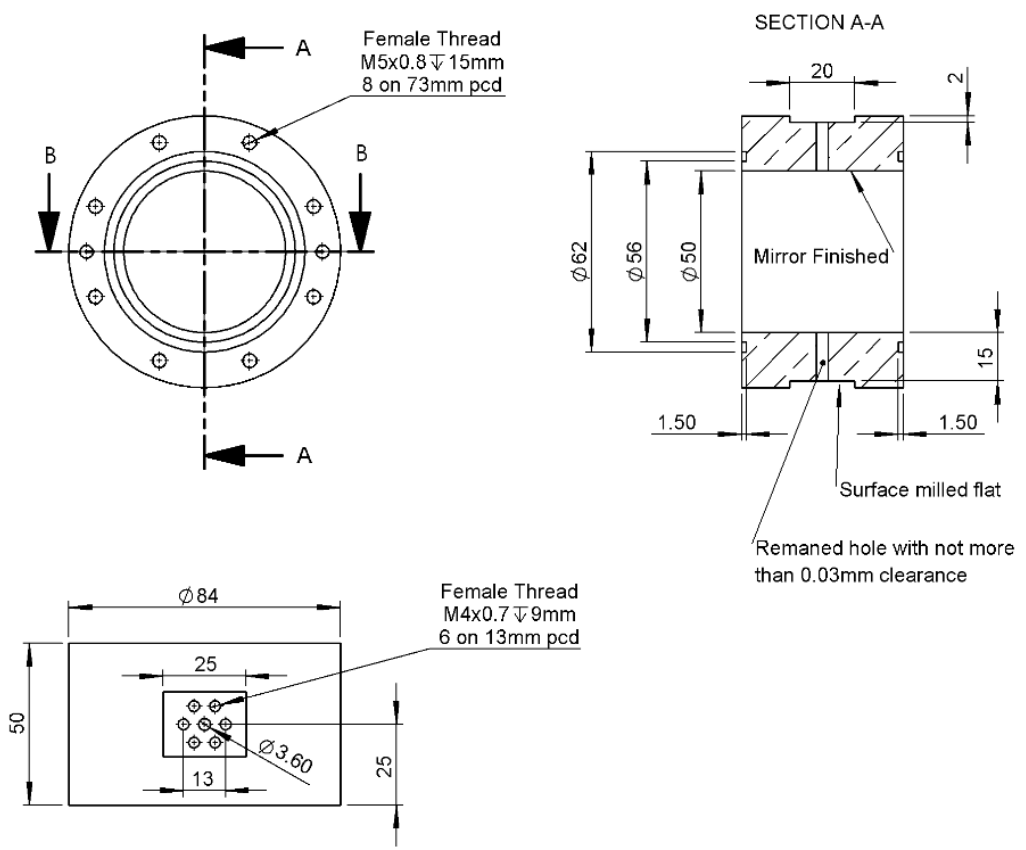

than $0.03 \mathrm{~mm}$ clearance

SECTION B-B

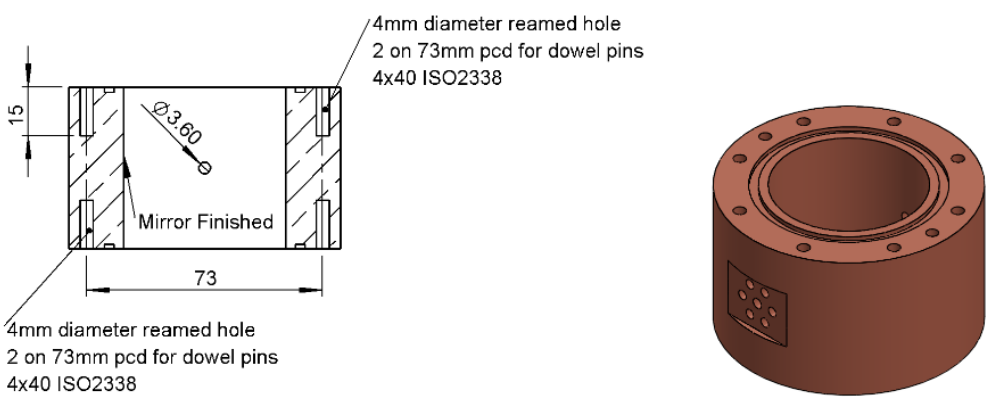

\section{termocal 8}

Authors:

Jose Juan Segovia Puras

Ángel Gómez Hernández

Rodrigo Susial Martín

cylindrical cavity microwave resonator

Name of plot

1. Central body

\begin{tabular}{c|c} 
Scale & Units \\
$1 / 2$ & $\mathrm{~mm}$
\end{tabular}

Plot number $\quad 1$ 


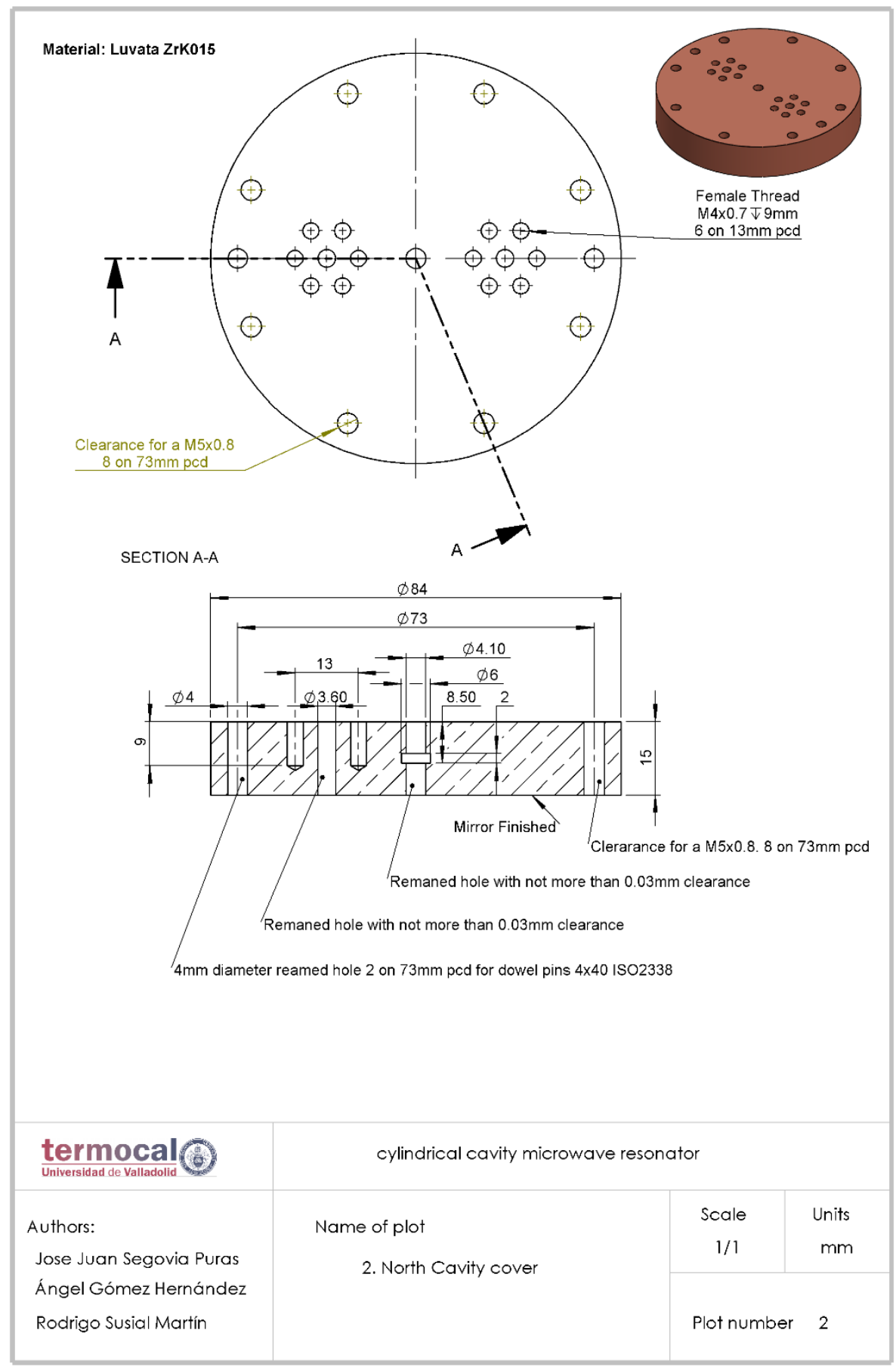




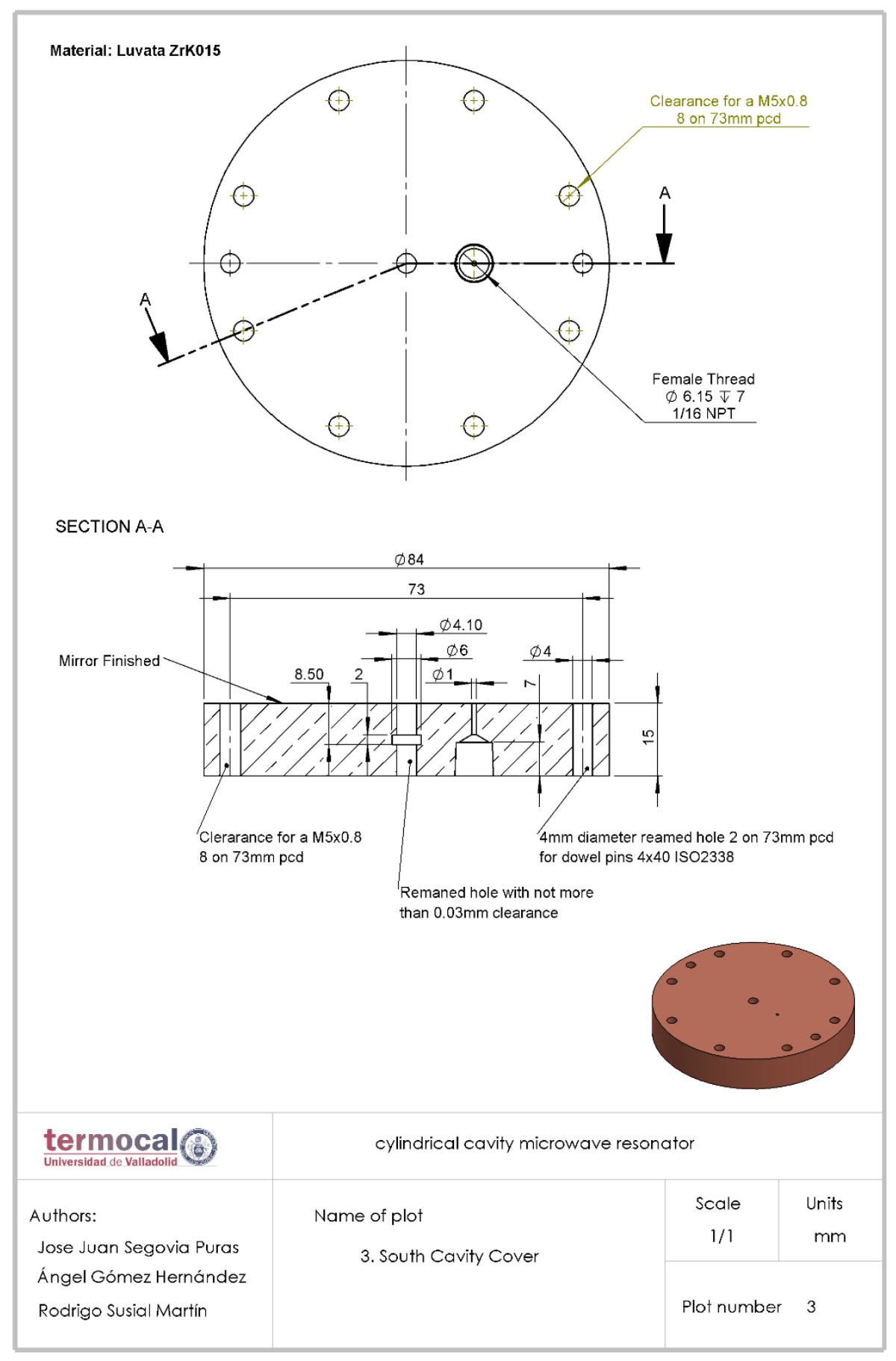


Material: Stainless Steal 316

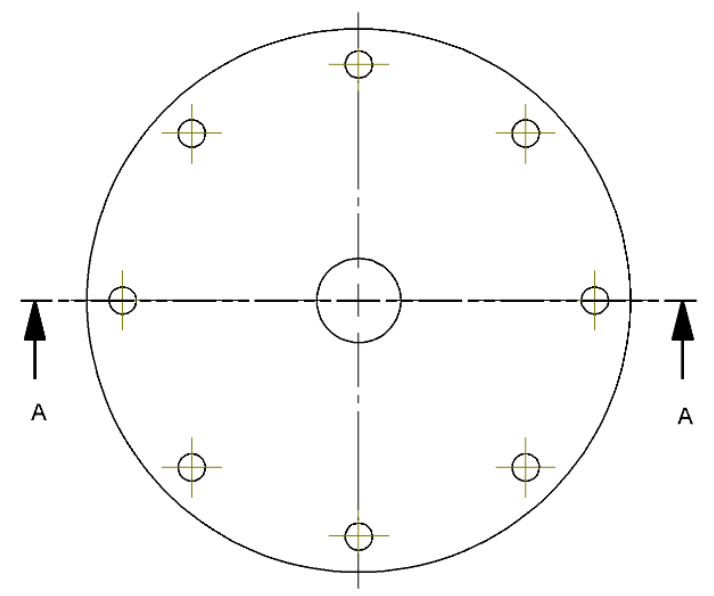

SECTION A-A
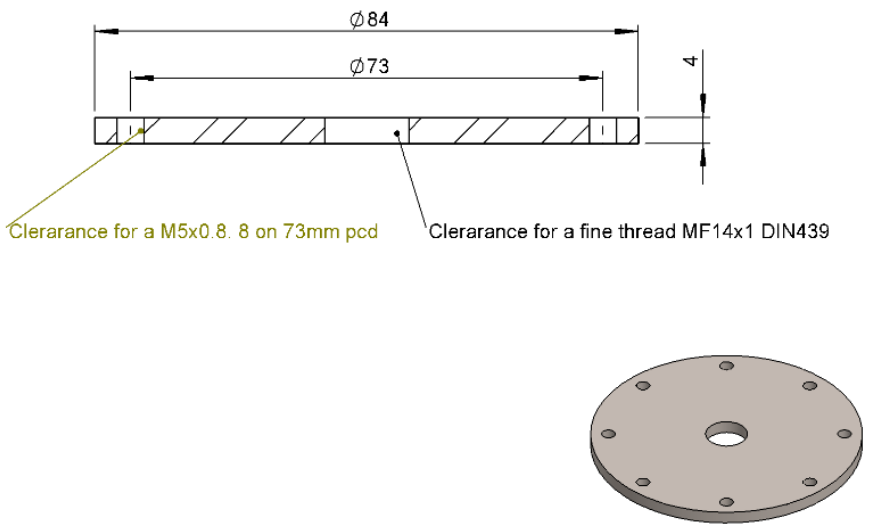

\section{termocal}

\section{Authors:}

Jose Juan Segovia Puras Ángel Gómez Hernández

Rodrigo Susial Martín cylindrical cavity microwave resonator
Name of plot

4. Stainless Steal circular plate
Scale

$1 / 1$

Units

$\mathrm{mm}$
Plot number $\quad 4$ 
Material: Stainless Steal 316
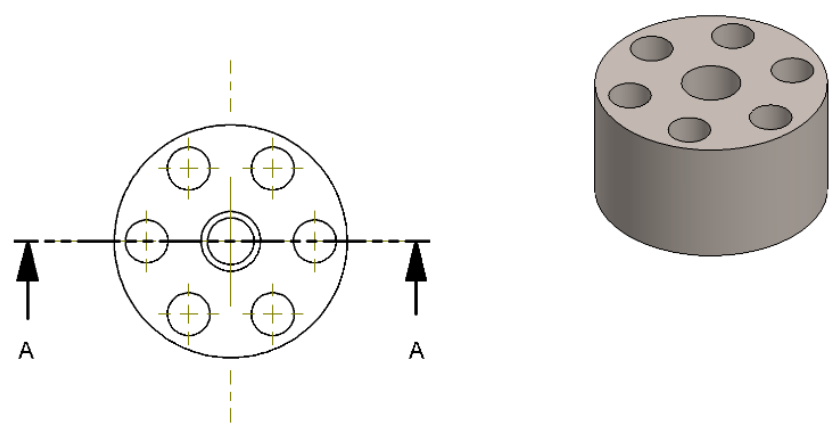

SECTION A-A

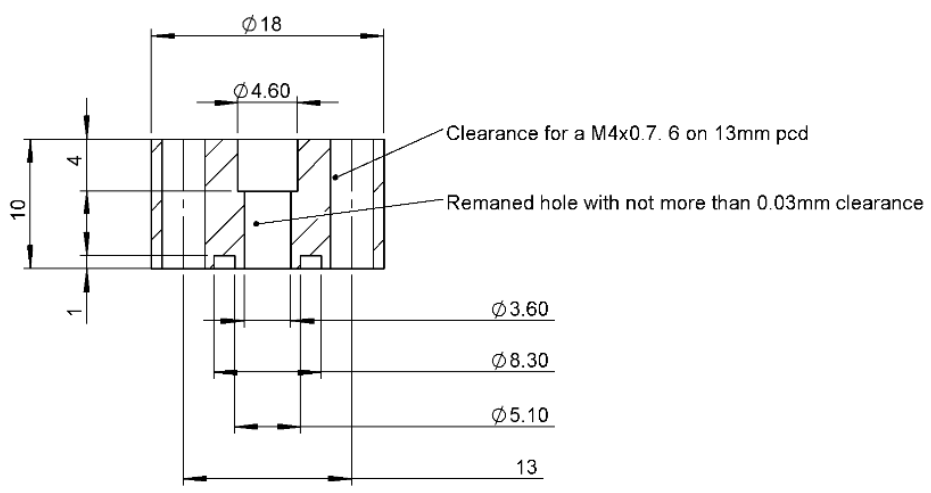

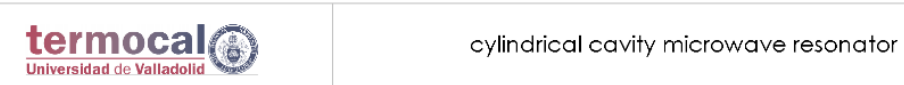

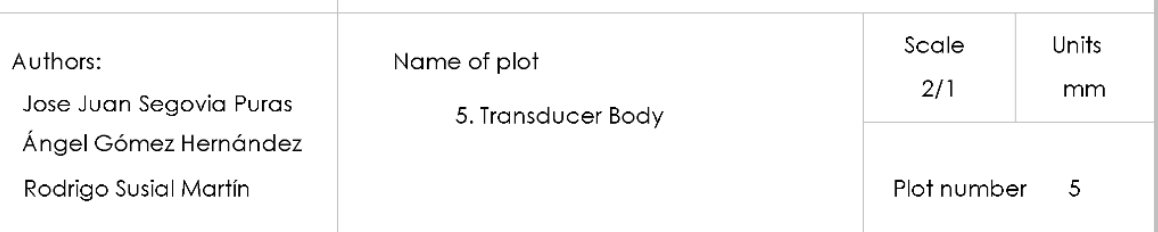


Material: Stainless Steal 316

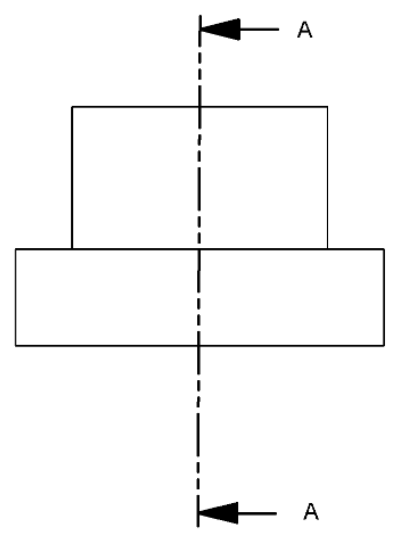

A

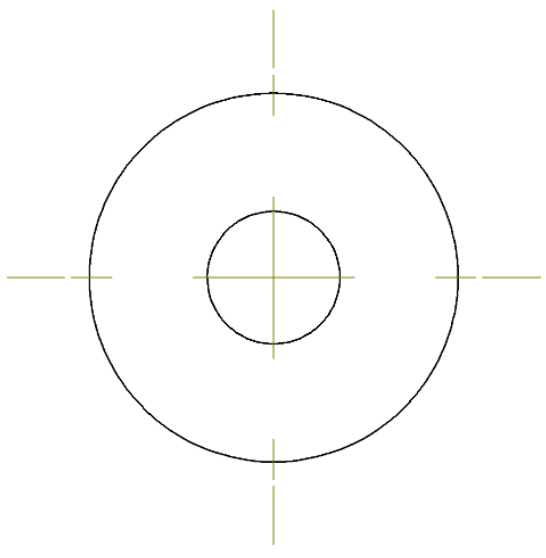

SECTION A-A

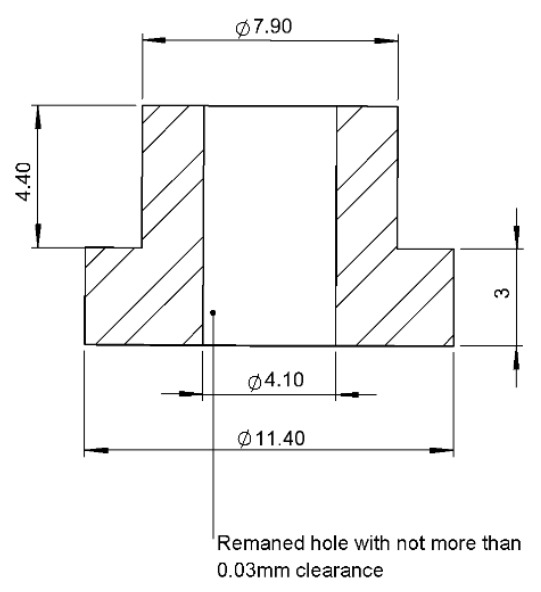

$0.03 \mathrm{~mm}$ clearance

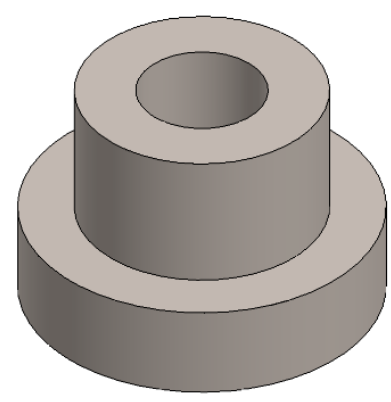

\section{$\frac{\text { termocal }}{\text { Universidad de Valladolid }}(6)$}

Authors:

Jose Juan Segovia Puras

Ángel Gómez Hernández

Rodrigo Susial Martín

\section{Cylindrical Cavity Microwave Resonator}

Name of plot

6. Special Body

\begin{tabular}{c|c}
$\begin{array}{c}\text { Scale } \\
2 / 1\end{array}$ & Units \\
& $\mathrm{mm}$ \\
& \\
Plot number & 6
\end{tabular}


Material: HIP model 316
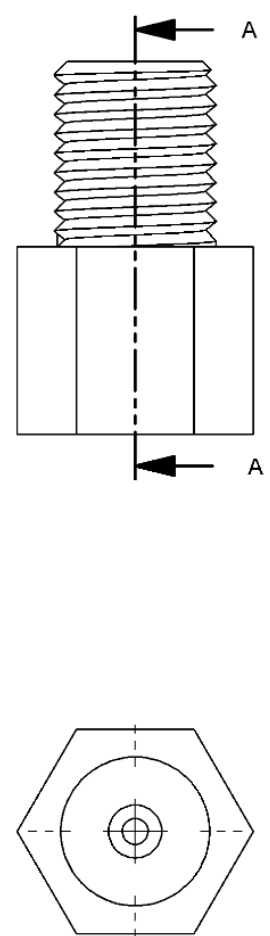
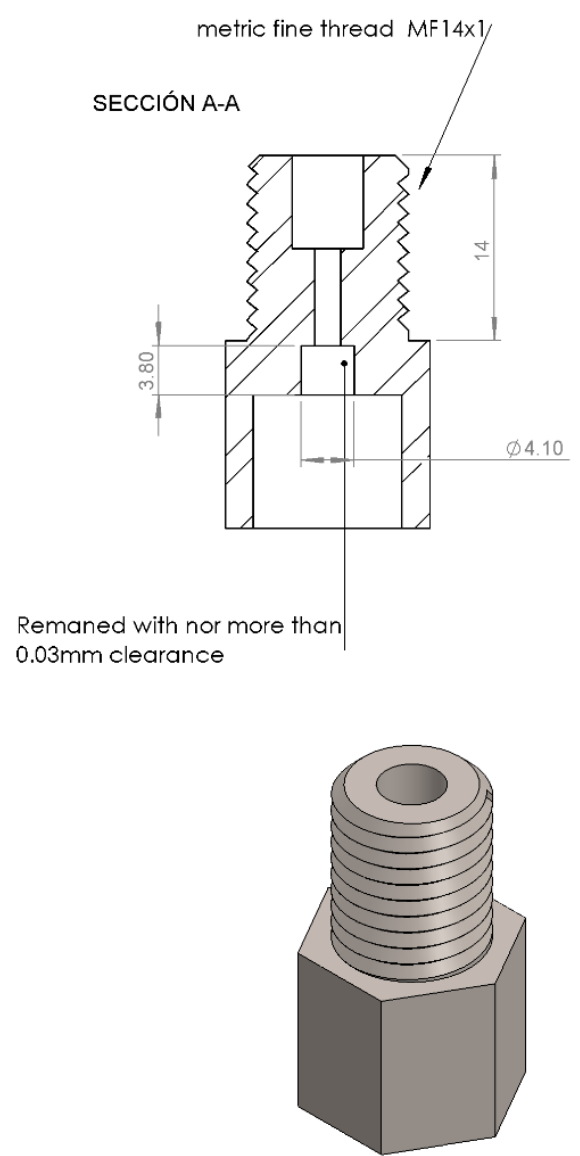

Hexagonal nut, low profile

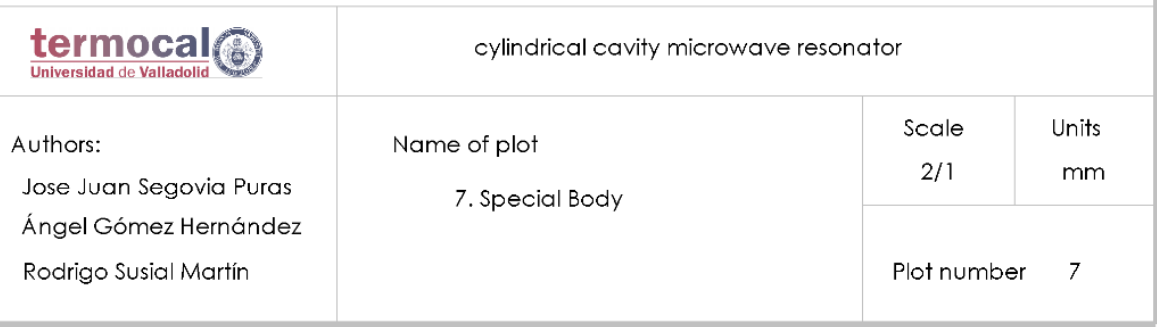



Universidad deValladolid

PROGRAMA DE DOCTORADO EN INGENIERÍA TERMODINÁMICA DE FLUIDOS

PhD THESIS:

\section{DETERMINATION OF PHASE DEVELOP OF BINARY \\ MIXTURES OF BIOGAS WITH A MICROWAVE RESONATOR}

A thesis submitted by Rodrigo Susial Martín, in partial fulfilment of the requirements for the degree of Doctor of Philosophy

Supervised by:

José Juan Segovia Puras. PhD

María del Carmen Martín Gonzalez. PhD

Valladolid, 2019 



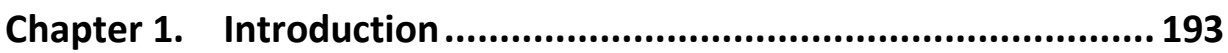

1.1 The environmental problem 193

1.2 State of the art 195

1.3 Thesis doctoral aims 197

1.4 Structure of the thesis 198

Chapter 2. Thermophysical properties........................................ 203

2.1 Introduction 203

2.2 Equilibrium properties 204

2.3 The chemical potential 204

2.4 Fugacity coefficient 205

2.5 Electromagnetic properties of fluids 206

2.6 Dielectric constant 207

2.7 Microwave network 207

2.8 Relationship between volumetric and dielectric properties 208

Chapter 3. Experimental techniques for high pressure equilibria determination

3.1 Introduction 213

3.2 Experimental techniques for high pressure equilibrium measurement

3.3 Experimental techniques for the measurement of dielectric properties

3.4 Cylindrical resonant cavity in microwave basic principle 216

Chapter 4. Experimental setup ................................................ 221

$\begin{array}{lll}4.1 & \text { Introduction } & 221\end{array}$

4.2 Cylindrical cavity resonator description 221 
$\begin{array}{lll}4.3 & \text { Experimental setup } & 222\end{array}$

$\begin{array}{lll}4.4 & \text { Control software } & 224\end{array}$

Chapter 5. $\mathrm{CO}_{2}$ measurements .............................................. 229

$\begin{array}{llr}5.1 & \text { Introduction } & 229\end{array}$

$\begin{array}{lll}5.2 & \text { Experimental procedure } & 229\end{array}$

5.3 Uncertainty Determination 230

$\begin{array}{lll}5.4 & \text { Experimental results } & 232\end{array}$

$\begin{array}{lll}5.5 & \text { Analysis of the results } & 237\end{array}$

Chapter 6. Phase behaviour and modelling of binary systems with $\mathrm{CO}_{2}$ 241

$\begin{array}{lll}6.1 & \text { Introduction } & 241\end{array}$

6.2 Gravimetric samples preparation 241

6.3 Experimental determination of the phase envelope 242

6.4 Uncertainties Determination 243

$\begin{array}{lll}6.5 & \text { Experimental results } & 244\end{array}$

$\begin{array}{lll}6.6 & \text { Analysis of the results } 253\end{array}$

Chapter 7. Conclusions............................................................. 257

$\begin{array}{llr}7.1 & \text { Introduction } & 257\end{array}$

Bibliography .............................................................. 263 


\section{CHAPTER 1}

\section{Introduction}





\section{Chapter 1. Introduction}

\subsection{The environmental problem}

To deny that global warming is mainly caused by the high concentrations of $\mathrm{CO}_{2}$ found in the atmosphere is to deny a reality that affects all beings on this planet. Already in Paris [1], an agreement endorsed by 195 countries was established whereby a global warming limit would be established well below $2^{\circ} \mathrm{C}$ above preindustrial levels. This agreement allows each country to establish its own plan of action in order to achieve this objective, in the form of nationally determined planned contributions to achieve the voluntary targets for reducing its anthropogenic greenhouse gas emissions.

$78 \%$ of greenhouse gases result from the combustion of fossil fuels and industrial processes [2]. Progressive industrial growth and growing global energy demand may increase the accumulation of carbon dioxide emissions in the atmosphere. Measures are therefore needed to drastically reduce these emissions. Reducing carbon dioxide emissions through carbon capture and storage (CCS) is a technology that is often discussed [3], however, there are still several technical challenges to the design and operation of CCS systems. $\mathrm{CO}_{2}$ captured in power plants and industry is compressed and piped, and then injected into the underground storage site. Typical temperature and pressure conditions of $\mathrm{CO}_{2}$ involved processes vary depending on needs, but under CCS conditions the operating range is from $0.05 \mathrm{MPa}$ to $20 \mathrm{MPa}$ and from $298.15 \mathrm{~K}$ to $620.00 \mathrm{~K}$. Suitable storage sites include deep saline aquifers with the highest storage capacity, followed by depleted oil and gas fields, and unachievable coal seams.

Reliable knowledge of the thermophysical properties of the substances involved in the processes is of crucial importance to ensure efficient and safe plant design and effective operation of the processes within the CCS chain. As process 
conditions cover a wide range of pressures, temperatures and complex mixtures of multicomponent fluids, it is not cost-effective to carry out experiments for every combination of parameters. Therefore, measuring thermophysical data of relatively simpler binary and ternary systems, for the usual pressure and temperature range, and using them to verify and improve predictive thermodynamic models, is a way to simplify and address the problem. With improved predictive thermodynamic models, data from various components can be used to test the model. Enhanced predictive thermodynamic models can be applied to improve process design, safety and optimization along the CCS chain.

Fundamental properties such as phase equilibrium of $\mathrm{CO}_{2}$ containing systems can be predicted with multiparametric equations such as the GERG 2008 equation of state [4], however, the accuracy of these models depends strongly on the reliability of the experimental data on which they are based [5], because of this, the precise measurement of thermodynamic properties of systems in which $\mathrm{CO}_{2}$ capture and storage is involved is more than essential.

Always bearing in mind that energy sustainability, and therefore environmental sustainability, is based on the search for new energy sources that reduce the abusive use of fossil fuels, that are cheaper and that their $\mathrm{CO}_{2}$ emissions are not so high. During the last decades there have arisen alternative mixtures to natural gas as it is the case of biogas $[6,7]$. This gas composed mainly of methane and carbon dioxide in variable proportions, depending on the organic matter from which it has been generated, is obtained from anaerobic fermentation, a microbiological bacterial process in the absence of oxygen, from organic matter in biogas digesters, with high variability in its composition.

The resulting biogas coming out of the digesters contains in addition to the major compounds $\mathrm{CH}_{4}((50$ a 60$) \%)$ y $\mathrm{CO}_{2}$ ((40 a 50$\left.) \%\right)$, contains other gases such as $\mathrm{N} 2, \mathrm{CO}, \mathrm{O}_{2}, \mathrm{H}_{2}, \mathrm{H}_{2} \mathrm{O}$ or $\mathrm{NH}_{3}$ in addition to other more harmful impurities such as $\mathrm{H} 2 \mathrm{~S}$ or siloxanes, that is why, that before its integration into natural gas 
distribution networks, raw biogas needs to be converted into biomethane by means of different purification or upgrading methods which range from $\mathrm{CO}_{2}$ removal by washing with water or organic solvents, activated carbon or membrane separation. The resulting biomethane will have a final composition of $\mathrm{CH}_{4}>95 \%$, $\mathrm{CO}_{2}<2 \% \mathrm{H}_{2} \mathrm{~S}<2 \mathrm{mg} \cdot \mathrm{m}^{-3}$ and siloxanes lower than $10 \mathrm{mg} \cdot \mathrm{m}^{-3}$.[8].

In European Union in 2016 the generation of biogas reached 16.1 Mtoe (ton equivalent of petroleum), with an electrical production from biogas of $62.5 \mathrm{TW}-\mathrm{h}$. The primary producer in biogas is so far is Germany with a total production of 7956.3 Mtoe, Spain is in tenth position with a production of 230.8 Mtoe [9]. In order to be able to contribute to the implementation of biogas in Spain in the distribution lines, it is necessary that they comply with certain specifications in the current regulation. It is for this reason that the knowledge of their thermodynamic properties is necessary in order to provide reliable data to improve the thermodynamic models used to calculate and design the extraction, transport, storage, distribution and quality standards of biogas mixtures.

\subsection{State of the art}

microwave-based devices have proven their applicability in the field of research into a wide range of thermophysical properties of pure substances and mixtures. In addition, microwave resonators have been widely characterized in order to investigate the phase equilibrium of pure fluids and the behavior $(p, T, x)$ of fluid mixtures, and this is the main objective of this doctoral thesis, which is to propose a new microwave resonator, with a cylindrical geometry and a tube inside the cell, which will contain the sample, in order to obtain accurate experimental data of quality and precision. 
Microwave techniques were first introduced by Rogers et al. [10]. Their equipment was designed to study the phase transition of corrosive substances at temperatures above $588 \mathrm{~K}$ and pressures up to $170 \mathrm{MPa}$. The evanescent system consisted of a cylindrical resonator operating at frequencies from 8 to $12 \mathrm{GHz}$, which was connected to the cell containing the high-pressure sample. The cavity and sample were separated by a sapphire window. The evanescent system had a total volume of $40 \mathrm{~cm}^{3}$, where the volume of the cell containing the sample occupied approximately $20 \mathrm{~cm}^{3}$. The construction allowed microwave penetration through the sapphire window to the fluid under study. The phase transition was determined by the change in resonance frequency and quality factor $\mathrm{Q}$. Rogers et al. [10] measured under isothermal conditions the dew and bubble points of pure substances such as propane or carbon dioxide and of systems containing oils with a resolution greater than $0.1 \mathrm{~K}$ and $0.07 \mathrm{MPa}$.

Fogh et al [11] presented an automatic system with variable volume. The equipment allowed measurements of dew and bubble points at temperatures between (253 to 473) K and pressures between (5 to 10) MPa. The cell containing the sample had an approximate volume of $10 \mathrm{~cm}^{3}$ and the phase envelopes of ethane + octane systems were measured. Using this same cell by Frørup et al. [12] who determined the dew and bubble points around the critical zone of ethane + heptane and ethane + octane systems. Later, Goodwin et al [13] used this same apparatus for the determination in isochoric conditions of dew points of methane + propane systems and, gravimetrically, prepared mixtures of different hydrocarbons which contained methane and icosane but from these systems there is no information concerning the uncertainties in the measurements of the published dew points. Subsequently using a reentrant cavity designed by Goodwin et al. [14] used at approximate frequencies at $375 \mathrm{MHz}$ determining phase envelopes near the critical zone in mixtures of carbon dioxide and methane at temperatures near $290 \mathrm{~K}$ and pressures in the range of 5 to $7 \mathrm{MPa}$. 
Currently using a reentrant cavity designed by Goodwin et al. [14] working at frequencies approximately $375 \mathrm{MHz}$ in order to detect phase envelopes near the critical zone, mixtures of carbon dioxide and methane at temperatures close to 290 $\mathrm{K}$ and pressures in the range of 5 to $7 \mathrm{MPa}$ were prepared.

The starting point for this doctoral thesis was the doctoral thesis work of Gómez [15], where a first prototype of the measuring cell used for the determination of phase envelopes at high pressure is described. With this first prototype it has been possible to determine the phase envelope of methane + carbon dioxide systems, and it has been possible to determine the phase envelopes of a synthetic biogas and a raw biogas obtained directly from the biomethanization plant of "La Paloma".

\subsection{Thesis doctoral aims}

The main objectives of this doctoral thesis are:

-To design a new cylindrical cavity based on the proposal by Gómez [15] with which to carry out experimental measures in the equilibrium of phases at high pressures without the inconveniences that the previous team had.

-Validate this technique with a substance whose thermodynamic properties (carbon dioxide) are well known and compare the results of deviations obtained experimentally with the equations of state, Span \& Wagner [16].

-Knowledge of the phase envelope of the binary mixtures formed by $\left(\mathrm{CO}_{2}+\mathrm{CH}_{4}\right),\left(\mathrm{CO}_{2}+\mathrm{C}_{3} \mathrm{H}_{8}\right)$ y $\left(\mathrm{CO}_{2}+\mathrm{N}_{2}\right)$ at different compositions in molar fraction of $\mathrm{CO} 2$ in the temperature range of $229.15 \mathrm{~K}$ up to the point of maximum temperature (cricondentherm).

-Elaborate the calculation procedure for the treatment of experimental data and the analysis of uncertainties following the state of the art bibliography. 
- Validation of the equations of state used in the gas industry by means of the GERG 2008 equation [4] and by means of some of the most used cubic equations of state in thermodynamic treatment such as those of PengRobinson [17] using the Wong-Sandler mixing rules [18], by means of the comparative study of the relative deviations with respect to the experimental results.

- Contribution to the thermodynamic database with new quality and accurate experimental data for the better development of the models used.

\subsection{Structure of the thesis}

The general structure of this doctoral thesis is as follows:

Chapter 1 presents a brief introduction of the motivations that give rise to the development of the work, describes the objectives that are intended to address in the study of mixtures and puts into context the background on which this experimental technique is based.

Chapter 2 describes the thermodynamic properties of fluids, on high-pressure phase equilibrium is of the types of diagrams that the experimental data return in functions of the variables that are represented. In addition to a study of the electromagnetic properties of fluids, which describes the concepts of dielectric constant and microwave network, on which the experimental technique developed is based.

Chapter 3 details a bibliographic review of the experimental techniques on which the experimental equipment presented in this doctoral thesis is based. This chapter also describes the basic principle on which the measuring cell is based and the peculiar characteristics that make this equipment a novel technique.

Chapter 4 presents the experimental equipment where a description of the technique presented is made, the parts of which the equipment is composed are 
detailed, especially the measuring cell, and finally, the software used for the acquisition of experimental data is detailed.

Chapter 5 presents the results obtained when validating the experimental technique with $\mathrm{CO}_{2}$ describing the procedure established for the acquisition of experimental data, and details the procedure for calculating uncertainties using the guide for the expression of measurement uncertainty (JCGM 106 2012) [19]. Finally, we present the experimental results obtained comparing these with the Span \& Wagner equation of state [16] implemented in the NIST REFPROP software [20], and with the literature found.

Chapter 6 shows the results obtained in the determination of the phase envelope of six binary mixtures formed by $\mathrm{CH}_{4}, \mathrm{C}_{3} \mathrm{H}_{8}$ and $\mathrm{N}_{2}$ to different $\mathrm{CO}_{2}$ compositions. All these mixtures were been studied in the range of temperature within the limits of the thermostatic bath that establish as lower limit $233.15 \mathrm{~K}$ reaching the maximum temperature of each one systems (cricondentherm), the experimental measures were also been carried out in the proximities to the critical point and in the retrograde condensation zone. The chapter also describes the way in which the samples were prepared and the procedure carried out to obtain the experimental data. A study of uncertainty is also carried out using the guide for the expression of measurement uncertainty" (JCGM 106 2012) [19]. Experimental data were comparing with the GERG-2008 equation of state [4] implemented in the NIST REFPROP database [20], the experimental data obtained and were been correlated with the dielectric properties using the procedure established by Harvey and Lemmon [21] and the modeling results are presented using the Peng-Robinson equation of state [17] using the Wong-Sandler mixing rules [18].

Chapter 7 lists the conclusions and contributions of this work.

Chapter 8 presents a brief summary in English in order to meet the requirements of a doctoral thesis paper with an international mention. 

CHAPTER 2

\section{Thermophysical properties}





\section{Chapter 2. Thermophysical properties}

\subsection{Introduction}

The thermodynamic state is determined by its pressure, volume and temperature. In general, these quantities cannot vary all of them independently. In mathematical terms, there is a relationship between these quantities, which can be symbolically represented as $\mathrm{V}=\mathrm{f}(\mathrm{p}, \mathrm{T}, \mathrm{m})$. In more general problems other state variables are needed, such as magnetization or electrical polarization. In this doctoral thesis we will focus on the properties of phase equilibrium and electromagnetic properties, since the experimental technique developed is based on the latter. Within the electromagnetic properties, main attention will be paid to the properties of dielectrics, this is due to the fact that the magnetic susceptibility of the substances studied in this doctoral thesis can be considered negligible compared to dielectric permittivity.

The relationship between the volumetric properties in their different phases and the dielectric properties, will be studied in this doctoral thesis and how they can be related to the resonance frequency. In this chapter, we will present the theoretical framework related to the properties of equilibrium and which are the equations that govern its behavior. In addition, we will study the properties of dielectrics and their relationship with fluids, studying their polarization phenomena. Finally, a study will be made of the existing relationship between volumetric and dielectric properties and how these are correlated with changes in frequency. 


\subsection{Equilibrium properties}

In thermodynamics, where attention is always focused on a certain amount of matter, a situation of equilibrium means that there is no change in the properties of the material over time. In reality, a true state of equilibrium is probably never reached due to continuous variations in the surroundings and inertia in possible changes.

Equilibrium requires a balance of all the potentials that can cause a change; however, the speed of change and, therefore, the speed of approach to equilibrium is proportional to the difference in potential between the actual state and the state of equilibrium $[22,23]$.

\subsection{The chemical potential}

The macroscopic properties of homogeneous fluids that are in equilibrium are functions of pressure, temperature and composition. In a multicomponent system and taking into account the number of moles of one of the components, by varying a thermodynamic property such as, for example, the Gibbs energy, an extensive magnitude of great importance for the study of thermodynamic equilibrium, remaining constant, pressure, temperature and the number of moles of the rest of the components arises, the definition of a new extensive property called chemical potential $\left(\mu_{i}\right)$. A very important equation in the phase equilibrium theory is the Gibbs-Duhem equation [24].

$$
d G=V d p-S d T+\sum_{i=1} \mu_{i} n_{i}
$$


Gibbs-Duhem equation 2.1 is a fundamental equation in solution thermodynamics.

\subsection{Fugacity coefficient}

Gibbs energy is a very important thermodynamic property given its strong relationship with temperature and pressure through the equation.

$$
\mathrm{dG}=-\mathrm{SdT}+\mathrm{Vdp}
$$

the simplicity of this equation requires the need to replace pressure with a new property, which G.N. Lewis [25] defined as fugacity (f). The reason for this definition is that fugacity for a non-ideal system should be identified with pressure, so equation 2.2 can be written.

$$
\left(\mathrm{dG}_{\mathrm{i}}\right)_{\mathrm{T}}=\mathrm{RTd}\left(\operatorname{lnp}_{\mathrm{i}}\right)
$$

where $a f_{i}$, is the fugacity of the pure component $i$. When equation 2.3 is applied to a mixture, as is the case of this doctoral thesis the above equation can be rewritten as follows.

$$
\mathrm{d} \mu_{\mathrm{i}}=\operatorname{RTd}\left(\ln \hat{f}_{\mathrm{i}}\right)
$$

The importance of the concept of fugacity is reflected in the fact that it can be used as a phase equilibrium criterion, in a similar way as for chemical potentials. That is to say, the fugacity $f_{i}$ of any component in a multicomponent system must be the same in all the phases in which it is present, for the system to be in equilibrium. 


$$
\mathrm{f}_{\mathrm{i}}^{1}=\mathrm{f}_{\mathrm{i}}^{2}=\cdots \mathrm{f}_{\mathrm{i}}^{\pi}
$$

\subsection{Electromagnetic properties of fluids}

Electromagnetic fluids, are studied at microscopic and macroscopic scales. We refer to microscopic scale to the energy bands and the magnetic moment of the atoms and molecules in them, while, in the macroscopic scale, we study the general response to electromagnetic fields [31]. At macroscopic scale the interactions between fluid and electromagnetic fields are defined by Maxwell's equations.

The fluids under study will be considered as dielectric materials, i.e. they will have zero conductivity and their magnetic permeability is similar to that of vacuum. Another important consideration is that the applied electromagnetic fields are weak and very far from the values that produce the rupture of the dielectric and the ionization of the molecules. When an electric field is applied to a fluid in its pure state, its response can be classified into two categories; polar and non-polar fluids [32].

Polar fluids are those whose molecules have a permanent dipolar moment. They have to be compared with non-polar fluids, which their molecules only have an electrical polarization network when an external electric field is applied. Polar fluids have a high relative permittivity with values in the range of $10-100$. This causes dipoles in the permanent molecules. Polar fluids also have high dielectric losses in microwave frequencies, with tangential dielectric losses in the range of 0.1-1, derived from the relaxation of molecular orientations when altered electric fields are applied. 


\subsection{Dielectric constant}

When dielectric fluids are subjected to high frequency electromagnetic fields, for example (as in this doctoral thesis), microwaves, the permanent dipoles of the molecules cannot follow the variation of the electric field, lose the static dielectric constant and cause a dissipation of energy [31].

Most pure fluids and their mixtures can be considered to behave as dielectric materials as a function of their electromagnetic properties. A dielectric fluid inserted in a capacitor provides the equivalent circuit defined in figure 2.2 (Spanish version, page 22).

Figure 2.3 (Spanish version, page 25) shows qualitatively the behaviour of permittivity $\left(\varepsilon_{\mathrm{r}}^{\prime} \mathrm{y} \varepsilon_{\mathrm{r}}^{\prime \prime}\right)$ as a function of frequency [34]. From the microscopic point of view permittivity is related to several physical phenomena: Ionic conduction, dipolar relaxation, atomic polarization and electronic polarization are the mechanisms that contribute to the permittivity of a dielectric fluid. In the low frequency range, $\varepsilon^{\prime \prime}$ is influenced by ionic conductivity [31]. The variation in permittivity in the microwave range is mainly caused by dipolar relaxation, and the absorption peaks in the infrared and upper region are mainly due to electronic and atomic polarization.

\subsection{Microwave network}

The microwave network concept is developed from transmission line theory, and is a powerful tool in microwave engineering. The microwave network method studies the response of a microwave structure, in our case the resonant cavity, to external signals and is a complement to the field theory that analyzes the field distribution within the microwave structure [31]. 
The behavior of radiofrequency devices is characterized by the use of dispersion parameters $S$ which are relations of magnitude and phase of the incident and reflected power waves found in the ports of entry and exit. A very convenient analogy to explain these power relations is by means of an optical simile [35] as can be observed in figure 2.4 (Spanish version, page 27).

One of the fundamental concepts of microwave networks is related to incident waves, transmitted and reflected along transmission lines. To understand this concept is helpful the analogy of a light wave that passes through an optical lens, part of the light is reflected by the lens surface, but most of the light passes through the lens. Microwave network analysis involves of accurately measuring the relationships between the incident signal, the transmitted signal, and the reflected signal.

Figure 2.5 (Spanish version, page 28) shows the block circuit of the microwave network composed of the resonant cavity and its couplings, the microwave input to ports 1 and 2 are denoted as $a_{1}$ and $a_{2}$ respectively, and the microwave output to ports 1 and 2 are denoted $a s b_{1}$ and $b_{2}$ respectively.

\subsection{Relationship between volumetric and dielectric properties}

Relative permittivity is high if the molecules are polar or highly polarizable, the quantitative relationship between relative permittivity and the dielectric properties of the molecules is expressed by Debye's equation.

$$
\frac{\varepsilon_{\mathrm{r}}-1}{\varepsilon_{\mathrm{r}}+2}=\frac{\rho \mathrm{P}_{\mathrm{m}}}{\mathrm{M}}
$$

When a phase change occurs, either bubble point or dew point, this effect results in an alteration in the volumetric properties of the substance, this alteration 
is related to the dielectric constant of the material containing the sample, this relationship between the volumetric properties and the dielectric constant can be expressed by Clausius Mossotti equation for non-polar fluids.

$$
\frac{\varepsilon_{\mathrm{r}}-1}{\varepsilon_{\mathrm{r}}+2}=\frac{\rho \mathrm{N}_{\mathrm{A}} \alpha}{3 \mathrm{M} \varepsilon_{0}}
$$

the discontinuity caused by the variation in density at the phase change, becomes a discontinuity in the dielectric constant The Clausius-Mossotti equation applies when the dipolar momentum does not contribute to polarization [36], which occurs when the molecules are non-polar or because the frequency of the external field is so high that the molecules cannot be oriented in the direction of the field. 

CHAPTER 3

Experimental technique for high pressure equilibria determination 



\section{Chapter 3. Experimental techniques for high pressure equilibria determination}

\subsection{Introduction}

When a new technique is developed or an improvement is made in an already established one, such as in the case of this doctoral thesis, it is necessary to carry out a bibliographic review of the existing experimental techniques in order to be able to contrast it's potential contributions. It is also necessary to know the principle of operation on which it is based and its physical and mathematical basis. In this chapter, we will try to answer these questions, the first part focuses on a general review of the high-pressure experimental techniques that are currently being used to measure the equilibrium of phases. The second part of this chapter is devoted to a more focused review of the techniques on which this research work is based, as well as the principle that governs it and the equations that solve it and give meaning to the experimental data obtained.

\subsection{Experimental techniques for high pressure equilibrium measurement}

The knowledge of high pressure phase equilibrium is important not only for the design, optimization of chemical processes and separation operations, also necessary for the simulation of oil reserves, capture and storage of $\mathrm{CO}_{2}$, transport and storage of natural gas, refrigeration cycles and heat pumps, and the study of geological processes. As well as in the new green chemistry, that uses supercritical $\mathrm{CO}_{2}$ and has many industrial applications [27]. Similarly, experimental data are necessary for the processing, enrichment and storage of biogas application of which is the objective this work. On the other hand, the estimation of equilibrium data at high pressures is, in many cases, difficult and complex because the 
deviations from the ideality are much greater. So that, obtaining experimental data is often the only appropriate method to obtain reliable data, as unanimously recognized by the scientific community and industry, in certain industrial applications.

Experimental data are also very important for validating thermodynamic property estimation models.

It is virtually impossible to obtain high-pressure experimental data from all kinds of systems using a single technique. The reason for this is that experiments have to be performed in a wide range of pressures and temperatures, and for mixtures with components with different physical properties. Accurate experimental determination of phase equilibrium data is most often difficult and costly in time and money, but on the other hand, the application of inaccurate estimated data can be much costlier in certain cases. Several authors since 1986 have conducted reviews and studies of published experimental methods $[37,38,39,40,41]$. Different methods have been used to obtain experimental data on high-pressure phase equilibrium; each method has application in different experimental conditions. In the period 2005-2008 Fonseca et al. [27] classify the experimental methods into two large groups depending on how the phase compositions are determined: analytical methods and synthetic methods. They study a total of 791 scientific articles from experimental data of high-pressure phase equilibrium. Fonseca et al. [39] draw up a classification diagram of the experimental methods shown in the figure 3.1 (Spanish version, page 37 ). 


\subsection{Experimental techniques for the measurement of dielectric properties}

Measurements of relative electrical permittivity or dielectric constant, such as a function of pressure or temperature $\varepsilon(T, P)$, can be used to determine a wide range of thermodynamic properties [40]. Measurements of the dielectric and magnetic constant based on radio or microwave methods. As occupied by this thesis, have been studied in depth. Afsar et al. [43] covered most of the techniques used to measure the properties of materials in the frequency from $1 \mathrm{MHz}$ to 1500 $\mathrm{MHz}$. In this case, the use of a sample tube (quartz) in a resonant cavity was proposed by Asmussen et al. [44] for plasma induction. On the other hand, Gregory y Clark [32] have carried out an extensive review of the different methods for the measurement of the dielectric constant of polar liquids. Some of the techniques on which are based the experimental equipment presented in this thesis will be named in this section.

The method of perturbation in the use of cylindrical cavities is one of the most commonly used techniques. Doing a wide bibliographic review, we can find a temporal regime in the evolution of this experimental technique and as various authors have modified in various ways a technique that is still in its infancy.

Starting with Ewing \& Royal [55], they built a resonator (using brass as material), consisting of an open-ended cylindrical section with a volume of $5.7 \mathrm{~cm}^{3}$ and upper and lower plates that closed the cavity as shown in figure 3.8 (Spanish version, page 45). Using the non-degenerated modes TM010, TM011 and the doubledegenerated mode TM110, they developed a theoretical model with which they determined the relative permittivity of nitrogen at $300 \mathrm{~K}$.

Kapilevich et al. [56,57] used a silver cylindrical cavity, figure 3.9 (Spanish version, page 46), with a sample tube in its central axis of different diameters ranging from 0.25 to $0.3 \mathrm{~mm}$ for the outer diameter and 0.8 to $0.9 \mathrm{~mm}$ for the inner 
diameter, and operating with a resonance mode TM101 at $3 \mathrm{GHz}$ determined the value of the dielectric constant of various biological fluids.

More recently Kanpan et al. [62] developed a cylindrical cavity, figure 3.11 (Spanish version, page 47), built in aluminum and with a hole in its central axis where was housed a PTFE tube of $0.12 \mathrm{~cm}^{3}$ of volume to contain the sample. they measured the resonance mode TM010 to $3 \mathrm{GHz}$ and used the method of perturbations and an analysis based on method of Ritz-Galerkin, to study the permittivity of fluids such as ethanol, methanol and distilled water, observing that in a precise way with small quantities of samples determined.

\subsection{Cylindrical resonant cavity in microwave basic principle}

When a phase transition is originated, there is a change in density with respect to the homogeneous phase, this causes a discontinuity in the dielectric constant of the new phase. The relationship between density and dielectric constant is determined as discussed above by the Clausius-Mossotti (equation 2.7). In order to demonstrate the principle, the methods of perturbations in resonant cavities are used for the study of electromagnetic properties of materials. Birnbaum y Franeau [64] first developed the resonant cavity perturbation method in 1949 for the measurement of the dielectric constant in solids and liquids. An electromagnetic system, such us, a resonant cavity in microwaves with a sapphire tube in its central axis, which contains the sample to study, can be defined by a series of parameters as they are: the dimensions of the resonator, the conductivity of its walls, the dielectric permittivity and the magnetic permeability of the medium occupied in the space of the cavity.

The dielectric permittivity and magnetic permeability are calculated from the changes of the resonance frequency and the quality factor of the cavity in the vacuum, and when it contains the sample object of measurement. The phase 
transitions studied in this doctoral thesis (bubble point and dew point) can be considered as disturbances of the dielectric medium, since the dielectric permittivity of the liquid or vapor phase can differ significantly.

Considering a resonant cavity constructed with an ideal conductive material [31], and enclosed in a known surface $S$ with a constant volume $V$, the electric and magnetic fields before the disturbance are $E_{1}$ and $H_{1}$. According to Maxwell's equations (equations 3.3 and 3.4, Spanish version, page 50). 

CHAPTER 4

\section{Experimental setup}





\section{Chapter 4. Experimental setup}

\subsection{Introduction}

Until now, the previous chapters have developed the theoretical framework of resonant cavities in microwaves by means of the perturbation method, with a detailed bibliographic review of the experimental techniques for the measurement of the fluid dielectric constant. The following chapter will describe in detail the technical and constructive aspects of the measuring cell, which together with the vector network analyzer (VNA) are the most important parts for the development of this research work. In addition, the technical aspects of the auxiliary devices of which the experimental equipment is composed and the software used for the automatic control and data acquisition of all the elements of the equipment will be detailed.

The apparatus described in this section consists of an original work designed in the TERMOCAL (thermodynamic and calibration laboratory of the University of Valladolid), and built by DG-Technology.

\subsection{Cylindrical cavity resonator description}

In general, a microwave cavity is a metal enclosure, which allows an electromagnetic oscillation formed by the short circuit of two ends of a waveguide. The electromagnetic energy is stored within the cavity enclosure and the energy is dissipated in the metal wall and the dielectric material within the cavity [59]. As the electrical and magnetic waves reflect off the axial limits, patterns of stationary waves form the resonant modes of the cavity. The modes are defined as a pattern of movement that is repeated sinusoidally [69]. In order to achieve coupling of the 
cavity to an external source for the purpose of excitation of a particular mode, a probe or test tube may be used.

The design of the cavity is based on the concept described by Gómez [15] which has had a series of improvements in both design and functionality. Figure 4.1 (Spanish version, page 73) shows a section view of the cylindrical cavity used in this work, the main cavity is constructed in a 15\% copper-zirconium alloy, (ZrK015) supplied by Luvata ${ }^{\circledR}$, in order to prevent possible corrosion on copper cavities, a finish, on the inside face, of "mirror" cavity provides an improvement in the quality factor of the resonant modes. The cavity has an inside diameter of $50 \mathrm{~mm}$ and a height of $50 \mathrm{~mm}$, with a total volume of approximately $98 \mathrm{~cm}^{3}$. The cavity is delimited by three parts, the main cylindrical body, and two lids which are fitted to the main body by means of eight M6 threaded rods which completely enclose the cavity, and a Kalrez ${ }^{\circledR}$ O-ring which provides a hermetic seal in the cavity. Cylindrical pins Goupilles Clindriques ISO2338 have been fitted to ensure a good fit between the covers and the cavity.

To perform the vacuum inside the cavity, in the lower cover, there is a hole from which a rotary vacuum pump type Oerlikon TRIVAC D8B is coupled. The center of the cavity is drilled in such a way that the sapphire tube containing the sample can be housed in it, and whose clamping system will be discussed later, these holes are provided with a recess that allows an O-ring of the commercial brand described above to be housed and allows the vacuum to be contained inside the cavity.

\subsection{Experimental setup}

The measuring cell is immersed in a Fluke model 7037 thermostatic bath that allows working in a temperature range from (233.15 to 423.15$) \mathrm{K}$ with a homogeneity of $\pm 0.01{ }^{\circ} \mathrm{C}$. The fluid in which the measuring cell is immersed is ethanol in order to achieve temperatures below $0{ }^{\circ} \mathrm{C}$. Figure 4.4 shows a diagram 
of the experimental equipment, the measuring principle, as well as the most important parts are shown in this section.

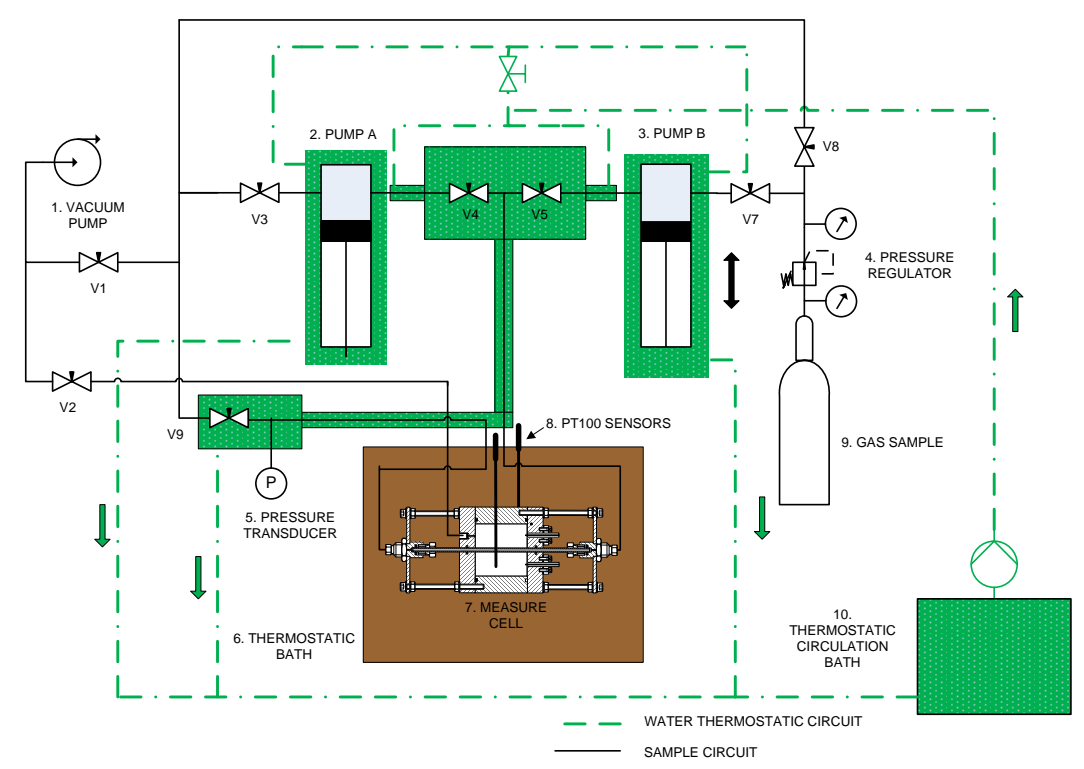

Figure 4.1 Experimental setup and auxiliary devices employed in this $\mathrm{PhD}$.

Figure 4.2 shows a photograph of the experimental equipment used in this work, this is located in an aluminum structure divided into two levels, in the lower level are located the gaseous samples for study, the vacuum pump and the bath for the thermostatic circuit. In the upper level, there are the devices for measuring temperature, pressure and the thermostatic bath in which the measuring cell is immersed. 

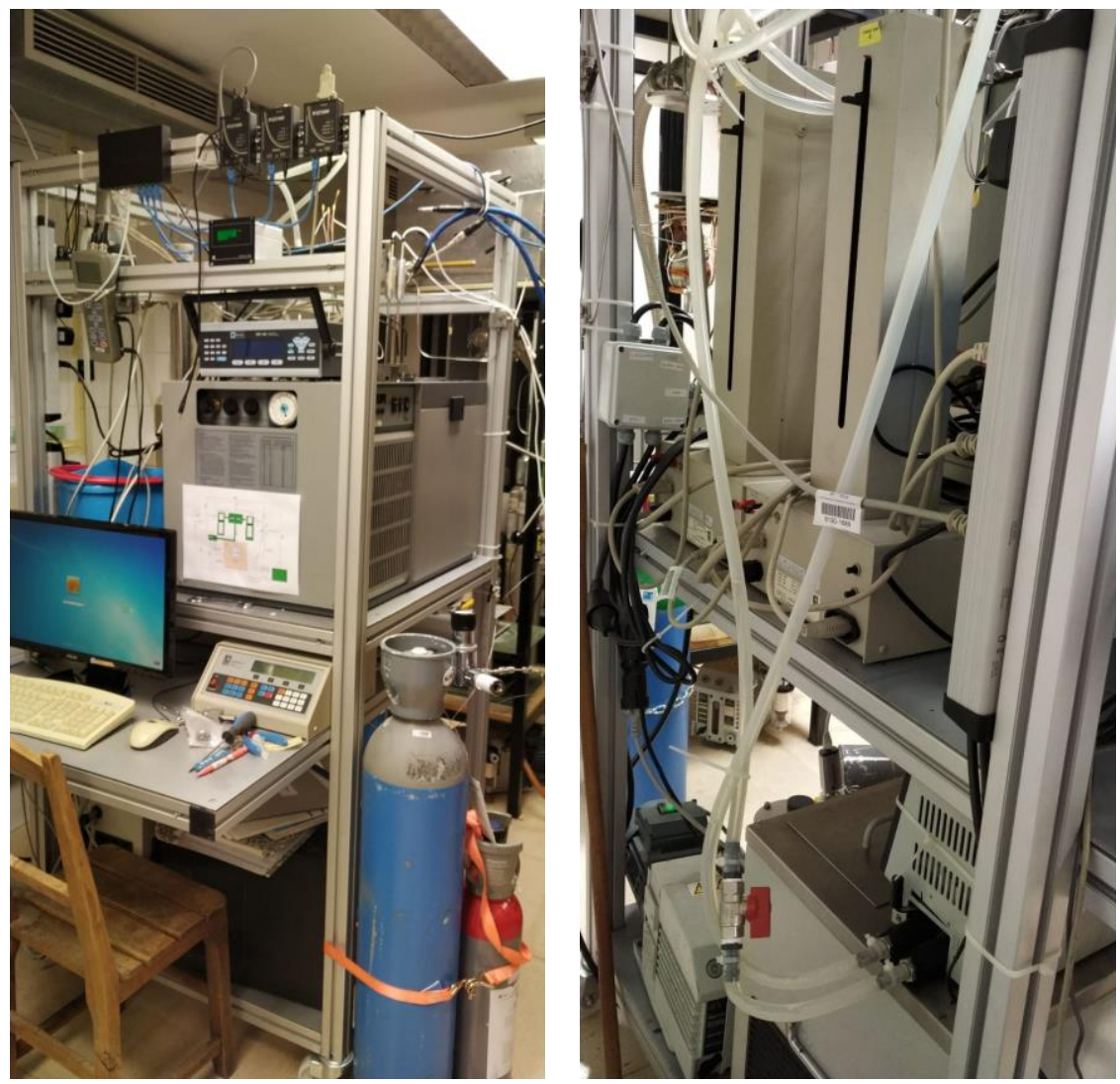

Figure 4.2 Frontal view (left) and back view (right) of the experimental setup.

\subsection{Control software}

The acquisition of experimental data is a complex task if the necessary equipment is not available for this purpose all the auxiliary devices of the experimental technique have been connected through a computer that will allow us to store and manage all the experimental data that are necessary to obtain the desired information. Given that not all the measuring instruments have the same connections, the computer has chosen to unify all of them using RJ45 Ethernet ports. Those elements with RS232 connections such as the pressure sensor, the ISCO pumps and the thermostatic bath, were connected to Ethernet ports by 
means of external devices of the commercial brand WIZnet ${ }^{\mathrm{TM}}$ model WIZ100 ${ }^{\mathrm{TM}}$, which allow transforming the RS232 port input connection and an RJ45 port as an exit connection. The control software, that provides the same commercial brand was able to configure the technical aspects of these equipment so that they are recognized as network elements and therefore controlled by the computer. With the exception of the temperature reader that is connected by means of USB, the rest of the devices are interconnected by means of an 8-port Switch of the commercial brand D-Link ${ }^{\mathrm{TM}}$. With all the auxiliary elements connected in network, by means of the remote desktop we will be able and control accede to the experimental equipment from any computer and to be able to control.

The software used to manage the control of the experimental equipment was the VEE Pro $9.3^{\circledR}$ supplied by Keysight Technologies, starting from the base program that managed the VNA developed by Gómez [15], the rest of programs capable of controlling the most important auxiliary devices for the measurement of the phase equilibria have been programmed. Figures 4.6 to 4.8 (Spanish version $82,83)$ show the control panels of the programs created for control and data acquisition. 

CHAPTER 5

\section{$\mathrm{CO}_{2}$ measurements}





\section{Chapter $5 . \quad \mathrm{CO}_{2}$ measurements}

\subsection{Introduction}

When a new experimental technique is developed either by means of a series of modifications that in some way improve this technique, or by the design of a new experimental technique, a validation procedure is necessary to give truthfulness to the experimental data that are intended to be obtained. In order to achieve this, a reference fluid is used whose thermodynamic properties are well known, so that, they can be compared with the results obtained with the new technique. Carbon dioxide has been the substance used, for the validation of the technique as it is a substance whose physical and thermodynamic property are well known. This is a substance that does not present permanent dipolar moment, although it does quadrupole and therefore there is electrostatic interaction between its molecules, reason why it can be considered a non-polar substance.

This section will focus on the determination of the vaporization curve (dew and bubble points) comparing the experimental data with the equation of state of Span \& Wagner [16] implemented in the database in the NIST REFPROP [20], in addition the obtained experimental data have been correlated with the dielectric properties using the procedure established by Harvey \& Lemmon [21], the resonance frequencies obtained in each of the tests will validate the theory of perturbations described in section 3.4 of this work.

\subsection{Experimental procedure}

Using a carbon dioxide supplied by air liquid with a purity of $99.999 \%$, this section describes the experimental procedure performed to determine the vaporization curve. Experimental data were determined with respect to the 
pressure along an isotherm, the isothermal variation was carried out using the Fluke bath model 7037. The estimated time to guarantee the correct temperature in the resonator was 2 hours after the bath reached the working temperature. Once the bottle has been connected to the main line, the vacuum was made in the whole circuit including the cavity and the sapphire tube that will contain the sample. This process will continue for 1 hour in order to ensure that possible impurities are dragged both in the line and in the cavity.

After this period of time, the ISCO 260D syringe pumps are filled with $\mathrm{CO}_{2}$, which will be responsible for the positive (determination of the dew point) and negative (determination of the bubble point) displacements. The starting pressure, regardless of the phase change to be determined, will be $20 \%$ lower or higher than the estimated value to ensure that the phase at which the sample is found is gaseous, if you want to determine the dew point, or liquid if you want to determine the bubble point. The flow rate provided by the pumps will be sufficient to observe a quantifiable change at the time of the phase change, this value has been set at $1.00 \mathrm{ml} / \mathrm{min}$ for both experiments.

Always starting from the gaseous phase and under the conditions described above, the experiment begins with positive displacement of the pumps until reaching the phase change, (dew point). After 1 hour of waiting to ensure homogeneity, the negative displacement is carried out until the phase change is again determined (bubble point). Once the experimental data are obtained, the pumps will be returned to a state that allows new points to be determined under new temperature conditions.

\subsection{Uncertainty Determination}

The combined expanded uncertainty of bubble and dew point measurements under pressure and temperature conditions has been determined according to the 
"Guide to the Expression of Uncertainty Measurement “ (JCGM 106 2012) [19]. The measurement of the expanded uncertainty for temperature $U(T)$ in the measurement of bubble and dew points can be estimated by:

$$
\mathrm{U}(\mathrm{T})=2 \cdot\left[\mathrm{u}\left(\mathrm{T}_{\text {res }}\right)^{2}+\mathrm{u}\left(\mathrm{T}_{\text {rep }}\right)^{2}+\mathrm{u}\left(\mathrm{T}_{\mathrm{cal}}\right)^{2}\right]^{1 / 2}
$$

Where $\mathrm{u}\left(\mathrm{T}_{\text {res }}\right)$ represents the standard uncertainty due to temperature device resolution, an ASL F100 device is in charge of obtaining temperature probe readings, this device has a resolution of $\mathrm{u}\left(\mathrm{T}_{\text {res }}\right)=0.001 \mathrm{~K}$. For $\mathrm{u}\left(\mathrm{T}_{\text {rep }}\right)$ it refers to the standard uncertainty due to the reproducibility of the temperature measurement under the conditions in which the phase change originates (dew or bubble). Finally, the term $\mathrm{u}\left(\mathrm{T}_{\text {cal }}\right)$, is established as the standard uncertainty due to the calibration of the temperature probes, this uncertainty is established in $\mathrm{u}\left(\mathrm{T}_{\text {cal }}\right)=0.015 \mathrm{~K}$.

When determining the expanded uncertainty $U\left(T_{C}\right)$ at the critical point it is necessary to introduce a new contribution to equation $5.1 \mathrm{u}\left(\mathrm{T}_{\text {interp }}\right)$, due to the adjustment polynomial that was used in order to determine, by interpolation of the experimental data, the critical point, leaving the previous equation as follows.

$$
\mathrm{U}\left(\mathrm{T}_{\mathrm{C}}\right)=2 \cdot\left[\mathrm{u}\left(\mathrm{T}_{\text {res }}\right)^{2}+\mathrm{u}\left(\mathrm{T}_{\text {rep }}\right)^{2}+\mathrm{u}\left(\mathrm{T}_{\text {cal }}\right)^{2}+\mathrm{u}\left(\mathrm{T}_{\text {interp }}\right)^{2}\right]^{1 / 2}
$$

The expanded uncertainty in the measurement of pressure $U(p)$ analogously to the expanded uncertainty due to temperature can be determined from the expression:

$$
\mathrm{U}(\mathrm{p})=2 \cdot\left[\mathrm{u}\left(\mathrm{p}_{\text {res }}\right)^{2}+\mathrm{u}\left(\mathrm{p}_{\text {rep }}\right)^{2}+\mathrm{u}\left(\mathrm{p}_{\text {cal }}\right)^{2}+\mathrm{u}\left(\mathrm{p}_{\text {lineal }}\right)^{2}\right]^{1 / 2}
$$


Thus, analogous to the calculation of the standard uncertainty of temperature, the uncertainty due to resolution is set at $\mathrm{u}\left(\mathrm{p}_{\mathrm{res}}\right)=0.001 \mathrm{MPa}$ which is the resolution of the multifunction indicator Druck DPI 145. The standard uncertainty due to repeatability $u\left(p_{\text {rep }}\right)$. Finally, the uncertainty obtained by calibration, was $\mathrm{u}\left(\mathrm{p}_{\text {cal }}\right)=3.75 \cdot 10^{-5}(\mathrm{p} / \mathrm{Pa})+100 \mathrm{~Pa}$. Due to the statistical method used for the determination of the points in the phase changes it is necessary to introduce a $\mathrm{u}\left(\mathrm{p}_{\text {lineal }}\right)$ contribution to the uncertainty due to the intersection of the lines.

Analogous to the determination of the expanded uncertainty of the critical temperature, the same interpolation polynomial is used for the determination of the critical pressure, so it is necessary to introduce in substitution of the contribution due to the intersection of the lines $\mathrm{u}\left(\mathrm{p}_{\text {lineal }}\right)$, a contribution $\mathrm{u}\left(\mathrm{p}_{\text {interp }}\right)$ due to the adjustment polynomial used in the determination, by interpolation of the experimental data of the critical pressure leaving equation 5.3 as follows:

$$
\mathrm{U}\left(\mathrm{p}_{\mathrm{C}}\right)=2 \cdot\left[\mathrm{u}\left(\mathrm{p}_{\text {res }}\right)^{2}+\mathrm{u}\left(\mathrm{p}_{\text {rep }}\right)^{2}+\mathrm{u}\left(\mathrm{p}_{\mathrm{cal}}\right)^{2}+\mathrm{u}\left(\mathrm{p}_{\text {interp }}\right)^{2}\right]^{1 / 2}
$$

\subsection{Experimental results}

Figures 5.1 and 5.2 (Spanish version 93,94) show two sample of the graphs obtained by measuring the phase change (bubble and dew point) carbon dioxide at $288.35 \mathrm{~K}$, where pressure a normalized frequency plotted as a function of the frequency. The phase change is characterized, first of all, by a change in the resonance frequency caused by a change in the dielectric constant of the substance, as well, as during the phase change, the pressure of the substance remains constant. 
Table 5.1 shows the experimental results of the bubble points, dew points and resonance frequencies of carbon dioxide and their comparison with the values returned by the equation of state Span \& Wagner [16].

Table 5.1: Experimental data of bubble, dew points and resonance frequency for carbon dioxide with expanded uncertainty $(k=2)$ in $U(T)=0.05 \mathrm{~K} ; \mathrm{U}(\mathrm{p})=0.010 \mathrm{MPa}$. Pressures calculated with Span \& Wagner [16] equation of state.

\begin{tabular}{l|rcccc} 
Set up & $\mathbf{T}_{\text {exp }} / \mathbf{K}$ & $\mathbf{p}_{\text {exp }} / \mathbf{M P a}$ & $\mathbf{f}_{\text {exp }} / \mathbf{G H z}$ & $\mathbf{p}_{\text {Eos }} / \mathbf{M P a}$ & $\delta \mathbf{p} \%$ \\
\hline Bubble & 273.35 & 3.502 & 4.1302 & 3.503 & -0.048 \\
Bubble & 278.36 & 3.989 & 4.1299 & 3.991 & -0.041 \\
Bubble & 283.35 & 4.521 & 4.1297 & 4.525 & -0.090 \\
Bubble & 288.35 & 5.107 & 4.1295 & 5.112 & -0.090 \\
Bubble & 293.36 & 5.753 & 4.1293 & 5.757 & -0.084 \\
Bubble & 298.35 & 6.461 & 4.1293 & 6.464 & -0.046 \\
Bubble & 300.35 & 6.762 & 4.1294 & 6.767 & -0.070 \\
Bubble & 302.35 & 7.076 & 4.1296 & 7.083 & -0.099 \\
Dew & 273.35 & 3.504 & 4.1330 & 3.503 & 0.015 \\
Dew & 278.36 & 3.992 & 4.1328 & 3.991 & 0.037 \\
Dew & 283.35 & 4.526 & 4.1324 & 4.525 & 0.030 \\
Dew & 288.35 & 5.115 & 4.1322 & 5.112 & 0.055 \\
Dew & 293.36 & 5.760 & 4.1320 & 5.757 & 0.039 \\
Dew & 298.35 & 6.462 & 4.1316 & 6.464 & -0.031 \\
Dew & 300.35 & 6.766 & 4.1313 & 6.767 & -0.017 \\
Dew & 302.35 & 7.077 & 4.1310 & 7.083 & -0.081 \\
Sp \% $\left(\frac{p_{\text {exp }}}{p_{\text {Eos }}}-1\right) * 100$ & & & &
\end{tabular}

Figure 5.1 shows the experimental data compared with the most relevant literature in the temperature range of $273.15 \mathrm{~K}$ to $304.00 \mathrm{~K}$ near the critical point, compared with the $\mathrm{CO}_{2}$ state equation developed by Span \& Wagner [16] implemented in REFPROP software [20] and compared with the literature by Kim et al. [72], Saleh et al. [73], and Gómez [15]. 


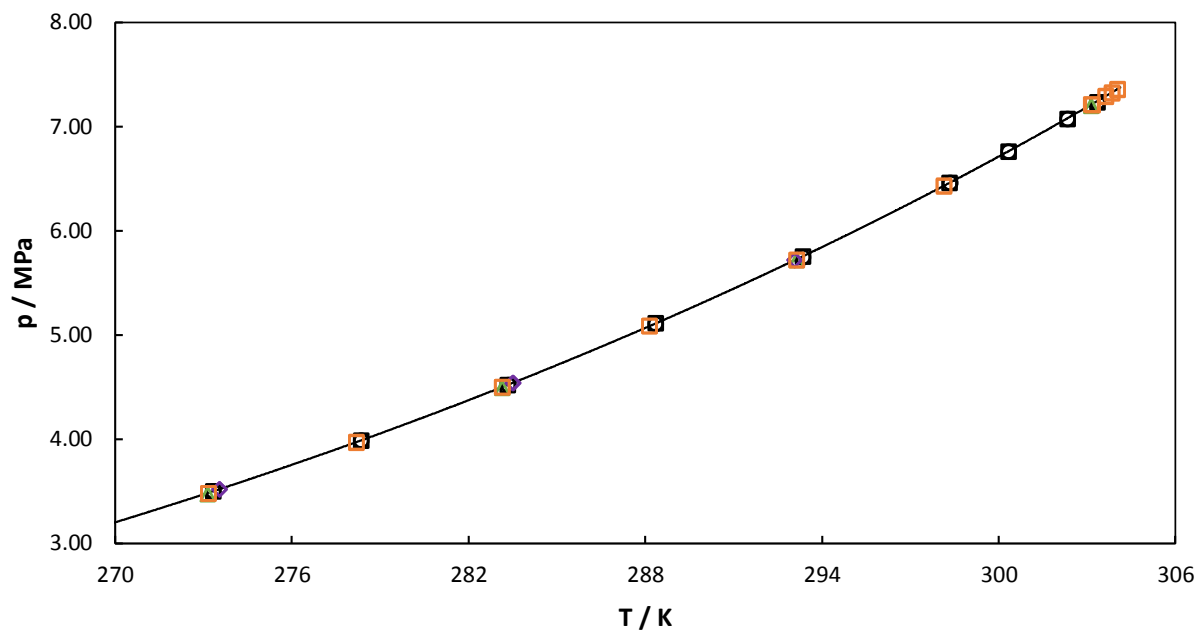

Figure 5.1 Experimental results in the temperature range of $273.35 \mathrm{~K}$ to $304.04 \mathrm{~K}$ of bubble points (o), and dew ( $\square$ ) for $\mathrm{CO}_{2}$, and their comparison with the literature Kim et al. [72] ( $\Delta$ ); Saleh et al. [73] ( $\left.\diamond\right)$; Gómez, [15] ( $\square$ ); reference from Span \& Wagner (-) [16].

Figure 5.2 shows graphically the percentage deviations of the experimental versus pressure data compared to the literature Kim et al. [72], Saleh et al. [73], and Gómez [15]. An analysis of the results obtained shows that the values returned by Kim et al. [72] return average percentage deviations of $\pm 0.17 \%$ using an equilibrium cell for the determination of these values, while the values proposed by Saleh et al. [73] return average percentage deviations $\pm 0.02 \%$ using a simple sinking density meter for the determination of densities. More recent values are those returned by Gómez [15]. using equipment similar to the one proposed in this work, which provides an average deviation of $\pm 0.03 \%$. In this work, an average percentage deviation obtained for bubble point was $0.07 \%$, while in the determination of dew point the percentage deviations obtained was $0.04 \%$. 


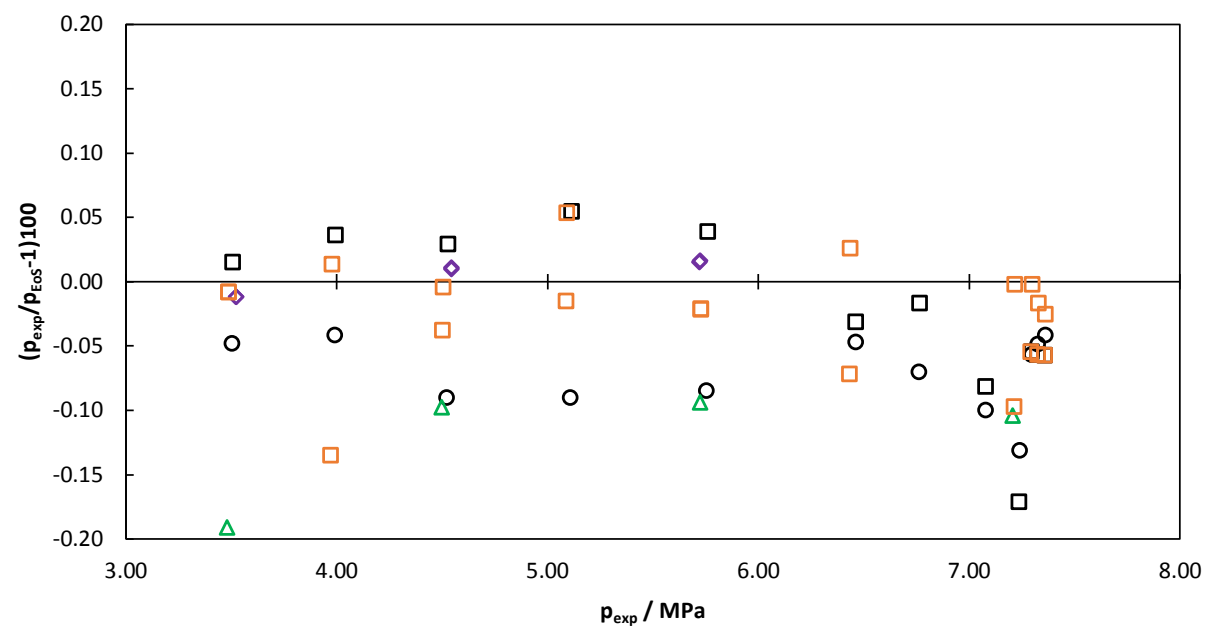

Figure 5.2 Percentage deviations of experimental $\mathrm{CO}_{2}$ data from bubble points $(\bullet)$, and dew points $(\boldsymbol{\square})$ for $\mathrm{CO}_{2}$,

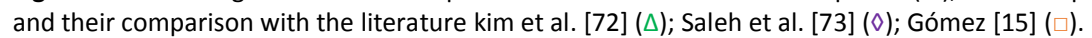

From the experimental data obtained from frequency we can make an approximation in the determination of the critical point of $\mathrm{CO}_{2}$. Table 5.2 shows the experimental results of pressure, temperature and resonance frequency in the vicinity of the critical point, and their comparison with the values returned by means of the equation of state Span \& Wagner [16].

Figure 5.3 shows the values in the temperature range near the critical point, compared to the $\mathrm{CO}_{2}$ state equation developed by Span \& Wagner [16] implemented in REFPROP software [20] and the literature provided by Kim et al. [72], andy Gómez, [15] In addition, it represents the reference value of the critical point that the equation of state described above returns. In order to determine the theoretical value of the critical point in this work, we have adjusted the experimental data of resonance frequency with respect to pressure and temperature to a second degree polynomial as shown in figure 5.6, the maximum of that polynomial will return the value of the resonance frequency of the critical point at, and therefore the values of the critical temperature or pressure. 


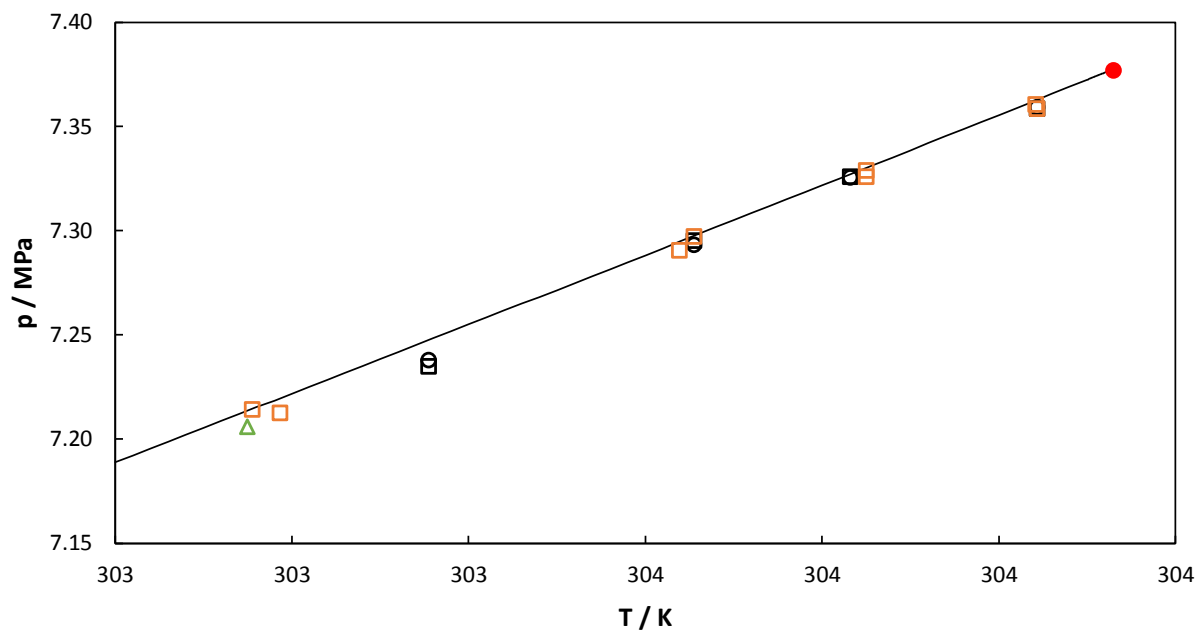

Figure 5.3 Experimental results in the vicinity of the $\mathrm{CO}_{2}$ critical point of the of bubble points (o), and dew ( $\square$ ) for $\mathrm{CO}_{2}$, and their comparison with the literature kim et al. [72] ( $\triangle$ ); Gómez, [15] ( $\square$ ). Datos desde la referencia de Span \& Wagner $(-)$ y punto crítico $(\bullet)[16]$.

Table 5.7 (Spanish version 102) shows the values obtained from the polarization densities of liquid and saturated vapour and the values of the dielectric constants calculated by means of the method established by Harvey and Lemmon [21].

Applying the method of perturbations described in section 3.4 was possible to give validity to the experimental technique described in this work, this method as already mentioned, relates the frequencies of resonances with the dielectric constant of the sample, in this work we refer to $\mathrm{CO}_{2}$, considering the initial state the saturated gas and the initial state the saturated liquid. Applying equation 3.35 and given that the values of the dielectric constant have been determined and that the relationship $V_{s} N_{c}$ can be determined from the geometry of the cavity, it is possible to obtain parameter " $A$ " analytically.

Figure 5.7 (Spanish version 103) shows the results obtained by applying equation 3.35. This proportionality parameter gives us the relationship between the resonance frequency and the dielectric constant (which depends on pressure 
and temperature), so that when approaching the critical point, the resonance frequencies are equalized making this parameter tend to zero.

\subsection{Analysis of the results}

In this section we have determined the vapour liquid equilibrium values of $\mathrm{CO}_{2}$ by determining the saturation pressures in the isothermal range up to the vicinity of the critical point, from the values of the resonance frequencies at each of the corresponding points. The experimental values have been compared with the Span \& Wagner [16] equation of state implemented in the NIST REFPROP software [20], and with the literature found Kim et al. [72], Saleh et al. [73], and Gómez, [15]. Average deviations of less than $0.07 \%$ have been obtained, values that are acceptable when compared with the literature and that are also below the uncertainty of the experimental equipment of $0.015 \mathrm{MPa}$ being $0.43 \%$ its lower limit of 3.502 MPa and $0.21 \%$ its upper limit of 7.077 MPa.

A study of the expanded uncertainties results in good results even in the theoretical determination of the critical point, which suggests that the procedure carried out for this determination has been adequate. As might be expected, the sources that contribute most are those due to the adjustment polynomial used for the theoretical determination of the critical point, and those due to the calibration of the measuring instruments used.

The value of the critical point has been determined by comparing the resonance frequencies with temperature and pressure, comparing the results obtained with those collected in the literature Gómez, [15], Gil et al. [74], Suehiro et al. [75] and Diefenbacher et al. [76], obtaining satisfactory results consistent with the uncertainty. 

CHAPTER 6

\section{Phase behaviour and}

\section{modelling of binary systems with $\mathrm{CO}_{2}$}





\section{Chapter 6. Phase behaviour and modelling of binary systems with $\mathrm{CO}_{2}$}

\subsection{Introduction}

The mixtures to be studied in this work are related to the purpose of this doctoral thesis and is to contribute with quality data in order to optimize the processes involved in the capture and storage of $\mathrm{CO}_{2}$ (CCS). For this purpose, six binary mixtures formed by $\mathrm{CH}_{4}, \mathrm{C}_{3} \mathrm{H}_{8}$ and $\mathrm{N}_{2}$ and different compositions of $\mathrm{CO}_{2}$ have been studied. All these mixtures have been studied in the range of temperature under the limits of the thermostatic bath that are established as lower limit 233.15 $\mathrm{K}$ until the zone of maximum temperature of each one of the systems (cricondentherm), the experimental measures have also been carried out in the proximities to the critical point and in the zones of retrograde condensation.

The experimental data obtained will first be compared with those returned by the GERG 2008 equation of state [4] where the deviations of the experimental data will be studied with those returned by this equation, in addition the behavior of the mixtures used in the elaboration of this thesis will be studied by means of the Peng-Robinson equation of state [17] using the Wong-Sandler mixing rules [18], where the adjustment parameters of the modelling of the experimental data will be obtained.

\subsection{Gravimetric samples preparation}

The mixtures investigated in this work have been prepared in the Spanish Centre of Metrology (CEM) according, according to ISO 6142:2011. The samples used were received with their corresponding calibration certificates where all the necessary information about their purity and their corresponding uncertainties is, the mixtures were supplied at an adequate pressure to prevent liquefaction of any 
of the compounds, in table 4.1 shows all the detailed information about purity according to their molar fraction and the uncertainty of these samples. In all the samples studied in this work the expanded uncertainty is expressed for a coverage factor $k=2$, the calculation of this uncertainty is based on the recommendations of the guide for the expression of uncertainty [11]. For the verification of the

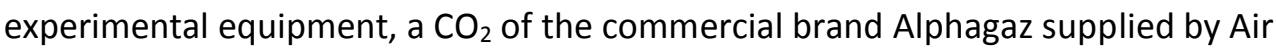
Liquid with a purity of $9.998 \%$ with an impurity content lower than $\leq 200 p p m$ in volume has been used.

\subsection{Experimental determination of the phase envelope}

In the case of fluid mixtures, it may happen that one of the components is separated from the mixture by density in the bottle., to avoid this event since it could alter the measurements, before connecting the bottle containing the sample in the equipment, it undergoes a mixing process by constant rotation, this process is carried out at room temperature and for a time of not less than $\mathbf{3 0}$ minutes. This ensures that the contents of the bottle are homogeneous when filling the syringe pumps.

By means of a procedure very similar to that described in sections 8.5.2 and 5.2, the experimental values of the bubble and dew points in each of the binary systems studied in this work have been determined. The vacuum times for cleaning the quipment and the waiting times for the selection of each of the isotherms have been respected.

The ISCO pumps have been filled to a sufficient pressure to allow the measurement to be carried out under the conditions in dew point and bubble point was recalculated using GERG 2008 equation of state [4] each isotherm and for the measurements started at a lower (dew point) or higher (bubble point) pressure of not less than $3 \mathrm{MPa}$ in the selected isotherm, in addition, a 30 minute wait shall be 
made for each point obtained to ensure that the entire sample is in the new phase and there is no possibility of metastable phases that alter the measurement. The flow rate that has been programmed and that will be supplied by the ISCO pumps be $0.05 \mathrm{mil} / \mathrm{min}$, this flow rate ensures that the appearance of the new phase occurs gradually for a better, causing a disturbance in the too high measurement that makes the VNA unable to carry out the necessary corrections and generates a computer error that ruins the time invested in the experiment.

\subsection{Uncertainties Determination}

As in section 6.4 of this paper, the combined expanded uncertainty measure has been determined in accordance with the "Guide to the expression of measurement uncertainty" (JCGM 106 2012) [11]. The measurement of the expanded uncertainty for temperature $U(T)$ in the measurement of dew points and bubble will be estimated using equation 8.5.1 explained in section 8.5.3.

The resonance frequency is normalised with a second-degree polynomial. This adjustment should be included as a contribution to the expanded uncertainty due to the adjustment polynomial $\mathrm{u}\left(\mathrm{p}_{\text {Pol }}\right)$ replacing $\mathrm{u}\left(\mathrm{p}_{\text {linear }}\right)$ in equation 5.3 . In addition, the contribution due to the composition must be taken into account in the measurement of the bubble or dew point $\mathrm{u}(\mathrm{p}(\mathrm{x}))$. has been taken into account for the determination of such uncertainty in the contribution due to the composition of the sample. 


\subsection{Experimental results}

Six binary systems have been measured at different $\mathrm{CO}_{2}$ compositions as described at the beginning of this chapter, using different isotherms in the temperature range from $233.15 \mathrm{~K}$ to the maximum temperature point (cricondentherm). The method of bubble and dew point has been used for the determination in the modelling of the experimental data applying the calculation of the fugacity by means of the equation of state proposed at the beginning of this chapter. The composition of the mixture and the temperature were used as starting data for the modelling, so that the pressures at each of the isotherms studied were calculated. A non-linear multiple regression procedure has been used, developed by Nelder \& Mead [78] and considering a modification of the objective function (equations 6.1 and 6.2), obtaining the minimum error according to the equation. A series of modifications specific to the program developed in Matlab by Martín et al. [79] was used. At the end of this chapter, a table is shown with the results of the modelizations of the systems studied in this work.

$$
\begin{aligned}
\text { Bubble point }(\mathrm{OF}) & =\sum_{1}^{\mathrm{N}}\left(\mathrm{p}_{\text {exp }}-\mathrm{p}_{\mathrm{cal}}\right)_{\mathrm{i}}^{2}+\sum_{1}^{\mathrm{N}}\left(\mathrm{y}_{\exp }-\mathrm{y}_{\mathrm{cal}}\right)_{\mathrm{i}}^{2} \\
\text { Dew point }(\mathrm{OF}) & =\sum_{1}^{\mathrm{N}}\left(\mathrm{p}_{\exp }-\mathrm{p}_{\mathrm{cal}}\right)_{\mathrm{i}}^{2}+\sum_{1}^{\mathrm{N}}\left(\mathrm{x}_{\exp }-\mathrm{x}_{\mathrm{cal}}\right)_{\mathrm{i}}^{2}
\end{aligned}
$$

Below are the experimental results of the phase envelopes for each of the systems studied in this work, the bubble and dew points were determined with respect to pressure variations over a series of isotherms. The results obtained were compared with those calculated using the GERG 2008 [4] equation of state, in addition to comparing the deviations obtained with the existing literature. Finally, 
the Peng-Robinson equation of state [17] was used using the Wong-Sandler mixing rules [18] to obtain the adjustment parameters for each of the systems.

Tables 6.3 to 6.8 shows the experimental values of the experimental systems studied in this work in molar fraction of carbon dioxide.

Figure 6.1 shows the phase envelope obtained experimentally for the system carbon dioxide + methane to a composition of 0.40064 in molar fraction of carbon dioxide, also in this figure has been represented the result of the modelling by Peng-Robinson EoS [17] using the Wong-Sandler mixing rules [18] as well as the curve calculated by means of the equation GERG 2008 [4], experimental values of different authors who have measured the liquid equilibrium steam of this system were included as well. The system was measured between the temperature range of (233.15 to 249.15) $\mathrm{K}$ and in the pressure range of (3.0 to 8.2) MPa including the retrograde zone considering the critical reference point calculated with the GERG2008 [4].

The combined expanded uncertainty for a coverage factor of $k=2$ in the temperature measurement was $U(T)=0.05 \mathrm{~K}$ and at the pressure measurement of $U(p)=0.010 \mathrm{MPa}$, while the relative uncertainty in the range of (3.051 to 8.177) $\mathrm{MPa}$ in the envelope was $\mathrm{U}_{\mathrm{r}}(\mathrm{p})=0.33 \%(\mathrm{MPa} / \mathrm{MPa})$ and $\mathrm{U}_{\mathrm{r}}(\mathrm{p})=0.12 \%(\mathrm{MPa} / \mathrm{MPa})$ respectively. 


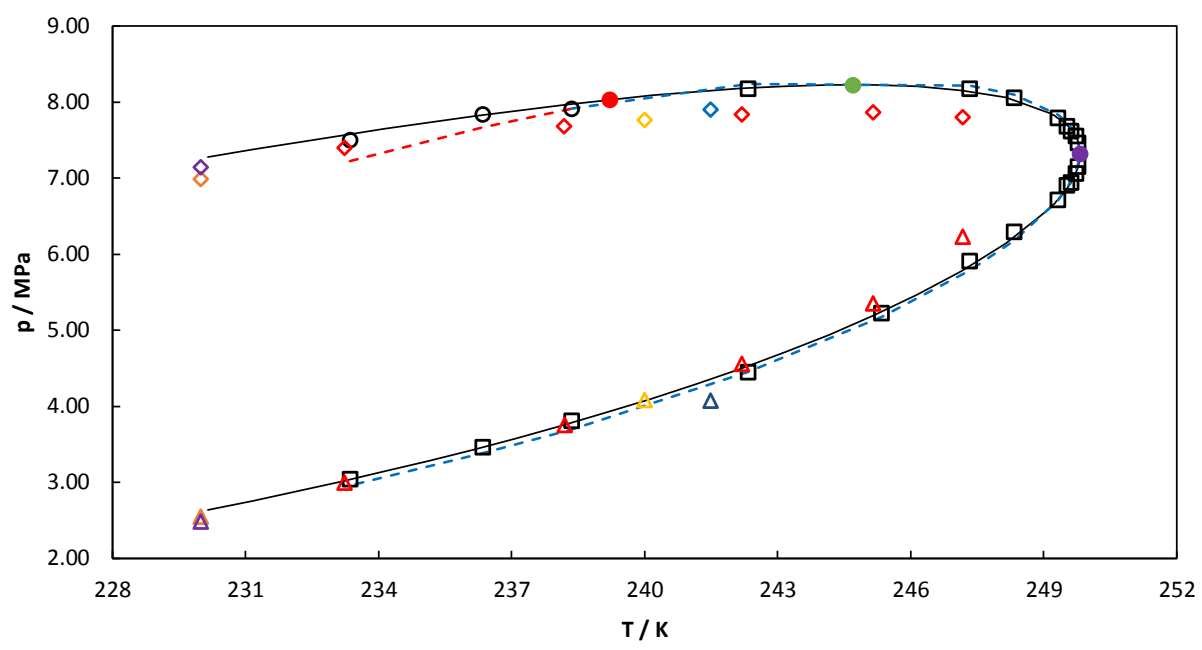

Figure 6.1 Phase envelope for the system $\left(\mathrm{x}_{1}\right) \mathrm{CO}_{2}+\left(1-\mathrm{x}_{1}\right) \mathrm{CH}_{4}\left(\mathrm{x}_{1}=0.40064\right)$ : bubble point $(\bullet)$ dew point $(\mathbf{\square})$ this PhD; fitting curve $(-)$, critical point $(\bullet)$, cricondentherm point $(\bullet)$, cricondenbar point (๑) GERG 2008 [4]; fitting curve Peng-Robinson [17] \& Wong-Sandler [18] bubble (- ), dew (--); Donnelly et al. [80] bubble ( 0$)$, dew $(\Delta)$; Wei et al. [81] bubble $(\diamond)$, dew $(\Delta)$; Webster et al. [82] bubble ( $\diamond)$, dew $(\Delta)$; Al-Sahhaf et al. [83] bubble $(\diamond)$, dew $(\triangle)$ and Gómez. [15] bubble $(\diamond)$, dew $(\Delta)$.

Figure 6.3 (Spanish version page 123) shows the percentage deviations with respect to equation GERG 2008 [4] compared to the temperature of the experimental data and the literature found. The figure shows how the percentage deviations obtained in this doctoral thesis do not exceed $2 \%$.

Figure 6.2. shows the figure of the phase envelope of the system carbon dioxide + methane to a composition of $\mathbf{0 . 6 0 1 6 2}$ in molar fraction of carbon dioxide studied, it shows the fit curve by GERG 2008 [4] in addition to the modelling of these experimental data by Peng-Robinson EoS [17] with Wong-Sandler mixing rules [18] obtaining the fit parameters of each of the systems which are described at the end of this chapter. the phase envelope for this new system was determined between the temperature range of (233.15 to 271.15 ) $\mathrm{K}$ and the pressure range of (1.8 to 8.9) $\mathrm{MPa}$ including the retrograde zone considering the critical reference point calculated with GERG 2008 [4]. 
The combined expanded uncertainties obtained have been determined with a coverage factor $k=2$, resulting in an uncertainty in the temperature measurement of $U(T)=0.05 \mathrm{~K}$ and in the pressure measurement of $U(p)=0.010 \mathrm{MPa}$, while the relative uncertainty in the range of (1.818 to 8.907) MPa in the enclosure was $U_{r}(p)$ $=0.55 \%(\mathrm{MPa} / \mathrm{MPa})$ and $\mathrm{U}_{\mathrm{r}}(\mathrm{p})=0.11 \%(\mathrm{MPa} / \mathrm{MPa})$ respectively.

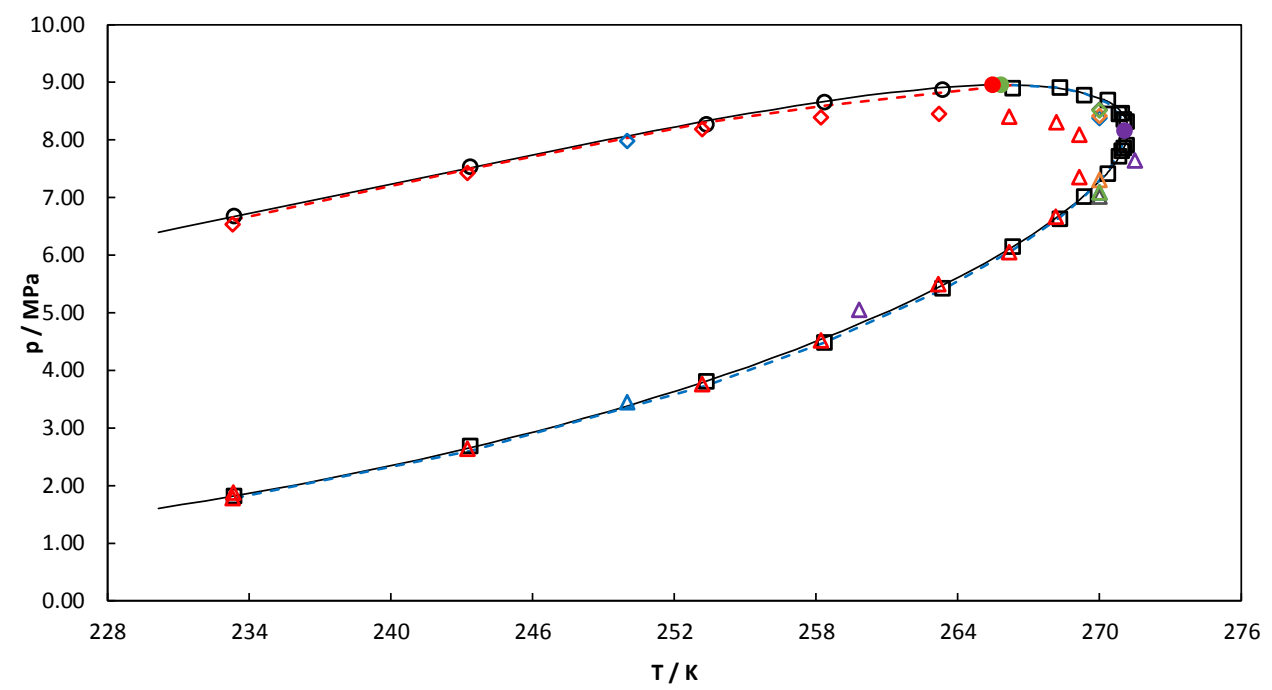

Figure 6.2 Phase envelope for the system $\left(x_{1}\right) \mathrm{CO}_{2}+\left(1-\mathrm{x}_{1}\right) \mathrm{CH}_{4}\left(\mathrm{x}_{1}=0.60162\right)$ : bubble point $(\bullet)$ dew point ( $\left.\square\right)$ this PhD; fitting curve $(-)$, critical point $(\bullet)$, cricondentherm point $(\bullet)$, cricondenbar point (๑) GERG 2008 [4]; fitting curve Peng-Robinson [17] \& Wong-Sandler [18] bubble (- -), dew (--); Donnelly et al. [80] dew ( $\Delta$ ); Wei et al. [81] bubble $(\diamond)$, dew $(\Delta)$; Webster et al. [82] bubble ( $\diamond)$, dew ( $\triangle$ ); Dávalos et al. [84] bubble $(\diamond)$, dew $(\Delta)$; Al-Sahhaf et al. [83] bubble $(\diamond)$, dew $(\Delta)$ and Gómez [15] bubble $(\diamond)$, dew $(\Delta)$.

Figure 6.5 (Spanish version page 128) shows the results of the percentage deviations from the experimental data studied in this doctoral thesis, as well as the deviations reported in the literature. In all cases, these deviations have been compared with the GERG 2008 [4]. The results of this mixture present an average percentage deviation of $0.30 \%$ for bubble point measurements, while for dew point measurements average deviations of $0.49 \%$ have been obtained. 
Figure 6.3 shows the experimental measurements of the phase envelope of the carbon dioxide + propane system at the composition of 0.60053 in molar fraction of carbon dioxide, this table shows the pressures at the bubble and dew points for each of the isotherms studied as well as the resonance frequency associated with each pressure and temperature in the temperature range of 229.15 to 329.60$) \mathrm{K}$ and in the pressure range of $(0.2$ to 6.8$) \mathrm{MPa}$ including the retrograde zone considering the critical reference point calculated with the GERG 2008 [4].

The combined expanded uncertainty for a coverage factor of $k=2$ in the temperature measurement was $\mathrm{U}(\mathrm{T})=0.05 \mathrm{~K}$ and in the pressure measurement of $U(p)=0.010 \mathrm{MPa}$, while the relative uncertainty in the range of $(0.227$ to 6.874$)$ $\mathrm{MPa}$ in the envelope was $\mathrm{U}_{\mathrm{r}}(\mathrm{p})=4.45 \%(\mathrm{MPa} / \mathrm{MPa})$ and $\mathrm{U}_{r}(\mathrm{p})=0.15 \%(\mathrm{MPa} / \mathrm{MPa})$ respectively. No metastable states were observed.

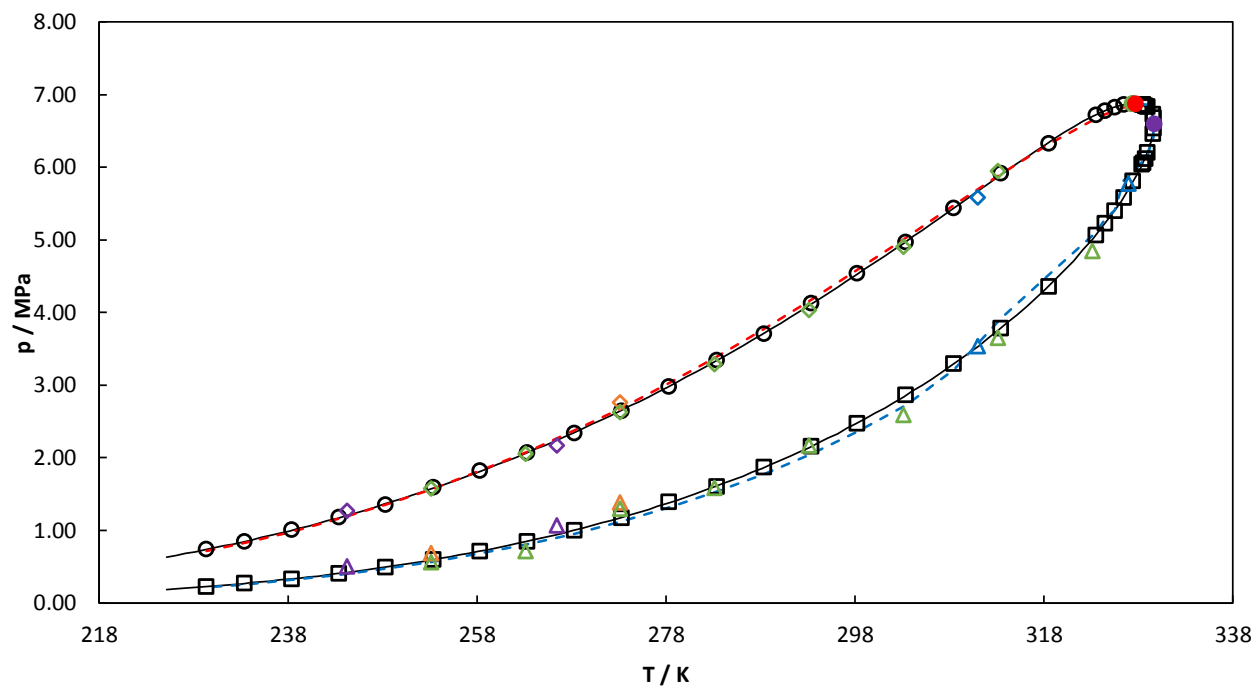

Figure 6.3 Phase envelope for the system $\left(x_{1}\right) \mathrm{CO}_{2}+\left(1-\mathrm{x}_{1}\right) \mathrm{C}_{3} \mathrm{H}_{8}\left(\mathrm{x}_{1}=0.60053\right)$ : bubble point $(\bullet)$ dew point $(\square)$ this PhD; fitting curve $(-)$, critical point $(\bullet)$, cricondentherm point $(\bullet)$, cricondenbar point $(\bullet)$ GERG 2008 [4]; fitting curve Peng-Robinson [17] \& Wong-Sandler [18] bubble $(--)$, dew (--); Niesen et al. [85] bubble ( $\diamond)$, $\operatorname{dew}(\Delta)$; Akers et al. [86] bubble $(\diamond)$, dew $(\Delta)$; Hamam et al. [87] bubble ( $\bullet)$, dew ( $\Delta)$; Kim et al. [72] bubble $(\diamond)$, $\operatorname{dew}(\Delta)$. 
Figure 6.7 (Spanish version page 134) (Spanish version page 123) shows the percentage deviations from the experimental data studied and their bibliographic comparison. the data presented for this system by means of this new measuring cell do not exceed $2 \%$.

Figure 6.4 shows the figure of the phase envelope, the curve calculated by the GERG 2008 [4] is also shown as well as the modelling of these experimental data by means of the Peng-Robinson EoS [17] with the Wong-Sandler mixing rules [18] obtaining the adjustment parameters for the system carbon dioxide + propane system to a composition of $\mathbf{0 . 7 9 7 7 7}$ in mole fraction of carbon dioxide. the phase envelope was determined between the temperature range of (233.15 to 310.36 ) $\mathrm{K}$ and the pressure range of (0.4 to 6.7) $\mathrm{MPa}$. Due to the proximity between the values of the maximum temperature point (cricondentherm), which for this composition is $310.44 \mathrm{~K}$, and the critical point, whose temperature value at the composition studied is $310.45 \mathrm{~K}$, no retrograde condensation zones has been detected.

The combined expanded uncertainty for a coverage factor of $k=2$ in the mean temperature was $U(T)=0.05 \mathrm{~K}$ and in the pressure measure of $U(p)=0.010 \mathrm{MPa}$, while the relative uncertainty in the range of (0.497 to 6.772) MPa in the envelope was $U_{r}(p)=2.01 \%(\mathrm{MPa} / \mathrm{MPa})$ and $U_{r}(p)=0.15 \%(\mathrm{MPa} / \mathrm{MPa})$ respectively. 


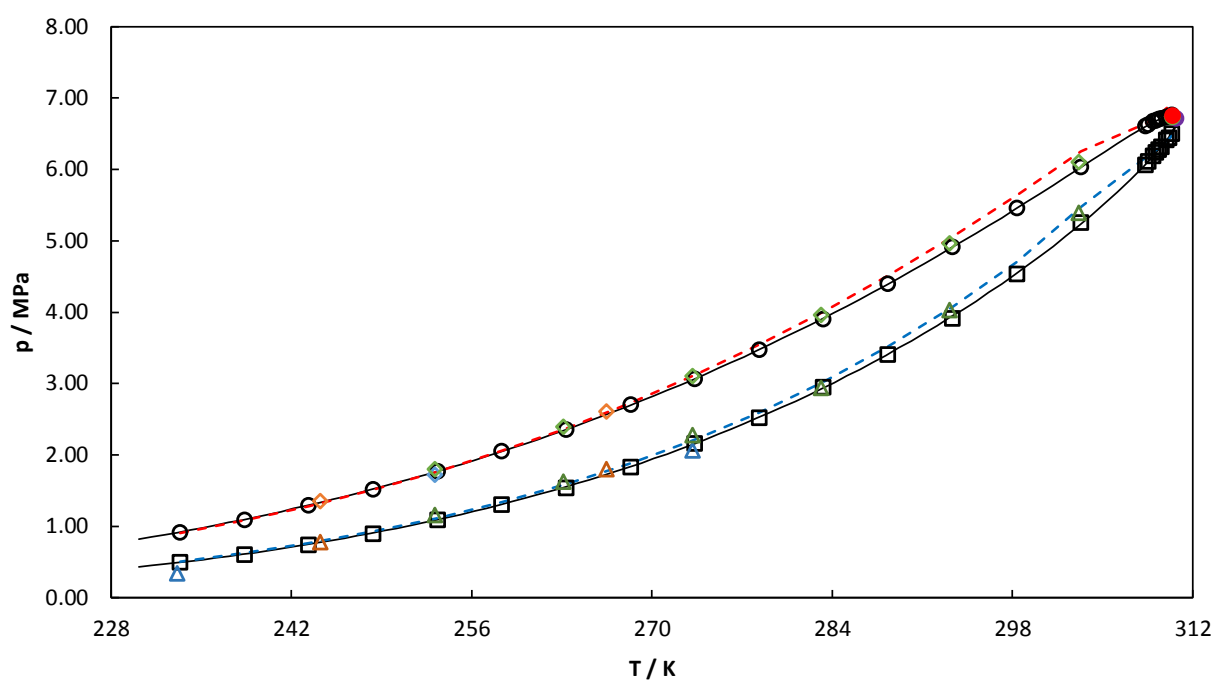

Figure 6.4 Phase envelope for the system $\left(\mathrm{x}_{1}\right) \mathrm{CO}_{2}+\left(1-\mathrm{x}_{1}\right) \mathrm{C}_{3} \mathrm{H}_{8}\left(\mathrm{x}_{1}=0.79777\right)$ : bubble point $(\bullet)$ dew point $(\mathbb{\square})$ this PhD; fitting curve (-), critical point $(\bullet)$, cricondentherm point $(\bullet)$, cricondenbar point $(\bullet)$ GERG 2008 [4]; fitting curve Peng-Robinson [17] \& Wong-Sandler [18] bubble (- ), dew (--); Akers et al. [86] bubble ( $\diamond)$, dew $(\Delta)$; Hamam et al. [87] bubble $(\diamond)$, dew $(\triangle)$; Kim et al. [72] bubble $(\diamond)$, dew $(\Delta)$.

Figure 6.9 (Spanish version page 123) shows the percentage deviations from the experimental data studied and their bibliographic comparison. For this system, the mean percentage deviations of the obtained experimental data do not exceed $0.7 \%$.

Figure 6.5 shows the figure of the phase envelope, the curve calculated by the GERG 2008 [4] is also shown as well as the modelling of these experimental data by means of the Peng-Robinson EoS [17] with the Wong-Sandler mixing rules [18] obtaining the adjustment parameters for the carbon dioxide + nitrogen system to a composition of $\mathbf{0 . 4 9 9 0 5}$ in mole fraction of carbon dioxide. The system was measured between the temperature range of (229.37 to 262.63) $\mathrm{K}$ and the pressure range of (1.9 to 18.8) MPa including the retrograde zone considering the critical reference point.

The combined expanded uncertainty for a coverage factor of $k=2$ in the temperature measurement was $\mathrm{U}(\mathrm{T})=0.05 \mathrm{~K}$ and in the pressure measurement of $U(p)=0.010 \mathrm{MPa}$, while the relative uncertainty in the range of (1.993 to 18.790) 
$\mathrm{MPa}$ in the envelope was $\mathrm{U}_{\mathrm{r}}(\mathrm{p})=0.50 \%(\mathrm{MPa} / \mathrm{MPa})$ and $\mathrm{U}_{\mathrm{r}}(\mathrm{p})=0.05 \%(\mathrm{MPa} / \mathrm{MPa})$ respectively.

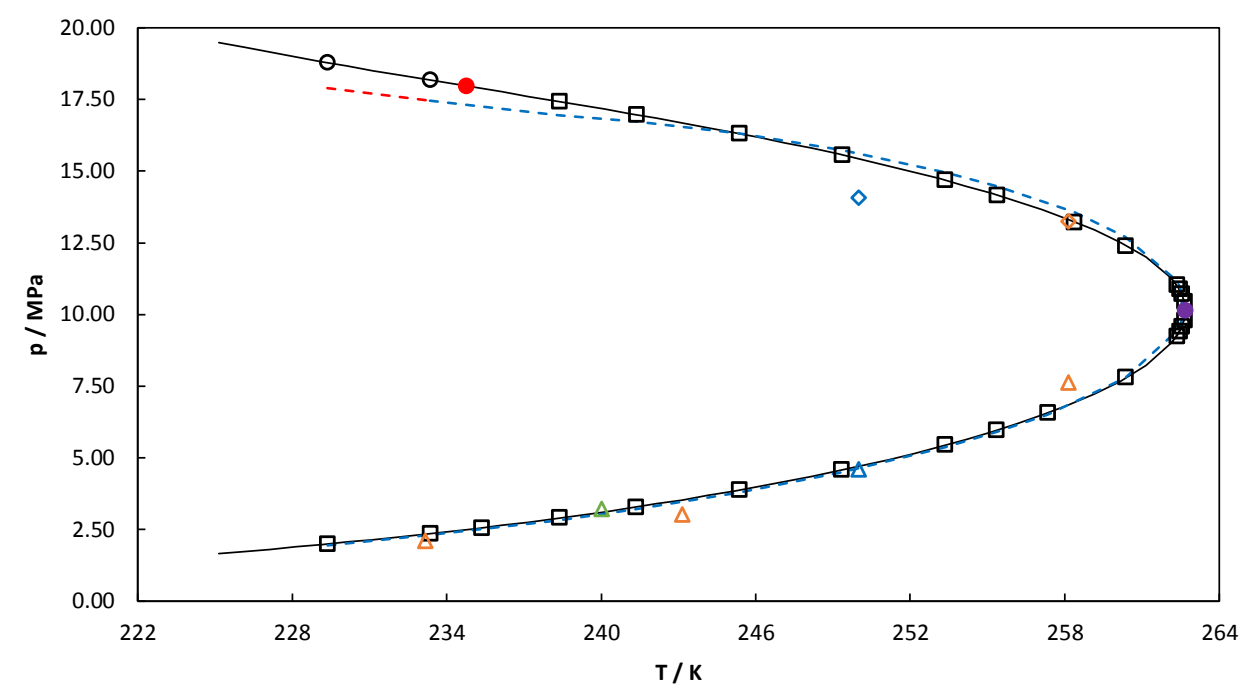

Figure 6.5 Phase envelope for the system $\left(\mathrm{x}_{1}\right) \mathrm{CO}_{2}+\left(1-\mathrm{x}_{1}\right) \mathrm{N}_{2}\left(\mathrm{x}_{1}=0.49905\right)$ : bubble point $(\bullet)$ dew point $(\boldsymbol{\square})$ this

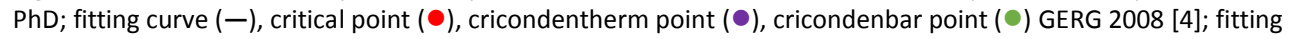
curve Peng-Robinson [17] \& Wong-Sandler [18] bubble (- ), dew (--); Brown et al. [88] bubble ( $\diamond)$, dew $(\Delta)$; Fandiño et al. [89] bubble $(\diamond)$, dew $(\triangle)$; Al Sahhaf et al. [83] dew $(\Delta)$.

Figure 6.11 (Spanish version page 144) shows the percentage deviations from the temperature of the experimental data, and the literature found shows that, although the literature found is scarce, the percentage deviations obtained so far are the lowest. The deviations of GERG 2008 [4] from the data with this new measuring cell do not exceed $0.6 \%$.

Figure 6.6 shows the figure of the phase envelope, the curve calculated by the GERG 2008 [4] is also shown as well as the modelling of these experimental data by means of the Peng-Robinson EoS [17] with Wong-Sandler mixing rules [18] obtaining the adjustment parameters for the carbon dioxide + nitrogen system to a composition of $\mathbf{0 . 7 9 7 7 7}$ in mole fraction of carbon dioxide, in the temperature 
range (229.36 to 287.33$) \mathrm{K}$ and in the pressure range (1.1 to 12.1$) \mathrm{MPa}$ including the retrograde zone considering the critical point of reference.

The combined expanded uncertainty for a coverage factor of $k=2$ in the temperature measurement was $\mathrm{U}(\mathrm{T})=0.05 \mathrm{~K}$ and in the pressure measurement of $U(p)=0.010 \mathrm{MPa}$, while the relative uncertainty in the range of (1.126 to 12.061) $\mathrm{MPa}$ in the envelope was $\mathrm{U}_{r}(p)=0.89 \%(\mathrm{MPa} / \mathrm{MPa})$ and $\mathrm{U}_{r}(p)=0.08 \%(\mathrm{MPa} / \mathrm{MPa})$ respectively.

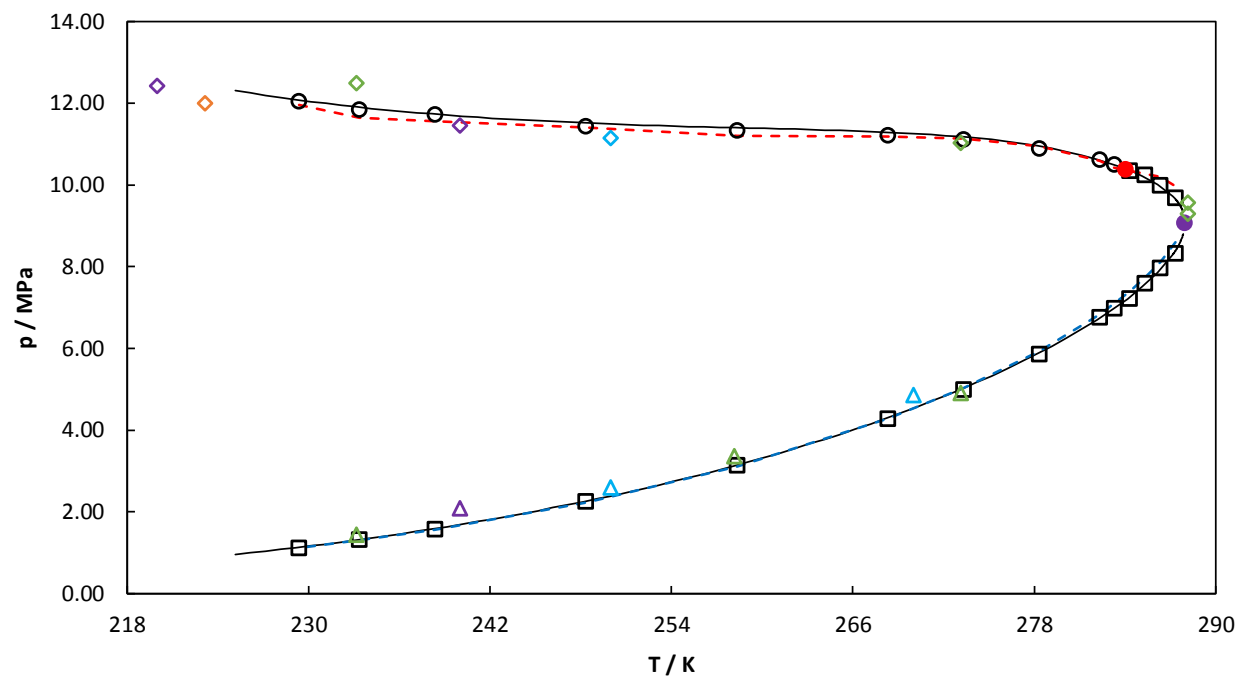

Figure 6.5 Phase envelope for the system $\left(x_{1}\right) C O_{2}+\left(1-x_{1}\right) N_{2}\left(x_{1}=0.79777\right)$ : bubble point $(\bullet)$ dew point $(\square)$ this

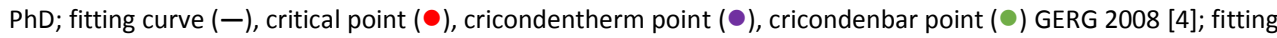
curve Peng-Robinson [17] \& Wong-Sandler [18] bubble (- ), dew (--); Brown et al. [88] bubble ( $\diamond)$, dew $(\Delta)$; Westman et al. [90] bubble ( $\diamond)$; Fandiño et al. [89] bubble $(\diamond)$, dew $(\Delta)$; Al Sahhaf et al. [83] bubble $(\diamond)$, dew $(\Delta)$.

Figure 6.13 (Spanish version page 148) shows the results of the percentage deviations from the experimental data studied in this doctoral thesis as well as the deviations from the data in the literature, these deviations have been compared in all cases with the GERG 2008 [4]. As in the previous system, the percentage deviations of the experimental data do not exceed $0.8 \%$. 


\subsection{Analysis of the results}

Six systems containing carbon dioxide to different compositions in this substance have been studied in this work, the phase envelope has been determined in a wide range of pressures and temperatures and the results obtained have been compared by means of the equation of state GERG 2008 [4] where the deviations of the experimental data were studied with those returned by this equation, In addition, the behaviour of the mixtures used in the preparation of this thesis was studied using the Peng-Robinson cubic state equation [15] using the Wong-Sandler mixing rules [16], where the adjustment parameters of the experimental data modelling will be obtained.

Table 6.9 (Spanish version page 149) shows a summary of the uncertainties, firstly, it is observed that regardless of the system in question, the uncertainty due to temperature does not vary, this is due to the fact that the first place is due to the great stability of the probes used and secondly to the meticulous calibration procedure used to determine the uncertainty of these. With respect to the uncertainties due to pressure, it can be seen that the values are within the values consistent with the resolution of the measuring instrument. Due to the procedure used in the determination of the experimental points, the greatest contribution to the uncertainty in the measurement of pressure is due to the contribution to the composition of the sample.

Table 6.11 (Spanish version page 151) shows the results of modelling using the Peng-Robinson cubic state equation [15] using the Wong-Sandler mixing rules [16]. It has been chosen as non-adjustable parameter that defines the local composition (NRTL) [75] where it is recommended that the values in non-symmetric systems be between 0.20 and 0.55 . Similarly considering the non-association between the mixtures used in this work, the value of 0.30 has been chosen for this parameter to model the systems with methane and nitrogen, the system containing propane 
has been better adjusted to a value of 0.47 presumably to the high asymmetry between the two molecules.

It is observed that the mean percentage deviations are significantly low offering a good fit with the experimental data, even in the vicinity of the critical point. Figures 6.14 to 6.19 show the percentage deviations of the systems studied compared to the equation of state with respect to Peng-Robinson EoS [17] using Wong-Sandler mixing rules [18]. As can be expected, although the deviations from pressure in general are good, the experimental data observed individually offer some deviation, especially if we compare this adjustment with that offered by GERG 2008 [4], this is due to the inherent complexity of the equation of state, although both are based on Helmholtz energies, the thermodynamic adjustment parameters used GERG 2008 [4] are much greater than those used in the PengRobinson EoS [17] using Wong-Sandler mixing rules [18]. In general, the percentage deviations of the systems studied using this equation of state are not greater than $6 \%$. 


\section{CHAPTER}

\section{Conclusions}





\section{Chapter 7. Conclusions}

\subsection{Introduction}

The main objective of this doctoral thesis has been the development of an experimental technique, of maximum accuracy, for the determination of thermodynamic properties, in this case, the phase envelope in the region of the critical point. The starting point was a cylindrical cavity resonant in microwave proposed by Gómez [15], which has undergone a series of modifications and improvements, including design plans, construction in a mechanical workshop, assembly of all elements in the laboratory, calibration of instrumentation and validation with a test compound.

The selected substance, whose thermodynamic properties are well known, was carbon dioxide. The Span \& Wagner [16] is able to predict its behaviour.

The equipment has been automated in order to improve data collection, this has been achieved by creating programs to program the pressure and temperature ramps needed at each experimental point and that allow to relate the frequency of resonance with the pressure variation in the change of phase accurately.

The new equipment has succeeded in improving the repeatability of measurements and a reduction in measurement uncertainty.

The measurements carried out provide experimental data for the study of the vapour liquid equilibrium of binary systems, formed by components of energetic gases such as biogas. These components have been $\mathrm{CO}_{2}, \mathrm{CH}_{4}, \mathrm{C}_{3} \mathrm{H}_{8}$ y $\mathrm{N}_{2}$. 
The work carried out has led to the following conclusions:

1. A new microwave cylindrical cavity has been designed and constructed, based on the experience acquired with the equipment available in the laboratory, improving the water tightness of the sapphire tube and improving the quality of the resonance signal, this has enabled both measurement times and uncertainties to be reduced.

2. The $\mathrm{CO}_{2}$ vaporization curve has been determined from $3.5 \mathrm{MPa}$ to the critical point, with a temperature uncertainty of $0.05 \mathrm{~K}$ and $0.006 \mathrm{MPa}$ in pressure. The maximum deviation from the Span \& Wagner [16] has been less than $0.1 \%$ in pressure and less than the measurement uncertainty. The coordinates of the critical point differ by $0.04 \mathrm{~K}$ and $0.010 \mathrm{MPa}$ from the Span \& Wagner [16] with an expanded experimental uncertainty of $0.06 \mathrm{~K}$ and $0.010 \mathrm{MPa}$ respectively.

3. The phase envelope of three binary systems containing $\mathrm{CO}_{2},\left(\mathrm{CO}_{2}+\mathrm{CH}_{4}\right)$, $\left(\mathrm{CO}_{2}+\mathrm{C}_{3} \mathrm{H}_{8}\right)$ and $\left(\mathrm{CO}_{2}+\mathrm{N}_{2}\right)$, in the temperature range of $229.15 \mathrm{~K}$, has been determined at two different compositions in molar fraction of $\mathrm{CO}_{2}$, up to the point of maximum temperature (cricondentherm), characteristic of each system and composition studied, including the retrograde condensation zone and the critical point.

4. These systems have been modelled using the Peng-Robinson EoS [17] using the Wong-Sandler mixing rules [18] and a comparison of the experimental data with the GERG 2008 [4] has been made, the average differences have been for the system $(0.4) \mathrm{CO}_{2}+(0.6) \mathrm{CH}_{4}$ was $0.05 \%$, for the system (0.6) $\mathrm{CO}_{2}+(0.4) \mathrm{CH}_{4}$ was $0.27 \%$, for the system $(0.6) \mathrm{CO}_{2}+(0.4) \mathrm{C}_{3} \mathrm{H}_{8}$ was $0.03 \%$, for the $0.04 \%(0.8) \mathrm{CO}_{2}+(0.2) \mathrm{C}_{3} \mathrm{H}_{8}$ system, for the $0.08 \%$ $(0.5) \mathrm{CO}_{2}+(0.5) \mathrm{N}_{2}$ system and for the $0.20 \%(0.8) \mathrm{CO}_{2}+(0.2) \mathrm{N}_{2}$ system. Comparisons with existing literature have been made for each system. 
5. All the condensation points retrograde to the isotherms selected in each of the systems studied have been precisely determined. No retrograde condensation points were found in the $(0.8) \mathrm{CO}_{2}+(0.2) \mathrm{C}_{3} \mathrm{H}_{8}$ system because the critical point and cricondentherm point, $310.45 \mathrm{~K}$ and $310.71 \mathrm{~K}$ respectively, are very close together.

6. An exhaustive study of the contribution to uncertainty of each of the systems studied has been carried out, obtaining the combined expanded uncertainties $(k=2)$ in the measurement of temperature and pressure at both the bubble and dew points. For the system $(0.4) \mathrm{CO}_{2}+(0.6) \mathrm{CH}_{4}$ have been of $U(T)=0.05 \mathrm{~K}$, and $U(p)=0.010 \mathrm{MPa}$, for the system (0.6) $\mathrm{CO}_{2}+(0.4) \mathrm{CH}_{4}$ have been of $\mathrm{U}(\mathrm{T})=0.05 \mathrm{~K}$, and $\mathrm{U}(\mathrm{p})=0.010 \mathrm{MPa}$, for the system $(0.6) \mathrm{CO}_{2}+(0.4) \mathrm{C}_{3} \mathrm{H}_{8}$ have been of $U(T)=0.05 \mathrm{~K}$, and $U(p)=0.010$ $\mathrm{MPa}$, for the system $(0.8) \mathrm{CO}_{2}+(0.2) \mathrm{C}_{3} \mathrm{H}_{8}$ have been of $\mathrm{U}(\mathrm{T})=0.05 \mathrm{~K}$, and $U(p)=0.010 \mathrm{MPa}$, for the system $(0.5) \mathrm{CO}_{2}+(0.5) \mathrm{N}_{2}$ have been of $U(T)=0.05$ $\mathrm{K}$, and $\mathrm{U}(\mathrm{p})=0.010 \mathrm{MPa}$ and for the system $(0.8) \mathrm{CO}_{2}+(0.2) \mathrm{N}_{2}$ have been of $U(T)=0.05 \mathrm{~K}$, and $\mathrm{U}(\mathrm{p})=0.010 \mathrm{MPa}$.

7. Finally, and by way of summary, this thesis has provided knowledge of the phase envelopes of binary systems of energy gas components and has developed an experimental technique capable of providing accurate data on the vapour liquid equilibrium at high pressures. 



\section{Bibliography}





\section{Bibliography}

[1] United Nations, Framework Convention on Climate, (2015) 31.

[2] R.K. Pachauri, L. Meyer, Intergovernmental Panel on Climate Change, Clim. Chang. Synth. Rep. (2014) 2-26. doi:10.1088/1751-8113/44/8/085201.

[3] B. Page, The global status of CCS 2014, Summ. Rep. (2016) 1-28.

[4] O. Kunz, W. Wagner, The GERG-2008 wide-range equation of state for natural gases and other mixtures: An expansion of GERG-2004, J. Chem. Eng. Data. 57 (2012) 3032-3091. doi:10.1021/je300655b.

[5] R. Privat, J.N. Jaubert, Comments on "PVTxy properties of $\mathrm{CO}_{2}$ mixtures relevant for $\mathrm{CO}_{2}$ capture, transport and storage: Review of available experimental data and theoretical models," Appl. Energy. 93 (2012) 750752. doi:10.1016/j.apenergy.2011.09.012.

[6] B. Flach, K. Bendz, R. Krautgartner, S. Lieberz, M.E. Smith, EU Biofuels Annual, Glob. Agric. Inf. Netw. (2014) 1-34.

[7] B. Flach, S. Lieberz, M. Rondon, B. Williams, C. Teiken, EU Biofuels Annual, Glob. Agric. Inf. Netw. (2015) 1-42.

[8] T. Abbasi, S.M. Tauseef, S.A. Abbasi, Biogas energy, 2012. doi:10.1007/9781-4614-1040-9.

[9] EurObserv'ER, Biogas Barometer, (2017).

[10] W.J. Rogers, J.C. Holste, P.T. Eubank, K.R. Hall, Microwave apparatus for phase transition studies of corrosive fluids to $1.7 \mathrm{kbar}$ and $588 \mathrm{~K}$, Rev. Sci. Instrum. 56 (1985) 1907-1912. doi:10.1063/1.1138443.

[11] F. Fogh, P. Rasmussen, Detection of high-pressure dew and bubble points using a microwave technique, Ind. Eng. Chem. Res. 28 (1989) 371-375. doi:10.1021/ie00087a018.

[12] M.D. Frørup, J.T. Jepsen, A. Fredenslund, P. Rasmussen, High pressure dew and bubble points from microwave measurements, Fluid Phase Equilib. 52 
(1989) 229-235. doi:10.1016/0378-3812(89)80329-2.

[13] A.R.H. Goodwin, M.D. Frørup, E.H. Stenby, Microwave detection of dew points: results for complex mixtures, J. Chem. Thermodyn. 23 (1991) 713715. doi:10.1016/S0021-9614(05)80189-2.

[14] A.R.H. Goodwin, J.B. Mehl, M.R. Moldover, Reentrant radio-frequency resonator for automated phase-equilibria and dielectric measurements in fluids, Rev. Sci. Instrum. 67 (1996) 4294-4303. doi:10.1063/1.1147580.

[15] A. Gómez, Desarrollo de una nueva técnica experimental de medida de equilibrio de fases a alta presión basada en una cavidad cilíndrica en microondas con aplicación al biogás, Tesis Doctoral. Universidad de Valladolid, 2015.

[16] R. Span, W. Wagner, A new equation of state for carbon dioxide covering the fluid region from the triple-point temperature to $1100 \mathrm{~K}$ at pressures up to $800 \mathrm{MPa}$, J. Phys. Chem. Ref. Data. 25 (1996) 1509-1596. doi:10.1063/1.555991.

[17] D.Y. Peng, D.B. Robinson, A new two-constant equation of state, Ind. Eng. Chem. Fundam. 15 (1976) 59-64. doi:10.1021/i160057a011.

[18] D.S.H. Wong, S.I. Sandler, A theoretically correct mixing rule for cubic equations of state, AIChE J. 38 (1992) 671-680. doi:10.1002/aic.690380505.

[19] J. Committee for Guides in Metrology, Evaluación de datos de medición. Guía para la expresión de la incertidumbre de medida, (2012) 1-142. doi:10.1515/ci.2013.35.2.22.

[20] NIST, NIST standard reference database 23: reference fluid thermodynamic and transport properties (REFPROP), Version 9.1, (2013). doi:10.1234/12345678.

[21] A. Harvey, E. Lemmon, Method for estimating the dielectric constant of natural gas mixtures, Int. J. Thermophys. 26 (2005) 31-46. doi:10.1007/s10765-005-2351-5. 
[22] H.D. Young, R.A. Freedman, A.L. Ford, Física Universitaria Vol.1, Pearson Educ. (2009) 760.

[23] H.D. Young, R.A. Freedman, A.L. Ford, Física Universitaria Vol.2, Pearson Educ. 2 (2009) 708.

[24] J.W. Gibbs, The scientific papers of J Willard Gibbs, Nature. 75 (1907) 361362. doi:10.1038/075361a0.

[25] G.N. Lewis, M. Randall, Thermodynamics and the free energy of chemical substances, Mc Graw-Hill, 1923.

[26] J.M. Prausnitz, Molecular thermodynamics of fluid-phase equilibria, Prentice Hall. (1969) 158-159. doi:10.1016/0021-9614(70)90078-9.

[27] J.M.S. Fonseca, R. Dohrn, S. Peper, High-pressure fluid-phase equilibria: Experimental methods and systems investigated (2005-2008), Fluid Phase Equilib. 300 (2011) 1-69. doi:10.1016/j.fluid.2010.09.017.

[28] P.H.V. Konynenburg, R.L. Scott, Critical lines and phase equilibria inbBinary Van Der Waals mixtures, Philos. Trans. R. Soc. A Math. Phys. Eng. Sci. 298 (1980) 495-540. doi:10.1098/rsta.1980.0266.

[29] E.D. Silverman, G. Thodos, Cricondentherms and cricondenbars their prediction for binary hydrocarbon systems, Ind. Eng. Chem. Fundam. 1 (1962) 299-303. doi:10.1021/i160004a013.

[30] M.B. King, Phase equilibrium in mixtures, Elsevier Science, 1969.

[31] L.F. Chen, C.K. Ong, C.P. Neo, V. V. Varadan, V.K. Varadan, Microwave Electronics. Measurement and Materials Characterization, John Wiley \& , Ltd, 2004. doi:10.1002/0470020466.

[32] A.P. Gregory, R.N. Clarke, A review of RF and microwave techniques for dielectric measurements on polar liquids, IEEE Trans. Dielectr. Electr. Insul. 13 (2006) 727-743. doi:10.1109/TDEI.2006.1667730.

[33] L.R. Victoriano, M.L.M. del Mar, C.P. Castro, Electromagnetismo II, Universidad Nacional de Educación a Distancia Madrid, 2013. 
[34] A. Tamburrano, D. Desideri, A. Maschio, S.S. Maria, Coaxial waveguide methods for shielding effectiveness measurement of planar materials up to $18 \mathrm{GHz}$, IEEE Trans. Electromagn. Compat. 56 (2014) 1386-1395. doi:10.1109/TEMC.2014.2329238.

[35] Agilent Technologies, Understanding the fundamental principles of vector network analysis, Appl. Note 5965-7707E. (2012) 15. http://www.agilent.com/.

[36] P. Atkins, J. De Paula, Physical chemistry, W.H. Freeman nd Company, 2009. doi:10.1021/ed056pA260.1.

[37] R.E. Fornari, P. Alessi, I. Kikic, High pressure fluid phase equilibria: Experimental methods and systems investigated (1978-1987), Fluid Phase Equilib. 57 (1990) 1-33. doi:10.1016/0378-3812(90)80010-9.

[38] M. Christov, R. Dohrn, High-pressure fluid phase equilibria: Experimental methods and systems investigated (1994-1999), Fluid Phase Equilib. 202 (2002) 153-218. doi:10.1016/S0378-3812(02)00096-1.

[39] R. Dohrn, S. Peper, J.M.S. Fonseca, High-pressure fluid-phase equilibria: Experimental methods and systems investigated (2000-2004), Fluid Phase Equilib. 288 (2010) 1-54. doi:10.1016/j.fluid.2009.08.008.

[40] E.F. May, Measurement of the thermodynamic properties of single phases. IUPAC Experimental Thermodynamics, Volume VI, 2003. doi:10.1021/je600558y.

[41] D.S. Tsiklis, Handbook of techniques in high pressure research and engineering, plenum press, 1968. doi:10.1007/978-1-4684-8360-4.

[42] R.. Weir, T.W. De Loos, Measurement of the thermodynamic properties of multiple phases, Experimental Thermodynamics vol VII, Elsevier Ltd, 2005.

[43] M.N. Afsar, J.R. Birch, R.N. Clarke, G.W. Chantry, The measurement of the properties of materials, Proc. IEEE. 74 (1986) 183-199. doi:10.1109/PROC.1986.13432. 
[44] J. Asmussen, R. Mallavarpu, J.R. Hamann, The design of a microwave plasma cavity, Proc. IEEE. 62 (1974) 109-117. doi:10.1109/PROC.1974.9391.

[45] B.A. Younglove, G.C. Straty, A capacitor for accurate wide range dielectric constant measurements on compressed fluids, Rev. Sci. Instrum. 41 (1970) 1087-1089. doi:10.1063/1.1684699.

[46] G.C. Straty, B.A. Younglove, Compact capacitor for wide-range dielectric constant measurements on small amounts of fluids, Rev. Sci. Instrum. 50 (1979) 1309-1310. doi:10.1063/1.1135702.

[47] A.R.H. Goodwin, M.R. Moldover, Phase border and density determinations in the critical region of (carbon dioxide + ethane) determined from dielectric permittivity measurements, J. Chem. Thermodyn. 29 (1997) 1481-1494. doi:10.1006/jcht.1997.0257.

[48] E.F. May, T.J. Edwards, A.G. Mann, C. Edwards, R.C. Miller, Development of an automated phase behaviour measurement system for lean hydrocarbon fluid mixtures, using re-entrant rf/microwave resonant cavities, Fluid Phase Equilib. 185 (2001) 339-347. doi:10.1016/S0378-3812(01)00482-4.

[49] E.F. May, T.J. Edwards, A.G. Mann, C. Edwards, An improved microwave apparatus for phase behaviour measurements in lean gas condensate fluids, Fluid Phase Equilib. 215 (2004) 245-252. doi:10.1016/j.fluid.2003.08.015.

[50] E.F. May, T.J. Edwards, A.G. Mann, C. Edwards, Dew point, liquid volume, and dielectric constant measurements in a vapor mixture of methane + propane using a microwave apparatus, Int. J. Thermophys. 24 (2003) 15091525.

[51] P.H. Huang, D.C. Ripple, M.R. Moldover, G.E. Scace, A Reference standard for measuring humidity of air using a re-entrant radio frequency resonator, Natl. Inst. Stand. Technol. Gaithersburg, Maryland, U.S.A. (2006) 2-5.

[52] M.E. Kandil, K.N. Marsh, A.R.H. Goodwin, A re-entrant resonator for the 
measurement of phase boundaries: Dew points for $\left\{0.4026 \mathrm{CH}_{4}+0.5974\right.$ $\left.\mathrm{C}_{3} \mathrm{H}_{8}\right\}$, J. Chem. Thermodyn. 37 (2005) 684-691. doi:10.1016/j.jct.2004.11.004.

[53] M.E. Kandil, K.N. Marsh, A.R.H. Goodwin, Determination of the relative permittivity and density within the gas phase and liquid volume fraction formed within the two-phase region for $\left(0.4026 \mathrm{CH}_{4}+0.5974 \mathrm{C}_{3} \mathrm{H}_{8}\right)$ with a radio frequency re-entrant cavity, J. Chem. Eng. Data. 52 (2007) 1660-1671. doi:10.1021/je700053u.

[54] R.J. Underwood, R. Cuccaro, S. Bell, R.M. Gavioso, D.M. Ripa, M. Stevens, M. De Podesta, A microwave resonance dew-point hygrometer, Meas. Sci. Technol. 23 (2012) 1-9. doi:10.1088/0957-0233/23/8/085905.

[55] M.B. Ewing, D.D. Royal, A highly stable cylindrical microwave cavity resonator for the measurement of the relative permittivities of gases, J. Chem. Thermodyn. 34 (2002) 1073-1088. doi:10.1006/jcht.2002.0962.

[56] B. Kapilevich, S.G. Ogourtsov, V.G. Belenky, A.B. Maslenikov, A.S. Omar, Accurate microwave resonant method for complex permittivity measurements of liquids, IEEE Trans. Microw. Theory Tech. 48 (2000) 21592164. doi:10.1109/22.884209.

[57] B. Kapilevich, B. Litvak, Optimized microwave sensor for online concentration measurements of binary liquid mixtures, IEEE Sens. J. 11 (2011) 2611-2616. doi:10.1109/JSEN.2011.2149517.

[58] H. Kawabata, H. Tanpo, R. Members, An improvement of the perturbation method using a TM 010 mode cylindrical cavity, IEICE Trans. Electron. E86 (2003) 2371-2378.

[59] A.J. Estin, E. Bussey, Errors in dielectric measurements due to a sample insertion hole in a Cavity, IRE Trans. Microw. Theory Tech. 8 (1960) 650653.

[60] H. Kawabata, H. Tanpo, Y. Kobayashi, A rigorous analysis of a TM010 mode 
cylindrical cavity to measure accurate complex permittivity of liquid, in: 33rd Eur. Microw. Conf. 2003, 2003. doi:10.1109/EUMA.2003.341064.

[61] H. Kawabata, Y. Kobayashi, Accurate measurements of complex permittivity of liquid based on a $\mathrm{TM}_{010}$ mode cylindrical cavity method, 2005 Eur. Microw. Conf. 1 (2005) 369-372.

[62] P. Kanpan, E. Khansalee, N. Puangngernmak, S. Chalermwisutkul, TM010 mode cylindrical cavity for complex permittivity measurement of liquid using field analysis technique, in: 2012 9th Int. Conf. Electr. Eng. Comput. Telecommun. Inf. Technol., 2012: pp. 1-4. doi:10.1109/ECTICon.2012.6254274.

[63] D.M. Pozar, Microwave Engineering four edition, John Wiley \& Sons, 2012. doi:10.1080/01422419908228843.

[64] G. Birnbaum, J. Franeau, Measurement of the dielectric constant and loss of solids and liquids by a cavity perturbation method, J. Appl. Phys. 20 (1949) 817-818. doi:10.1063/1.1698535.

[65] P. Russer, Electromagnetics, Microwave Circuits and Antenna Design for Communications Engineering (Antennas \& Propagation Library), 2014. doi:10.1128/AAC.03728-14.

[66] P.J. Petersan, S.M. Anlage, Measurement of resonant frequency and quality factor of microwave resonators: Comparison of methods, J. Appl. Phys. 84 (1998) 3392-3402. doi:10.1063/1.368498.

[67] K.J. Coakley, J.D. Splett, M.D. Janezic, R.F. Kaiser, Estimation of Q -Factors and Resonant Frequencies, IEEE Trans. Microw. Theory Tech. 51 (2003) 862-868. doi:10.1109/TMTT.2003.808578.

[68] E. Martin, A. Hernandez, M.C. Sanchez, J.M. Zamarro, J. Margineda, Automatic measurement of $Q$ factor and resonant frequency of microwave resonators, J. Phys. E. 14 (1981) 961-962. doi:10.1088/0022$3735 / 14 / 8 / 016$. 
[69] R. Feynman, The Feynman lectures on physics. California Institute of Technology, (2016). http://www.feynmanlectures.caltech.edu/l_49.html.

[70] Peter Barlow, An elementary investigation of the theory of numbers, J.Johnson and Co, 1811.

[71] H. Preston-Thomas, The international temperature scale of 1990 (ITS-90), Metrología. 27 (1990) 3-10. doi:10.1088/0026-1394/27/1/002.

[72] J.H. Kim, M.S. Kim, Vapor-liquid equilibria for the carbon dioxide + propane system over a temperature range from 253.15 to $323.15 \mathrm{~K}$, Fluid Phase Equilib. 239 (2005) 13-19. doi:10.1016/j.fluid.2005.09.006.

[73] B. Saleh, M. Wendland, Measurement of vapor pressures and saturated liquid densities of pure fluids with a new apparatus, J. Chem. Eng. Data. 50 (2005) 429-437. doi:10.1021/je0497496.

[74] L. Gil, S.T. Blanco, C. Rivas, E. Laga, J. Fernández, M. Artal, I. Velasco, Experimental determination of the critical loci for $\left\{n-\mathrm{C}_{6} \mathrm{H}_{14}\right.$ Or $\mathrm{CO}_{2}+$ alkan-1ol\} mixtures. Evaluation of their critical and subcritical behavior using PCSAFT EoS, J. Supercrit. Fluids. 71 (2012) 26-44. doi:10.1016/j.supflu.2012.07.008.

[75] Y. Suehiro, M. Nakajima, K. Yamada, M. Uematsu, Critical parameters of $\left\{\mathrm{xCO}_{2}+(1-\mathrm{x}) \mathrm{CHF}_{3}\right\}$ for $\mathrm{x}=(1.0000,0.7496,0.5013$, and 0.2522$)$, J. Chem. Thermodyn. 28 (1996) 1153-1164.

[76] A. Diefenbacher, M. Crone, M. Turk, Critical properties of $\mathrm{CO}_{2}, \mathrm{CHF}_{3}, \mathrm{SF}_{6}$, $\left(\mathrm{CO}_{2}+\mathrm{CHF}_{3}\right)$, and $\left(\mathrm{CHF}_{3}+\mathrm{SF}_{3}\right)$, J. Chem. Thermodyn. 30 (1998) 481-496.

[77] H. Renon, J.M. Prausnitz, Local compositions in thermodynamic excess functions for liquid mixtures, AIChE J. 14 (1968) 135-144. doi:10.1002/aic.690140124.

[78] J.A. Nelder, R. Mead, A simplex method for function minimization, Comput. J. 7 (1965) 308-313. doi:10.1093/comjnl/7.4.308.

[79] Á. Martín, M.D. Bermejo, F.A. Mato, M.J. Cocero, Teaching advanced 
equations of state in applied thermodynamics courses using open source programs, Educ. Chem. Eng. 6 (2011) e114-e121. doi:10.1016/j.ece.2011.08.003.

[80] H.G. Donnelly, D.L. Katz, Phase equilibria in the carbon dioxide-methane system, Ind. Eng. Chem. 46 (1954) 511-517.

[81] M.S.W. Wei, T.S. Brown, A.J. Kidnay, E.D. Sloan, Vapor + liquid equilibria for the ternary system methane + ethane + carbon dioxide at $230 \mathrm{~K}$ and its constituent binaries at temperatures from 207 to 270 K, J. Chem. Eng. Data. 40 (1995) 726-731. doi:10.1021/je00020a002.

[82] L.A. Webster, A.J. Kidnay, Vapor-liquid equilibria for the methane-propanecarbon dioxide systems at $230 \mathrm{~K}$ and $270 \mathrm{~K}$, J. Chem. Eng. Data. 46 (2001) 759-764. doi:10.1021/je000307d.

[83] T.A. Al-Sahhaf, A.J. Kidnay, E.D. Sloan, Liquid + vapor equilibria in the $\mathrm{N}_{2}+$ $\mathrm{CO}_{2}+\mathrm{CH}_{4}$ system, Ind. Eng. Chem. Fundam. 22 (1983) 372-380.

[84] J. Davalos, W.R. Anderson, R.E. Phelps, A.J. Kidnay, Liquid-vapor equilibria at $250.00 \mathrm{~K}$ for systems containing methane, ethane, and carbon dioxide, J. Chem. Eng. Data. 21 (1976) 81-84. doi:10.1021/je60068a030.

[85] V.G. Niesen, J.C. Rainwater, Critical locus, (vapor + liquid) equilibria, and coexisting densities of (carbon dioxide + propane) at temperatures from $311 \mathrm{~K}$ to $361 \mathrm{~K}$, J. Chem. Thermodyn. 22 (1990) 777-795. doi:10.1016/00219614(90)90070-7.

[86] W.W. Akers, R.E. Kelley, T.G. Lipscomb, Carbon-dioxide propane system, Ind. Eng. Chem. 46 (1954) 2535-2536. doi:10.1021/ie50540a039.

[87] S.E.M. Hamam, B.C.Y. Lu, Isothermal vapor-liquid equilibria in binary system propane-carbon dioxide, J. Chem. Eng. Data. 21 (1976) 200-204. doi:10.1021/je60069a020.

[88] T.S. Brown, V.G. Niesen, E.D. Sloan, A.J. Kidnay, Vapor-liquid equilibria for the binary systems of nitrogen, carbon dioxide, and n-butane at 


\section{Conclusions}

temperatures from 220 to 344 K, Fluid Phase Equilib. 53 (1989) 7-14. doi:10.1016/0378-3812(89)80067-6.

[89] O. Fandiño, J.P.M. Trusler, D. Vega-Maza, Phase behavior of $\left(\mathrm{CO}_{2}+\mathrm{H}_{2}\right)$ and $\left(\mathrm{CO}_{2}+\mathrm{N}_{2}\right)$ at temperatures between (218.15 and 303.15) $\mathrm{K}$ at pressures up to 15MPa, Int. J. Greenh. Gas Control. 36 (2015) 78-92. doi:10.1016/j.ijggc.2015.02.018.

[90] S.F. Westman, H.G.J. Stang, S.W. Løvseth, A. Austegard, I. Snustad, S.T. Størset, I.S. Ertesvåg, Vapor-liquid equilibrium data for the carbon dioxide and nitrogen $\left(\mathrm{CO}_{2}+\mathrm{N}_{2}\right)$ system at the temperatures 223, 270, 298 and 303 $\mathrm{K}$ and pressures up to $18 \mathrm{MPa}$, Fluid Phase Equilib. 409 (2016) 207-241. doi:10.1016/j.fluid.2015.09.034. 